\title{
Pd-Catalyzed Regio- and Enantioselective Aminoarylation of Allenols with Aryl Iodides and 2-Pyridones
}

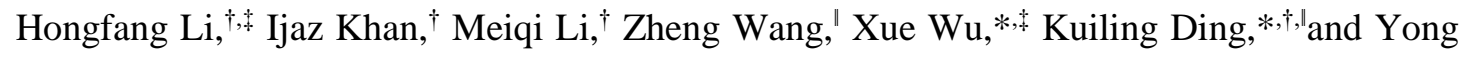
Jian Zhang*,†,

'Frontiers Science Center for Transformative Molecules, Shanghai Key Laboratory for Molecular Engineering of Chiral Drugs, and School of Chemistry and Chemical Engineering, Shanghai Jiao Tong University, 800 Dongchuan Road, Shanghai 200240, P. R. China; "Department of Chemistry, College of Science, Yanbian University, 977 Gongyuan Road, Yanji, Jilin 133002, P. R. China; 'State Key Laboratory of Organometallic Chemistry, Center of Excellence in Molecular Synthesis, Shanghai Institute of Organic Chemistry, Chinese Academy of Sciences, 345 Lingling Road, Shanghai 200032, P. R. China 


\section{Table of Contents}

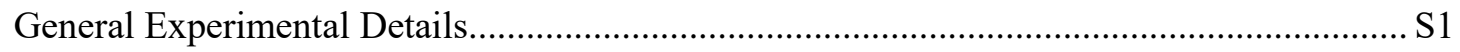

General Procedure and Product Characterization............................................................ S2

General Procedures for the Tandem Aminoarylation of Allenol 1a (conditions A)......... S2

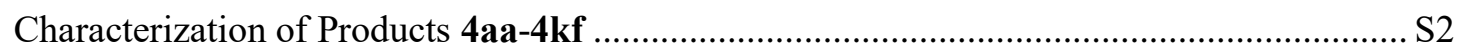

Optimization of Reaction Conditions for 1a with 2a and 5-Nitro-2-pyridone (3f) ....... S15

General Procedures for the Tandem Aminoarylation of Allenol 1a (conditions B)....... S18

General Procedures for the Tandem Aminoarylation of Allenol 1b............................. S18

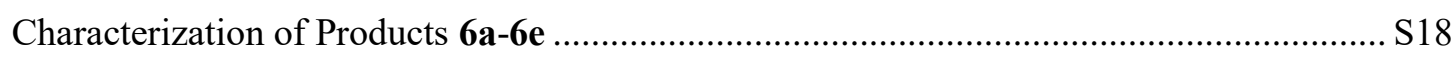

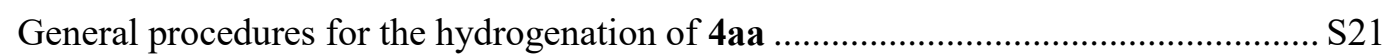

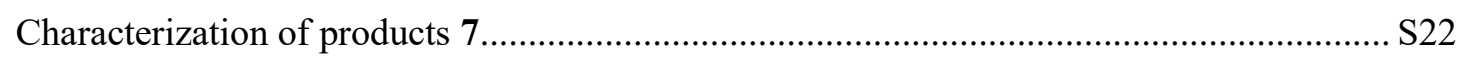

General Procedures for the Synthesis of 2-pyridone compound 10 ........................... S22

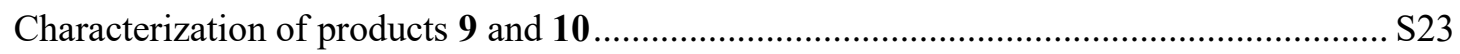

X-Rays Crystallography of 4aa (CCDC2036607) ........................................................... S24

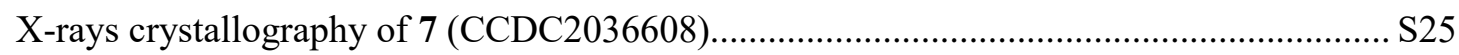

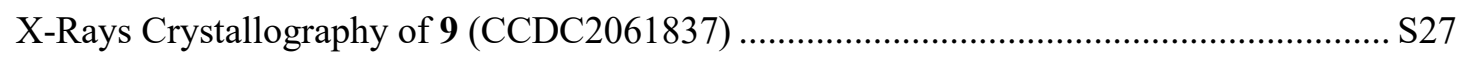

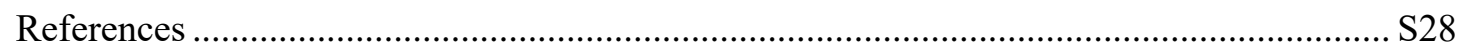

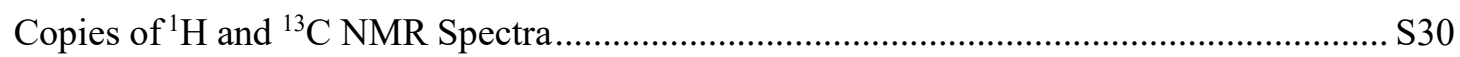

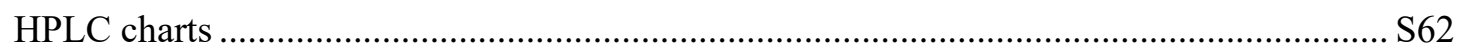




\section{General Experimental Details}

Analytical thin-layer chromatography (TLC) was carried out using 0.2-mm commercial silica gel plates (Yantai Jiangyou Silica Gel Development Co., Ltd., silica gel HSGF 254). Reactions were monitored by thin-layer chromatography (TLC) on silica plates (F-254) and visualized under UV light. Preparative column chromatography employing silica gel (Qingdao Shenghai Fine Silica Gel Chemical Co., Ltd., 200-300 mesh) was performed according to the method of Still. High-resolution mass spectra (HRMS) were performed at Instrumental Analysis Center of Shanghai Jiao Tong University using ESI method. Proton nuclear magnetic resonance $\left({ }^{1} \mathrm{H}\right.$ NMR) spectra were recorded with a Bruker AVANCE III HD 500 (500 MHz) spectrometer. Chemical shifts are reported in delta $(\delta)$ units, parts per million (ppm) downfield from trimethylsilane or ppm relative to the center of the singlet at $7.26 \mathrm{ppm}$ for deuteriochloroform. Coupling constants are reported in Hertz (Hz). Carbon-13 nuclear magnetic resonance $\left({ }^{13} \mathrm{C}\right.$ NMR) spectra were recorded with a Bruker AVANCE III HD 500 (125 MHz) spectrometer. Chemical shifts are reported in delta $(\delta)$ units, ppm relative to the center of the triplet at 77.0 ppm for deuteriochloroform. ${ }^{13} \mathrm{C}$ NMR spectra were routinely run with broadband decoupling. ${ }^{31} \mathrm{P}$ NMR spectra were recorded on Bruker AVANCE III HD 500 (500 MHz) spectrometer. Chemical shifts ( $\delta$ values) were reported in ppm downfield from internal $85 \% \mathrm{H}_{3} \mathrm{PO}_{4}$. Buta2,3-allenol 1a, 3,4-allenol 1b, 4,5-Allenol 1c, 2,3-allenol ether 1d, and alkyl-substituted allene 1e were synthesized according to reported procedures, ${ }^{1-5}$ all characterization data are in accordance with literature. Unless otherwise noted, all reagents and solvents were obtained from commercial sources and used without further purification. 


\section{General Procedure and Product Characterization}

\section{General Procedures for the Tandem Aminoarylation of Allenol 1a (conditions A)}
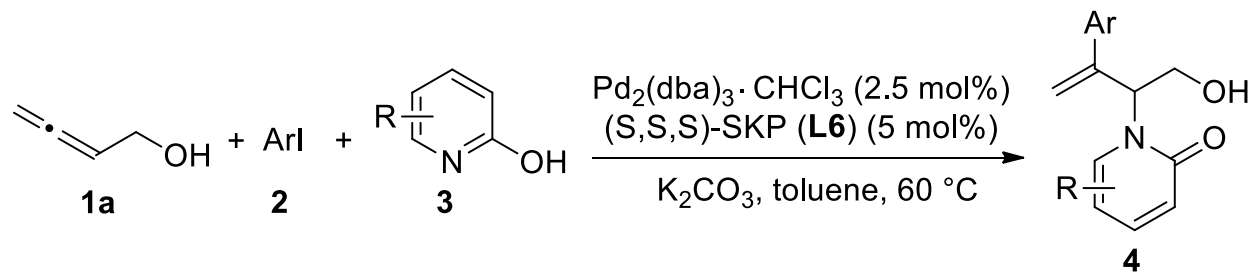

Allenol $1 \mathrm{a}(0.2 \mathrm{mmol}, 1.0$ equiv.), aryl iodides 2 ( $0.24 \mathrm{mmol}, 1.2$ equiv.) and 2-Pyridones 3 (0.24 mmol, 1.2 equiv.) were consecutively added to a sealed tube charged with a mixture of $\mathrm{K}_{2} \mathrm{CO}_{3}$ (27.6 mg, $0.2 \mathrm{mmol}, 1.0$ equiv.), $\mathrm{Pd}_{2}(\mathrm{dba})_{3} \cdot \mathrm{CHCl}_{3}(5.2 \mathrm{mg}, 0.005 \mathrm{mmol}, 2.5 \mathrm{~mol} \%$ ), and $(S, S, S)$-SKP $(6.1 \mathrm{mg}, 0.01 \mathrm{mmol}, 5 \mathrm{~mol} \%)$ under an atmosphere of nitrogen. Toluene $(2 \mathrm{~mL})$ were added sequentially. The reaction mixture was stirred at $60{ }^{\circ} \mathrm{C}$ (oil bath) for $16 \mathrm{~h}$. After cooling to room temperature, the solvent was removed in vacuum. The residue was purified by flash column chromatography on silica gel to afford products 4 .

\section{Characterization of Products 4aa-4kf}

\section{(R)-1-(1-hydroxy-3-phenylbut-3-en-2-yl)pyridin-2(1H)-one (4aa)}

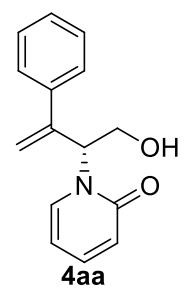

The crude product was purified by flash column chromatography (6:1 of EtOAc:Petroleum ether) on silica gel to provide the title compound; yield: $70 \%(33.8 \mathrm{mg})$; white solid; $127-129^{\circ} \mathrm{C}$; $[\alpha]_{D}^{25}=-141.333\left(c=0.90, \mathrm{CH}_{2} \mathrm{Cl}_{2}\right) ;{ }^{1} \mathrm{H}$ NMR $\left(500 \mathrm{MHz}, \mathrm{CDCl}_{3}\right) \delta 7.49-7.31(\mathrm{~m}, 3 \mathrm{H}), 7.31-$ $7.13(\mathrm{~m}, 4 \mathrm{H}), 6.47(\mathrm{~d}, J=9.1 \mathrm{~Hz}, 1 \mathrm{H}), 6.29(\mathrm{~s}, 1 \mathrm{H}), 6.08(\mathrm{t}, J=6.7 \mathrm{~Hz}, 1 \mathrm{H}), 5.71(\mathrm{~s}, 1 \mathrm{H}), 5.48$ $(\mathrm{d}, J=1.1 \mathrm{~Hz}, 1 \mathrm{H}), 4.29(\mathrm{~s}, 1 \mathrm{H}), 4.19-4.11(\mathrm{~m}, 1 \mathrm{H}), 4.07-3.96(\mathrm{~m}, 1 \mathrm{H}) ;{ }^{13} \mathrm{C}$ NMR $(125 \mathrm{MHz}$, $\left.\mathrm{CDCl}_{3}\right) \delta 163.1,144.5,139.5,138.4,135.0,128.4,128.1,126.1,120.0,117.0,106.3,62.2,57.5$; HRMS (ESI-TOF) m/z: [M + Na $]^{+}$Calcd for $\mathrm{C}_{15} \mathrm{H}_{15} \mathrm{NO}_{2} \mathrm{Na}^{+}$264.1000; Found 264.1002; HPLC 
conditions: Chiralpak AS-H column, $254 \mathrm{~nm}$, flow rate: $1.0 \mathrm{~mL} / \mathrm{min}, i-\mathrm{PrOH} / \mathrm{h}$ exanes $=1 / 3$, $t_{\text {minor }}=13.33$ min, $t_{\text {major }}=16.16 \min ; 95 \%$ ee.

\section{(R)-1-(1-hydroxy-3-(p-tolyl)but-3-en-2-yl)pyridin-2(1H)-one (4ba)}

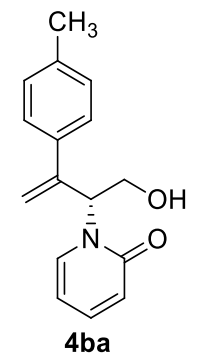

The crude product was purified by flash column chromatography (10:1 of EtOAc:Petroleum ether) on silica gel to provide the title compound; yield: 67\% (34.2 mg); colorless liquid; $[\alpha]_{D}^{25}=-127.391\left(c=0.46, \mathrm{CH}_{2} \mathrm{Cl}_{2}\right) ;{ }^{1} \mathrm{H} \mathrm{NMR}\left(500 \mathrm{MHz}, \mathrm{CDCl}_{3}\right) \delta 7.38(\mathrm{dd}$, $J=6.9,1.9 \mathrm{~Hz}, 1 \mathrm{H}), 7.27(\mathrm{~d}, J=8.2 \mathrm{~Hz}, 2 \mathrm{H}), 7.21(\mathrm{ddd}, J=8.8,6.6,2.0 \mathrm{~Hz}, 1 \mathrm{H}), 7.06(\mathrm{~d}, J=$ $8.0 \mathrm{~Hz}, 2 \mathrm{H}), 6.51(\mathrm{~d}, J=9.1 \mathrm{~Hz}, 1 \mathrm{H}), 6.30(\mathrm{t}, J=5.6 \mathrm{~Hz}, 1 \mathrm{H}), 6.09(\mathrm{td}, J=6.8,1.3 \mathrm{~Hz}, 1 \mathrm{H})$, $5.71(\mathrm{~s}, 1 \mathrm{H}), 5.43(\mathrm{~d}, J=1.5 \mathrm{~Hz}, 1 \mathrm{H}), 4.21-4.17(\mathrm{~m}, 1 \mathrm{H}), 4.06-4.01(\mathrm{~m}, 1 \mathrm{H}), 3.73(\mathrm{~d}, J=5.2$ $\mathrm{Hz}, 1 \mathrm{H}), 2.27$ (s, 3H); ${ }^{13} \mathrm{C}$ NMR $\left(125 \mathrm{MHz}, \mathrm{CDCl}_{3}\right) \delta 163.3,144.2,139.5,138.1,135.4,134.9$, 129.2, 126.0, 120.2, 116.3, 106.4, 62.7, 57.5, 21.0; HRMS (ESI-TOF) m/z: $[\mathrm{M}+\mathrm{Na}]^{+}$Calcd for $\mathrm{C}_{16} \mathrm{H}_{17} \mathrm{NO}_{2} \mathrm{Na}^{+}$278.1158; Found 278.1158; HPLC conditions: Chiralpak AS-H column, 254 $\mathrm{nm}$, flow rate: $1.0 \mathrm{~mL} / \mathrm{min}, i-\mathrm{PrOH} /$ hexanes $=3 / 7$, $\mathrm{t}_{\text {major }}=7.99 \mathrm{~min}, \mathrm{t}_{\text {minor }}=16.05 \mathrm{~min} ; 95 \%$ ee. (R)-1-(1-hydroxy-3-(m-tolyl)but-3-en-2-yl)pyridin-2(1H)-one (4ca)

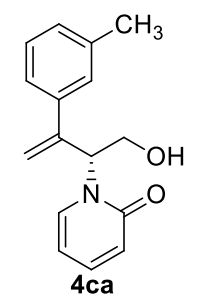

The crude product was purified by flash column chromatography (10:1 of EtOAc:Petroleum ether) on silica gel to provide the title compound; yield: $58 \%$ (29.6 mg); colorless liquid; $[\alpha]_{D}^{25}=-111.111\left(c=0.23, \mathrm{CH}_{2} \mathrm{Cl}_{2}\right) ;{ }^{1} \mathrm{H} \mathrm{NMR}\left(500 \mathrm{MHz}, \mathrm{CDCl}_{3}\right) \delta 7.40(\mathrm{t}$, $J=7.1 \mathrm{~Hz}, 1 \mathrm{H}), 7.25-7.13(\mathrm{~m}, 4 \mathrm{H}), 7.05(\mathrm{~d}, J=6.5 \mathrm{~Hz}, 1 \mathrm{H}), 6.53(\mathrm{t}, J=8.6 \mathrm{~Hz}, 1 \mathrm{H}), 6.31-$ $6.28(\mathrm{~m}, 1 \mathrm{H}), 6.11(\mathrm{t}, J=6.8 \mathrm{~Hz}, 1 \mathrm{H}), 5.71(\mathrm{~d}, J=8.2 \mathrm{~Hz}, 1 \mathrm{H}), 5.42(\mathrm{~d}, J=7.5 \mathrm{~Hz}, 1 \mathrm{H}), 4.22-$ $4.17(\mathrm{~m}, 1 \mathrm{H}), 4.07-4.02(\mathrm{~m}, 1 \mathrm{H}), 3.62(\mathrm{~s}, 1 \mathrm{H}), 2.30(\mathrm{~d}, J=8.4 \mathrm{~Hz}, 3 \mathrm{H}) ;{ }^{13} \mathrm{C}$ NMR $(125 \mathrm{MHz}$, $\left.\mathrm{CDCl}_{3}\right) \delta 163.3,144.6,139.5,138.4,138.1,135.0,129.0,128.4,127.0,123.3,120.2,116.8$, 
106.4, 62.7, 57.7, 21.4; HRMS (ESI-TOF) m/z: $[\mathrm{M}+\mathrm{Na}]^{+}$Calcd for $\mathrm{C}_{16} \mathrm{H}_{17} \mathrm{NO}_{2} \mathrm{Na}^{+}$278.1157; Found 278.1160; HPLC conditions: Chiralpak AS-H column, $254 \mathrm{~nm}$, flow rate: $1.0 \mathrm{~mL} / \mathrm{min}$, $i-\mathrm{PrOH} /$ hexanes $=1 / 3, \mathrm{t}_{\text {minor }}=9.44 \mathrm{~min}, \mathrm{t}_{\text {major }}=13.70 \mathrm{~min} ; 90 \%$ ee.

(R)-1-(1-hydroxy-3-(4-methoxyphenyl)but-3-en-2-yl)pyridin-2(1H)-one (4da)<smiles>C=C(c1ccc(OC)cc1)C(CO)n1ccccc1=O</smiles>

The crude product was purified by flash column chromatography (10:1 of EtOAc:Petroleum ether) on silica gel to provide the title compound; yield: $65 \%$ (35.2 $\mathrm{mg}$ ); colorless liquid; $[\alpha]_{D}^{25}=-42.769\left(c=0.33, \mathrm{CH}_{2} \mathrm{Cl}_{2}\right) ;{ }^{1} \mathrm{H} \mathrm{NMR}\left(500 \mathrm{MHz}, \mathrm{CDCl}_{3}\right) \delta 7.38(\mathrm{dd}$, $J=7.0,1.7 \mathrm{~Hz}, 1 \mathrm{H}), 7.33-7.30(\mathrm{~m}, 2 \mathrm{H}), 7.23-7.19(\mathrm{~m}, 1 \mathrm{H}), 6.79-6.76(\mathrm{~m}, 2 \mathrm{H}), 6.51(\mathrm{~d}, J=$ $9.1 \mathrm{~Hz}, 1 \mathrm{H}), 6.29(\mathrm{t}, J=5.6 \mathrm{~Hz}, 1 \mathrm{H}), 6.09(\mathrm{td}, J=6.8,1.3 \mathrm{~Hz}, 1 \mathrm{H}), 5.66(\mathrm{~s}, 1 \mathrm{H}), 5.40(\mathrm{~d}, J=$ $1.5 \mathrm{~Hz}, 1 \mathrm{H}), 4.18(\mathrm{dd}, J=11.6,4.8 \mathrm{~Hz}, 1 \mathrm{H}), 4.03(\mathrm{dd}, J=11.6,6.4 \mathrm{~Hz}, 1 \mathrm{H}), 3.86(\mathrm{~s}, 1 \mathrm{H}), 3.74$ (s, 3H); ${ }^{13} \mathrm{C} \mathrm{NMR}\left(125 \mathrm{MHz}, \mathrm{CDCl}_{3}\right) \delta 163.3,159.5,143.7,139.5,134.9,130.7,127.3,120.1$, 115.4, 113.8, 106.4, 62.6, 57.4, 55.1; HRMS (ESI-TOF) m/z: $[\mathrm{M}+\mathrm{Na}]^{+}$Calcd for $\mathrm{C}_{16} \mathrm{H}_{17} \mathrm{NO}_{3} \mathrm{Na}^{+}$294.1106; Found 294.1102; HPLC conditions: Chiralpak AS-H column, 254 $\mathrm{nm}$, flow rate: $1.0 \mathrm{~mL} / \mathrm{min}, i-\mathrm{PrOH} /$ hexanes $=1 / 3, \mathrm{t}_{\text {major }}=13.65 \mathrm{~min}, \mathrm{t}_{\operatorname{minor}}=29.42 \mathrm{~min} ; 95 \%$ ee.

\section{(R)-1-(3-(3-fluorophenyl)-1-hydroxybut-3-en-2-yl)pyridin-2(1H)-one (4ea)}

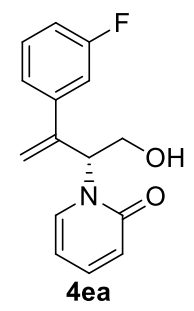

The crude product was purified by flash column chromatography (6:1 of EtOAc:Petroleum ether) on silica gel to provide the title compound; yield: $71 \%(36.8 \mathrm{mg})$; colorless liquid; $[\alpha]_{D}^{25}$ $=-87.000\left(c=0.10, \mathrm{CH}_{2} \mathrm{Cl}_{2}\right) ;{ }^{1} \mathrm{H} \mathrm{NMR}\left(500 \mathrm{MHz}, \mathrm{CDCl}_{3}\right) \delta 7.38(\mathrm{dd}, J=6.9,1.7 \mathrm{~Hz}, 1 \mathrm{H})$, 7.25-7.20 (m, 2H), 7.15 (d, $J=7.8 \mathrm{~Hz}, 1 \mathrm{H}), 7.10(\mathrm{~d}, J=10.2 \mathrm{~Hz}, 1 \mathrm{H}), 6.92(\mathrm{td}, J=8.2,1.5 \mathrm{~Hz}$, $1 \mathrm{H}), 6.51(\mathrm{~d}, J=9.1 \mathrm{~Hz}, 1 \mathrm{H}), 6.25(\mathrm{t}, J=5.1 \mathrm{~Hz}, 1 \mathrm{H}), 6.13(\mathrm{t}, J=6.7 \mathrm{~Hz}, 1 \mathrm{H}), 5.74(\mathrm{~s}, 1 \mathrm{H})$, 
$5.52(\mathrm{~s}, 1 \mathrm{H}), 4.19-4.12(\mathrm{~m}, 1 \mathrm{H}), 4.06-4.02(\mathrm{~m}, 1 \mathrm{H}), 3.95(\mathrm{~s}, 1 \mathrm{H}) ;{ }^{13} \mathrm{C} \mathrm{NMR}\left(125 \mathrm{MHz}, \mathrm{CDCl}_{3}\right)$ $\delta 163.7,163.2,161.8,143.6,140.8,140.8,139.6,134.8,130.10,130.0,121.8,120.2,118.0$, 115.2, 115.0, 113.4, 113.3, 106.5, 62.3, 57.5; HRMS (ESI-TOF) m/z: $[\mathrm{M}+\mathrm{Na}]^{+}$Calcd for $\mathrm{C}_{15} \mathrm{H}_{14} \mathrm{FNO}_{2} \mathrm{Na}^{+}$282.0906; Found 282.0905; HPLC conditions: Chiralpak OD-H column, 254 $\mathrm{nm}$, flow rate: $1.0 \mathrm{~mL} / \mathrm{min}, i-\mathrm{PrOH} / \mathrm{hexanes}=1 / 9, \mathrm{t}_{\text {minor }}=12.56 \mathrm{~min}, \mathrm{t}_{\text {major }}=19.10 \mathrm{~min} ; 88 \%$ ee.

(R)-1-(3-(4-bromophenyl)-1-hydroxybut-3-en-2-yl)pyridin-2(1H)-one (4fa)

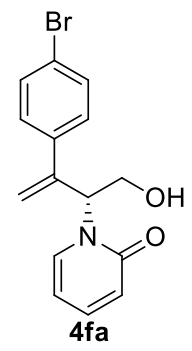

The crude product was purified by flash column chromatography (9:1 of EtOAc:Petroleum ether) on silica gel to provide the title compound; yield: $75 \%$ (47.8 mg); white solid; 140$142{ }^{\circ} \mathrm{C} ;[\alpha]_{D}^{25}=-144.571\left(c=0.35, \mathrm{CH}_{2} \mathrm{Cl}_{2}\right) ;{ }^{1} \mathrm{H}$ NMR $\left(500 \mathrm{MHz}, \mathrm{CDCl}_{3}\right) \delta 7.40-7.37(\mathrm{~m}$, 2H), $7.33(\mathrm{dd}, J=7.0,1.7 \mathrm{~Hz}, 1 \mathrm{H}), 7.27-7.23(\mathrm{~m}, 3 \mathrm{H}), 6.53(\mathrm{~d}, J=8.7 \mathrm{~Hz}, 1 \mathrm{H}), 6.28(\mathrm{dd}, J=$ $6.1,5.0 \mathrm{~Hz}, 1 \mathrm{H}), 6.12(\mathrm{td}, J=6.8,1.3 \mathrm{~Hz}, 1 \mathrm{H}), 5.73(\mathrm{~d}, J=0.8 \mathrm{~Hz}, 1 \mathrm{H}), 5.50(\mathrm{~d}, J=1.7 \mathrm{~Hz}$, $1 \mathrm{H}), 4.21-4.18(\mathrm{~m}, 1 \mathrm{H}), 4.06-4.02(\mathrm{~m}, 1 \mathrm{H}), 3.32(\mathrm{~s}, 1 \mathrm{H}) ;{ }^{13} \mathrm{C} \mathrm{NMR}\left(125 \mathrm{MHz}, \mathrm{CDCl}_{3}\right) \delta 163.2$, 143.6, 139.6, 137.3, 134.6, 131.7, 127.8, 122.4, 120.4, 117.6, 106.6, 62.7, 57.2; HRMS (ESITOF) m/z: [M + Na] ${ }^{+}$Calcd for $\mathrm{C}_{15} \mathrm{H}_{14} \mathrm{BrNO}_{2} \mathrm{Na}^{+}$342.0106; Found 342.0107; HPLC conditions: Chiralpak AS-H column, $254 \mathrm{~nm}$, flow rate: $1.0 \mathrm{~mL} / \mathrm{min}, i-\mathrm{PrOH} /$ hexanes $=1 / 3, \mathrm{t}_{\text {major }}=7.89$ $\min , \mathrm{t}_{\text {minor }}=11.77 \mathrm{~min} ; 94 \%$ ee.

\section{(R)-1-(3-(4-acetylphenyl)-1-hydroxybut-3-en-2-yl)pyridin-2(1H)-one (4ga)}

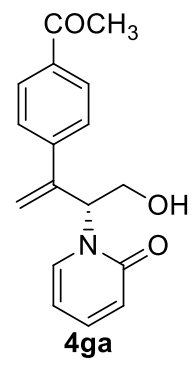

The crude product was purified by flash column chromatography (7:1 of EtOAc:Petroleum ether) on silica gel to provide the title compound; yield: $75 \%$ (42.5 mg); white solid; 154 
$156{ }^{\circ} \mathrm{C} ;[\alpha]_{D}^{25}=-180.444\left(c=0.45, \mathrm{CH}_{2} \mathrm{Cl}_{2}\right) ;{ }^{1} \mathrm{H}$ NMR $\left(500 \mathrm{MHz}, \mathrm{CDCl}_{3}\right) \delta 7.84(\mathrm{~d}, J=8.5$ $\mathrm{Hz}, 2 \mathrm{H}), 7.48$ (d, $J=8.5 \mathrm{~Hz}, 2 \mathrm{H}), 7.36(\mathrm{dd}, J=6.9,1.9 \mathrm{~Hz}, 1 \mathrm{H}), 7.24-7.21(\mathrm{~m}, 1 \mathrm{H}), 6.51(\mathrm{~d}, J$ $=8.7 \mathrm{~Hz}, 1 \mathrm{H}), 6.34(\mathrm{t}, J=5.5 \mathrm{~Hz}, 1 \mathrm{H}), 6.12(\mathrm{td}, J=6.8,1.3 \mathrm{~Hz}, 1 \mathrm{H}), 5.83(\mathrm{~d}, J=0.7 \mathrm{~Hz}, 1 \mathrm{H})$, $5.62(\mathrm{~d}, J=1.7 \mathrm{~Hz}, 1 \mathrm{H}), 4.22-4.18(\mathrm{~m}, 1 \mathrm{H}), 4.09-4.05(\mathrm{~m}, 1 \mathrm{H}), 3.82(\mathrm{t}, J=5.1 \mathrm{~Hz}, 1 \mathrm{H}), 2.54$ (s, 1H); ${ }^{13} \mathrm{C}$ NMR $\left(125 \mathrm{MHz}, \mathrm{CDCl}_{3}\right) \delta$ 197.6, 163.1, 143.9, 143.1, 139.6, 136.6, 134.7, 128.6, 126.4, 120.3, 118.9, 106.6, 62.4, 57.2, 26.5; HRMS (ESI-TOF) $\mathrm{m} / \mathrm{z}:[\mathrm{M}+\mathrm{Na}]^{+}$Calcd for $\mathrm{C}_{17} \mathrm{H}_{17} \mathrm{NO}_{3} \mathrm{Na}^{+}$294.1106; Found 294.1102; HPLC conditions: Chiralpak AS-H column, 254 $\mathrm{nm}$, flow rate: $1.0 \mathrm{~mL} / \mathrm{min}, i-\mathrm{PrOH} / \mathrm{h}$ exanes $=2 / 3, \mathrm{t}_{\text {major }}=11.48 \mathrm{~min}, \mathrm{t}_{\text {minor }}=41.70 \mathrm{~min} ; 95 \%$ ee.

\section{(R)-methyl 4-(4-hydroxy-3-(2-oxopyridin-1(2H)-yl)but-1-en-2-yl)benzoate (4ha)}

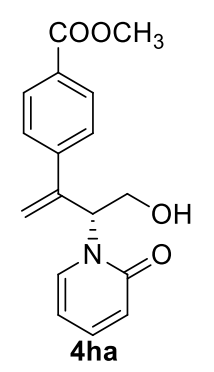

The crude product was purified by flash column chromatography (7:1 of EtOAc:Petroleum ether) on silica gel to provide the title compound; yield: $72 \%(43.1 \mathrm{mg})$; colorless liquid; $[\alpha]_{D}^{25}$ $=-154.615\left(c=0.65, \mathrm{CH}_{2} \mathrm{Cl}_{2}\right) ;{ }^{1} \mathrm{H} \mathrm{NMR}\left(500 \mathrm{MHz}, \mathrm{CDCl}_{3}\right) \delta 7.91(\mathrm{~d}, J=8.3 \mathrm{~Hz}, 2 \mathrm{H}), 7.44(\mathrm{~d}$, $J=8.4 \mathrm{~Hz}, 2 \mathrm{H}), 7.36(\mathrm{dd}, J=7.0,1.8 \mathrm{~Hz}, 1 \mathrm{H}), 7.21(\mathrm{ddd}, J=8.7,6.6,1.9 \mathrm{~Hz}, 1 \mathrm{H}), 6.49(\mathrm{~d}, J$ $=9.1 \mathrm{~Hz}, 1 \mathrm{H}), 6.31(\mathrm{t}, J=5.4 \mathrm{~Hz}, 1 \mathrm{H}), 6.10(\mathrm{td}, J=6.8,1.0 \mathrm{~Hz} 1 \mathrm{H}), 5.81(\mathrm{~s}, 1 \mathrm{H}), 5.60(\mathrm{~d}, J=$ $1.5 \mathrm{~Hz}, 1 \mathrm{H}), 4.23-4.17(\mathrm{~m}, 1 \mathrm{H}), 4.07-4.02(\mathrm{~m}, 1 \mathrm{H}), 3.87(\mathrm{~s}, 3 \mathrm{H}) ;{ }^{13} \mathrm{C} \mathrm{NMR}\left(125 \mathrm{MHz}, \mathrm{CDCl}_{3}\right)$ $\delta$ 166.6, 163.1, 143.9, 143.0, 139.6, 134.8, 129.8, 129.7, 126.2, 120.2, 118.7, 106.5, 62.2, 57.3, 52.0; HRMS (ESI-TOF) m/z: [M + Na $]^{+}$Calcd for $\mathrm{C}_{16} \mathrm{H}_{17} \mathrm{NO}_{4} \mathrm{Na}^{+}$322.1055; Found 322.1053; HPLC conditions: Chiralpak AS-H column, $254 \mathrm{~nm}$, flow rate: $1.0 \mathrm{~mL} / \mathrm{min}, i$-PrOH/hexanes = $1 / 3, t_{\text {major }}=15.56 \mathrm{~min}, t_{\text {minor }}=23.75 \mathrm{~min} ; 95 \%$ ee.

(R)-4-(4-hydroxy-3-(2-oxopyridin-1(2H)-yl)but-1-en-2-yl)benzonitrile (4ia) 


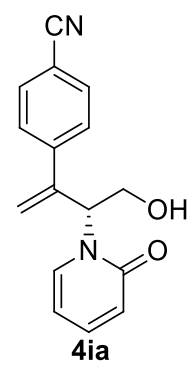

The crude product was purified by flash column chromatography (8:1 of EtOAc:Petroleum ether) on silica gel to provide the title compound; yield: $65 \%$ (34.6 mg); colorless liquid; $[\alpha]_{D}^{25}$ $=-199.176\left(c=0.85, \mathrm{CH}_{2} \mathrm{Cl}_{2}\right) ;{ }^{1} \mathrm{H} \mathrm{NMR}\left(500 \mathrm{MHz}, \mathrm{CDCl}_{3}\right) \delta 7.55(\mathrm{~d}, J=8.5 \mathrm{~Hz}, 2 \mathrm{H}), 7.49(\mathrm{~d}$, $J=8.5 \mathrm{~Hz}, 2 \mathrm{H}), 7.34(\mathrm{dd}, J=6.9,1.5 \mathrm{~Hz}, 1 \mathrm{H}), 7.25(\mathrm{ddd}, J=8.8,6.6,1.9 \mathrm{~Hz}, 1 \mathrm{H}), 6.51(\mathrm{~d}, J$ $=9.1 \mathrm{~Hz}, 1 \mathrm{H}), 6.29(\mathrm{t}, J=5.3 \mathrm{~Hz}, 1 \mathrm{H}), 6.14(\mathrm{td}, J=6.8,0.8 \mathrm{~Hz}, 1 \mathrm{H}), 5.82(\mathrm{~s}, 1 \mathrm{H}), 5.66(\mathrm{~d}, J=$ $1.3 \mathrm{~Hz}, 1 \mathrm{H}), 4.19-4.16(\mathrm{~m}, 1 \mathrm{H}), 4.08-4.04(\mathrm{~m}, 1 \mathrm{H}), 3.92(\mathrm{~s}, 1 \mathrm{H}) ;{ }^{13} \mathrm{C} \mathrm{NMR}\left(125 \mathrm{MHz}, \mathrm{CDCl}_{3}\right)$ $\delta$ 163.0, 143.4, 143.0, 139.8, 134.6, 132.4, 126.9, 120.3, 119.7, 118.4, 111.8, 106.7, 62.1, 57.0; HRMS (ESI-TOF) m/z: $[\mathrm{M}+\mathrm{Na}]^{+}$Calcd for $\mathrm{C}_{16} \mathrm{H}_{14} \mathrm{~N}_{2} \mathrm{O}_{2} \mathrm{Na}^{+}$289.0953; Found 289.0955; HPLC conditions: Chiralpak AS-H column, $254 \mathrm{~nm}$, flow rate: $1.0 \mathrm{~mL} / \mathrm{min}, i-\mathrm{PrOH} /$ hexanes $=$ $1 / 3, \mathrm{t}_{\text {major }}=15.51 \mathrm{~min}, \mathrm{t}_{\mathrm{minor}}=29.76 \mathrm{~min} ; 95 \%$ ee.

\section{(R)-1-(1-hydroxy-3-(4-nitrophenyl)but-3-en-2-yl)pyridin-2(1H)-one (4ja)}

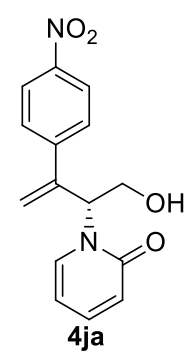

The crude product was purified by flash column chromatography (9:1 of EtOAc:Petroleum ether) on silica gel to provide the title compound; yield: $67 \%$ (38.3 mg); yellow solid; 180 $182{ }^{\circ} \mathrm{C} ;[\alpha]_{D}^{25}=-43.333\left(c=0.15, \mathrm{CH}_{2} \mathrm{Cl}_{2}\right) ;{ }^{1} \mathrm{H} \mathrm{NMR}\left(500 \mathrm{MHz}, \mathrm{CDCl}_{3}\right) \delta 8.13(\mathrm{~d}, J=8.8 \mathrm{~Hz}$, 2H), 7.59-7.54 (m, 2H), 7.32 (dd, $J=6.9,1.9 \mathrm{~Hz}, 1 \mathrm{H}), 7.28-7.22(\mathrm{~m}, 1 \mathrm{H}), 6.54(\mathrm{~d}, J=9.1 \mathrm{~Hz}$, $1 \mathrm{H}), 6.36(\mathrm{t}, J=5.4 \mathrm{~Hz}, 1 \mathrm{H}), 6.13(\mathrm{td}, J=6.8,1.3 \mathrm{~Hz}, 1 \mathrm{H}), 5.88(\mathrm{~s}, 1 \mathrm{H}), 5.69(\mathrm{~d}, J=1.5 \mathrm{~Hz}$, 1H), 4.24-4.21 (m, 1H), 4.11-4.08 (m, 1H), $3.03(\mathrm{~s}, 1 \mathrm{H}) ;{ }^{13} \mathrm{C} \mathrm{NMR}\left(125 \mathrm{MHz}, \mathrm{CDCl}_{3}\right) \delta 163.1$, $147.58,144.9,143.2,139.8,134.4,127.2,123.9,120.6,120.2,106.77,62.6,57.0$; HRMS (ESITOF) m/z: $[\mathrm{M}+\mathrm{Na}]^{+}$Calcd for $\mathrm{C}_{16} \mathrm{H}_{14} \mathrm{~N}_{2} \mathrm{O}_{2} \mathrm{Na}^{+}$309.0851; Found 309.0856; HPLC conditions: 
Chiralpak AS-H column, $254 \mathrm{~nm}$, flow rate: $1.0 \mathrm{~mL} / \mathrm{min}, i-\operatorname{PrOH} /$ hexanes $=1 / 3, \mathrm{t}_{\text {major }}=18.47$ $\min , \mathrm{t}_{\text {minor }}=43.74 \mathrm{~min} ; 84 \%$ ee.

(R)-1-(1-hydroxy-3-(naphthalen-2-yl)but-3-en-2-yl)pyridin-2(1H)-one (4ka)

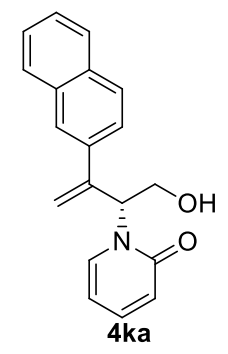

The crude product was purified by flash column chromatography ( $8: 1$ of EtOAc:Petroleum ether) on silica gel to provide the title compound; yield: $76 \%$ (44.2 mg); colorless liquid; $[\alpha]_{D}^{25}$ $=-134.000\left(c=0.20, \mathrm{CH}_{2} \mathrm{Cl}_{2}\right) ;{ }^{1} \mathrm{H}$ NMR $\left(500 \mathrm{MHz}, \mathrm{CDCl}_{3}\right) \delta 7.84(\mathrm{~s}, 1 \mathrm{H}), 7.80-7.74(\mathrm{~m}, 3 \mathrm{H})$, $7.55(\mathrm{dd}, J=8.6,1.5 \mathrm{~Hz}, 1 \mathrm{H}), 7.46-7.42(\mathrm{~m}, 3 \mathrm{H}), 7.20-7.16(\mathrm{~m}, 1 \mathrm{H}), 6.53(\mathrm{~d}, J=9.1 \mathrm{~Hz}, 1 \mathrm{H})$, $6.49(\mathrm{t}, J=5.4 \mathrm{~Hz}, 1 \mathrm{H}), 6.07(\mathrm{t}, J=6.7 \mathrm{~Hz}, 1 \mathrm{H}), 5.88(\mathrm{~s}, 1 \mathrm{H}), 5.55(\mathrm{~s}, 1 \mathrm{H}), 4.29-4.25(\mathrm{~m}, 1 \mathrm{H})$, 4.14-3.09 (m, 1H), 3.28-3.26 (m, 1H); $\left.{ }^{13} \mathrm{C} \mathrm{NMR} \mathrm{(125} \mathrm{MHz,} \mathrm{CDCl}_{3}\right) \delta 163.4,144.4,139.6$, 135.6, 134.8, 133.2, 133.1, 128.4, 128.3, 127.4, 126.3, 125.4, 124.1, 120.3, 117.6, 106.5, 63.0, 57.5; HRMS (ESI-TOF) m/z: [M + Na] ${ }^{+}$Calcd for $\mathrm{C}_{16} \mathrm{H}_{17} \mathrm{NO}_{4} \mathrm{Na}^{+}$314.1157; Found 314.1148; HPLC conditions: Chiralpak AS-H column, $254 \mathrm{~nm}$, flow rate: $1.0 \mathrm{~mL} / \mathrm{min}, i-\mathrm{PrOH} /$ hexanes $=$ $1 / 4, t_{\text {minor }}=21.11 \mathrm{~min}, \mathrm{t}_{\text {major }}=23.14 \mathrm{~min} ; 97 \%$ ee.

(R)-1-(1-hydroxy-3-(naphthalen-1-yl)but-3-en-2-yl)pyridin-2(1H)-one (4la)

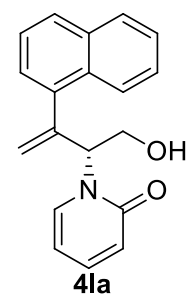

The crude product was purified by flash column chromatography (9:1 of EtOAc:Petroleum ether) on silica gel to provide the title compound; yield: $35 \%$ (20.4 mg); colorless liquid; $[\alpha]_{D}^{25}$ $=-5.946\left(c=0.37, \mathrm{CH}_{2} \mathrm{Cl}_{2}\right) ;{ }^{1} \mathrm{H} \mathrm{NMR}\left(500 \mathrm{MHz}, \mathrm{CDCl}_{3}\right) \delta 8.19(\mathrm{~d}, J=8.1 \mathrm{~Hz}, 1 \mathrm{H}), 7.83(\mathrm{~d}, J$ $=8.2 \mathrm{~Hz}, 1 \mathrm{H}), 7.77(\mathrm{~d}, J=8.0 \mathrm{~Hz}, 1 \mathrm{H}), 7.58(\mathrm{dd}, J=6.9,1.2 \mathrm{~Hz}, 1 \mathrm{H}), 7.51-7.44(\mathrm{~m}, 2 \mathrm{H}), 7.40$ $7.32(\mathrm{~m}, 2 \mathrm{H}), 7.28-7.24(\mathrm{~m}, 1 \mathrm{H}), 6.52(\mathrm{~d}, J=9.1 \mathrm{~Hz}, 1 \mathrm{H}), 6.17-6.10(\mathrm{~m}, 2 \mathrm{H}), 5.46(\mathrm{~s}, 1 \mathrm{H})$, $5.42(\mathrm{~s}, 1 \mathrm{H}), 4.07-3.96(\mathrm{~m}, 2 \mathrm{H}), 3.11(\mathrm{~s}, 1 \mathrm{H}) ;{ }^{13} \mathrm{C} \mathrm{NMR}\left(125 \mathrm{MHz}, \mathrm{CDCl}_{3}\right) \delta$ 163.2, 143.6, 139.4, 137.3, 135.6, 133.7, 131.3, 128.4, 128.2, 126.4, 125.9, 125.6, 125.2, 125.1, 120.6, 119.3, 
106.1, 62.3, 61.5; HRMS (ESI-TOF) m/z: [M + Na] ${ }^{+}$Calcd for $\mathrm{C}_{16} \mathrm{H}_{17} \mathrm{NO}_{4} \mathrm{Na}^{+} 314.1157$; Found 314.1158; HPLC conditions: Chiralpak AS-H column, $254 \mathrm{~nm}$, flow rate: $1.0 \mathrm{~mL} / \mathrm{min}, i$ $\mathrm{PrOH} /$ hexanes $=1 / 4, \mathrm{t}_{\text {major }}=7.36 \mathrm{~min}, \mathrm{t}_{\text {minor }}=13.01 \mathrm{~min} ; 13 \%$ ee.

(R)-1-(1-hydroxy-3-phenylbut-3-en-2-yl)-5-methylpyridin-2(1H)-one (4ab)<smiles></smiles>

The crude product was purified by flash column chromatography (2:1 of EtOAc:Petroleum ether) on silica gel to provide the title compound; Conditions A yield: $73 \%$ (37.2 mg); Conditions B yield: 69\% (35.2 mg); colorless liquid; $[\alpha]_{D}^{25}=-85.667\left(c=0.15, \mathrm{CH}_{2} \mathrm{Cl}_{2}\right) ;{ }^{1} \mathrm{H}$ NMR (500 MHz, $\left.\mathrm{CDCl}_{3}\right) \delta 7.39-7.37$ (m, 2H), 7.27-7.20 (m, 4H), 7.13 (s, 1H), 7.07 (dd, $J=$ 9.2, $2.4 \mathrm{~Hz}, 1 \mathrm{H}), 6.46(\mathrm{~d}, J=9.2 \mathrm{~Hz}, 1 \mathrm{H}), 6.30(\mathrm{t}, J=5.6 \mathrm{~Hz}, 1 \mathrm{H}), 5.72(\mathrm{~s}, 1 \mathrm{H}), 5.45(\mathrm{~d}, J=1.5$ $\mathrm{Hz}, 1 \mathrm{H}), 4.19-4.14(\mathrm{~m}, 1 \mathrm{H}), 4.03-4.00(\mathrm{~m}, 2 \mathrm{H}), 1.97$ (s, 3H); ${ }^{13} \mathrm{C} \mathrm{NMR}\left(125 \mathrm{MHz}, \mathrm{CDCl}_{3}\right) \delta$ $162.5,144.6,142.1,138.5,132.1,128.5,128.1,126.1,119.7,116.8,115.5,62.7,57.4,17.2$; HRMS (ESI-TOF) m/z: [M + Na $]^{+}$Calcd for $\mathrm{C}_{16} \mathrm{H}_{17} \mathrm{NO}_{2} \mathrm{Na}^{+}$278.1151; Found 278.1148; HPLC conditions: Chiralpak AD-H column, $254 \mathrm{~nm}$, flow rate: $1.0 \mathrm{~mL} / \mathrm{min}, i$-PrOH/hexanes = 1/9, $\mathrm{t}_{\text {minor }}=12.44 \mathrm{~min}, \mathrm{t}_{\text {major }}=14.82 \mathrm{~min} ; 97 \%$ ee; $\mathrm{t}_{\text {minor }}=12.83 \mathrm{~min}, \mathrm{t}_{\text {major }}=15.15 \mathrm{~min} ; 81 \%$ ee.

(R)-1-(1-hydroxy-3-phenylbut-3-en-2-yl)-4-methylpyridin-2(1H)-one (4ac)<smiles>C=C(c1ccccc1)[C@H](CO)n1ccc(C(C)(C)C)cc1=O</smiles>

The crude product was purified by flash column chromatography (2:1 of EtOAc:Petroleum ether) on silica gel to provide the title compound; Conditions A yield: 58\% (29.6 mg); Conditions B yield: $65 \%$ (33.2 mg); colorless liquid; $[\alpha]_{D}^{25}=-49.697\left(c=0.16, \mathrm{CH}_{2} \mathrm{Cl}_{2}\right) ;{ }^{1} \mathrm{H}$ NMR (500 MHz, $\left.\mathrm{CDCl}_{3}\right) \delta 7.38(\mathrm{~d}, J=6.8 \mathrm{~Hz}, 2 \mathrm{H}), 7.27-7.20(\mathrm{~m}, 4 \mathrm{H}), 6.30(\mathrm{~s}, 1 \mathrm{H}), 6.26(\mathrm{t}, J$ $=5.1 \mathrm{~Hz}, 1 \mathrm{H}), 5.94(\mathrm{~d}, J=7.0 \mathrm{~Hz}, 1 \mathrm{H}), 5.71(\mathrm{~s}, 1 \mathrm{H}), 5.47(\mathrm{~s}, 1 \mathrm{H}), 4.25(\mathrm{~s}, 1 \mathrm{H}), 4.17-4.12(\mathrm{~m}$, 1H), $4.01(\mathrm{~s}, 1 \mathrm{H}), 2.05(\mathrm{~s}, 3 \mathrm{H}) ;{ }^{13} \mathrm{C}$ NMR (125 MHz, $\left.\mathrm{CDCl}_{3}\right) \delta 163.1,151.2,144.6,138.5$, 133.79, 128.5, 128.1, 126.1, 118.5, 116.9, 109.0, 62.5, 57.2, 21.0; HRMS (ESI-TOF) m/z: [M 
$+\mathrm{Na}]^{+}$Calcd for $\mathrm{C}_{16} \mathrm{H}_{17} \mathrm{NO}_{2} \mathrm{Na}^{+} 278.1151$; Found 278.1151; HPLC conditions: Chiralpak OJ$\mathrm{H}$ column, $254 \mathrm{~nm}$, flow rate: $1.0 \mathrm{~mL} / \mathrm{min}, i-\mathrm{PrOH} /$ hexanes $=1 / 19, \mathrm{t}_{\text {major }}=30.75 \mathrm{~min}, \mathrm{t}_{\mathrm{minor}}=$ $34.24 \mathrm{~min} ; 84 \%$ ee; $\mathrm{t}_{\text {major }}=30.87 \mathrm{~min}, \mathrm{t}_{\mathrm{minor}}=34.43 \mathrm{~min} ; 78 \%$ ee.

(R)-1-(1-hydroxy-3-phenylbut-3-en-2-yl)-3-methylpyridin-2(1H)-one (4ad)<smiles></smiles>

The crude product was purified by flash column chromatography (1:1 of EtOAc:Petroleum ether) on silica gel to provide the title compound; Conditions A yield: $48 \%$ (24.5 mg); Conditions B yield: $60 \%$ (30.6 mg); colorless liquid; $[\alpha]_{D}^{25}=-95.818\left(c=0.55, \mathrm{CH}_{2} \mathrm{Cl}_{2}\right) ;{ }^{1} \mathrm{H}$ NMR (500 MHz, $\left.\mathrm{CDCl}_{3}\right) \delta 7.40-7.37$ (m, 2H), 7.30-7.20 (m, 4H), 7.12-7.10 (m, 1H), 6.37 (t, $J=5.4 \mathrm{~Hz}, 1 \mathrm{H}), 6.04(\mathrm{t}, J=6.9 \mathrm{~Hz}, 1 \mathrm{H}), 5.70(\mathrm{~s}, 1 \mathrm{H}), 5.41(\mathrm{~d}, J=1.6 \mathrm{~Hz}, 1 \mathrm{H}), 4.21-4.18(\mathrm{~m}$, $1 \mathrm{H}), 4.06-4.01(\mathrm{~m}, 1 \mathrm{H}), 3.50(\mathrm{~s}, 1 \mathrm{H}), 2.11(\mathrm{~s}, 3 \mathrm{H}) ;{ }^{13} \mathrm{C} \mathrm{NMR}\left(125 \mathrm{MHz}, \mathrm{CDCl}_{3}\right) \delta 163.7,144.8$, 138.6, 137.0, 132.0, 129.2, 128.5, 128.2, 126.2, 116.8, 106.1, 63.0, 58.0, 17.3; HRMS (ESITOF) $\mathrm{m} / \mathrm{z}:[\mathrm{M}+\mathrm{Na}]^{+}$Calcd for $\mathrm{C}_{16} \mathrm{H}_{17} \mathrm{NO}_{2} \mathrm{Na}^{+} 278.1151$; Found 278.1151; HPLC conditions: Chiralpak AD-H column, $254 \mathrm{~nm}$, flow rate: $1.0 \mathrm{~mL} / \mathrm{min}, i-\mathrm{PrOH} /$ hexanes $=1 / 9, \mathrm{t}_{\mathrm{minor}}=10.54$ min, $\mathrm{t}_{\text {major }}=12.66 \mathrm{~min} ; 74 \%$ ee; $\mathrm{t}_{\text {minor }}=11.09 \mathrm{~min}, \mathrm{t}_{\text {major }}=12.97 \mathrm{~min} ; 81 \%$ ee.

\section{(R)-4-chloro-1-(1-hydroxy-3-phenylbut-3-en-2-yl)pyridin-2(1H)-one (4ae)}

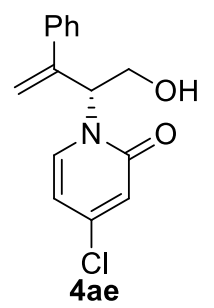

The crude product was purified by flash column chromatography (3:1 of EtOAc:Petroleum ether) on silica gel to provide the title compound; Conditions A yield: $57 \%$ (31.3 mg); Conditions B yield: $60 \%$ (30.0 mg); white solid; $149-151^{\circ} \mathrm{C} ;[\alpha]_{D}^{25}=-99.800(c=0.85$, $\left.\mathrm{CH}_{2} \mathrm{Cl}_{2}\right) ;{ }^{1} \mathrm{H}$ NMR $\left(500 \mathrm{MHz}, \mathrm{CDCl}_{3}\right) \delta$ 7.37-7.33 (m, 3H), 7.30-7.24 (m, 3H), $6.54(\mathrm{~d}, J=2.3$ $\mathrm{Hz}, 1 \mathrm{H}), 6.25-6.22(\mathrm{~m}, 1 \mathrm{H}), 6.12(\mathrm{dd}, J=7.4,2.3 \mathrm{~Hz}, 1 \mathrm{H}), 5.74(\mathrm{~d}, J=1.0 \mathrm{~Hz}, 1 \mathrm{H}), 5.48(\mathrm{~d}, J$ $=1.7 \mathrm{~Hz}, 1 \mathrm{H}), 4.18(\mathrm{dd}, J=11.9,4.6 \mathrm{~Hz}, 1 \mathrm{H}), 4.05(\mathrm{dd}, J=11.9,6.2 \mathrm{~Hz}, 1 \mathrm{H}), 3.29(\mathrm{~s}, 1 \mathrm{H}) ;{ }^{13} \mathrm{C}$ $\operatorname{NMR}\left(125 \mathrm{MHz}, \mathrm{CDCl}_{3}\right) \delta 162.3,146.9,144.2,138.1,135.4,128.7,128.4,126.1,118.7,117.4$, 
108.1, 62.3, 57.4; HRMS (ESI-TOF) m/z: $[\mathrm{M}+\mathrm{Na}]^{+}$Calcd for $\mathrm{C}_{15} \mathrm{H}_{14} \mathrm{ClNO}_{2} \mathrm{Na}^{+}$298.0605; Found 298.0605; HPLC conditions: Chiralpak AD-H column, $254 \mathrm{~nm}$, flow rate: $1.0 \mathrm{~mL} / \mathrm{min}$, $i-\mathrm{PrOH} /$ hexanes $=1 / 9, \mathrm{t}_{\text {major }}=12.47 \mathrm{~min}, \mathrm{t}_{\text {minor }}=14.61 \mathrm{~min} ; 73 \%$ ee; $\mathrm{t}_{\text {major }}=12.41 \mathrm{~min}, \mathrm{t}_{\text {minor }}=$ $14.61 \mathrm{~min} ; 83 \%$ ee.

(R)-1-(1-hydroxy-3-phenylbut-3-en-2-yl)-5-nitropyridin-2(1H)-one (4af)<smiles>C=C(c1ccccc1)C(CO)n1cc([N+](=O)[O-])ccc1=O</smiles>

The crude product was purified by flash column chromatography ( $1: 1$ of EtOAc:Petroleum ether) on silica gel to provide the title compound; Conditions A yield: 62\% (35.5 mg); Conditions B yield: 67\% (38.3 mg); white solid; $112-114{ }^{\circ} \mathrm{C} ;[\alpha]_{D}^{25}=-70.857(c=0.36$, $\left.\mathrm{CH}_{2} \mathrm{Cl}_{2}\right) ;{ }^{1} \mathrm{H} \mathrm{NMR}\left(500 \mathrm{MHz}, \mathrm{CDCl}_{3}\right) \delta 8.67(\mathrm{~d}, J=3.0 \mathrm{~Hz}, 1 \mathrm{H}), 7.97(\mathrm{dd}, J=10.0,3.0 \mathrm{~Hz}$, 1H), 7.37-7.22 (m, 5H), 6.49 (d, $J=10.0 \mathrm{~Hz}, 1 \mathrm{H}), 6.25$ (t, $J=4.6 \mathrm{~Hz}, 1 \mathrm{H}), 5.80$ (s, 1H), 5.59 $(\mathrm{d}, J=1.6 \mathrm{~Hz}, 1 \mathrm{H}), 4.22-4.15(\mathrm{~m}, 2 \mathrm{H}), 2.93(\mathrm{t}, J=4.9 \mathrm{~Hz}, 1 \mathrm{H}) ;{ }^{13} \mathrm{C} \mathrm{NMR}\left(125 \mathrm{MHz}, \mathrm{CDCl}_{3}\right)$ $\delta 162.0,143.3,137.6,133.1,130.9,128.9,128.7,126.1,118.9,118.7,61.6,58.4$; HRMS (ESITOF) m/z: $[\mathrm{M}+\mathrm{Na}]^{+}$Calcd for $\mathrm{C}_{16} \mathrm{H}_{14} \mathrm{~N}_{2} \mathrm{O}_{2} \mathrm{Na}^{+}$309.0852; Found 309.0852; HPLC conditions: Chiralpak AD-H column, $254 \mathrm{~nm}$, flow rate: $1.0 \mathrm{~mL} / \mathrm{min}, i-\mathrm{PrOH} /$ hexanes $=1 / 9, \mathrm{t}_{\text {minor }}=15.86$ min, $\mathrm{t}_{\text {major }}=19.55 \mathrm{~min} ; 78 \%$ ee; $\mathrm{t}_{\text {minor }}=14.33 \mathrm{~min}, \mathrm{t}_{\text {major }}=18.07 \mathrm{~min} ; 86 \%$ ee.

\section{(R)-1-(1-hydroxy-3-phenylbut-3-en-2-yl)-6-oxo-1,6-dihydropyridine-3-carbaldehyde} (4ag)<smiles>C=C(c1ccccc1)C(CO)n1cc(C=O)ccc1=O</smiles>

The crude product was purified by flash column chromatography (2:1 of EtOAc:Petroleum ether) on silica gel to provide the title compound; Conditions A yield: 65\% (35.1 mg); Conditions B yield: 64\% (34.3 mg); white solid; $129-131^{\circ} \mathrm{C} ;[\alpha]_{D}^{25}=-85.158(c=0.95$, $\left.\mathrm{CH}_{2} \mathrm{Cl}_{2}\right) ;{ }^{1} \mathrm{H} \mathrm{NMR}\left(500 \mathrm{MHz}, \mathrm{CDCl}_{3}\right) \delta 9.47(\mathrm{~s}, 1 \mathrm{H}), 8.03(\mathrm{~d}, J=2.4 \mathrm{~Hz}, 1 \mathrm{H}), 7.67(\mathrm{dd}, J=9.5$, $2.4 \mathrm{~Hz}, 1 \mathrm{H}), 7.38-7.34(\mathrm{~m}, 2 \mathrm{H}), 7.29-7.23(\mathrm{~m}, 3 \mathrm{H}), 6.52(\mathrm{~d}, J=9.5 \mathrm{~Hz}, 1 \mathrm{H}), 6.26(\mathrm{t}, J=4.7$ $\mathrm{Hz}, 1 \mathrm{H}), 5.78(\mathrm{~d}, J=0.7 \mathrm{~Hz}, 1 \mathrm{H}), 5.57(\mathrm{~d}, J=1.6 \mathrm{~Hz}, 1 \mathrm{H}), 4.17$ (t, $J=4.9 \mathrm{~Hz}, 2 \mathrm{H}), 3.35$ (t, $J$ 
$=5.5 \mathrm{~Hz}, 1 \mathrm{H}) ;{ }^{13} \mathrm{C} \mathrm{NMR}\left(125 \mathrm{MHz}, \mathrm{CDCl}_{3}\right) \delta 186.4,162.9,145.1,143.8,137.8,135.5,128.8$, 128.6, 126.1, 120.2, 118.1, 118.1, 61.6, 57.9; HRMS (ESI-TOF) m/z: $[\mathrm{M}+\mathrm{Na}]^{+}$Calcd for $\mathrm{C}_{16} \mathrm{H}_{15} \mathrm{NO}_{3} \mathrm{Na}^{+}$292.0944; Found 292.0952; HPLC conditions: Chiralpak OD-H column, 254 $\mathrm{nm}$, flow rate: $1.0 \mathrm{~mL} / \mathrm{min}, i-\mathrm{PrOH} /$ hexanes $=3 / 17, \mathrm{t}_{\text {minor }}=16.11 \mathrm{~min}, \mathrm{t}_{\text {major }}=24.94 \mathrm{~min} ; 83 \%$ ee; $\mathrm{t}_{\text {minor }}=16.76 \mathrm{~min}, \mathrm{t}_{\text {major }}=25.28 \mathrm{~min} ; 89 \%$ ee.

(R)-1-(1-hydroxy-3-phenylbut-3-en-2-yl)-2-oxo-1,2-dihydropyridine-3-carbonitrile (4ah)<smiles>C=C(c1ccccc1)n1cc[14cH]c(C#N)c1=O</smiles>

The crude product was purified by flash column chromatography (4:1 of EtOAc:Petroleum ether) on silica gel to provide the title compound; Conditions A yield: $71 \%$ (37.7 mg); Conditions B yield: $58 \%$ (30.8 mg); colorless liquid; $[\alpha]_{D}^{25}=-53.000\left(c=0.52, \mathrm{CH}_{2} \mathrm{Cl}_{2}\right) ;{ }^{1} \mathrm{H}$ NMR (500 MHz, $\left.\mathrm{CDCl}_{3}\right) \delta 7.80(\mathrm{~s}, 1 \mathrm{H}), 7.39-7.23(\mathrm{~m}, 6 \mathrm{H}), 6.54(\mathrm{~d}, J=9.5 \mathrm{~Hz}, 1 \mathrm{H}), 6.26(\mathrm{t}, J$ $=4.7 \mathrm{~Hz}, 1 \mathrm{H}), 5.74(\mathrm{~s}, 1 \mathrm{H}), 5.51(\mathrm{~s}, 1 \mathrm{H}), 4.20-4.07(\mathrm{~m}, 2 \mathrm{H}), 3.32(\mathrm{t}, J=5.4 \mathrm{~Hz}, 1 \mathrm{H}) ;{ }^{13} \mathrm{C} \mathrm{NMR}$ $\left(125 \mathrm{MHz}, \mathrm{CDCl}_{3}\right) \delta 163.7,144.8,138.6,137.0,132.0,129.2,128.5,128.2,126.2,116.8,106.1$, 63.0, 58.0, 17.3; HRMS (ESI-TOF) m/z: $[\mathrm{M}+\mathrm{Na}]^{+}$Calcd for $\mathrm{C}_{16} \mathrm{H}_{14} \mathrm{~N}_{2} \mathrm{O}_{2} \mathrm{Na}^{+}$289.0947; Found 289.0949; HPLC conditions: Chiralpak AD-H column, $254 \mathrm{~nm}$, flow rate: $1.0 \mathrm{~mL} / \mathrm{min}, i$ $\mathrm{PrOH} /$ hexanes $=3 / 17, \mathrm{t}_{\text {major }}=11.930 \mathrm{~min}, \mathrm{t}_{\text {minor }}=15.948 \mathrm{~min} ; 71 \% \mathrm{ee} ; \mathrm{t}_{\text {major }}=11.365 \mathrm{~min}, \mathrm{t}_{\text {minor }}$ $=14.932 \mathrm{~min} ; 81 \%$ ee.

(R)-3,5-dibromo-1-(1-hydroxy-3-phenylbut-3-en-2-yl)pyridin-2(1H)-one (4ai)<smiles></smiles>

The crude product was purified by flash column chromatography (1:2 of EtOAc:Petroleum ether) on silica gel to provide the title compound; Conditions A yield: 63\% (49.9 mg); Conditions B yield: $61 \%(48.4 \mathrm{mg})$; colorless liquid; $[\alpha]_{D}^{25}=-75.562\left(c=1.6, \mathrm{CH}_{2} \mathrm{Cl}_{2}\right) ;{ }^{1} \mathrm{H}$ NMR (500 MHz, $\left.\mathrm{CDCl}_{3}\right) \delta 7.68(\mathrm{~d}, J=2.5 \mathrm{~Hz}, 1 \mathrm{H}), 7.55(\mathrm{~d}, J=2.5 \mathrm{~Hz}, 1 \mathrm{H}), 7.37-7.25(\mathrm{~m}$, $5 \mathrm{H}), 6.27(\mathrm{t}, J=5.1 \mathrm{~Hz}, 1 \mathrm{H}), 5.73(\mathrm{~s}, 1 \mathrm{H}), 5.49(\mathrm{~d}, J=1.4 \mathrm{~Hz}, 1 \mathrm{H}), 4.21-4.16(\mathrm{~m}, 1 \mathrm{H}), 4.10-$ $4.05(\mathrm{~m}, 1 \mathrm{H}), 3.21(\mathrm{~s}, 1 \mathrm{H}) ;{ }^{13} \mathrm{C} \mathrm{NMR}\left(125 \mathrm{MHz}, \mathrm{CDCl}_{3}\right) \delta 158.3,143.9,143.83,137.9,134.6$, 
128.8, 128.5, 126.1, 117.9, 116.7, 97.3, 62.1, 59.6; HRMS (ESI-TOF) m/z: $[\mathrm{M}+\mathrm{Na}]^{+}$Calcd for $\mathrm{C}_{15} \mathrm{H}_{13} \mathrm{Br}_{2} \mathrm{NO}_{2} \mathrm{Na}^{+}$419.9205; Found 419.9195; HPLC conditions: Chiralpak AD-H column, $254 \mathrm{~nm}$, flow rate: $1.0 \mathrm{~mL} / \mathrm{min}, i-\mathrm{PrOH} /$ hexanes $=1 / 9, \mathrm{t}_{\text {minor }}=9.54 \mathrm{~min}, \mathrm{t}_{\text {major }}=15.33 \mathrm{~min} ; 76 \%$ ee; $\mathrm{t}_{\mathrm{minor}}=9.58 \mathrm{~min}, \mathrm{t}_{\mathrm{major}}=15.41 \mathrm{~min} ; 83 \%$ ee.

\section{(R)-1-(3-(4-bromophenyl)-1-hydroxybut-3-en-2-yl)-5-methylpyridin-2(1H)-one (4fb)}

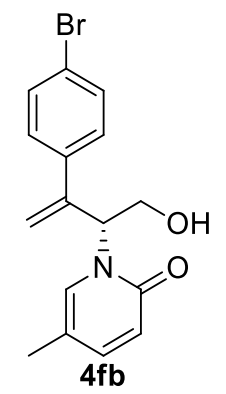

The crude product was purified by flash column chromatography (4:1 of EtOAc:Petroleum ether) on silica gel to provide the title compound; Conditions A yield: $75 \%(50.1 \mathrm{mg})$; Conditions B yield: $73 \%$ (48.6 mg); colorless liquid; $[\alpha]_{D}^{25}=-83.043\left(c=0.23, \mathrm{CH}_{2} \mathrm{Cl}_{2}\right) ;{ }^{1} \mathrm{H}$ $\operatorname{NMR}\left(500 \mathrm{MHz}, \mathrm{CDCl}_{3}\right) \delta$ 7.41-7.37 (m, 2H), 7.27-7.23 (m, 2H), $7.10(\mathrm{dd}, J=9.2,2.5 \mathrm{~Hz}$, 1H), $7.06(\mathrm{~s}, 1 \mathrm{H}), 6.48(\mathrm{~d}, J=9.2 \mathrm{~Hz}, 1 \mathrm{H}), 6.27(\mathrm{t}, J=5.3 \mathrm{~Hz}, 1 \mathrm{H}), 5.73(\mathrm{~d}, J=0.6 \mathrm{~Hz}, 1 \mathrm{H})$, $5.46(\mathrm{~d}, J=1.6 \mathrm{~Hz}, 1 \mathrm{H}), 4.22-4.15(\mathrm{~m}, 1 \mathrm{H}), 4.05-3.99(\mathrm{~m}, 1 \mathrm{H}), 3.28(\mathrm{~s}, 1 \mathrm{H}), 1.99(\mathrm{~s}, 3 \mathrm{H}),{ }^{13} \mathrm{C}$ $\operatorname{NMR}\left(125 \mathrm{MHz}, \mathrm{CDCl}_{3}\right) \delta 162.6,143.6,142.3,137.3,131.7,131.7,127.8,122.4,119.9,117.4$, 115.7, 63.0, 57.1, 17.3; HRMS (ESI-TOF) $\mathrm{m} / \mathrm{z}:[\mathrm{M}+\mathrm{Na}]^{+}$Calcd for $\mathrm{C}_{16} \mathrm{H}_{16} \mathrm{BrNO}_{2} \mathrm{Na}^{+}$ 334.0443; Found 334.0439; HPLC conditions: Chiralpak AD-H column, 254 nm, flow rate: 1.0 $\mathrm{mL} / \mathrm{min}, i-\mathrm{PrOH} / \mathrm{h}_{\text {exanes }}=3 / 17, \mathrm{t}_{\mathrm{minor}}=9.96 \mathrm{~min}, \mathrm{t}_{\mathrm{major}}=12.17 \mathrm{~min} ; 73 \% \mathrm{ee} ; \mathrm{t}_{\mathrm{minor}}=9.77 \mathrm{~min}$, $\mathrm{t}_{\text {major }}=11.90 \mathrm{~min} ; 82 \%$ ee.

(R)-1-(3-(4-bromophenyl)-1-hydroxybut-3-en-2-yl)-5-nitropyridin-2(1H)-one (4ff)<smiles>C=C(c1ccc(Br)cc1)C(CO)n1cc([N+](=O)[O-])ccc1=O</smiles>

The crude product was purified by flash column chromatography (3:1 of EtOAc:Petroleum ether) on silica gel to provide the title compound; Conditions A yield: $68 \%$ (49.5 mg); 
Conditions B yield: $62 \%$ (45.1 mg); colorless liquid; $[\alpha]_{D}^{25}=-23.704\left(c=0.25, \mathrm{CH}_{2} \mathrm{Cl}_{2}\right) ;{ }^{1} \mathrm{H}$ NMR $\left(500 \mathrm{MHz}, \mathrm{CDCl}_{3}\right) \delta 8.64(\mathrm{~d}, J=3.0 \mathrm{~Hz}, 1 \mathrm{H}), 8.00(\mathrm{dd}, J=10.0,3.0 \mathrm{~Hz}, 1 \mathrm{H}), 7.43(\mathrm{~d}, J$ $=8.6 \mathrm{~Hz}, 2 \mathrm{H}), 7.26(\mathrm{~d}, J=4.9 \mathrm{~Hz}, 2 \mathrm{H}), 6.52(\mathrm{~d}, J=10.0 \mathrm{~Hz}, 1 \mathrm{H}), 6.23(\mathrm{t}, J=4.7 \mathrm{~Hz}, 1 \mathrm{H}), 5.81$ (s, 1H), $5.62(\mathrm{~d}, J=1.6 \mathrm{~Hz}, 1 \mathrm{H}), 4.19(\mathrm{~d}, J=4.5 \mathrm{~Hz}, 2 \mathrm{H}), 2.44(\mathrm{~s}, 1 \mathrm{H}) ;{ }^{13} \mathrm{C} \mathrm{NMR}(125 \mathrm{MHz}$, $\left.\mathrm{CDCl}_{3}\right) \delta 161.8,142.4,137.4,136.6,133.2,132.1,130.9,127.8,123.0,119.2,119.1,61.6,58.0$; HRMS (ESI-TOF) m/z: [M + Na $]^{+}$Calcd for $\mathrm{C}_{15} \mathrm{H}_{13} \mathrm{Br}_{2} \mathrm{~N}_{2} \mathrm{O}_{2} \mathrm{Na}^{+}$365.0137; Found 365.0133; HPLC conditions: Chiralpak AD-H column, $254 \mathrm{~nm}$, flow rate: $1.0 \mathrm{~mL} / \mathrm{min}, i$-PrOH/hexanes $=$ $1 / 9, \mathrm{t}_{\text {minor }}=17.49 \mathrm{~min}, \mathrm{t}_{\text {major }}=22.94 \mathrm{~min} ; 87 \%$ ee; $\mathrm{t}_{\text {minor }}=17.11 \mathrm{~min}, \mathrm{t}_{\text {major }}=22.34 \mathrm{~min} ; 93 \%$ ee. (R)-1-(1-hydroxy-3-(naphthalen-2-yl)but-3-en-2-yl)-5-methylpyridin-2(1H)-one (4kb)

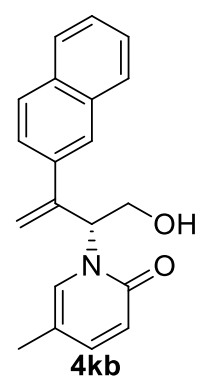

The crude product was purified by flash column chromatography (5:1 of EtOAc:Petroleum ether) on silica gel to provide the title compound; Conditions A yield: 68\% (41.5 mg); Conditions B yield: 68\% (41.3 mg); colorless liquid; $[\alpha]_{D}^{25}=-124.333\left(c=0.3, \mathrm{CH}_{2} \mathrm{Cl}_{2}\right) ;{ }^{1} \mathrm{H}$ NMR (500 MHz, $\left.\mathrm{CDCl}_{3}\right) \delta 7.84(\mathrm{~s}, 1 \mathrm{H}), 7.80-7.73(\mathrm{~m}, 3 \mathrm{H}), 7.55(\mathrm{~d}, J=8.6 \mathrm{~Hz}, 1 \mathrm{H}), 7.46-$ $7.39(\mathrm{~m}, 2 \mathrm{H}), 7.18(\mathrm{~s}, 1 \mathrm{H}), 7.06-7.02(\mathrm{~m}, 2 \mathrm{H}), 6.52-6.44(\mathrm{~m}, 2 \mathrm{H}), 5.88(\mathrm{~s}, 1 \mathrm{H}), 5.54(\mathrm{~s}, 1 \mathrm{H})$, 4.31-4.21 (m, 1H), 4.13-4.05 (m, 1H), $3.54(\mathrm{~s}, 1 \mathrm{H}), 1.95(\mathrm{~s}, 3 \mathrm{H}) ;{ }^{13} \mathrm{C} \mathrm{NMR}\left(125 \mathrm{MHz}, \mathrm{CDCl}_{3}\right)$ $\delta 162.7,144.4,142.2,135.7,133.2,133.0,131.9,128.4,128.2,127.4,126.2,125.3,124.1$, 119.8, 117.3, 115.6, 63.2, 57.4, 17.3; HRMS (ESI-TOF) m/z: $[\mathrm{M}+\mathrm{Na}]^{+}$Calcd for $\mathrm{C}_{20} \mathrm{H}_{19} \mathrm{NO}_{2} \mathrm{Na}^{+}$306.1494; Found 306.1489; HPLC conditions: Chiralpak AD-H column, 254 $\mathrm{nm}$, flow rate: $1.0 \mathrm{~mL} / \mathrm{min}, i-\mathrm{PrOH} /$ hexanes $=2 / 8, \mathrm{t}_{\text {minor }}=7.99 \mathrm{~min}, \mathrm{t}_{\text {major }}=9.50 \mathrm{~min} ; 81 \%$ ee; $\mathrm{t}_{\text {minor }}=8.21 \mathrm{~min}, \mathrm{t}_{\text {major }}=9.81 \mathrm{~min} ; 83 \%$ ee.

(R)-1-(1-hydroxy-3-(naphthalen-2-yl)but-3-en-2-yl)-5-nitropyridin-2(1H)-one (4kf) 


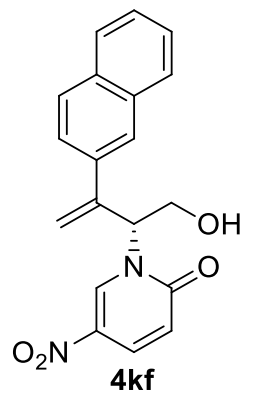

The crude product was purified by flash column chromatography (4:1 of EtOAc:Petroleum ether) on silica gel to provide the title compound; Conditions A yield: $63 \%$ (42.3 mg); Conditions B yield: $65 \%$ (43.7 mg); yellow solid; $166-168{ }^{\circ} \mathrm{C} ;[\alpha]_{D}^{25}=-27.391(c=0.9$, $\left.\mathrm{CH}_{2} \mathrm{Cl}_{2}\right) ;{ }^{1} \mathrm{H} \mathrm{NMR}\left(500 \mathrm{MHz}, \mathrm{CDCl}_{3}\right) \delta 8.73(\mathrm{~d}, J=3.0 \mathrm{~Hz}, 1 \mathrm{H}), 7.93(\mathrm{dd}, J=10.0,3.0 \mathrm{~Hz}$, 1H), 7.82-7.76 (m, 4H), $7.51(\mathrm{dd}, J=8.6,1.7 \mathrm{~Hz}, 1 \mathrm{H}), 7.47-7.44(\mathrm{~m}, 2 \mathrm{H}), 6.48(\mathrm{~d}, J=10.0$ $\mathrm{Hz}, 1 \mathrm{H}), 6.42$ (t, $J=4.7 \mathrm{~Hz}, 1 \mathrm{H}), 5.94(\mathrm{~s}, 1 \mathrm{H}), 5.67$ (d, $J=1.2 \mathrm{~Hz}, 1 \mathrm{H}), 4.29-4.18(\mathrm{~m}, 1 \mathrm{H})$, $2.57(\mathrm{~s}, 1 \mathrm{H}) ;{ }^{13} \mathrm{C} \mathrm{NMR}\left(125 \mathrm{MHz}, \mathrm{CDCl}_{3}\right) \delta 162.0,143.3,137.6,134.9,133.2,133.2,130.9$, 128.7, 128.3, 127.5, 126.6, 126.6, 125.5, 123.9, 119.1, 118.9, 61.9, 58.4; HRMS (ESI-TOF) m/z: $[\mathrm{M}+\mathrm{Na}]^{+}$Calcd for $\mathrm{C}_{19} \mathrm{H}_{17} \mathrm{~N}_{2} \mathrm{O}_{2}{ }^{+}$337.1188; Found 337.1187; HPLC conditions: Chiralpak AD-H column, $254 \mathrm{~nm}$, flow rate: $1.0 \mathrm{~mL} / \mathrm{min}, i-\mathrm{PrOH} /$ hexanes $=3 / 17, \mathrm{t}_{\text {minor }}=11.81$ $\min , \mathrm{t}_{\text {major }}=15.15 \mathrm{~min} ; 87 \%$ ee; $\mathrm{t}_{\text {minor }}=12.35 \mathrm{~min}, \mathrm{t}_{\text {major }}=15.82 \mathrm{~min} ; 93 \%$ ee.

\section{Optimization of Reaction Conditions for 1a with 2a and 5-Nitro-2-pyridone (3f)}

Table S1 Optimizations of Conditions of the Reaction of $1 \mathbf{a}$ with $\mathbf{2 a}$ and $\mathbf{3} \mathbf{f}^{\mathrm{a}}$

\begin{tabular}{|c|c|c|c|c|c|c|}
\hline entry & $\mathrm{T}\left({ }^{\circ} \mathrm{C}\right)$ & base (1eq.) & solvent & yield $(\%)^{\mathrm{b}}$ & e.r..$^{c}$ & 4af:5af ${ }^{\mathrm{d}}$ \\
\hline 1 & 60 & $\mathrm{~K}_{2} \mathrm{CO}_{3}$ & toluene & 65 & $89 / 11$ & $76: 24$ \\
\hline 2 & 60 & $\mathrm{~K}_{2} \mathrm{CO}_{3}$ & THF & 70 & $77 / 23$ & 78:22 \\
\hline 3 & 60 & $\mathrm{~K}_{2} \mathrm{CO}_{3}$ & MTBE & 68 & $59 / 41$ & $75: 25$ \\
\hline 4 & 60 & $\mathrm{~K}_{2} \mathrm{CO}_{3}$ & CPME & 67 & $82 / 18$ & $75: 25$ \\
\hline
\end{tabular}




\begin{tabular}{|c|c|c|c|c|c|c|}
\hline 5 & 60 & $\mathrm{~K}_{2} \mathrm{CO}_{3}$ & $\mathrm{CH}_{3} \mathrm{CN}$ & 71 & $54 / 46$ & 79:21 \\
\hline 6 & 60 & $\mathrm{~K}_{2} \mathrm{CO}_{3}$ & DCE & 73 & $58 / 42$ & $80: 20$ \\
\hline 7 & 60 & $\mathrm{~K}_{2} \mathrm{CO}_{3}$ & dioxane & 65 & $87 / 13$ & $75: 25$ \\
\hline 8 & 40 & $\mathrm{~K}_{2} \mathrm{CO}_{3}$ & toluene & 52 & $81 / 19$ & $67: 33$ \\
\hline 9 & 40 & $\mathrm{~K}_{2} \mathrm{CO}_{3}$ & THF & 62 & $90 / 10$ & $71: 29$ \\
\hline 10 & 40 & $\mathrm{~K}_{2} \mathrm{CO}_{3}$ & MTBE & 68 & $91 / 9$ & $75: 25$ \\
\hline 11 & 40 & $\mathrm{~K}_{2} \mathrm{CO}_{3}$ & CPME & 65 & $87 / 13$ & $74: 26$ \\
\hline 12 & 40 & $\mathrm{~K}_{2} \mathrm{CO}_{3}$ & $\mathrm{CH}_{3} \mathrm{CN}$ & 45 & $63 / 37$ & $67: 33$ \\
\hline 13 & 40 & $\mathrm{~K}_{2} \mathrm{CO}_{3}$ & DCE & 65 & $63 / 37$ & $75: 25$ \\
\hline 14 & 40 & $\mathrm{~K}_{2} \mathrm{CO}_{3}$ & dioxane & 63 & $89 / 11$ & $74: 26$ \\
\hline 15 & 30 & $\mathrm{~K}_{2} \mathrm{CO}_{3}$ & THF & 55 & $90 / 10$ & $67: 33$ \\
\hline 16 & 30 & $\mathrm{~K}_{2} \mathrm{CO}_{3}$ & MTBE & 65 & $93 / 7$ & $74: 26$ \\
\hline 17 & 30 & $\mathrm{~K}_{2} \mathrm{CO}_{3}$ & dioxane & 62 & $93 / 7$ & $71: 29$ \\
\hline 18 & 30 & $\mathrm{~K}_{2} \mathrm{CO}_{3}$ & Ether & 15 & $93 / 7$ & $44: 56$ \\
\hline 19 & 30 & $\mathrm{~K}_{2} \mathrm{CO}_{3}$ & CPME & 60 & $88 / 12$ & $71: 29$ \\
\hline 20 & 20 & $\mathrm{~K}_{2} \mathrm{CO}_{3}$ & MTBE & 54 & $94 / 6$ & $67: 33$ \\
\hline 21 & 20 & $\mathrm{~K}_{2} \mathrm{CO}_{3}$ & dioxane & 50 & $93 / 7$ & $60: 40$ \\
\hline 22 & 30 & $\mathrm{~K}_{3} \mathrm{PO}_{4}$ & MTBE & 67 & $93 / 7$ & $74: 26$ \\
\hline
\end{tabular}

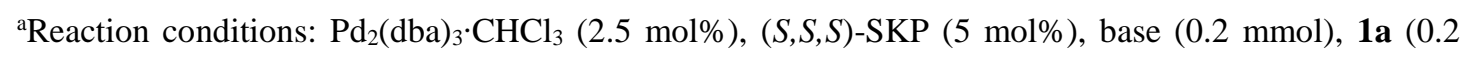
mmol), $2 \mathbf{a}(0.24 \mathrm{mmol}), \mathbf{3 f}(0.24 \mathrm{mmol})$, solvent $(2.0 \mathrm{~mL})$. ${ }^{b}$ Isolated yield for 4af. ${ }^{~}$ Determined by HPLC using chiral stationary phase. ${ }^{\mathrm{d}}$ Determined by ${ }^{1} \mathrm{HNMR}$ spectroscopy of the crude reaction mixture.

Table S2. Pd-Catalyzed Aminoarylation of 2,3-Allenol (1a) with Various Aryl Iodides 2 and 2-pyridones $3^{[a]}$ 


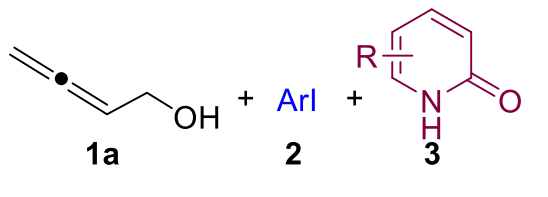

$\mathrm{Pd}_{2}(\mathrm{dba})_{3} \cdot \mathrm{CHCl}_{3}(2.5 \mathrm{~mol} \%)$ (S,S,S)-SKP (L6) (5 mol\%)

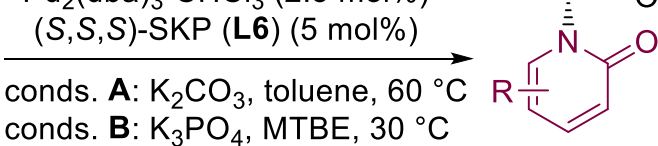

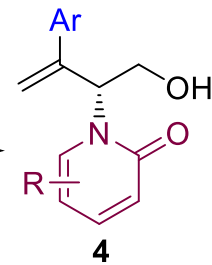<smiles>C=C(c1ccccc1)[C@H](CO)n1cc(C)ccc1=O</smiles>

4ab

A: $73 \%, 98: 2$ er $\mathrm{b}: \mathrm{I}=82: 18$

B: $69 \%, 91: 9 \mathrm{er}$ b: $I=74: 26$<smiles></smiles>

A: $62 \%, 89: 11 \mathrm{er}$ $b: I=76: 24$

B: $67 \%, 93: 7$ er b: $I=74: 26$<smiles>C=C(c1ccc(Br)cc1)[C@H](CO)n1cc(C)ccc1=O</smiles>

$4 \mathrm{fb}$

A: $75 \%, 87: 13$ er $b: I=80: 20$

B: $73 \%, 91: 9$ er $b: I=80: 20$<smiles>C=C(c1ccccc1)[C@H](CO)n1ccc(C)cc1=O</smiles>

$4 a c$

A: $58 \%, 92: 8$ er $b: I=80: 20$

B: $65 \%, 89: 11$ er $b: I=75: 25$<smiles>C=C(c1ccccc1)[C@H](CO)n1cc(C=O)ccc1=O</smiles>

A: $65 \%, 92: 8 \mathrm{er}$ $b: I=69: 31$

B: $64 \%, 95: 5$ er $\mathrm{b}: \mathrm{I}=72: 28$<smiles></smiles>
A: $68 \%, 94: 6$ er $\mathrm{b}: \mathrm{I}=75: 25$
B: $62 \%, 97: 3$ er $b: I=71: 29$<smiles>C=C(c1ccccc1)[C@H](CO)n1cccc(C)c1=O</smiles>

$4 a d$

A: $48 \%, 87: 13$ er b:l = 79:21

B: $60 \%, 91: 9$ er $\mathrm{b}: \mathrm{I}=71: 29$<smiles>C=C(c1ccccc1)[C@H](CO)n1ccc(C#N)c(C#N)c1=O</smiles>

A: $71 \%, 86: 14$ er $\mathrm{b}: \mathrm{I}=78: 22$

B: $58 \%, 91: 9$ er $b: I=67: 33$<smiles>C=C(c1ccc2ccccc2c1)[C@@H](CO)n1cc(C)ccc1=O</smiles>

4kb

A: $68 \%, 91: 9$ er $\mathrm{b}: \mathrm{l}=77: 23$

B: $68 \%, 92: 8 \mathrm{er}$ $\mathrm{b}: \mathrm{l}=76: 24$<smiles>C=C(c1ccccc1)[C@H](CO)n1ccc(Cl)cc1=O</smiles>

4ae

A: $57 \%, 87: 13$ er $b: I=81: 19$

B: $60 \%, 91: 9 \mathrm{er}$ $\mathrm{b}: \mathrm{I}=70: 30$<smiles></smiles>

A: $63 \%, 88: 12 \mathrm{er}$ $b: I=73: 27$

B: $61 \%, 92: 8 \mathrm{er}$ $b: I=71: 29$<smiles>C=C(c1ccc2ccccc2c1)[C@@H](CO)n1cc([N+](=O)[O-])ccc1=O</smiles>
A: $63 \%, 94: 6$ er $b: I=73: 27$
B: $65 \%, 97: 3$ er $b: I=72: 28$

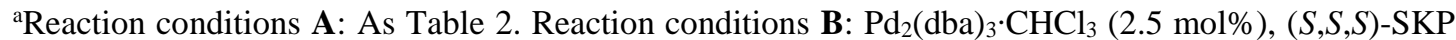
(5 mol\%), $\mathrm{K}_{3} \mathrm{PO}_{4}(0.2 \mathrm{mmol}), \mathbf{1 a}(0.2 \mathrm{mmol}), 2(0.24 \mathrm{mmol}), 3(0.24 \mathrm{mmol}), \mathrm{MTBE}(2.0 \mathrm{~mL}), 30^{\circ} \mathrm{C}(\mathrm{oil}$ bath), $24 \mathrm{~h}$. The yields are of isolated materials. The enantioselectivities were determined by HPLC on a chiral stationary phase. The branched/linear ratios of products were determined by ${ }^{1} \mathrm{H}$ NMR spectroscopy of the crude reaction mixture. MTBE = methyl tert-butyl ether. 


\section{General Procedures for the Tandem Aminoarylation of Allenol 1a (conditions B)}

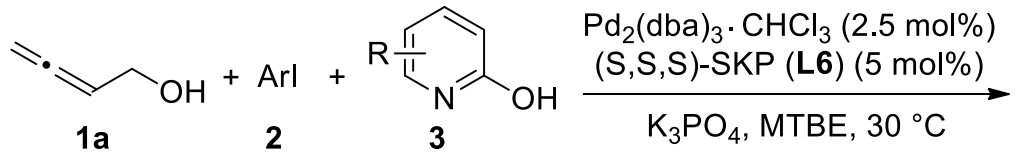

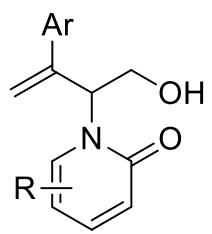

4

2,3-Allenol 1a ( $0.2 \mathrm{mmol}, 1.0$ equiv.), aryl iodides 2 ( $0.24 \mathrm{mmol}, 1.2$ equiv.) and 2Pyridones 3 ( 0.24 mmol, 1.2 equiv.) were consecutively added to a sealed tube charged with a mixture of $\mathrm{K}_{3} \mathrm{PO}_{4}\left(42.4 \mathrm{mg}, 0.2 \mathrm{mmol}, 1.0\right.$ equiv.), $\mathrm{Pd}_{2}\left(\mathrm{dba}_{3} \cdot \mathrm{CHCl}_{3}(5.2 \mathrm{mg}, 0.005 \mathrm{mmol}, 2.5\right.$ mol\%), and (S,S,S)-SKP (6.1 mg, $0.01 \mathrm{mmol}, 5 \mathrm{~mol} \%)$ under an atmosphere of nitrogen. MTBE $\left(2 \mathrm{~mL}\right.$ ) were added sequentially. The reaction mixture was stirred at $30{ }^{\circ} \mathrm{C}$ (oil bath) for $24 \mathrm{~h}$. After cooling to room temperature, the solvent was removed in vacuum. The residue was purified by flash column chromatography on silica gel to afford products 4 .

\section{General Procedures for the Tandem Aminoarylation of Allenol 1b}
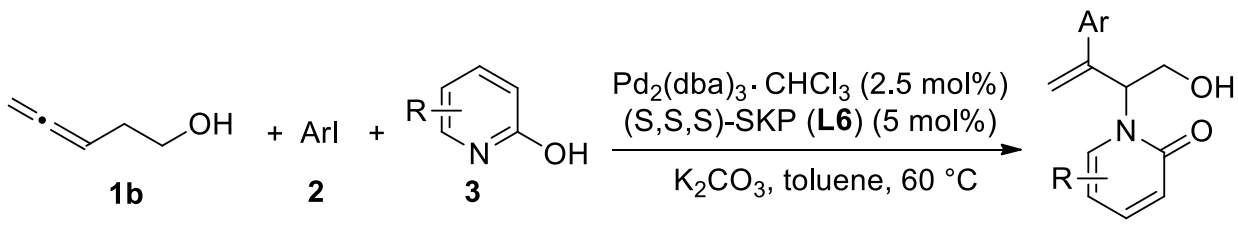

$$
6
$$

Allenol $1 \mathbf{b}$ ( $0.2 \mathrm{mmol}, 1.0$ equiv.), aryl iodides 2 ( $0.24 \mathrm{mmol}, 1.2$ equiv.) and 2-Pyridones 3 (0.24 mmol, 1.2 equiv.) were consecutively added to a sealed tube charged with a mixture of $\mathrm{K}_{2} \mathrm{CO}_{3}\left(27.6 \mathrm{mg}, 0.2 \mathrm{mmol}, 1.0\right.$ equiv.), $\mathrm{Pd}_{2}(\mathrm{dba})_{3} \cdot \mathrm{CHCl}_{3}(5.2 \mathrm{mg}, 0.005 \mathrm{mmol}, 2.5 \mathrm{~mol} \%$ ), and $(S, S, S)$-SKP (6.1 mg, $0.01 \mathrm{mmol}, 5 \mathrm{~mol} \%)$ under an atmosphere of nitrogen. Toluene $(2 \mathrm{~mL})$ were added sequentially. The reaction mixture was stirred at $60{ }^{\circ} \mathrm{C}$ (oil bath) for $16 \mathrm{~h}$. After cooling to room temperature, the solvent was removed in vacuum. The residue was purified by flash column chromatography on silica gel to afford products 6 .

\section{Characterization of Products 6a-6e}

(S)-1-(5-hydroxy-2-phenylpent-1-en-3-yl)pyridin-2(1H)-one (6a) 


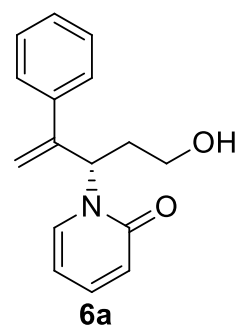

The crude product was purified by flash column chromatography (6:1 of EtOAc:Petroleum ether) on silica gel to provide the title compound; yield: $81 \%$ (41.2 mg); colorless liquid; $[\alpha]_{D}^{25}$ $=-183.284\left(c=0.35, \mathrm{CH}_{2} \mathrm{Cl}_{2}\right) ;{ }^{1} \mathrm{H} \mathrm{NMR}\left(500 \mathrm{MHz}, \mathrm{CDCl}_{3}\right) \delta 7.36(\mathrm{~d}, J=7.3 \mathrm{~Hz}, 1 \mathrm{H}), 7.25-$ $7.17(\mathrm{~m}, 4 \mathrm{H}), 6.54(\mathrm{~d}, J=9.1 \mathrm{~Hz}, 1 \mathrm{H}), 6.44(\mathrm{~d}, J=11.8 \mathrm{~Hz}, 1 \mathrm{H}), 6.12(\mathrm{t}, J=6.7 \mathrm{~Hz}, 1 \mathrm{H}), 5.76$ (s, 1H), $5.56(\mathrm{~d}, J=1.5 \mathrm{~Hz}, 1 \mathrm{H}), 4.35(\mathrm{dd}, J=10.3,2.9 \mathrm{~Hz}, 1 \mathrm{H}), 3.74(\mathrm{t}, J=8.2 \mathrm{~Hz}, 1 \mathrm{H}), 3.27$ $(\mathrm{t}, J=12.1 \mathrm{~Hz}, 1 \mathrm{H}), 2.50-2.43(\mathrm{~m}, 1 \mathrm{H}), 1.83-1.76(\mathrm{~m}, 2 \mathrm{H}) ;{ }^{13} \mathrm{C}$ NMR $\left(125 \mathrm{MHz}, \mathrm{CDCl}_{3}\right) \delta$ 163.8, 147.3, 139.6, 138.3, 133.5, 128.6, 128.3, 126.1, 120.1, 116.6, 107.7, 57.5, 51.9, 35.9; HRMS (ESI-TOF) m/z: $[\mathrm{M}+\mathrm{H}]^{+}$Calcd for $\mathrm{C}_{16} \mathrm{H}_{18} \mathrm{NO}_{2}{ }^{+}$256.1338; Found 256.1332; HPLC conditions: Chiralpak AS-H column, $254 \mathrm{~nm}$, flow rate: $0.7 \mathrm{~mL} / \mathrm{min}, i-\mathrm{PrOH} / \mathrm{hexanes}=1 / 3$, $\mathrm{t}_{\text {minor }}=10.57$ min, $\mathrm{t}_{\text {major }}=12.11 \mathrm{~min} ; 87 \%$ ee.

(S)-1-(5-hydroxy-2-phenylpent-1-en-3-yl)-5-methylpyridin-2(1H)-one (6b)<smiles>C=C(c1ccccc1)[C@H](CCO)N1C=C(C)[C+]=CC1=O</smiles>

The crude product was purified by flash column chromatography ( $7: 1$ of EtOAc:Petroleum ether) on silica gel to provide the title compound; yield: $82 \%(44.1 \mathrm{mg})$; colorless liquid; $[\alpha]_{D}^{25}$ $=-133.000\left(c=0.2, \mathrm{CH}_{2} \mathrm{Cl}_{2}\right) ;{ }^{1} \mathrm{H} \mathrm{NMR}\left(500 \mathrm{MHz}, \mathrm{CDCl}_{3}\right) \delta 7.36(\mathrm{~d}, J=7.3 \mathrm{~Hz}, 1 \mathrm{H}), 7.25-$ $7.19(\mathrm{~m}, 2 \mathrm{H}), 7.07(\mathrm{dd}, J=9.2,2.4 \mathrm{~Hz}, 1 \mathrm{H}), 6.92(\mathrm{~s}, 1 \mathrm{H}), 6.49(\mathrm{~d}, J=9.2 \mathrm{~Hz}, 1 \mathrm{H}), 6.40(\mathrm{~d}, J=$ $11.9 \mathrm{~Hz}, 1 \mathrm{H}), 5.77(\mathrm{~s}, 1 \mathrm{H}), 5.55(\mathrm{~d}, J=1.5 \mathrm{~Hz}, 1 \mathrm{H}), 4.47(\mathrm{~d}, J=6.9 \mathrm{~Hz}, 1 \mathrm{H}), 3.72(\mathrm{~s}, 1 \mathrm{H}), 3.25$ $(\mathrm{t}, J=11.7 \mathrm{~Hz}, 1 \mathrm{H}), 2.47-2.40(\mathrm{~m}, 1 \mathrm{H}), 1.95(\mathrm{~s}, 3 \mathrm{H}), 1.88(\mathrm{~s}, 1 \mathrm{H}), 1.83-1.77(\mathrm{~m}, 1 \mathrm{H}) ;{ }^{13} \mathrm{C}$ NMR $\left(125 \mathrm{MHz}, \mathrm{CDCl}_{3}\right) \delta 163.0,147.3,142.2,138.3,130.7,128.5,128.2,126.0,119.5,116.8,116.4$, 57.5, 51.7, 35.8, 17.2; HRMS (ESI-TOF) $\mathrm{m} / \mathrm{z}:[\mathrm{M}+\mathrm{Na}]^{+}$Calcd for $\mathrm{C}_{17} \mathrm{H}_{19} \mathrm{NO}_{2} \mathrm{Na}^{+}$292.1313; 
Found 292.1310; HPLC conditions: Chiralpak AD-H column, $254 \mathrm{~nm}$, flow rate: $0.8 \mathrm{~mL} / \mathrm{min}$, $i-\mathrm{PrOH} /$ hexanes $=1 / 9, \mathrm{t}_{\text {minor }}=19.08 \mathrm{~min}, \mathrm{t}_{\text {major }}=21.68 \mathrm{~min} ; 79 \%$ ee.

\section{(S)-1-(5-hydroxy-2-phenylpent-1-en-3-yl)-6-oxo-1,6-dihydropyridine-3-carbaldehyde}

(6c)<smiles>C=C(c1ccccc1)[C@@H](CCO)n1cc(C=O)ccc1=O</smiles>

The crude product was purified by flash column chromatography (5:1 of EtOAc:Petroleum ether) on silica gel to provide the title compound; yield: $84 \%$ (47.5 mg); colorless liquid; $[\alpha]_{D}^{25}$ $=-132.593\left(c=0.35, \mathrm{CH}_{2} \mathrm{Cl}_{2}\right) ;{ }^{1} \mathrm{H} \mathrm{NMR}\left(500 \mathrm{MHz}, \mathrm{CDCl}_{3}\right) \delta 9.51(\mathrm{~s}, 1 \mathrm{H}), 7.81(\mathrm{~d}, J=2.4 \mathrm{~Hz}$, 1H), $7.68(\mathrm{dd}, J=9.4,2.0 \mathrm{~Hz}, 1 \mathrm{H}), 7.34(\mathrm{~d}, J=7.0 \mathrm{~Hz}, 2 \mathrm{H}), 7.27-7.21(\mathrm{~m}, 2 \mathrm{H}), 6.57(\mathrm{~d}, J=$ $9.4 \mathrm{~Hz}, 1 \mathrm{H}), 6.43(\mathrm{~d}, J=10.6 \mathrm{~Hz}, 1 \mathrm{H}), 5.82(\mathrm{~s}, 1 \mathrm{H}), 5.63(\mathrm{~s}, 1 \mathrm{H}), 3.75(\mathrm{~d}, J=9.9 \mathrm{~Hz}, 1 \mathrm{H}), 3.63$ $(\mathrm{s}, 1 \mathrm{H}), 3.39(\mathrm{td}, J=11.7,2.7 \mathrm{~Hz}, 1 \mathrm{H}), 2.54-2.47(\mathrm{~m}, 1 \mathrm{H}), 2.16(\mathrm{~s}, 1 \mathrm{H}), 1.95-1.89(\mathrm{~m}, 1 \mathrm{H}) ;{ }^{13} \mathrm{C}$ $\operatorname{NMR}\left(125 \mathrm{MHz}, \mathrm{CDCl}_{3}\right) \delta 185.9,163.6,146.4,142.6,137.7,135.8,128.7,128.5,125.9,120.2$, 119.2, 117.5, 57.6, 53.0, 35.9; HRMS (ESI-TOF) m/z: $[\mathrm{M}+\mathrm{Na}]^{+} \mathrm{Calcd}$ for $\mathrm{C}_{17} \mathrm{H}_{17} \mathrm{NO}_{3} \mathrm{Na}^{+}$ 306.1106; Found 306.1104; HPLC conditions: Chiralpak AD-H column, 254 nm, flow rate: 0.7 $\mathrm{mL} / \mathrm{min}, i-\mathrm{PrOH} /$ hexanes $=1 / 3, \mathrm{t}_{\text {minor }}=28.35 \mathrm{~min}, \mathrm{t}_{\text {major }}=32.75 \mathrm{~min} ; 83 \%$ ee.

(S)-1-(5-hydroxy-2-(4-methoxyphenyl)pent-1-en-3-yl)pyridin-2(1H)-one (6d)<smiles>C=C(c1ccc(OC)cc1)C(CCO)N1C=C[Ge]c2ccccc21</smiles>

The crude product was purified by flash column chromatography (6:1 of EtOAc:Petroleum ether) on silica gel to provide the title compound; yield: $78 \%$ (44.4 mg); colorless liquid; $[\alpha]_{D}^{25}$ $=-90.000\left(c=0.2, \mathrm{CH}_{2} \mathrm{Cl}_{2}\right) ;{ }^{1} \mathrm{H} \mathrm{NMR}\left(500 \mathrm{MHz}, \mathrm{CDCl}_{3}\right) \delta 7.30(\mathrm{~d}, J=8.7 \mathrm{~Hz}, 2 \mathrm{H}), 7.25-7.21$ (m, 1H), $7.18(\mathrm{~d}, J=6.9 \mathrm{~Hz}, 1 \mathrm{H}), 6.77(\mathrm{~d}, J=8.7 \mathrm{~Hz}, 2 \mathrm{H}), 6.55(\mathrm{~d}, J=9.0 \mathrm{~Hz}, 1 \mathrm{H}), 6.39(\mathrm{~d}, J$ 
$=11.7 \mathrm{~Hz}, 1 \mathrm{H}), 6.13(\mathrm{t}, J=6.7 \mathrm{~Hz}, 1 \mathrm{H}), 5.69(\mathrm{~s}, 1 \mathrm{H}), 5.47(\mathrm{~s}, 1 \mathrm{H}), 4.35(\mathrm{~s}, 1 \mathrm{H}), 3.74(\mathrm{~s}, 4 \mathrm{H})$, $3.27(\mathrm{t}, J=11.7 \mathrm{~Hz}, 1 \mathrm{H}), 2.48-2.41(\mathrm{~m}, 1 \mathrm{H}), 1.86(\mathrm{~s}, 1 \mathrm{H}), 1.79(\mathrm{t}, J=12.8 \mathrm{~Hz}, 1 \mathrm{H}) ;{ }^{13} \mathrm{C} \mathrm{NMR}$ $\left(125 \mathrm{MHz}, \mathrm{CDCl}_{3}\right) \delta 163.8,159.6,146.5,139.6,133.5,130.6,127.2,120.0,114.9,113.9,107.8$, 57.5, 55.1, 51.9, 35.9; HRMS (ESI-TOF) m/z: $[\mathrm{M}+\mathrm{Na}]^{+}$Calcd for $\mathrm{C}_{17} \mathrm{H}_{19} \mathrm{NO}_{3} \mathrm{Na}^{+}$308.1263; Found 308.1261; HPLC conditions: Chiralpak OD-H column, $254 \mathrm{~nm}$, flow rate: $1.0 \mathrm{~mL} / \mathrm{min}$, $i-\mathrm{PrOH} /$ hexanes $=3 / 17, \mathrm{t}_{\text {minor }}=14.41 \mathrm{~min}, \mathrm{t}_{\text {major }}=21.77 \mathrm{~min} ; 82 \%$ ee.

(S)-1-(2-(4-bromophenyl)-5-hydroxypent-1-en-3-yl)pyridin-2(1H)-one (6e)<smiles></smiles>

The crude product was purified by flash column chromatography (5:1 of EtOAc:Petroleum ether) on silica gel to provide the title compound; yield: $86 \%$ (57.2 mg); colorless liquid; $[\alpha]_{D}^{25}$ $=-172.667\left(c=0.45, \mathrm{CH}_{2} \mathrm{Cl}_{2}\right) ;{ }^{1} \mathrm{H} \mathrm{NMR}\left(500 \mathrm{MHz}, \mathrm{CDCl}_{3}\right) \delta 7.37(\mathrm{~d}, J=8.5 \mathrm{~Hz}, 2 \mathrm{H}), 7.26-$ $7.21(\mathrm{~m}, 3 \mathrm{H}), 7.14(\mathrm{dd}, J=6.9,1.7 \mathrm{~Hz}, 1 \mathrm{H}), 6.55(\mathrm{~d}, J=9.1 \mathrm{~Hz}, 1 \mathrm{H}), 6.39(\mathrm{~d}, J=11.7 \mathrm{~Hz}, 1 \mathrm{H})$, $6.14(\mathrm{t}, J=6.7 \mathrm{~Hz}, 1 \mathrm{H}), 5.76(\mathrm{~s}, 1 \mathrm{H}), 5.58(\mathrm{~d}, J=1.4 \mathrm{~Hz}, 1 \mathrm{H}), 4.25(\mathrm{~s}, 1 \mathrm{H}), 3.73(\mathrm{dd}, J=12.3$, $2.8 \mathrm{~Hz}, 1 \mathrm{H}), 3.26(\mathrm{td}, J=12.2,1.9 \mathrm{~Hz}, 1 \mathrm{H}), 2.48-2.41(\mathrm{~m}, 1 \mathrm{H}), 1.83-1.77(\mathrm{~m}, 1 \mathrm{H}) ;{ }^{13} \mathrm{C}$ NMR $\left(125 \mathrm{MHz}, \mathrm{CDCl}_{3}\right) \delta 163.7,146.3,139.8,137.1,133.2,131.7,127.7,122.4,120.1,117.0,107.9$, 57.4, 51.6, 35.7; HRMS (ESI-TOF) m/z: [M + Na] ${ }^{+}$Calcd for $\mathrm{C}_{16} \mathrm{H}_{17} \mathrm{BrNO}_{2}{ }^{+}$334.0433; Found 334.0436; HPLC conditions: Chiralpak AS-H column, $254 \mathrm{~nm}$, flow rate: $0.5 \mathrm{~mL} / \mathrm{min}, i$ $\mathrm{PrOH} /$ hexanes $=2 / 8, \mathrm{t}_{\text {minor }}=18.81 \mathrm{~min}, \mathrm{t}_{\mathrm{major}}=21.90 \mathrm{~min} ; 77 \%$ ee.

\section{General procedures for the hydrogenation of 4aa}
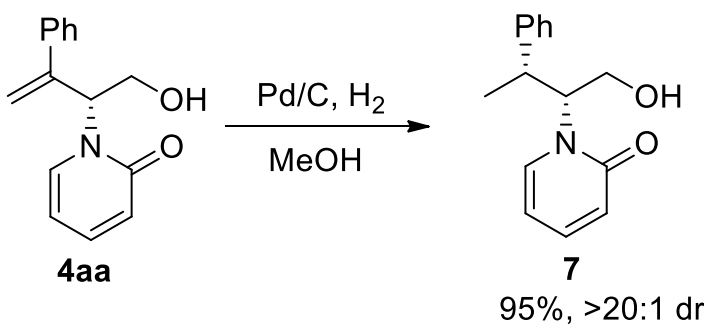
To a $5 \mathrm{~mL}$ reaction vial containing a stirring bar, 4 aa $(0.2 \mathrm{mmol})$ in $\mathrm{MeOH}(1.0 \mathrm{~mL})$ was added. Then, $\mathrm{Pd} / \mathrm{C}$ (4.3 mg, $20 \mathrm{~mol} \%)$ was added to the reaction mixture. The reaction mixture was vigorously stirred in the presence of hydrogen gas at room temperature for 12 hours. The precipitate was filtered out with celite and washed with ethanol, and the filtrate was removed in vacuum. The residue was purified by flash column chromatography on silica gel to afford product 7 (46 mg, 95\%).

\section{Characterization of products 7}

\section{1-((2R)-1-hydroxy-3-phenylbutan-2-yl)pyridin-2(1H)-one (7)}

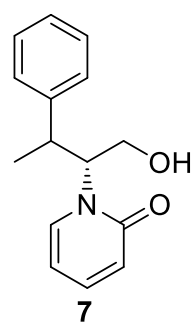

The crude product was purified by flash column chromatography (7:1 of EtOAc:Petroleum ether) on silica gel to provide the title compound; White solid; $178-180{ }^{\circ} \mathrm{C} ;{ }^{1} \mathrm{H} \mathrm{NMR}(500 \mathrm{MHz}$, $\left.\mathrm{CDCl}_{3}\right) \delta 7.52(\mathrm{~d}, J=6.2 \mathrm{~Hz}, 1 \mathrm{H}), 7.37-7.30(\mathrm{~m}, 5 \mathrm{H}), 7.28-7.24(\mathrm{~m}, 1 \mathrm{H}), 6.59(\mathrm{~d}, J=9.1 \mathrm{~Hz}$, $1 \mathrm{H}), 6.25(\mathrm{t}, J=6.6 \mathrm{~Hz}, 1 \mathrm{H}), 5.04-4.88(\mathrm{~m}, 1 \mathrm{H}), 3.67-3.58(\mathrm{~m}, 2 \mathrm{H}), 3.43(\mathrm{~s}, 1 \mathrm{H}), 1.10(\mathrm{~d}, J=$ $7.0 \mathrm{~Hz}, 3 \mathrm{H}) ;{ }^{13} \mathrm{C} \mathrm{NMR}\left(125 \mathrm{MHz}, \mathrm{CDCl}_{3}\right) \delta 163.9,143.0,139.2,136.2,128.8,127.6,127.1$, 120.8, 106.4, 62.5, 39.6, 29.7, 18.9; HRMS (ESI-TOF) m/z: $[\mathrm{M}+\mathrm{Na}]^{+}$Calcd for $\mathrm{C}_{15} \mathrm{H}_{17} \mathrm{NO}_{2} \mathrm{Na}^{+}$ 266.1157; Found 266.1147.

\section{General Procedures for the Synthesis of 2-pyridone compound 10}
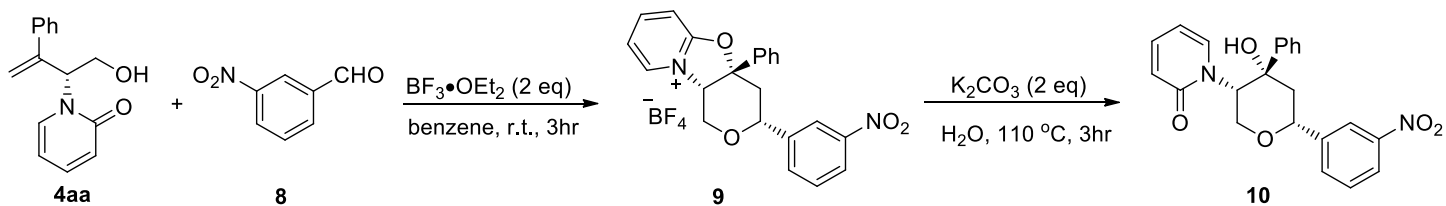

To a stirring solution of 3-nitrobenzaldehyde $(83.0 \mathrm{mg}, 0.55 \mathrm{mmol})$ with boron trifluoride etherate $(141 \mathrm{mg}, 1.0 \mathrm{mmol})$ in benzene $(1.0 \mathrm{~mL})$ at room temperature was added compound 4aa $(120.5 \mathrm{mg}, 0.5 \mathrm{mmol})$ in benzene $(4 \mathrm{~mL})$ drop by drop over $5 \mathrm{~min}$. The reaction mixture was stirred at the same temperature for 3 hour. The reaction mixture was quenched with saturated sodium bicarbonate aqueous solution. The product was extracted with ethyl acetate, and then washed with brine and cold water. The organic layer was dried over $\mathrm{Na}_{2} \mathrm{SO}_{4}$ and 
evaporated to leave the crude product. The crude product washed with $25 \%$ ethyl acetate and hexane to remove remaining 3-nitrobenzaldehyde to get pure compound 9 in $75 \%$ yield.

Compound 9 (1 equiv.) was slightly dissolved in hot water, and $\mathrm{K}_{2} \mathrm{CO}_{3}$ (2 equiv.) was added. The mixture was boiled (oil bath) for 3 hours. After cooling the product was extracted by ethyl acetate, and the solvent was removed on a rotary evaporator. The crude product was purified by column chromatography on silica (ethyl acetate:petroleum ether $=1: 2$ ) to give compound $\mathbf{1 0}$ in $85 \%$ yield.

\section{Characterization of products 9 and 10}

(3R,4aS,10aS)-3-(3-nitrophenyl)-4a-phenyl-3,4,4a,10a-tetrahydro-1H-

pyrano[3',4':4,5] oxazolo[3,2-a]pyridin-10-ium tetrafluoroborate (9)

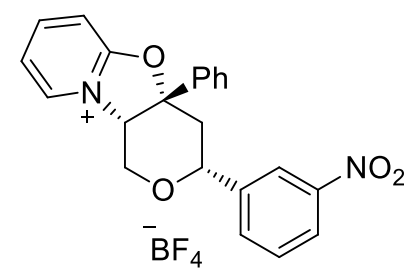

9

Yield: 75\% (173.4 mg); white solid; 255-258 ${ }^{\circ} \mathrm{C} ;{ }^{1} \mathrm{H}$ NMR (500 MHz, DMSO) $\delta 9.09$ (d, $\mathrm{J}=6.2 \mathrm{~Hz}, 1 \mathrm{H}), 8.61-8.57(\mathrm{~m}, 1 \mathrm{H}), 8.16(\mathrm{dd}, \mathrm{J}=8.2,1.8 \mathrm{~Hz}, 1 \mathrm{H}), 8.12(\mathrm{~s}, 1 \mathrm{H}), 7.84-7.78(\mathrm{~m}$, 4H), $7.72(\mathrm{~d}, \mathrm{~J}=8.8 \mathrm{~Hz}, 1 \mathrm{H}), 7.64(\mathrm{t}, \mathrm{J}=8.0 \mathrm{~Hz}, 1 \mathrm{H}), 7.59-7.52(\mathrm{~m}, 3 \mathrm{H}), 5.60(\mathrm{~s}, 1 \mathrm{H}), 5.08(\mathrm{~d}$, $\mathrm{J}=14.7 \mathrm{~Hz}, 1 \mathrm{H}), 5.02(\mathrm{dd}, \mathrm{J}=8.5,4.0 \mathrm{~Hz}, 1 \mathrm{H}), 4.47(\mathrm{dd}, \mathrm{J}=14.8,2.2 \mathrm{~Hz}, 1 \mathrm{H}), 3.13(\mathrm{dd}, \mathrm{J}=$ 15.0, 4.2 Hz, 1H), $2.55(\mathrm{dd}, \mathrm{J}=15.0,8.6 \mathrm{~Hz}, 1 \mathrm{H}) ;{ }^{13} \mathrm{C} \mathrm{NMR}(125 \mathrm{MHz}, \mathrm{DMSO}) \delta 158.9,149.6$, $147.7,143.1,137.2,137.0,132.8,129.9,129.8,129.0,126.3,122.7,120.5,120.4,111.5,91.0$, 73.2, 65.6, 62.7, 38.0; HRMS (ESI-TOF) m/z: [M] Calcd for $\mathrm{C}_{22} \mathrm{H}_{19} \mathrm{~N}_{2} \mathrm{O}_{4}{ }^{+}$375.1339; Found 375.1338 .

\section{1-((3S,4S,6R)-4-hydroxy-6-(3-nitrophenyl)-4-phenyltetrahydro-2H-pyran-3-yl)pyridin-} 2(1H)-one (10)

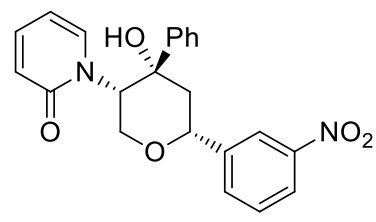

10

The crude product was purified by flash column chromatography (1:2 of EtOAc:Petroleum ether) on silica gel to provide the title compound; yield: $85 \%$ (125.0 mg); white solid; 158 - 
$160{ }^{\circ} \mathrm{C} ;{ }^{1} \mathrm{H}$ NMR $\left(500 \mathrm{MHz}, \mathrm{CDCl}_{3}\right) \delta 8.56(\mathrm{~s}, 1 \mathrm{H}), 8.32(\mathrm{~s}, 1 \mathrm{H}), 8.20(\mathrm{dd}, \mathrm{J}=8.2,1.1 \mathrm{~Hz}, 1 \mathrm{H})$, $7.83(\mathrm{~s}, 2 \mathrm{H}), 7.77(\mathrm{~d}, \mathrm{~J}=7.6 \mathrm{~Hz}, 1 \mathrm{H}), 7.60(\mathrm{t}, \mathrm{J}=8.0 \mathrm{~Hz}, 1 \mathrm{H}), 7.52-7.39(\mathrm{~m}, 4 \mathrm{H}), 6.66(\mathrm{~d}, \mathrm{~J}=$ $8.3 \mathrm{~Hz}, 1 \mathrm{H}), 6.39(\mathrm{~s}, 1 \mathrm{H}), 5.70(\mathrm{~s}, 1 \mathrm{H}), 4.88(\mathrm{~d}, \mathrm{~J}=9.1 \mathrm{~Hz}, 1 \mathrm{H}), 4.48-4.41(\mathrm{~m}, 2 \mathrm{H}), 4.06(\mathrm{~d}, \mathrm{~J}$ $=11.9 \mathrm{~Hz}, 1 \mathrm{H}), 2.76(\mathrm{~d}, \mathrm{~J}=14.5 \mathrm{~Hz}, 1 \mathrm{H}), 2.18(\mathrm{~s}, 1 \mathrm{H}) ;{ }^{13} \mathrm{C} \mathrm{NMR}\left(125 \mathrm{MHz}, \mathrm{CDCl}_{3}\right) \delta 166.0$, $148.4,143.2,142.9,140.0,136.3,131.7,129.6,129.2,128.4,126.0,122.9,120.8,120.4,107.4$, 75.7, 73.2, 67.4, 56.7, 38.6; HRMS (ESI-TOF) m/z: $[\mathrm{M}+\mathrm{H}]^{+}$Calcd for $\mathrm{C}_{22} \mathrm{H}_{21} \mathrm{~N}_{2} \mathrm{O}_{5}{ }^{+}$393.1450; Found 393.1442; HPLC conditions: Chiralpak AS-H column, $254 \mathrm{~nm}$, flow rate: $0.7 \mathrm{~mL} / \mathrm{min}$, $\mathrm{i}-\mathrm{PrOH} /$ hexanes $=7 / 13, \mathrm{t}_{\text {major }}=27.92 \mathrm{~min}, \mathrm{t}_{\mathrm{minor}}=34.87 \mathrm{~min} ; 92 \%$ ee.

\section{X-Rays Crystallography of 4aa (CCDC2036007)}

A single crystal of $4 \mathbf{a a}$ was obtained from ethyl acetate/ $n$-hexane solvent at room temperature. Diffraction data were collected on Bruker SMART Apex-III CMOS-Based X-ray diffractometer with $\mathrm{Cu}-\mathrm{K} \alpha$. Radiation $(\lambda=1.54178)$. The empirical absorption correction was applied by using the SADABS program. The structure was solved using direct method, and refined by full matrix least-squares on $\mathrm{F}^{2}$ (G.M Sheldrick, SHELXTL2014, program of crystal structure refinement, University of Göttingen, Germany).

Table S3. Crystal Data and Structure Refinement for 4aa.

Identification code

Empirical formula

Formula weight

Temperature

Wavelength

Crystal system, space group

Unit cell dimensions

Volume

Z, Calculated density

Absorption coefficient
$4 \mathbf{a a}$

C15 H15 N O2

241.28

297(2) K

$1.54178 \mathrm{~A}$

Orthorhombic, Pca2(1)

$\mathrm{a}=7.042(2) \mathrm{A} \quad$ alpha $=90.00(3) \mathrm{deg}$

$\mathrm{b}=8.921(2) \mathrm{A} \quad$ beta $=90.00(3) \mathrm{deg}$.

$\mathrm{c}=20.360(4) \mathrm{A} \quad$ gamma $=90.00(3) \mathrm{deg}$

$1279.0(5) \mathrm{A}^{\wedge} 3$

4, $\quad 1.253 \mathrm{Mg} / \mathrm{m}^{\wedge} 3$

$0.669 \mathrm{~mm}^{\wedge}-1$ 
$\mathrm{F}(000)$

Crystal size

Theta range for data collection

Limiting indices

Reflections collected / unique

Completeness to theta $=67.679$

Refinement method

Data / restraints / parameters

Goodness-of-fit on $\mathrm{F}^{\wedge} 2$

Final $\mathrm{R}$ indices [I $>2 \operatorname{sigma}(\mathrm{I})]$

$\mathrm{R}$ indices (all data)

Absolute structure parameter

Extinction coefficient

Largest diff. peak and hole
512

$0.200 \times 0.180 \times 0.150 \mathrm{~mm}$

4.957 to $68.176 \mathrm{deg}$.

$-8<=\mathrm{h}<=8,-10<=\mathrm{k}<=10,-24<=\mathrm{l}<=24$

$13667 / 2330[\mathrm{R}(\mathrm{int})=0.0414]$

$99.3 \%$

Full-matrix least-squares on $\mathrm{F}^{\wedge} 2$

$2330 / 1 / 165$

1.072

$\mathrm{R} 1=0.0291, \mathrm{wR} 2=0.0733$

$\mathrm{R} 1=0.0309, \mathrm{wR} 2=0.0752$

$-0.01(9)$

$\mathrm{n} / \mathrm{a}$

0.099 and -0.103 e. $\mathrm{A}^{\wedge}-3$

Figure S1. Molecular structure of 4aa (ellipsoid contour probability level of 50\%)

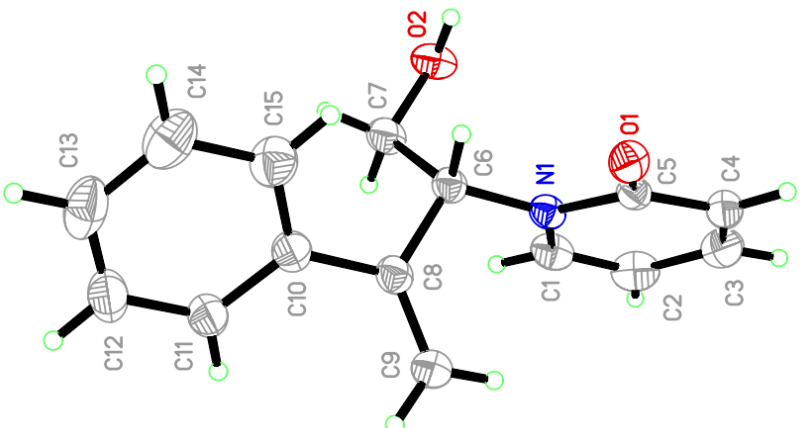

\section{X-rays crystallography of 7 (CCDC2036008)}

A single crystal of 10 was obtained from EA/n-Hexane solvent at room temperature. Diffraction data were collected on Bruker SMART Apex-III CMOS-Based X-ray diffractometer with $\mathrm{Cu}-$ Ka. Radiation $(\lambda=1.54178)$. The empirical absorption correction was applied by using the SADABS program. The structure was solved using direct method, and refined by full matrix least-squares on F2 (G.M Sheldrick, SHELXTL2014, program of crystal structure refinement, University of Göttingen, Germany). 
Table S4. Crystal data and structure refinement for 7 .

Identification code $\quad 7$

Empirical formula $\quad \mathrm{C} 15 \mathrm{H} 17 \mathrm{~N} \mathrm{O} 2$

Formula weight $\quad 243.29$

$\begin{array}{ll}\text { Temperature } & 173(2) \mathrm{K}\end{array}$

Wavelength $\quad 1.54178 \mathrm{~A}$

Crystal system, space group $\quad$ Monoclinic, P2(1)

Unit cell dimensions $\quad \mathrm{a}=5.89870(10) \mathrm{A} \quad \mathrm{alpha}=90 \mathrm{deg}$.

$\mathrm{b}=17.2640(4) \mathrm{A} \quad$ beta $=114.3040(10)$ deg.

$\mathrm{c}=6.7917(2) \mathrm{A} \quad$ gamma $=90 \mathrm{deg}$.

Volume $\quad 630.34(3) \mathrm{A}^{\wedge} 3$

$\mathrm{Z}$, Calculated density $\quad 2, \quad 1.282 \mathrm{Mg} / \mathrm{m}^{\wedge} 3$

Absorption coefficient $\quad 0.679 \mathrm{~mm}^{\wedge}-1$

$\mathrm{F}(000) \quad 260$

$\begin{array}{ll}\text { Crystal size } & 0.200 \times 0.180 \times 0.160 \mathrm{~mm}\end{array}$

Theta range for data collection $\quad 7.154$ to $68.256 \mathrm{deg}$.

Limiting indices
\[ -7<=\mathrm{h}<=7,-20<=\mathrm{k}<=20,-8<=1<=8 \]

Reflections collected / unique $\quad 6201 / 2261[\mathrm{R}(\mathrm{int})=0.0317]$

Completeness to theta $=67.679 \quad 98.9 \%$

Refinement method $\quad$ Full-matrix least-squares on $\mathrm{F}^{\wedge} 2$

Data / restraints / parameters $\quad 2261 / 1 / 166$

Goodness-of-fit on $\mathrm{F}^{\wedge} 2$

Final R indices $[\mathrm{I}>2 \operatorname{sigma}(\mathrm{I})] \quad \mathrm{R} 1=0.0421, \mathrm{wR} 2=0.1103$

$\mathrm{R}$ indices (all data) $\quad \mathrm{R} 1=0.0427, \mathrm{wR} 2=0.1108$

Absolute structure parameter $\quad 0.05(9)$

Extinction coefficient $\quad$ n/a

Largest diff. peak and hole $\quad 0.417$ and -0.184 e. $\mathrm{A}^{\wedge}-3$

Figure S2: Molecular structure of 7 (ellipsoid contour probability level of 50\%) 


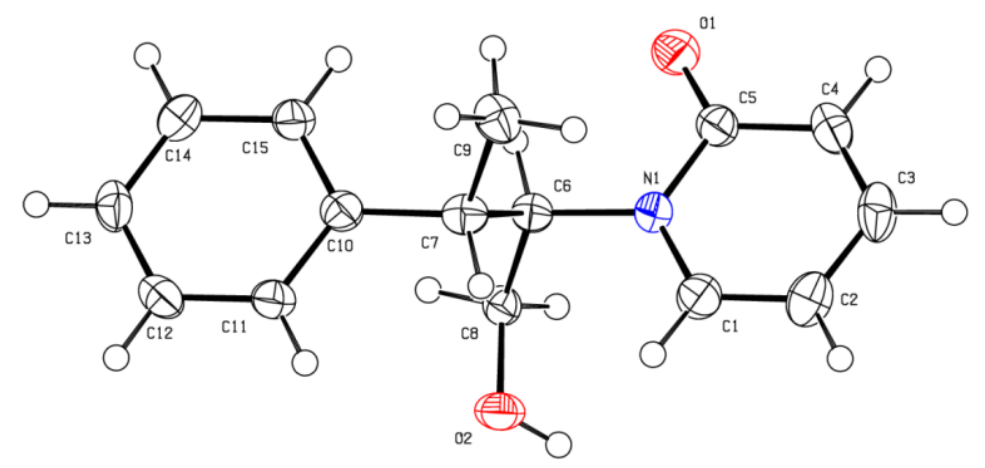

\section{X-Rays Crystallography of 9(CCDC2061837)}

A single crystal of 12 was obtained from Ethanol/n-Hexane solvent at room temperature. Diffraction data were collected on Bruker SMART Apex-III CMOS-Based X-ray diffractometer with $\mathrm{Cu}-\mathrm{K} \alpha$. Radiation $(\lambda=1.54178)$. The empirical absorption correction was applied by using the SADABS program. The structure was solved using direct method, and refined by full matrix least-squares on F2 (G.M Sheldrick, SHELXTL2014, program of crystal structure refinement, University of Göttingen, Germany).

Table S5. Crystal data and structure refinement for 9.

Identification code

Empirical formula

Formula weight

Temperature

Wavelength

Crystal system, space group

Unit cell dimensions

Volume

Z, Calculated density

Absorption coefficient

$\mathrm{F}(000)$

\section{9}

C22 H19 B F4 N2 O4

462.20

297(2) K

$1.54178 \mathrm{~A}$

Monoclinic, P2(1)/c

$\mathrm{a}=16.3522(3) \mathrm{A} \quad$ alpha $=90 \mathrm{deg}$.

$\mathrm{b}=9.9455(3) \mathrm{A} \quad$ beta $=111.7920(10) \mathrm{deg}$.

$\mathrm{c}=13.8666(3) \mathrm{A} \quad$ gamma $=90 \mathrm{deg}$.

2093.98(9) $\mathrm{A}^{\wedge} 3$

4, $\quad 1.466 \mathrm{Mg} / \mathrm{m}^{\wedge} 3$

$1.067 \mathrm{~mm}^{\wedge}-1$

952 
Crystal size

Theta range for data collection

Limiting indices

Reflections collected / unique

Completeness to theta $=67.679$

Refinement method

Data / restraints / parameters

Goodness-of-fit on $\mathrm{F}^{\wedge} 2$

Final $\mathrm{R}$ indices [I $>2 \operatorname{sigma}(\mathrm{I})]$

$\mathrm{R}$ indices (all data)

Absolute structure parameter

Extinction coefficient

Largest diff. peak and hole
$0.180 \times 0.160 \times 0.140 \mathrm{~mm}$

2.910 to $68.270 \mathrm{deg}$.

$-19<=\mathrm{h}<=19,-11<=\mathrm{k}<=11,-16<=1<=15$

$17362 / 3827[\mathrm{R}(\mathrm{int})=0.0499]$

$99.8 \%$

Full-matrix least-squares on $\mathrm{F}^{\wedge} 2$

$3827 / 0 / 298$

1.048

$\mathrm{R} 1=0.0665, \mathrm{wR} 2=0.1764$

$\mathrm{R} 1=0.0906, \mathrm{wR} 2=0.1965$

$0.05(9)$

$\mathrm{n} / \mathrm{a}$

0.590 and -0.445 e. $\mathrm{A}^{\wedge}-3$

Figure S3: Molecular structure of 12 (ellipsoid contour probability level of 50\%)

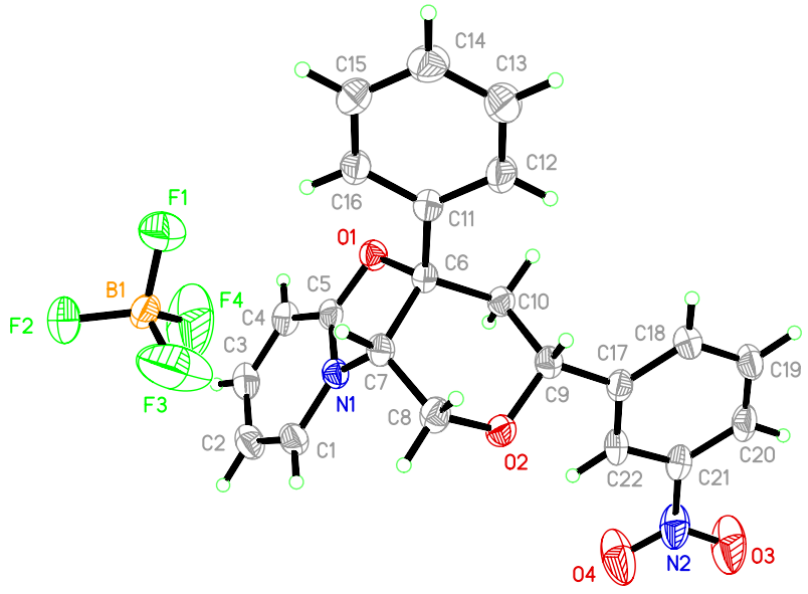

\section{References}

1. Luo, H.; Ma, S. CuI-catalyzed synthesis of functionalized terminal allenes from 1-alkynes. Eur. J. Org. Chem. 2013, 15, 3041-3048.

2. Clavier, H.; Jeune, K. L.; Riggi, I. D.; Tenaglia, A.; Buono, G. Highly selective cobalt-mediated [6+2] cycloaddition of cycloheptatriene and allenes. Org. Lett. 2011, 13, 308-311.

3. Steib, P.; Breit, B. Enantioselective rhodium-catalyzed dimerization of $\omega$-allenyl carboxylic acids: straightforward synthesis of $\mathrm{C}_{2}$-symmetric macrodiolides. Angew. Chem. Int. Ed. 2018, 57,65726576.

4. Barbot, F.; Dauphin, B.; Miginiac, P. An easy preparation of $\alpha$-allenic ethers and tertiary $\alpha$-allenic amines. Synthesis 1985, 8, 768-770. 
5. Hurtado-Rodrigo, C.; Hoehne, S.; Muñoz, M. P. A new gold-catalysed azidation of allenes. Chem. Commun. 2014, 50, 1494-1496. 


\section{Copies of ${ }^{1} \mathrm{H}$ and ${ }^{13} \mathrm{C}$ NMR Spectra}

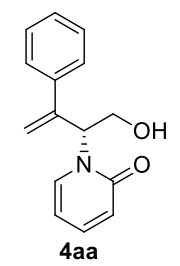

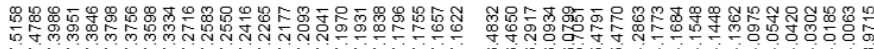

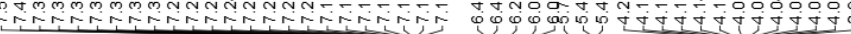

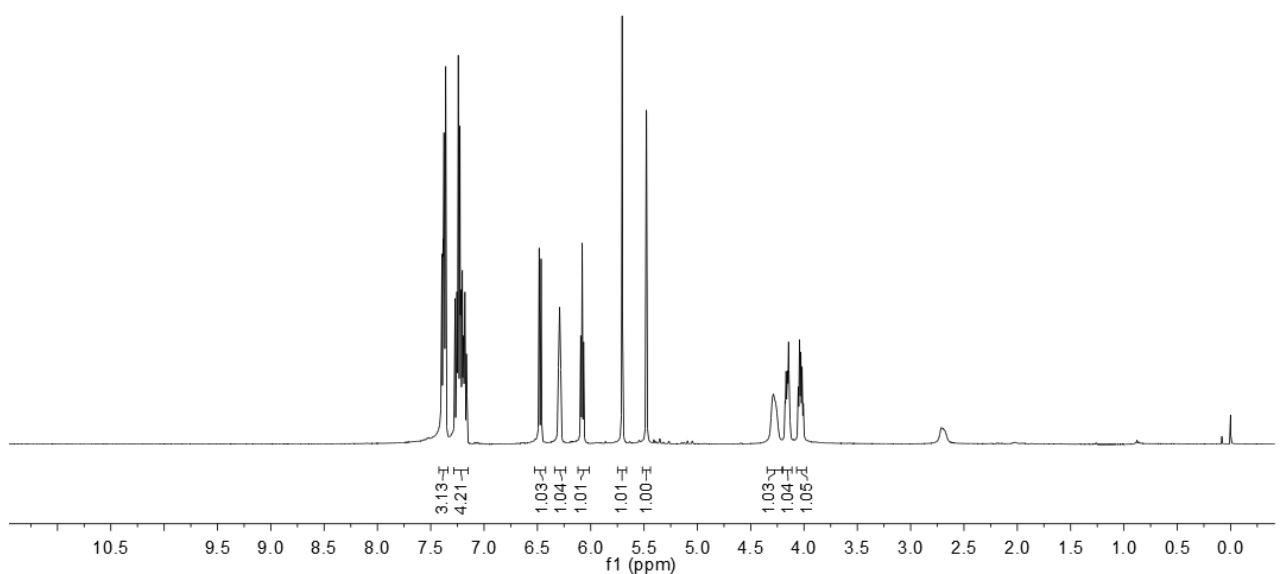

${ }^{1} \mathrm{H}$ NMR spectrum $\left(500 \mathrm{MHz}, \mathrm{CDCl}_{3}\right)$ of compound $\mathbf{4 a a}$

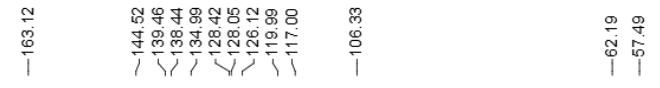

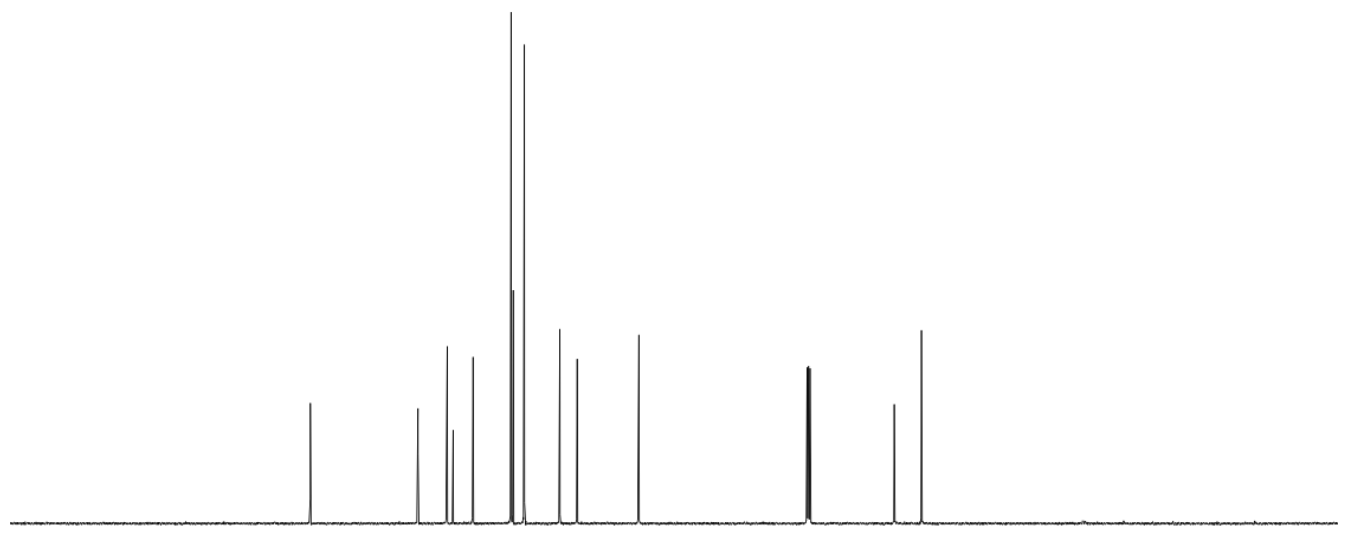

\begin{tabular}{llllllllllllllllllllllllllllllll}
\hline 210 & 200 & 190 & 180 & 170 & 160 & 150 & 140 & 130 & 120 & 110 & 100 & 90 & 80 & 70 & 60 & 50 & 40 & 30 & 20 & 10 & 0 & -10
\end{tabular}

${ }^{13} \mathrm{C}$ NMR spectrum $\left(125 \mathrm{MHz}, \mathrm{CDCl}_{3}\right.$ ) of compound $4 \mathbf{a a}$ 

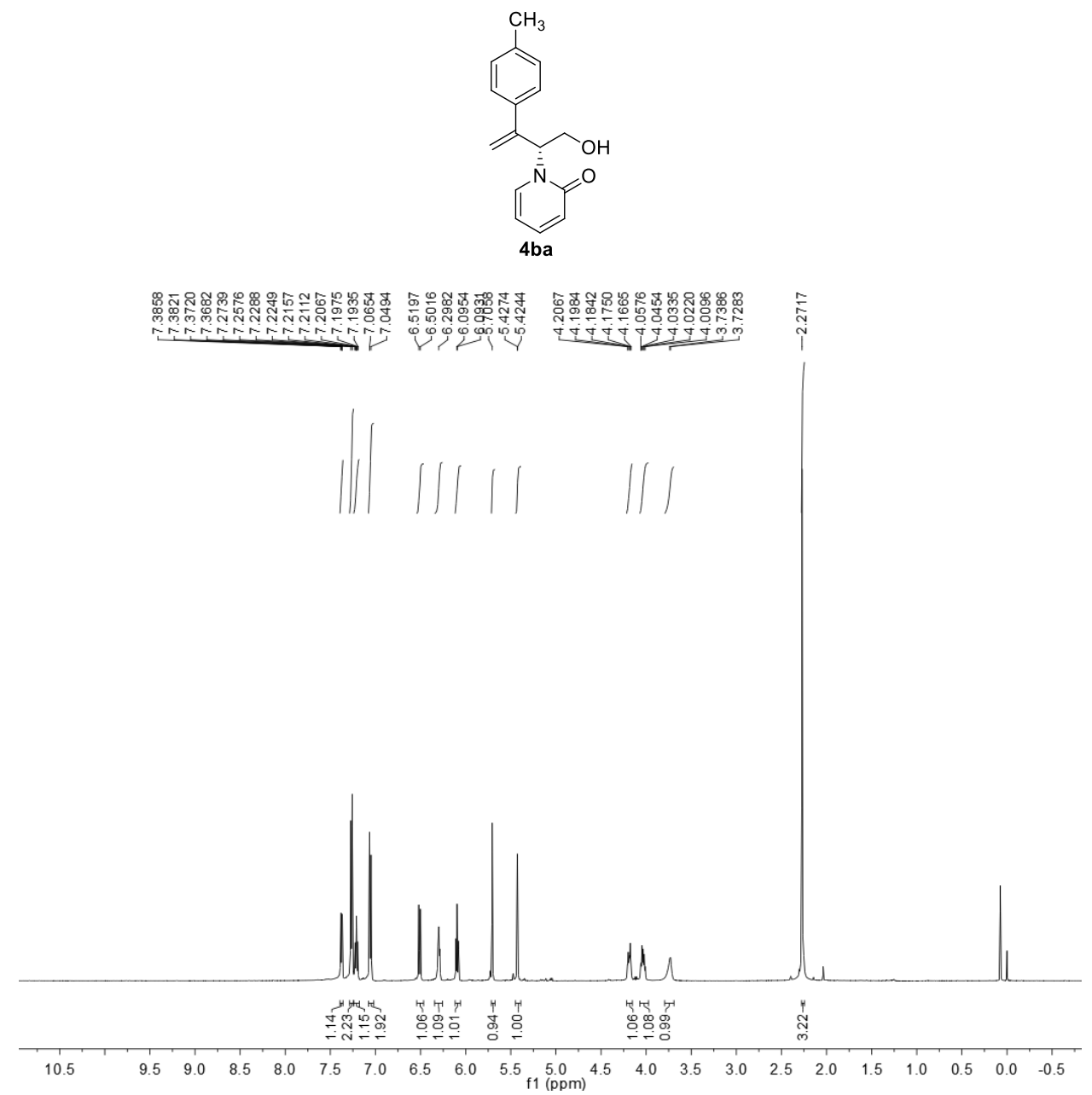

${ }^{1} \mathrm{H}$ NMR spectrum $\left(500 \mathrm{MHz}, \mathrm{CDCl}_{3}\right)$ of compound $\mathbf{4 b a}$

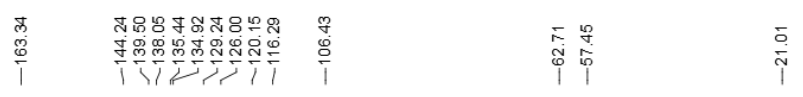

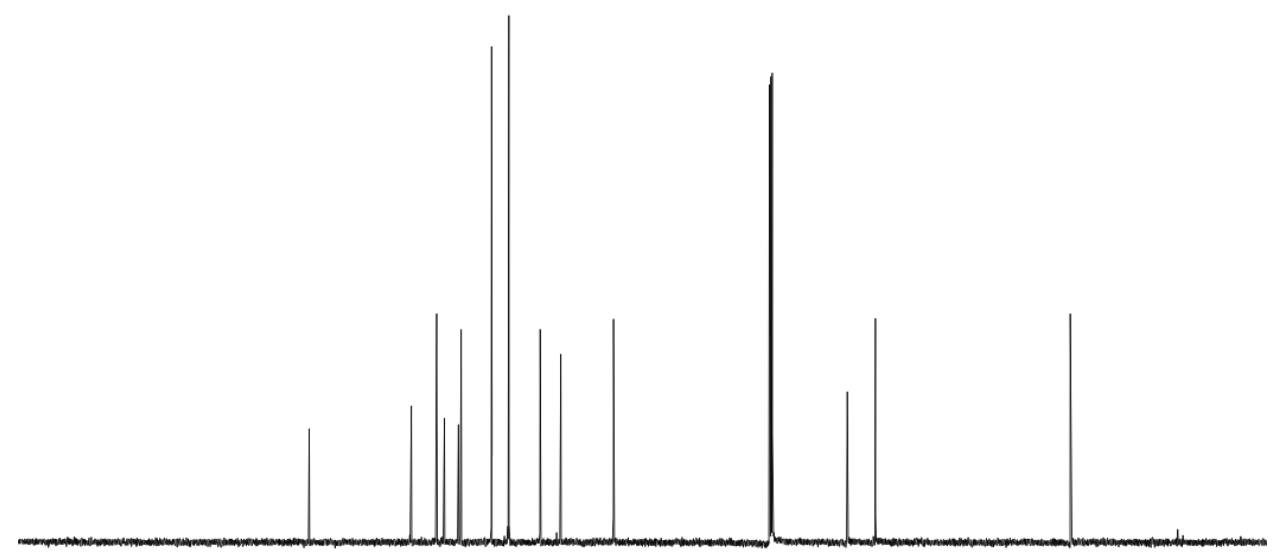

$\begin{array}{lllllllllllllllllllllll}210 & 200 & 190 & 180 & 170 & 160 & 150 & 140 & 130 & 120 & 110 & 100 & 90 & 80 & 70 & 60 & 50 & 40 & 30 & 20 & 10 & 0 & -10\end{array}$

${ }^{13} \mathrm{C}$ NMR spectrum $\left(125 \mathrm{MHz}, \mathrm{CDCl}_{3}\right)$ of compound $4 \mathbf{b a}$ 


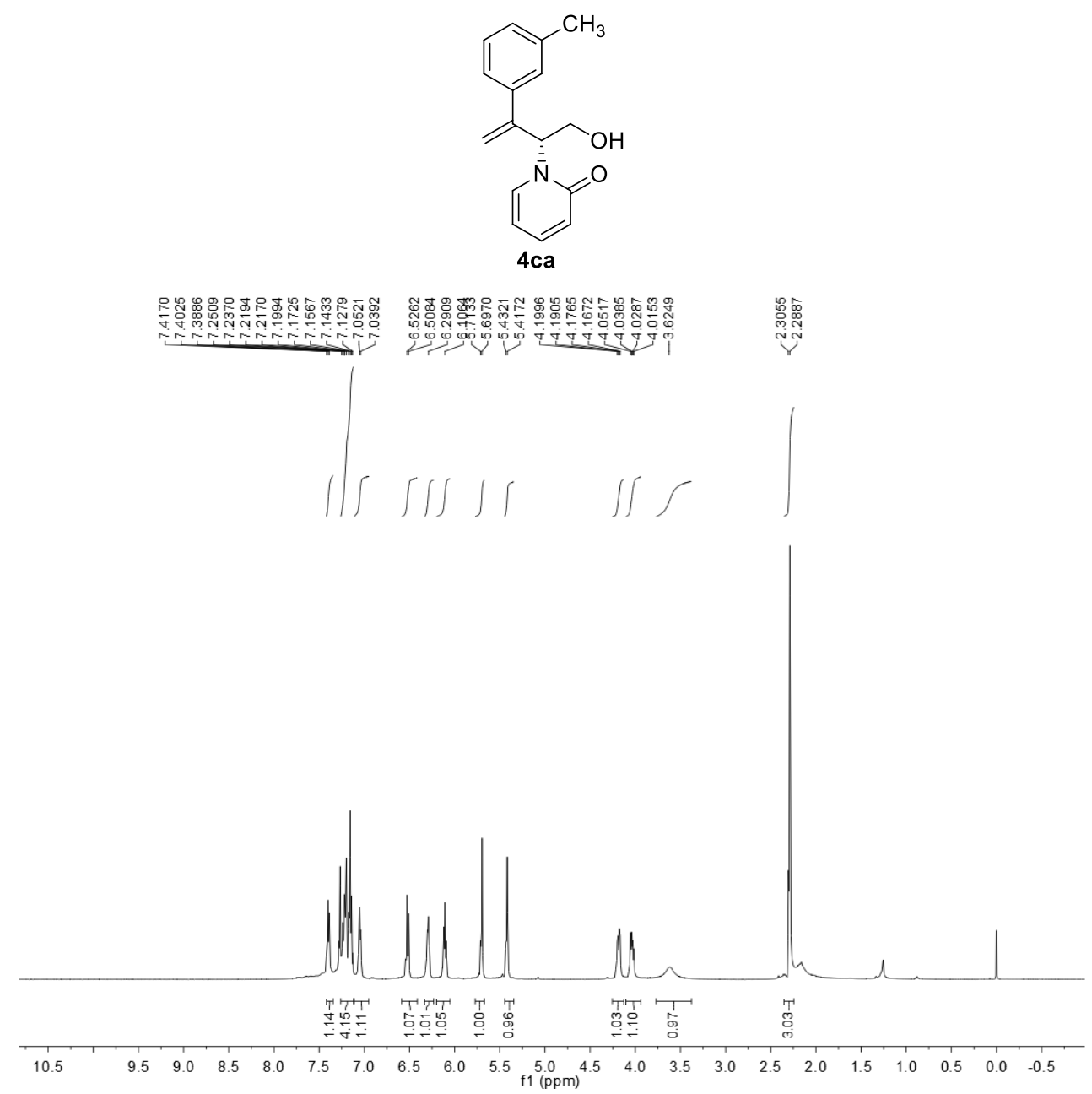

${ }^{1} \mathrm{H}$ NMR spectrum $\left(500 \mathrm{MHz}, \mathrm{CDCl}_{3}\right)$ of compound 4ca

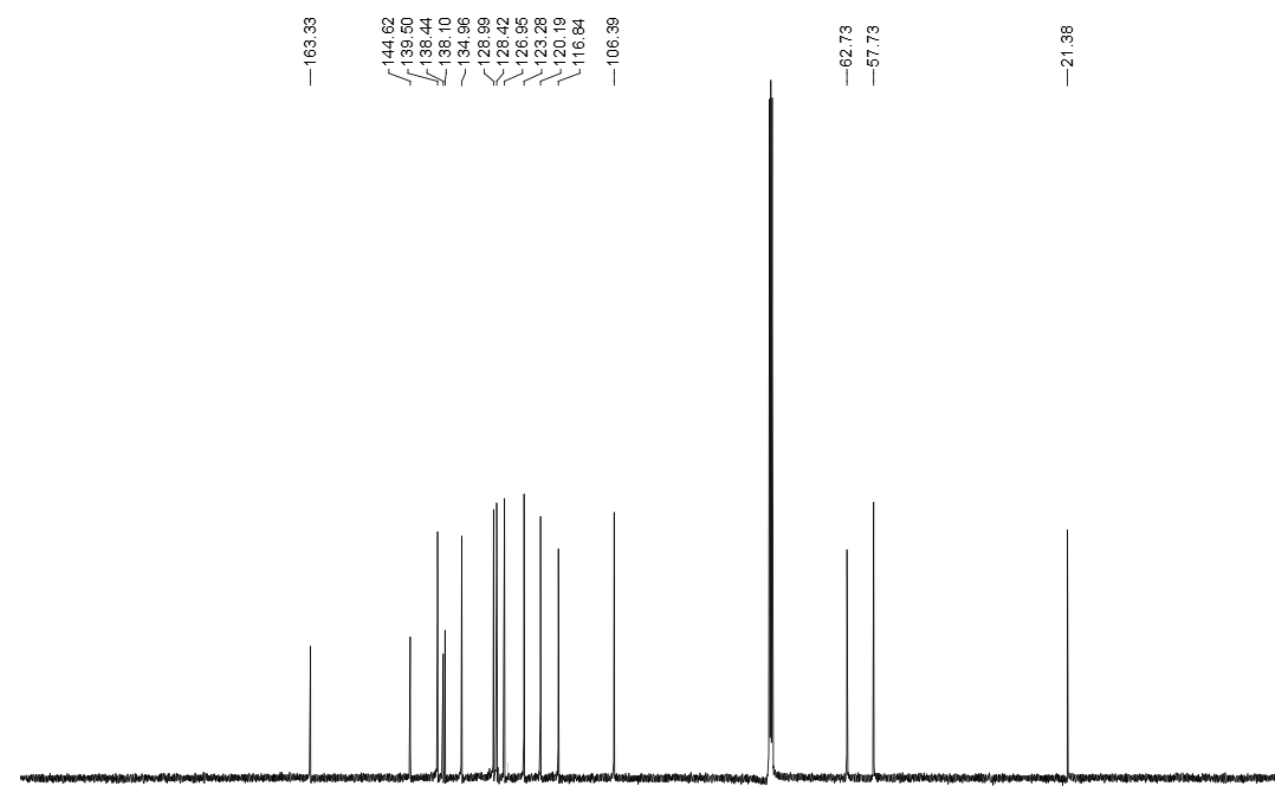

$\begin{array}{llllllllllllllllllllllllllll}210 & 200 & 190 & 180 & 170 & 160 & 150 & 140 & 130 & 120 & 110 & 100 & 90 & 80 & 70 & 60 & 50 & 40 & 30 & 20 & 10 & 0 & -10\end{array}$

${ }^{13} \mathrm{C}$ NMR spectrum $\left(125 \mathrm{MHz}, \mathrm{CDCl}_{3}\right)$ of compound $4 \mathbf{c a}$ 


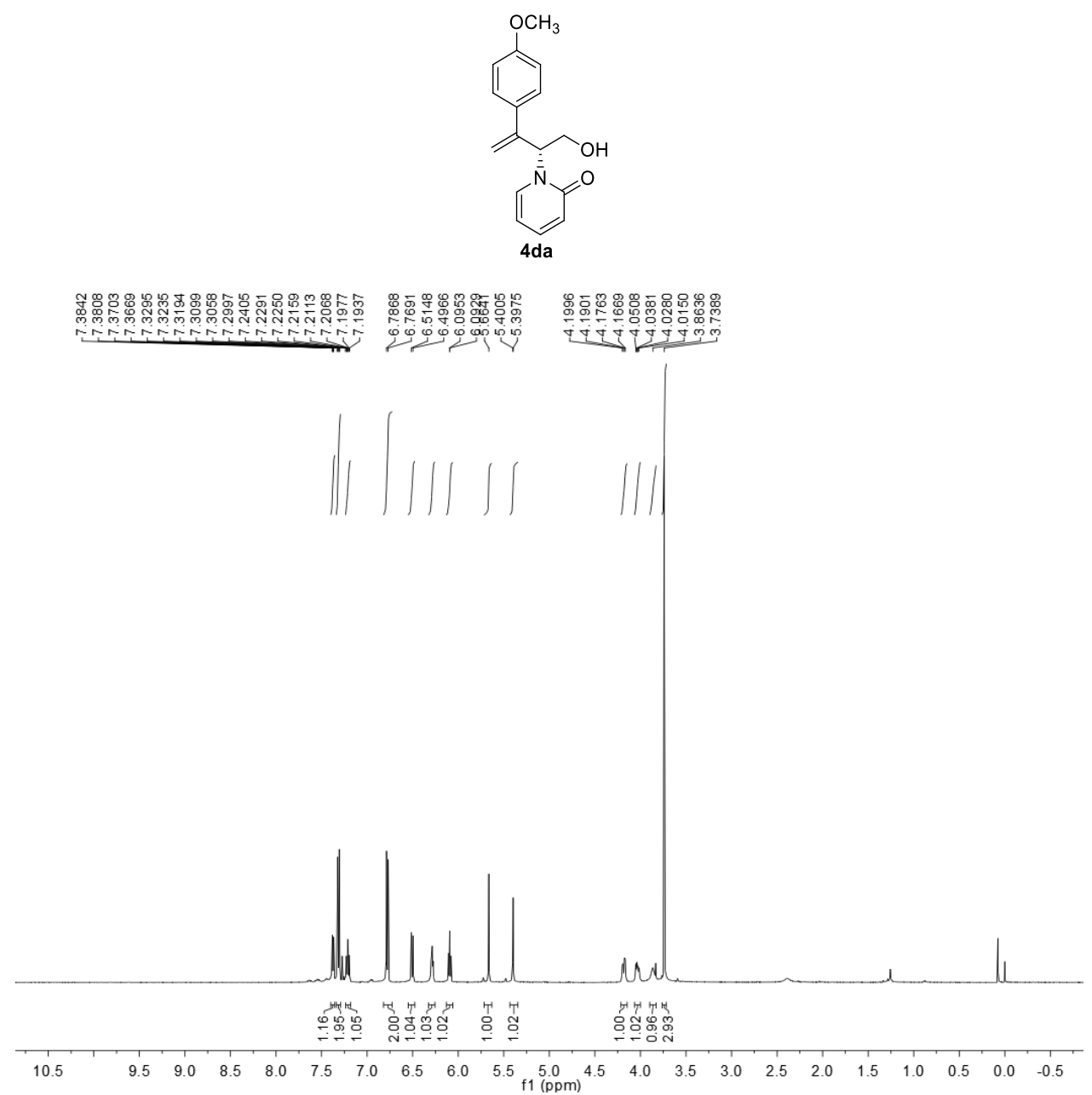

${ }^{1} \mathrm{H}$ NMR spectrum $\left(500 \mathrm{MHz}, \mathrm{CDCl}_{3}\right.$ ) of compound $\mathbf{4 d a}$

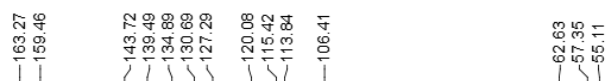

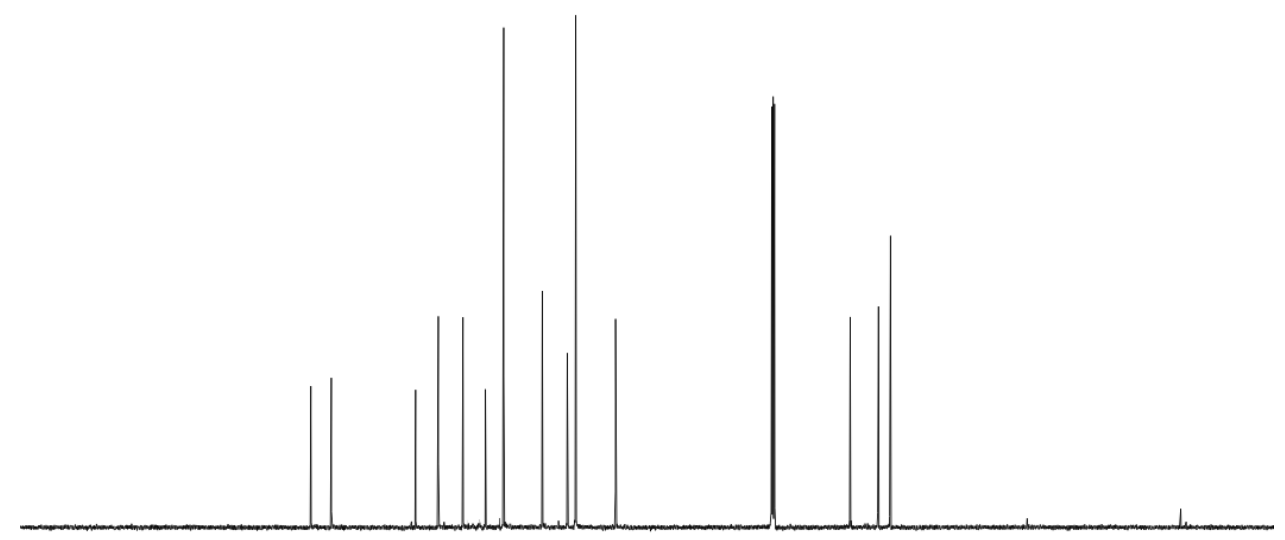

$\begin{array}{llllllllllllllllllllllllllll}210 & 200 & 190 & 180 & 170 & 160 & 150 & 140 & 130 & 120 & 110 & 100 & 90 & 80 & 70 & 60 & 50 & 40 & 30 & 20 & 10 & 0 & -10\end{array}$

${ }^{13} \mathrm{C}$ NMR spectrum $\left(125 \mathrm{MHz}, \mathrm{CDCl}_{3}\right)$ of compound $4 \mathbf{d a}$ 

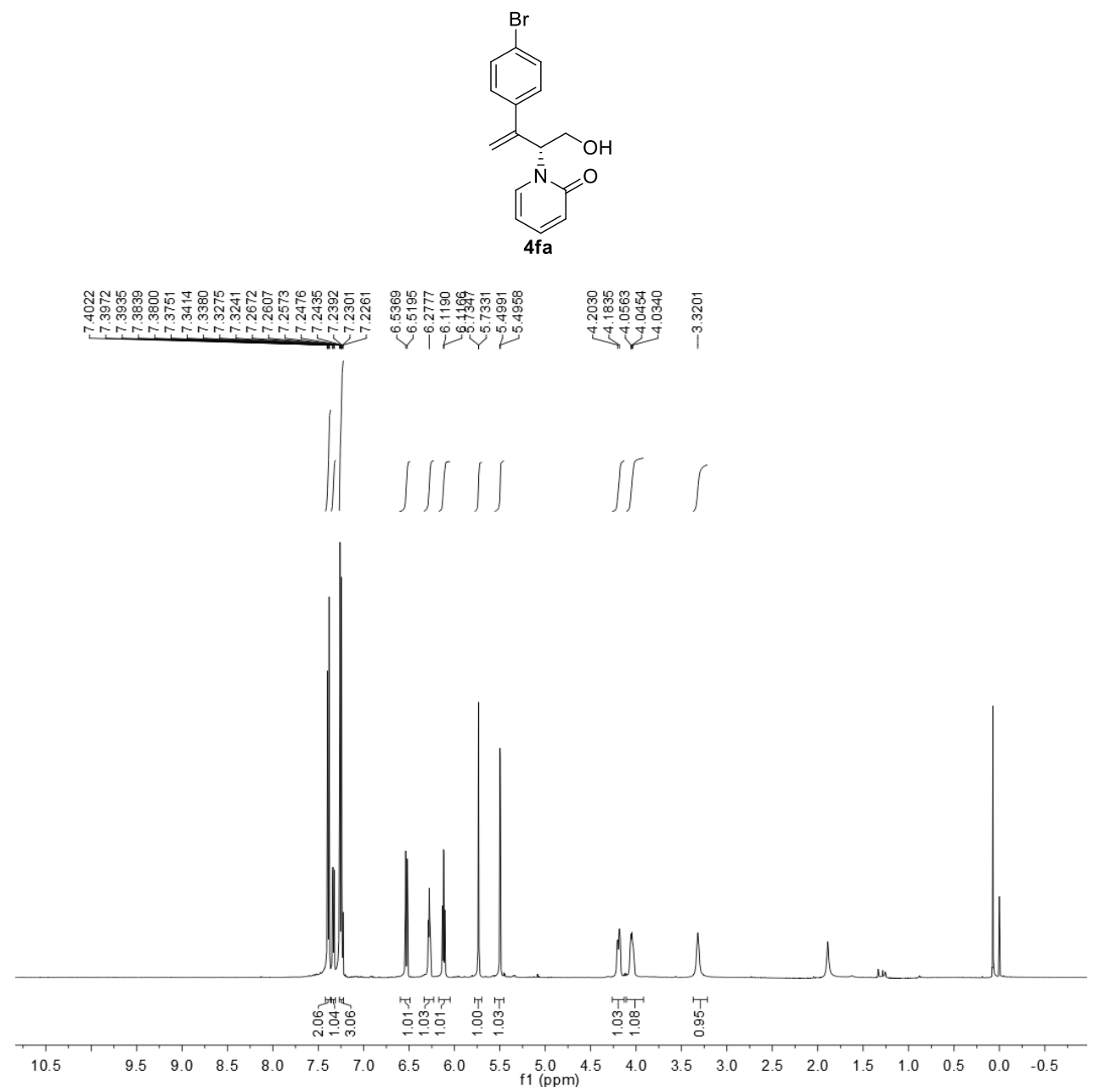

${ }^{1} \mathrm{H}$ NMR spectrum $\left(500 \mathrm{MHz}, \mathrm{CDCl}_{3}\right)$ of compound $4 \mathbf{f a}$

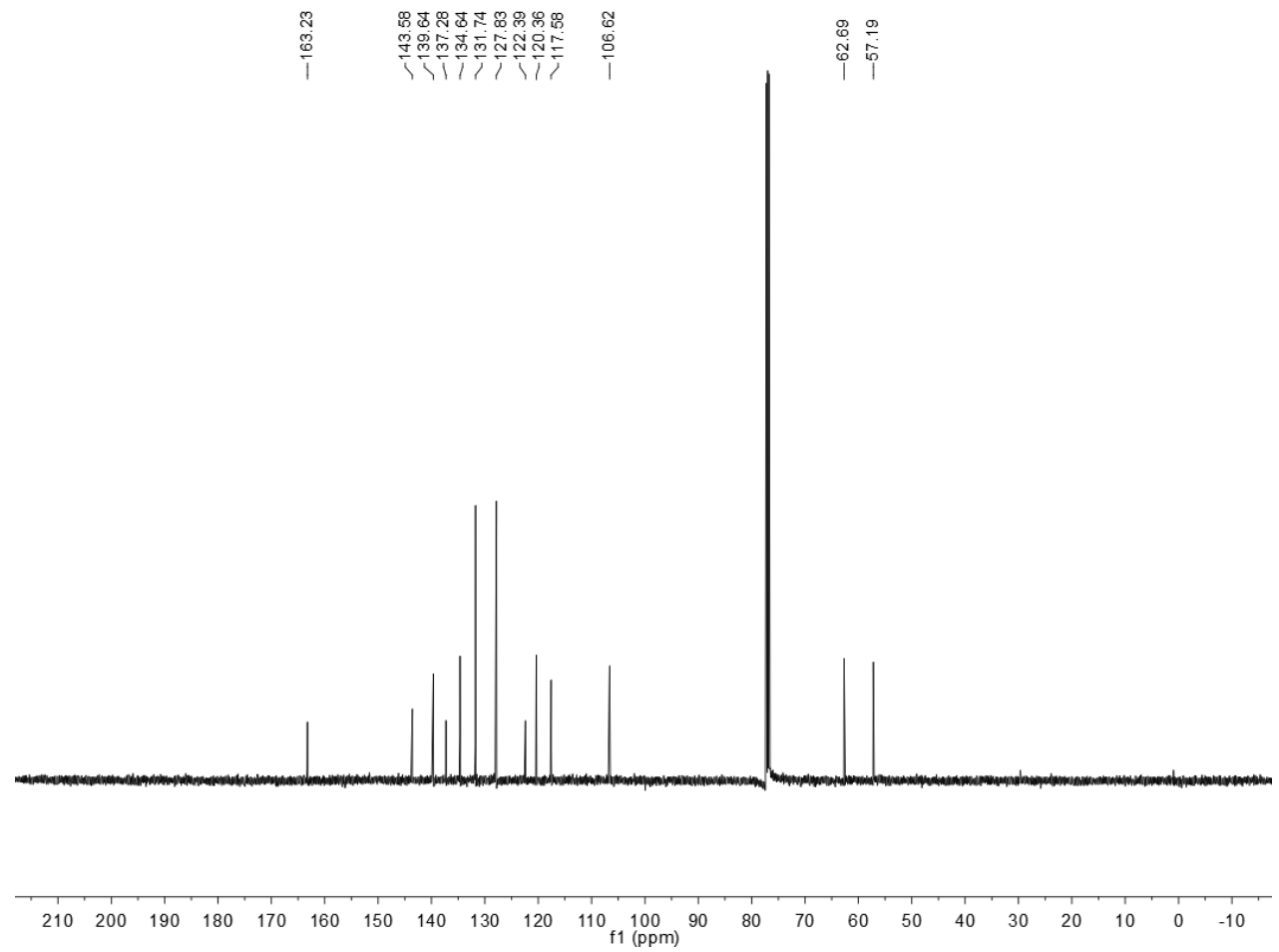

${ }^{13} \mathrm{C}$ NMR spectrum (125 MHz, $\mathrm{CDCl}_{3}$ ) of compound $\mathbf{4 f a}$ 


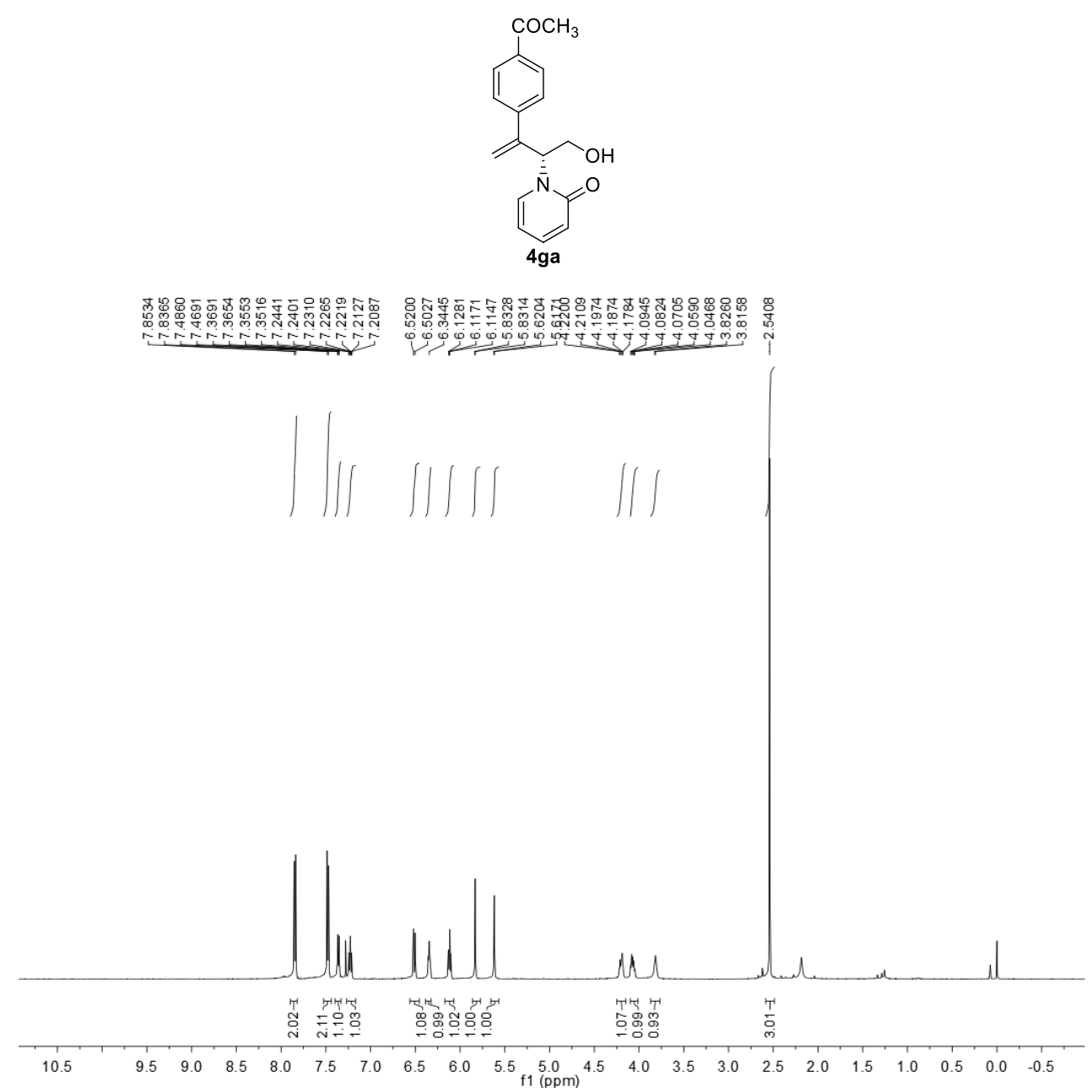

${ }^{1} \mathrm{H}$ NMR spectrum $\left(500 \mathrm{MHz}, \mathrm{CDCl}_{3}\right)$ of compound $\mathbf{4 g a}$

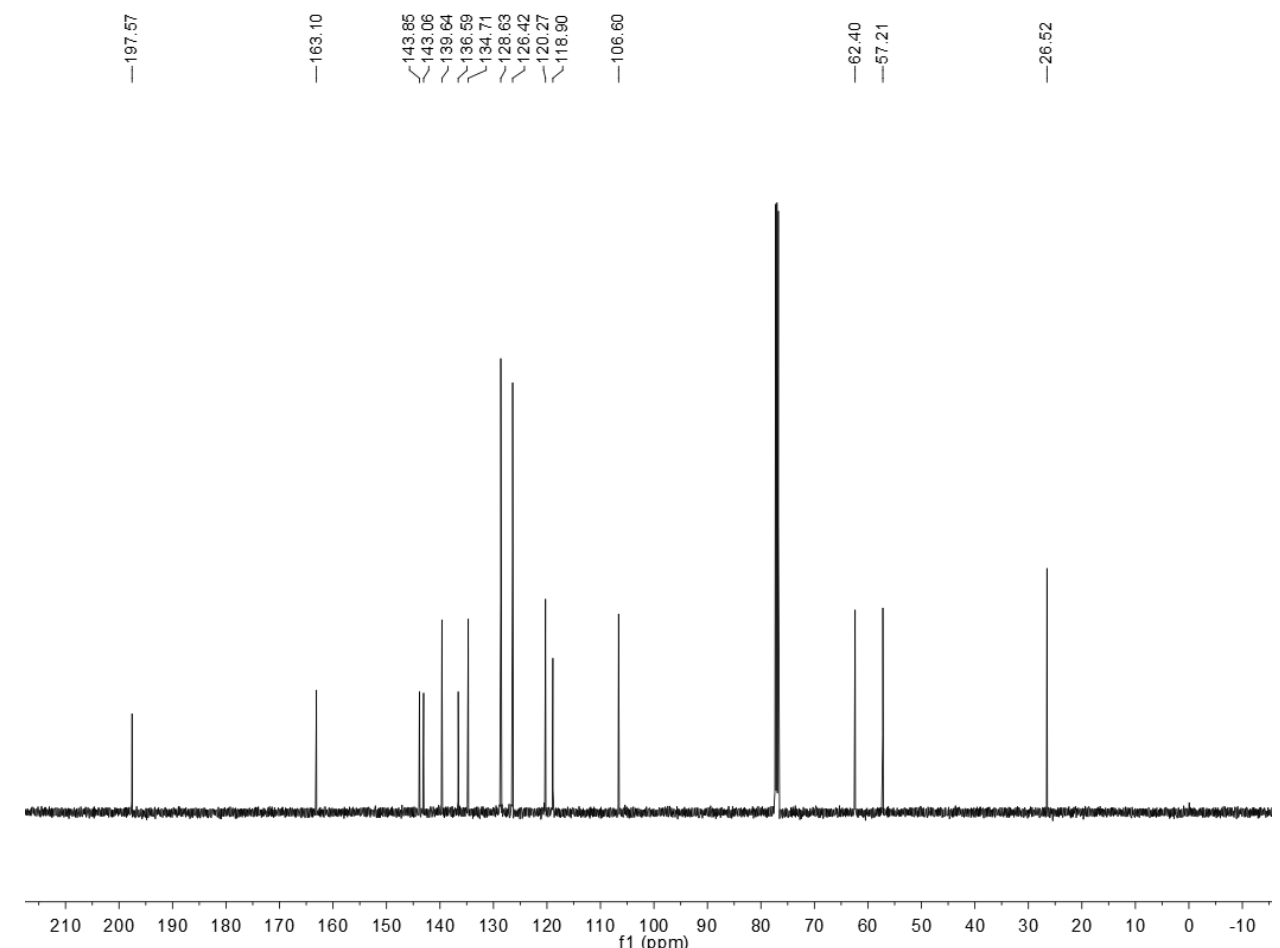

${ }^{13} \mathrm{C}$ NMR spectrum (125 MHz, $\mathrm{CDCl}_{3}$ ) of compound 4ga 


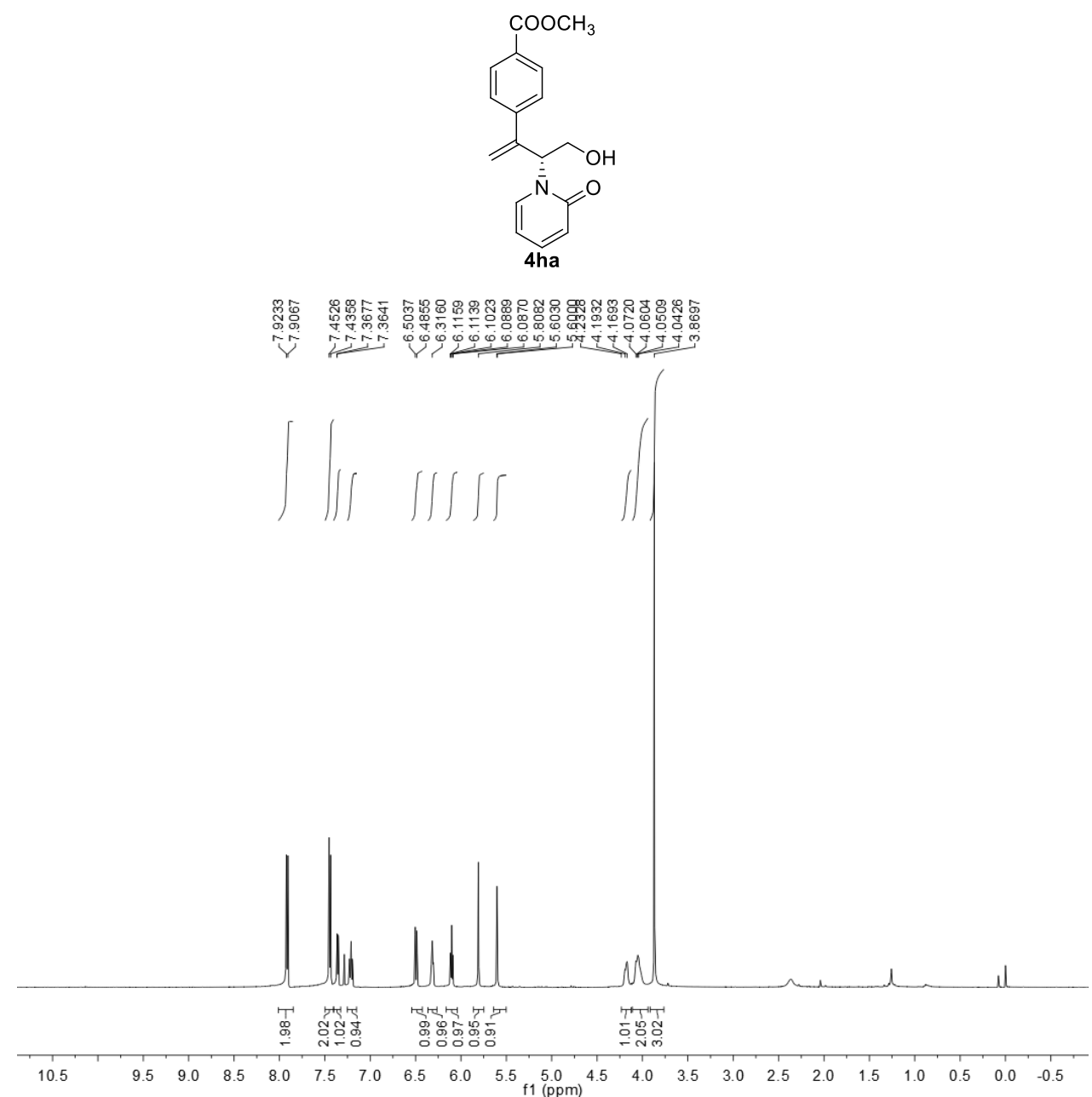

${ }^{1} \mathrm{H}$ NMR spectrum $\left(500 \mathrm{MHz}, \mathrm{CDCl}_{3}\right)$ of compound 4 ha

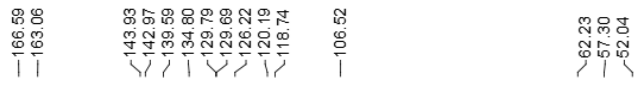

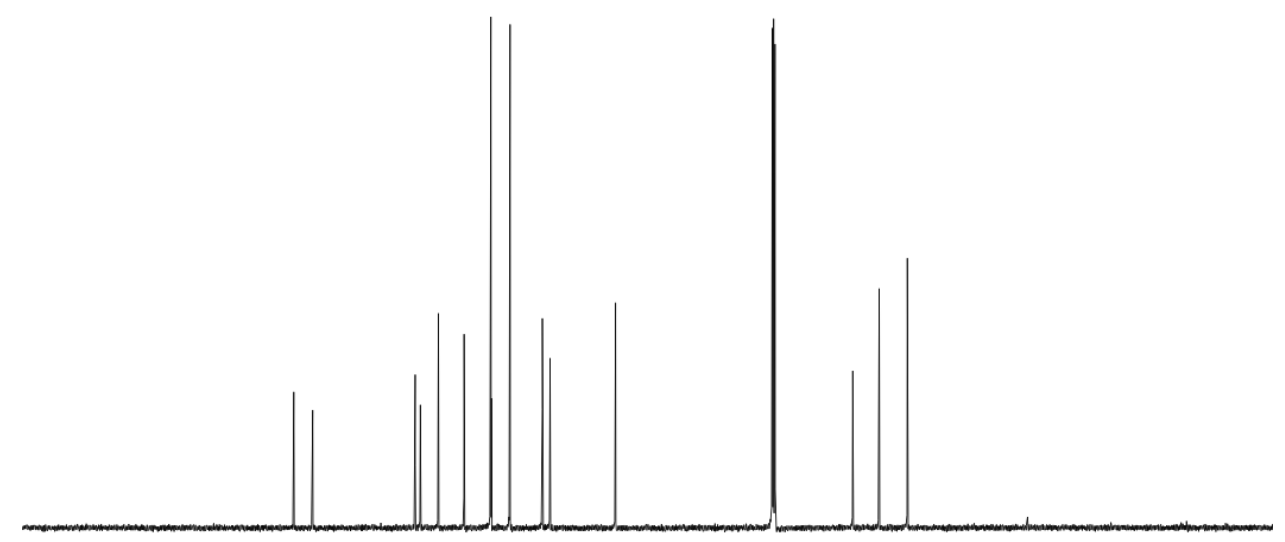

$\begin{array}{llllllllllllllllllllllllll}210 & 200 & 190 & 180 & 170 & 160 & 150 & 140 & 130 & 120 & 110 & 100 & 90 & 80 & 70 & 60 & 50 & 40 & 30 & 20 & 10 & 0 & -10\end{array}$

${ }^{13} \mathrm{C}$ NMR spectrum $\left(125 \mathrm{MHz}, \mathrm{CDCl}_{3}\right)$ of compound 4 ha 

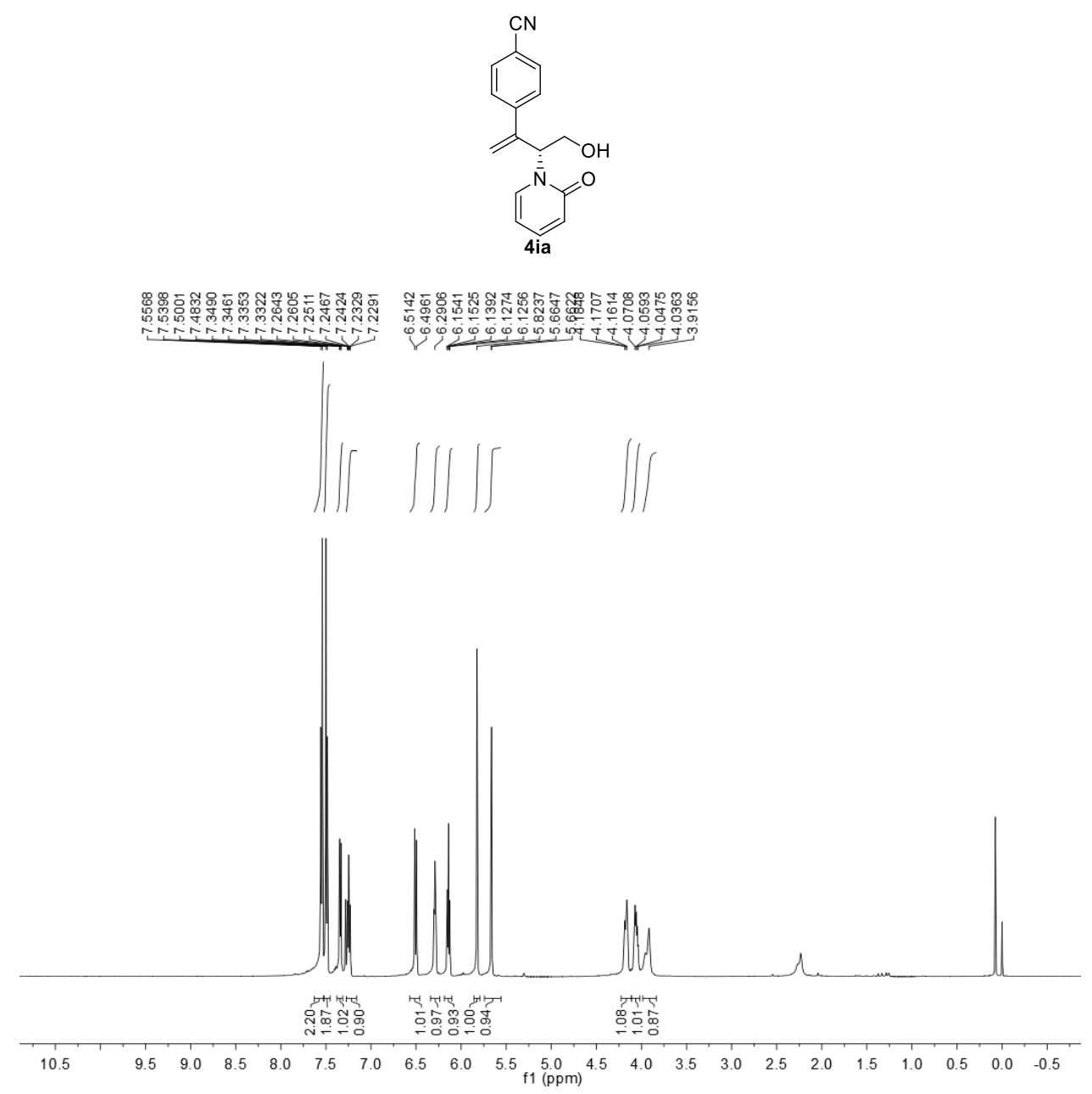

${ }^{1} \mathrm{H}$ NMR spectrum $\left(500 \mathrm{MHz}, \mathrm{CDCl}_{3}\right)$ of compound 4ia

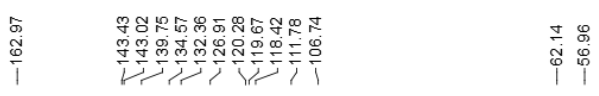

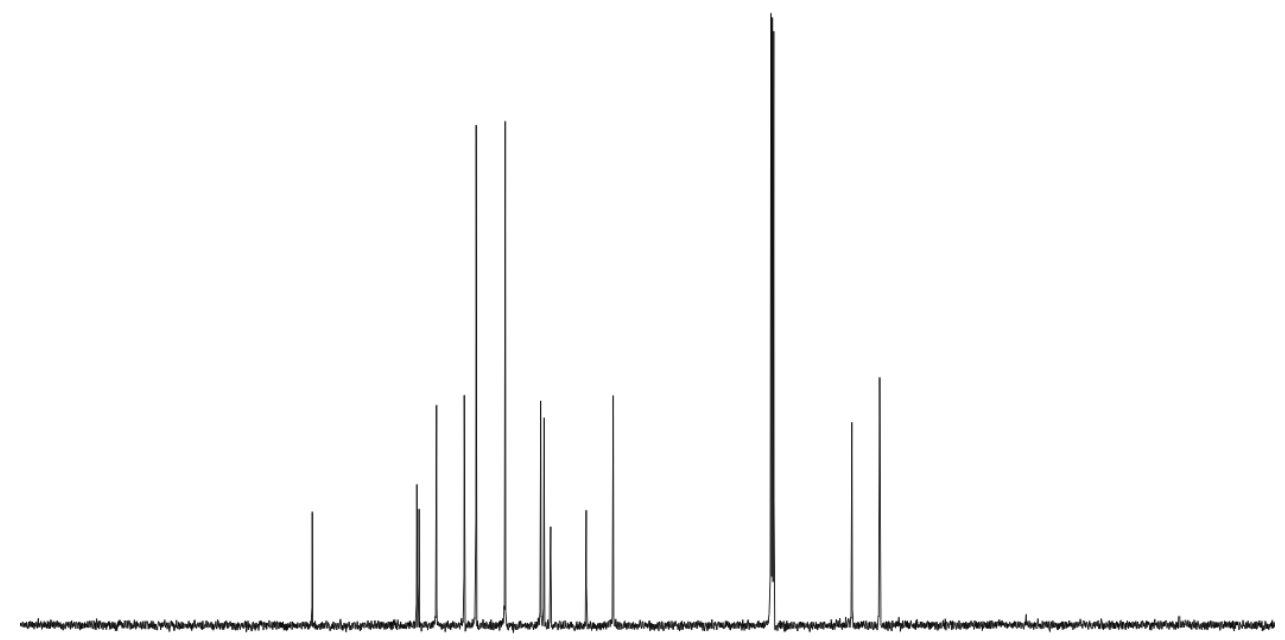

$\begin{array}{llllllllllllllllllllllllll}210 & 200 & 190 & 180 & 170 & 160 & 150 & 140 & 130 & 120 & 110 \begin{array}{l}100 \\ \mathrm{f} 1(\mathrm{ppm})\end{array} & 90 & 80 & 70 & 60 & 50 & 40 & 30 & 20 & 10 & 0 & -10\end{array}$

${ }^{13} \mathrm{C}$ NMR spectrum $\left(125 \mathrm{MHz}, \mathrm{CDCl}_{3}\right)$ of compound $4 \mathbf{i a}$ 

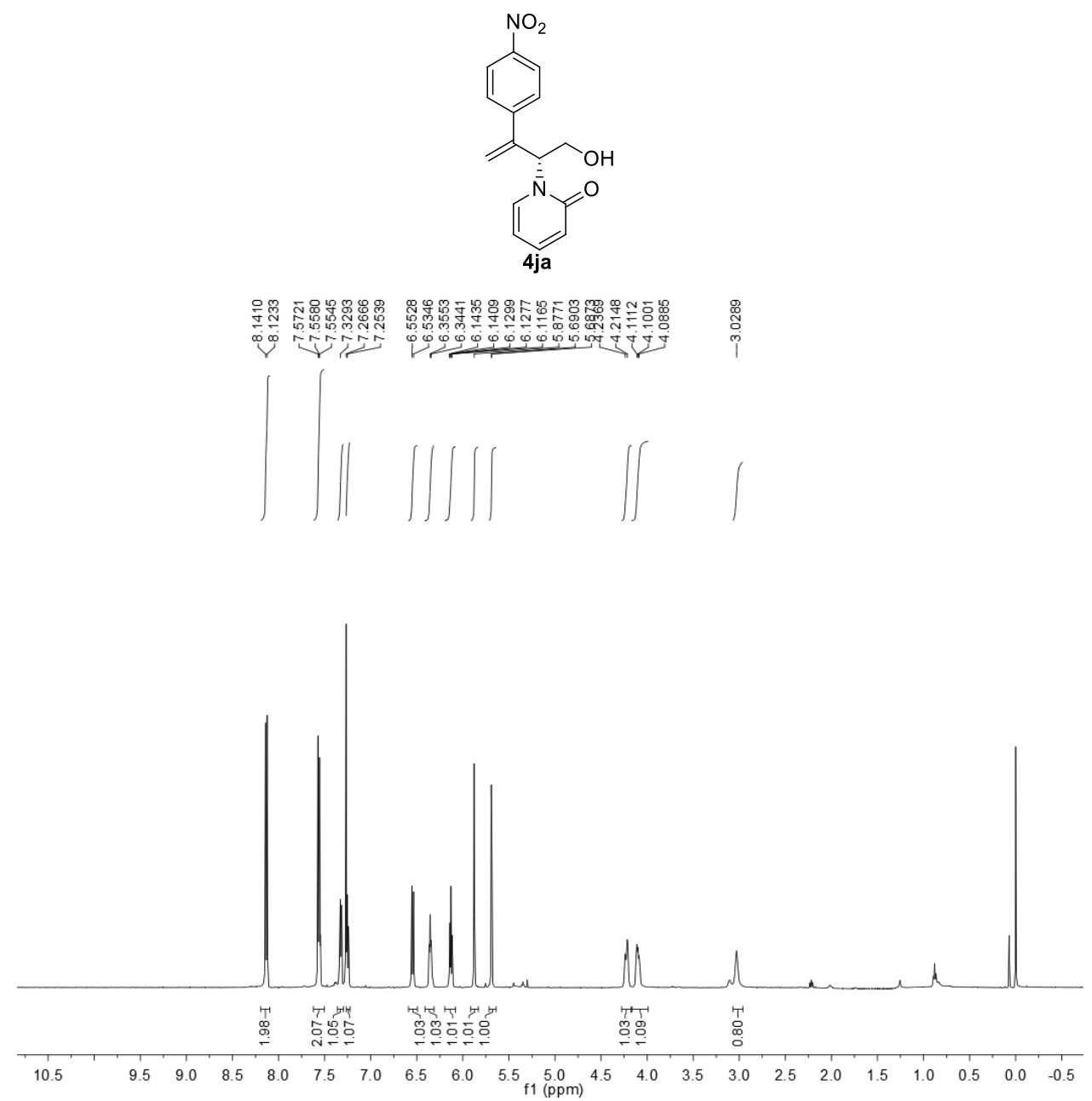

${ }^{1} \mathrm{H}$ NMR spectrum $\left(500 \mathrm{MHz}, \mathrm{CDCl}_{3}\right)$ of compound $\mathbf{4 j a}$

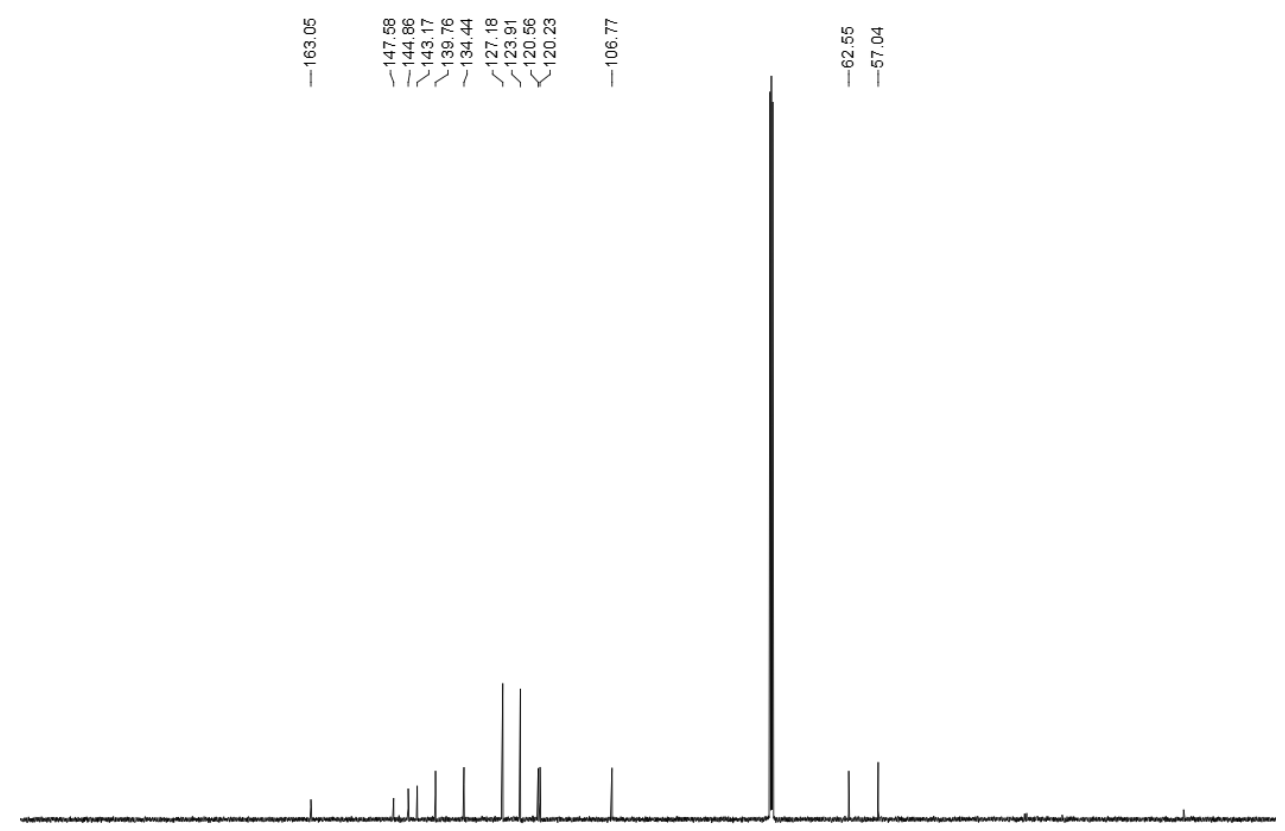

$\begin{array}{lllllllllllllllllllllllll}210 & 200 & 190 & 180 & 170 & 160 & 150 & 140 & 130 & 120 & 110 & 100 & 90 & 80 & 70 & 60 & 50 & 40 & 30 & 20 & 10 & 0 & -10\end{array}$

${ }^{13} \mathrm{C}$ NMR spectrum $\left(125 \mathrm{MHz}, \mathrm{CDCl}_{3}\right)$ of compound $\mathbf{4 j a}$ 

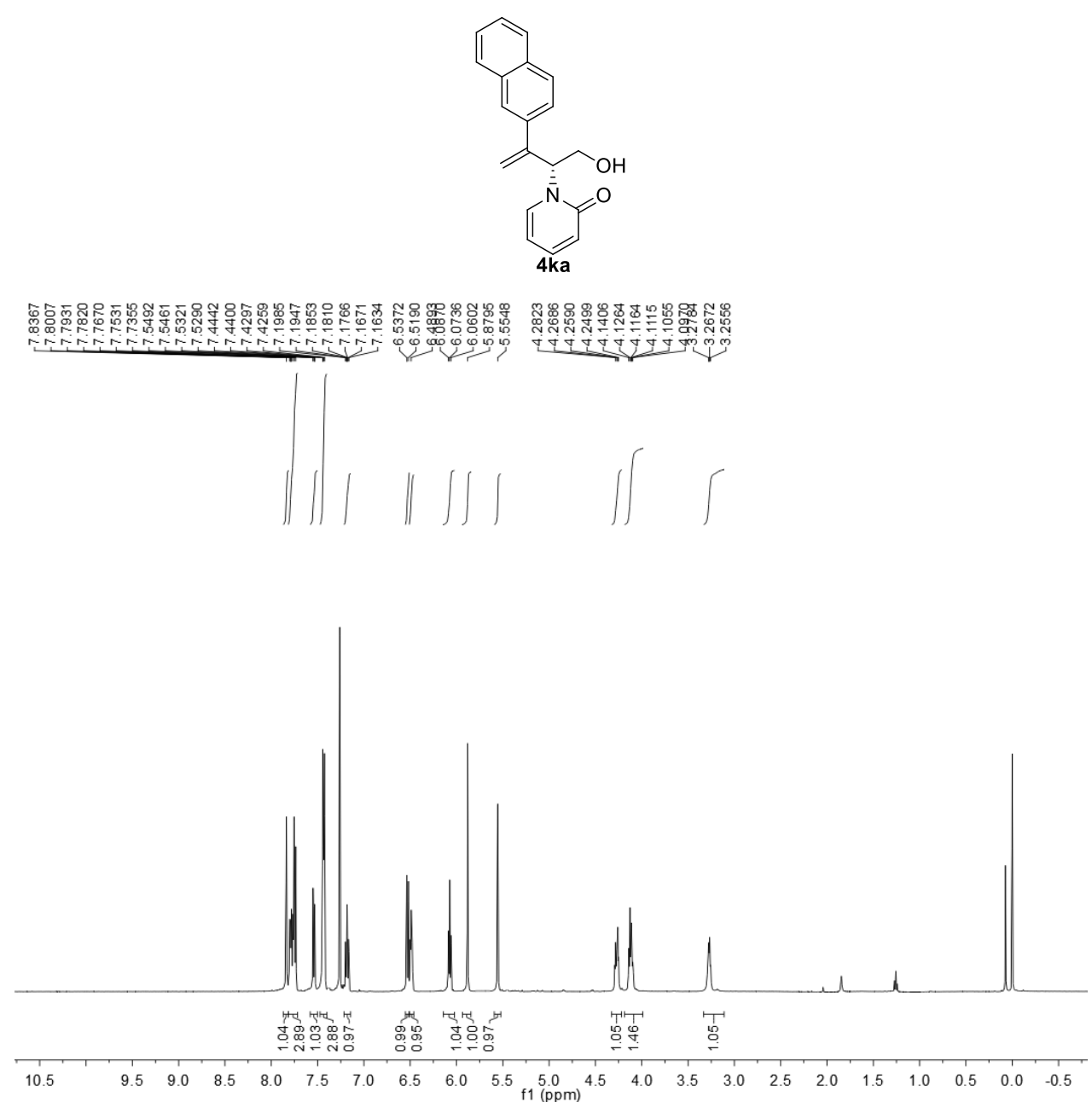

${ }^{1} \mathrm{H}$ NMR spectrum $\left(500 \mathrm{MHz}, \mathrm{CDCl}_{3}\right)$ of compound $4 \mathbf{k a}$

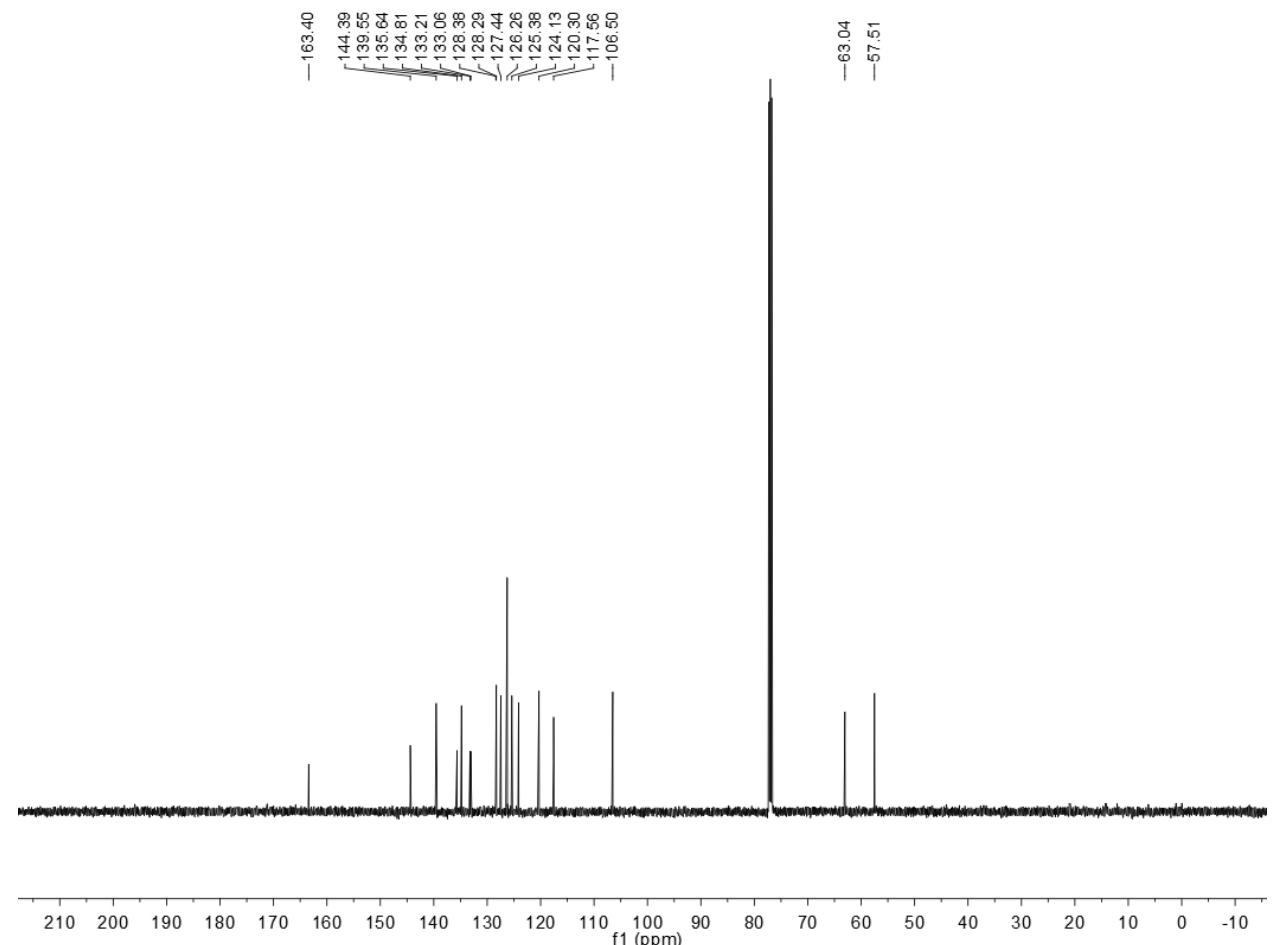

${ }^{13} \mathrm{C}$ NMR spectrum $\left(125 \mathrm{MHz}, \mathrm{CDCl}_{3}\right)$ of compound $4 \mathbf{k a}$ 

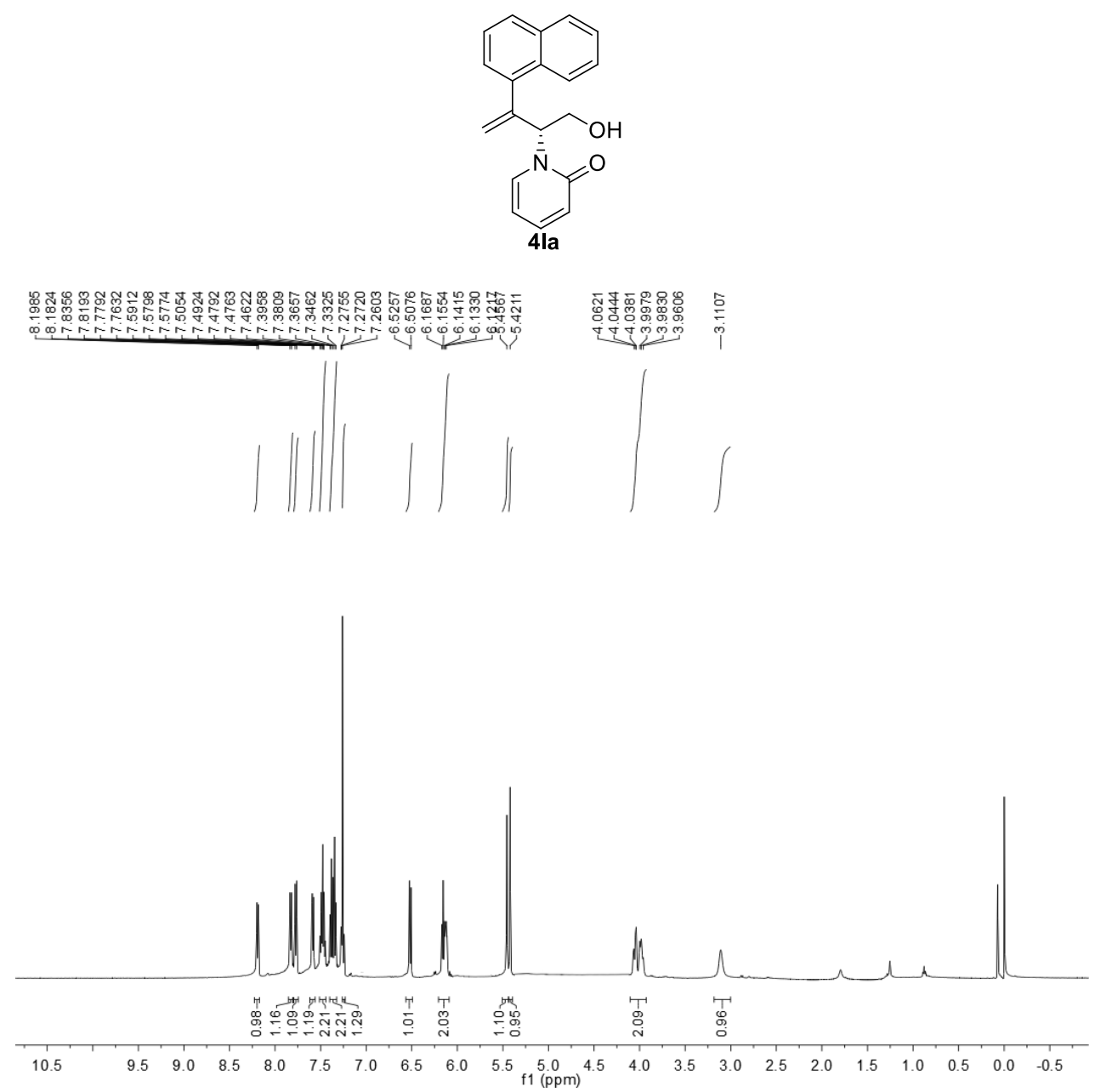

${ }^{1} \mathrm{H}$ NMR spectrum $\left(500 \mathrm{MHz}, \mathrm{CDCl}_{3}\right)$ of compound 4la

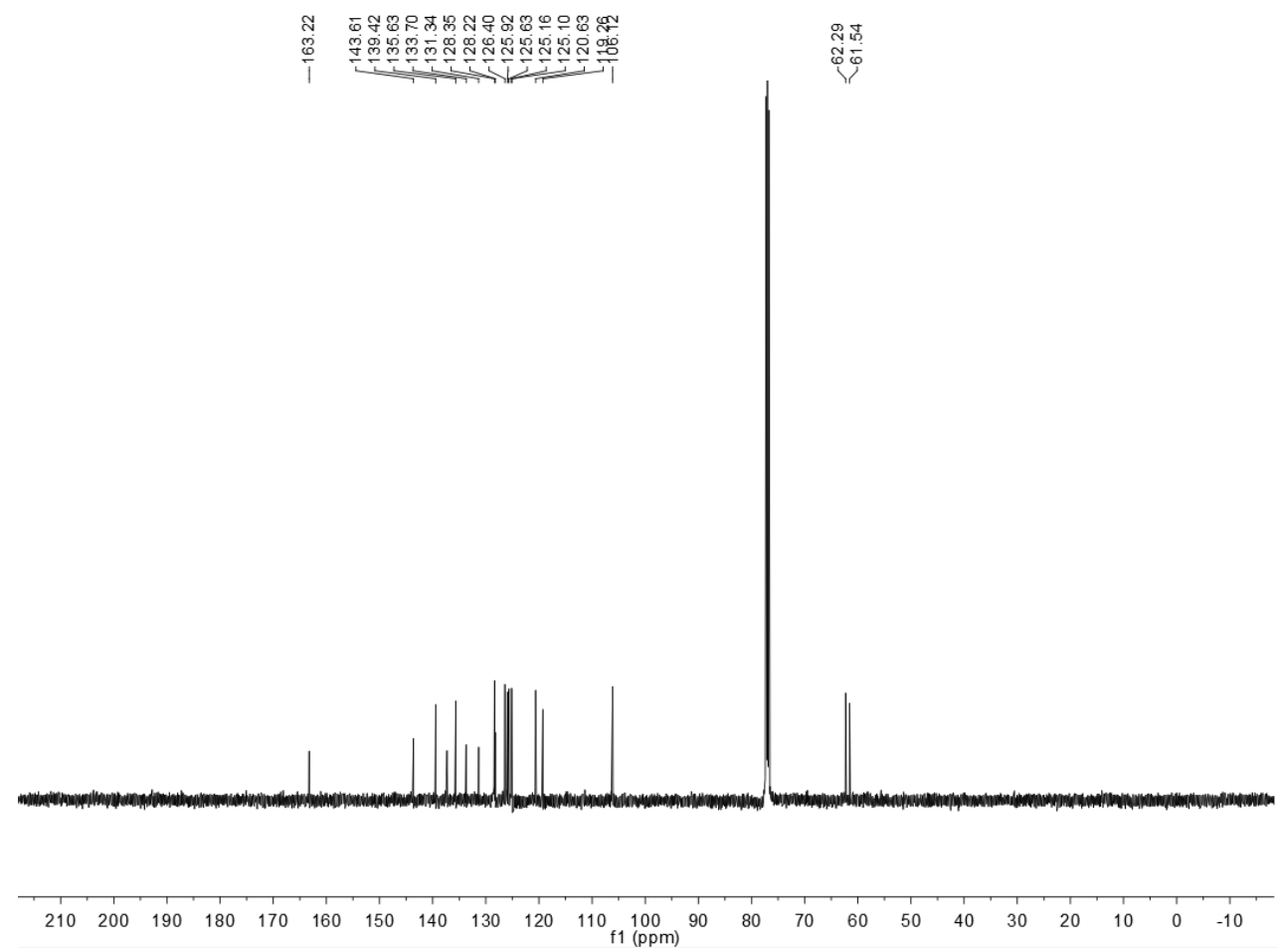

${ }^{13} \mathrm{C}$ NMR spectrum $\left(125 \mathrm{MHz}, \mathrm{CDCl}_{3}\right)$ of compound $4 \mathbf{l a}$ 
<smiles>C=C(c1ccccc1)C(CO)n1cc(C)ccc1=O</smiles>

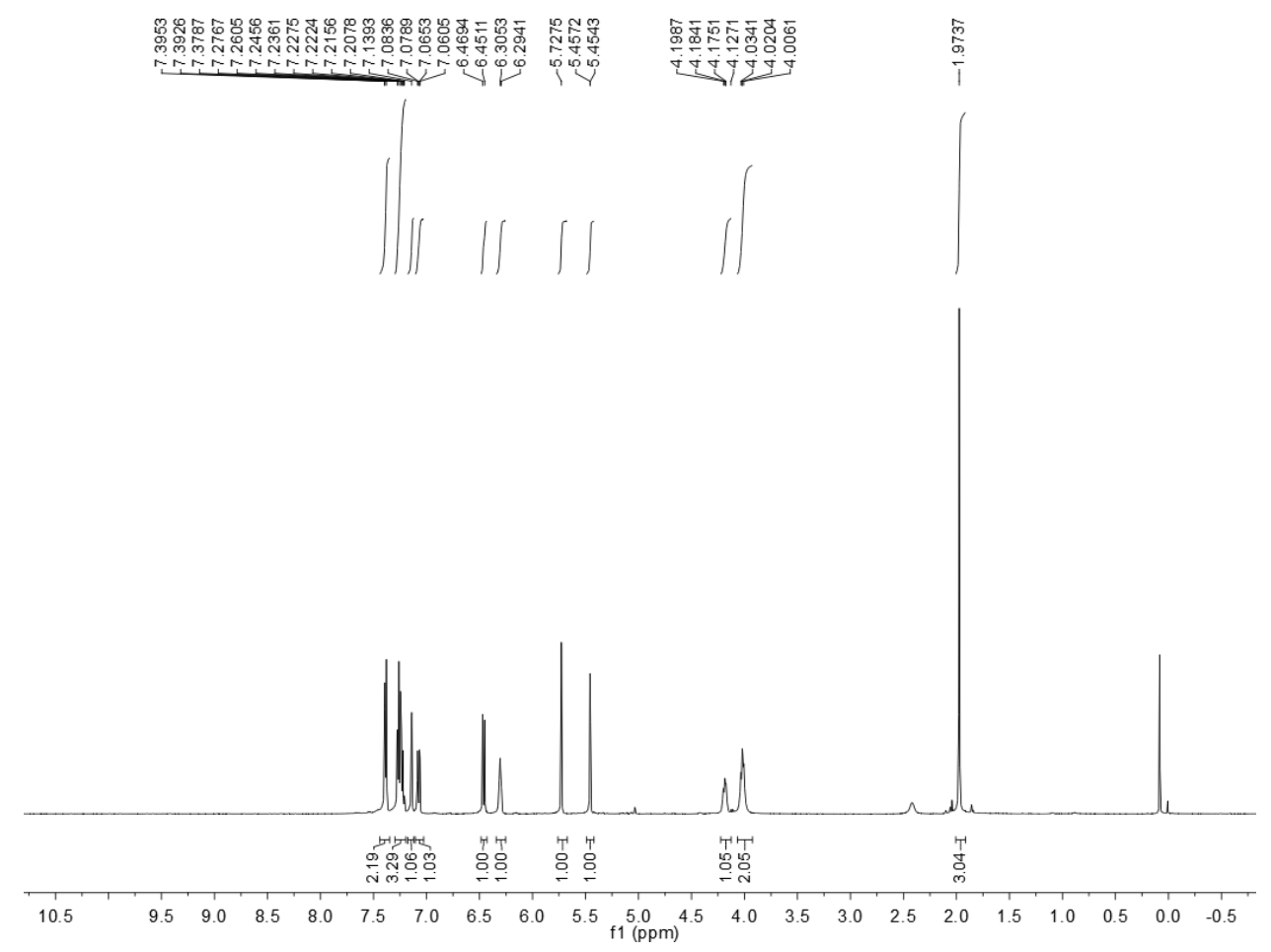

${ }^{1} \mathrm{H}$ NMR spectrum $\left(500 \mathrm{MHz}, \mathrm{CDCl}_{3}\right)$ of compound $\mathbf{4 a b}$
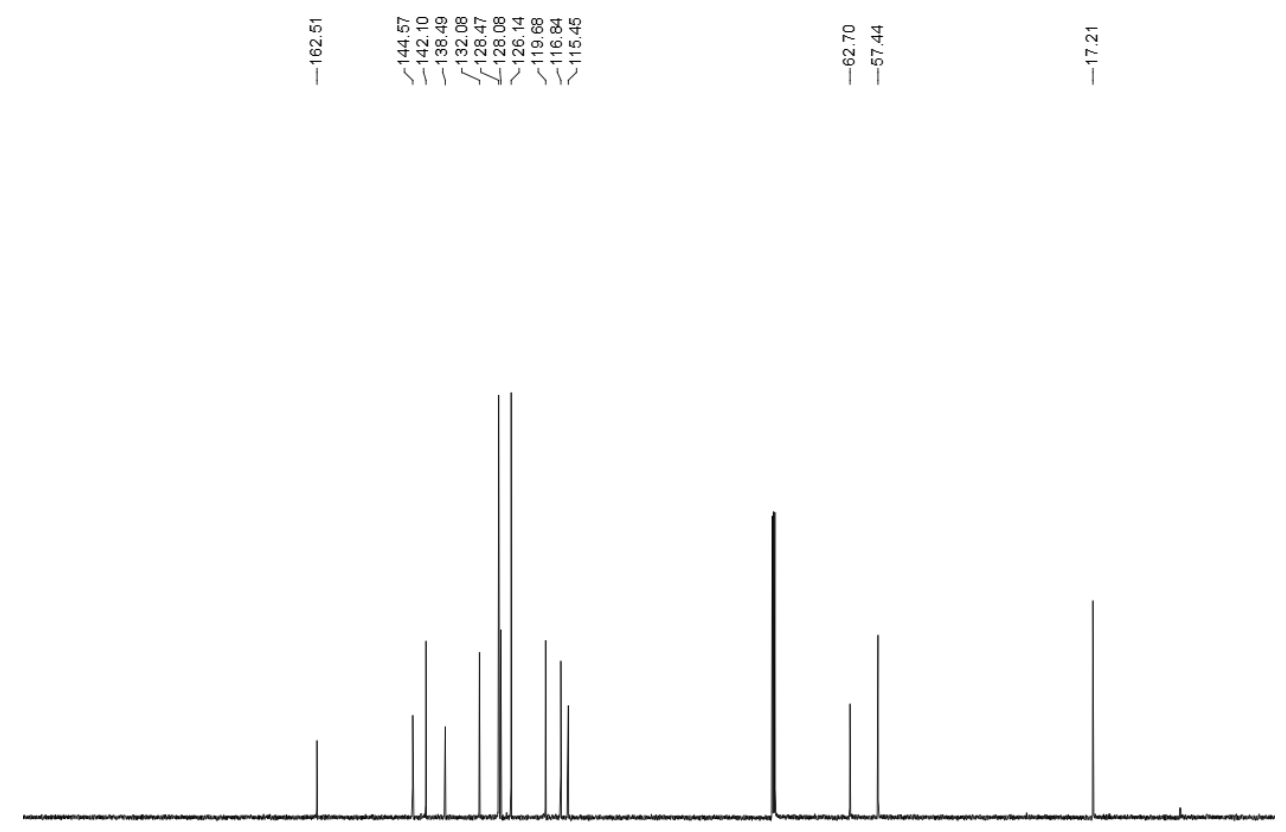

$\begin{array}{lllllllllllllllllllllllllll}210 & 200 & 190 & 180 & 170 & 160 & 150 & 140 & 130 & 120 & 110 & 100 & 90 & 80 & 70 & 60 & 50 & 40 & 30 & 20 & 10 & 0 & -10\end{array}$

${ }^{13} \mathrm{C}$ NMR spectrum $\left(125 \mathrm{MHz}, \mathrm{CDCl}_{3}\right)$ of compound $\mathbf{4 a b}$ 

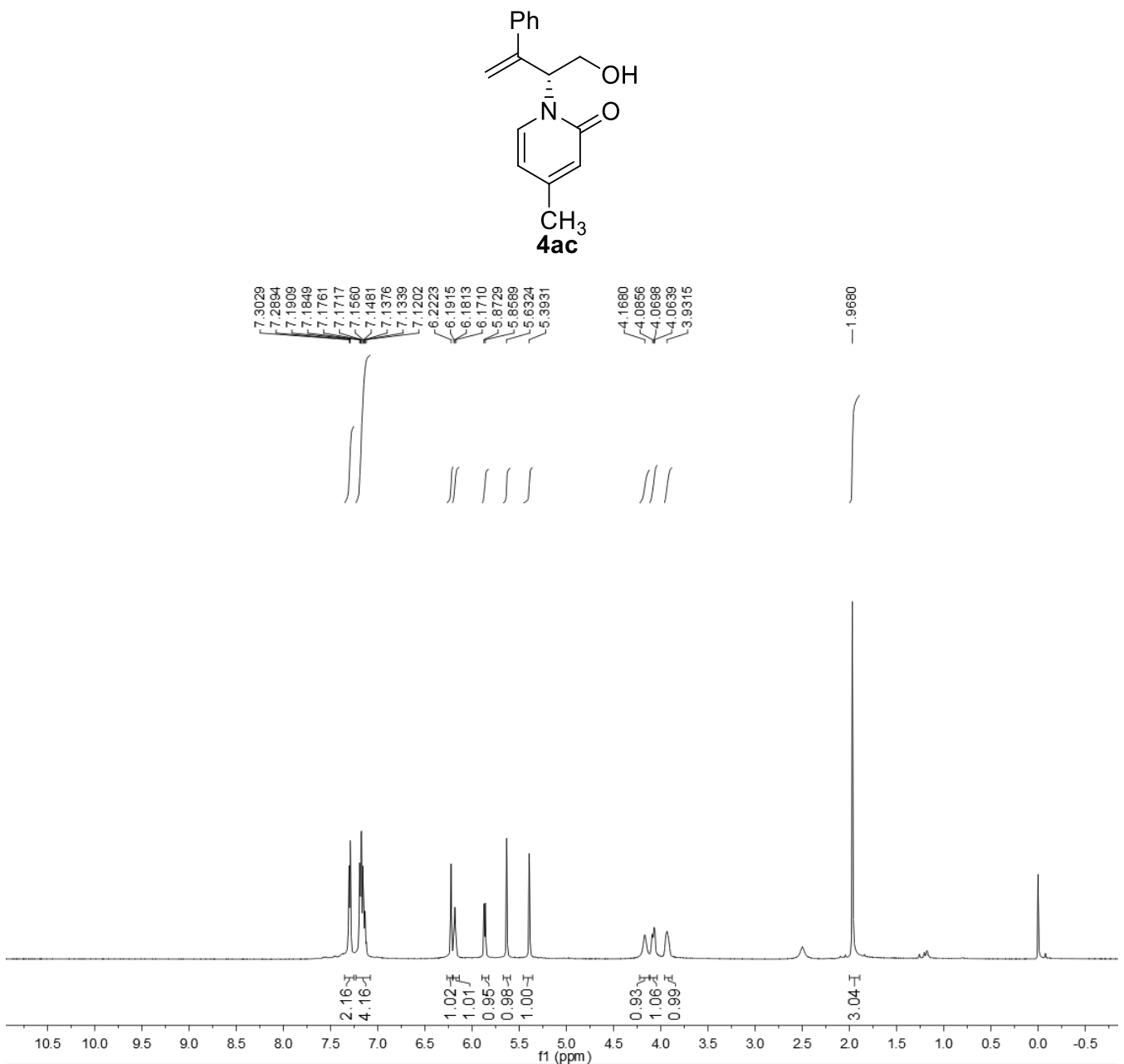

${ }^{1} \mathrm{H}$ NMR spectrum $\left(500 \mathrm{MHz}, \mathrm{CDCl}_{3}\right)$ of compound $4 \mathbf{a c}$

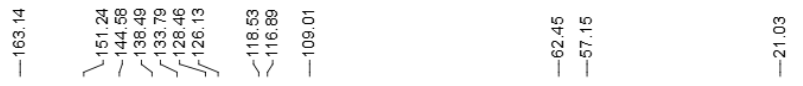

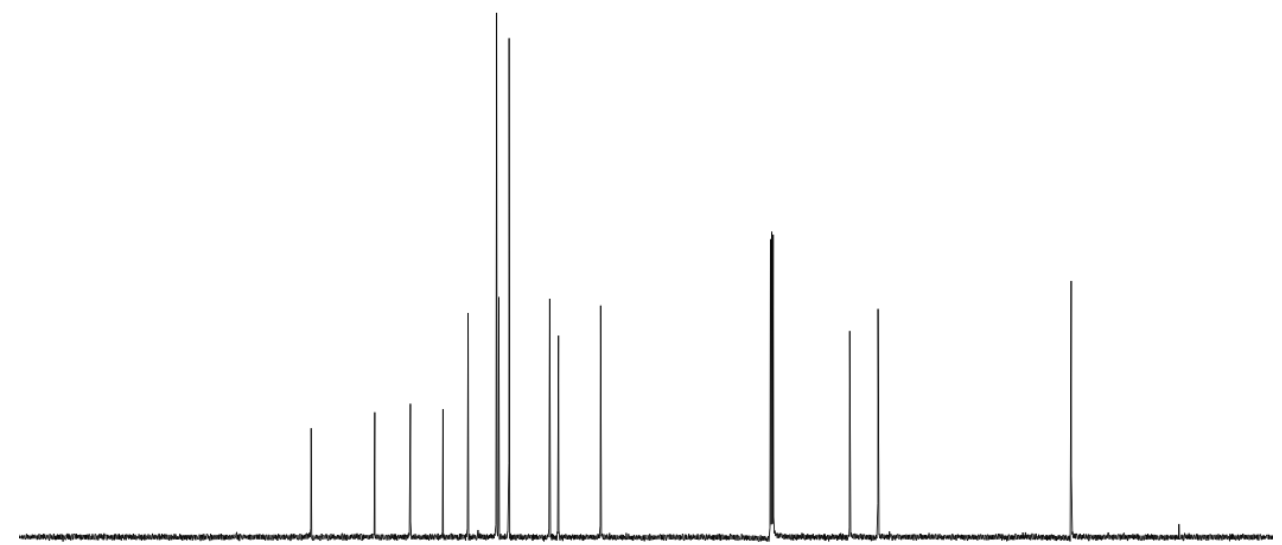

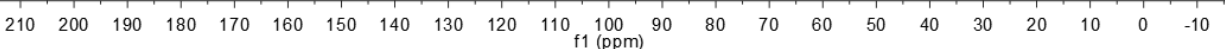

${ }^{13} \mathrm{C}$ NMR spectrum $\left(125 \mathrm{MHz}, \mathrm{CDCl}_{3}\right)$ of compound $4 \mathbf{a c}$ 
<smiles>C=C(c1ccccc1)[C@H](CO)n1ccc([14CH3])c(C)c1=O</smiles>

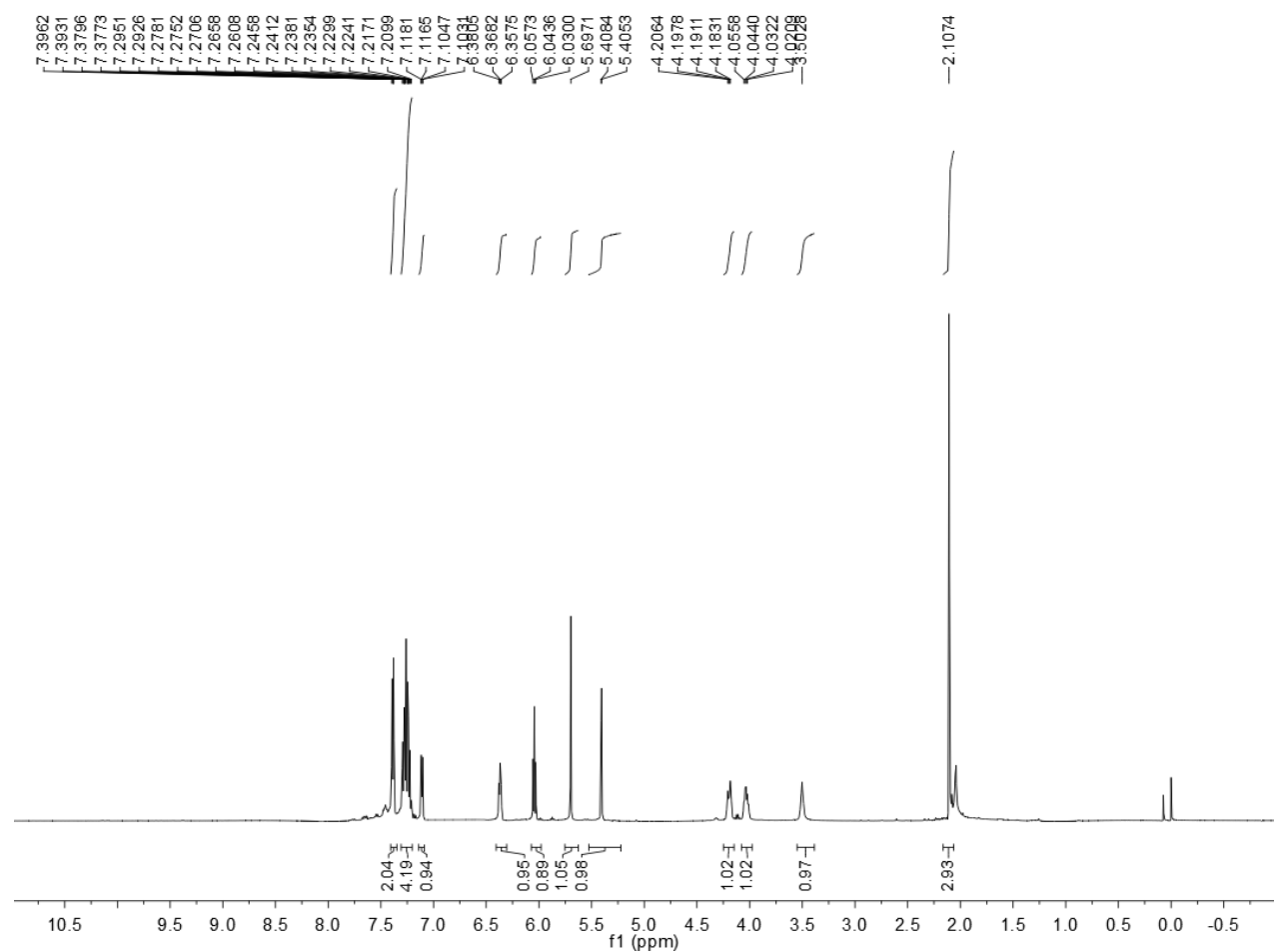

${ }^{1} \mathrm{H}$ NMR spectrum $\left(500 \mathrm{MHz}, \mathrm{CDCl}_{3}\right)$ of compound $\mathbf{4 a d}$

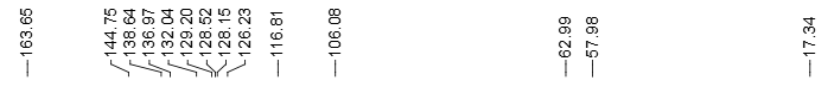

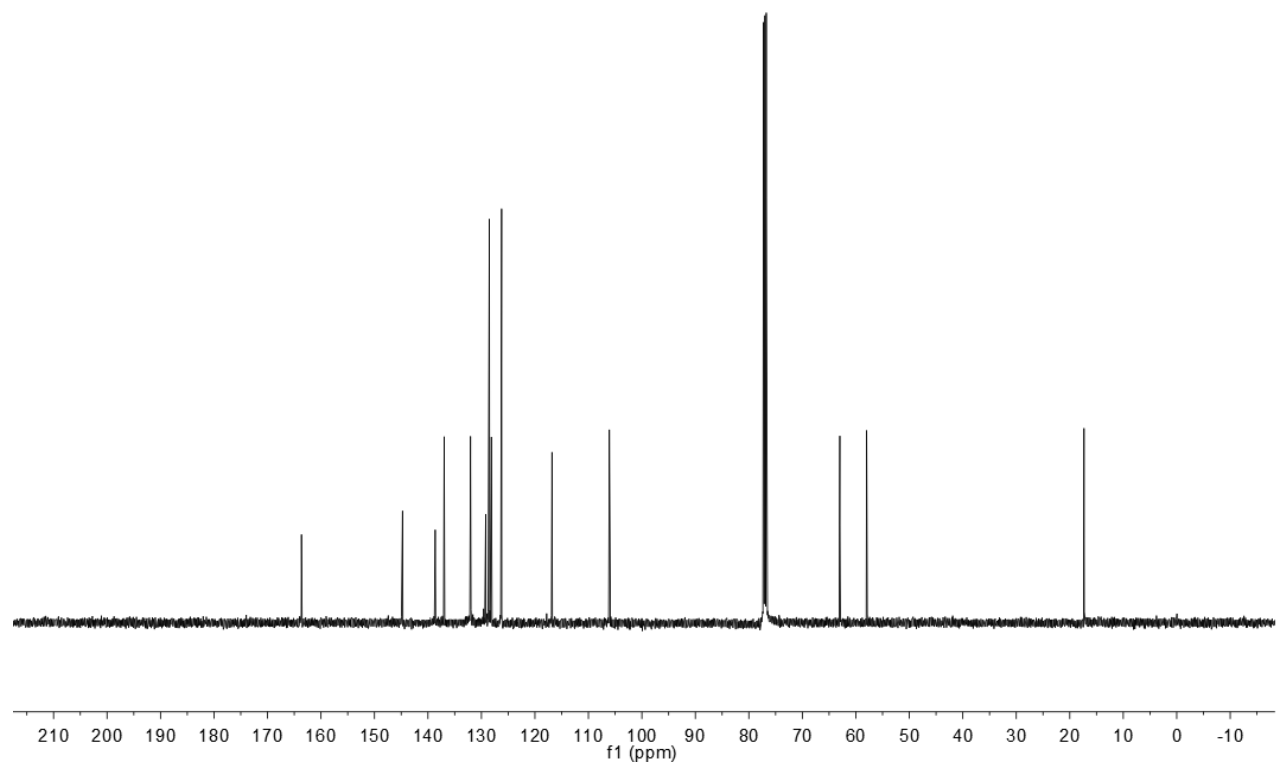

${ }^{13} \mathrm{C}$ NMR spectrum $\left(125 \mathrm{MHz}, \mathrm{CDCl}_{3}\right)$ of compound $\mathbf{4 a d}$ 

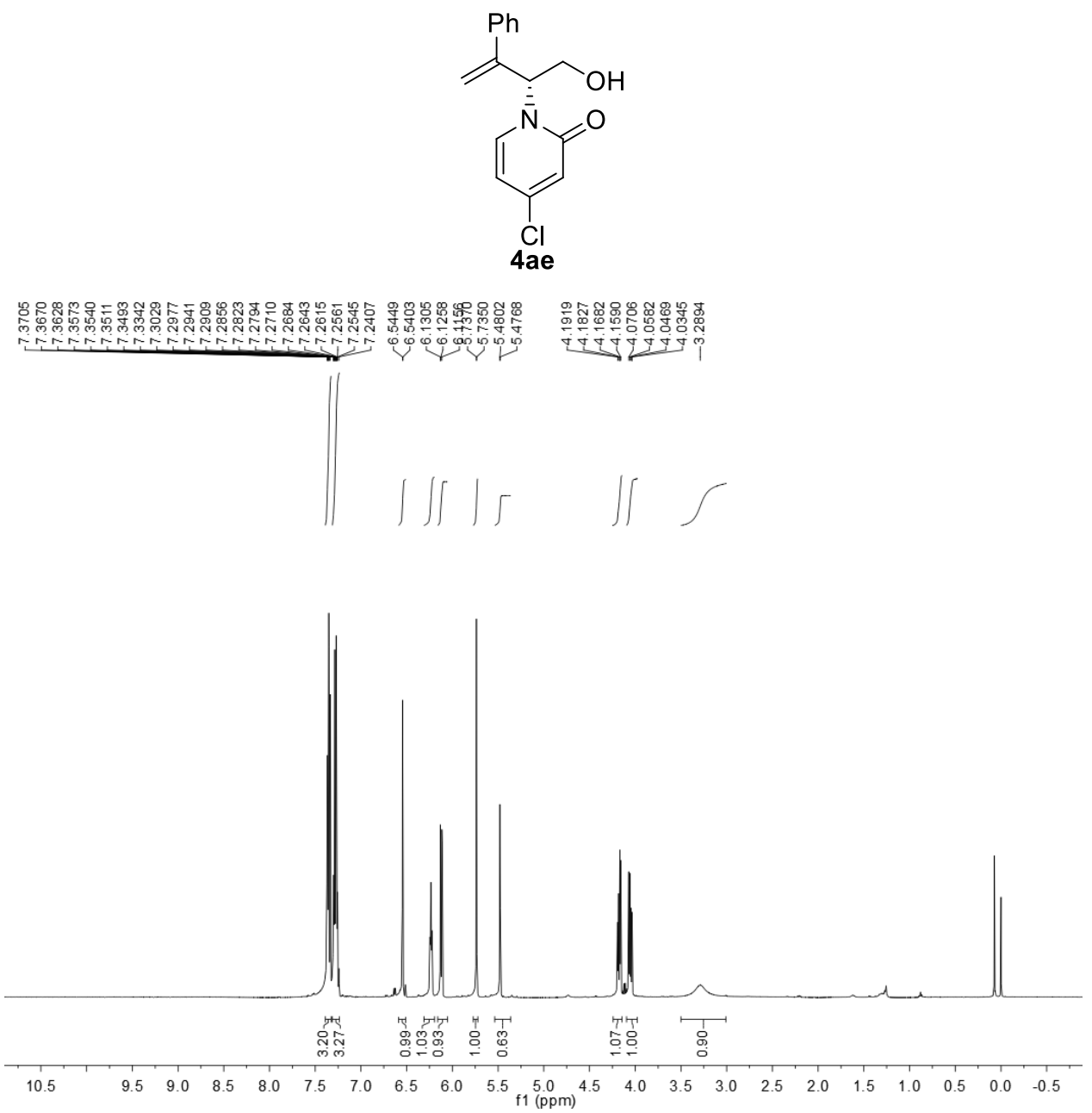

${ }^{1} \mathrm{H}$ NMR spectrum $\left(500 \mathrm{MHz}, \mathrm{CDCl}_{3}\right)$ of compound $4 \mathbf{a e}$
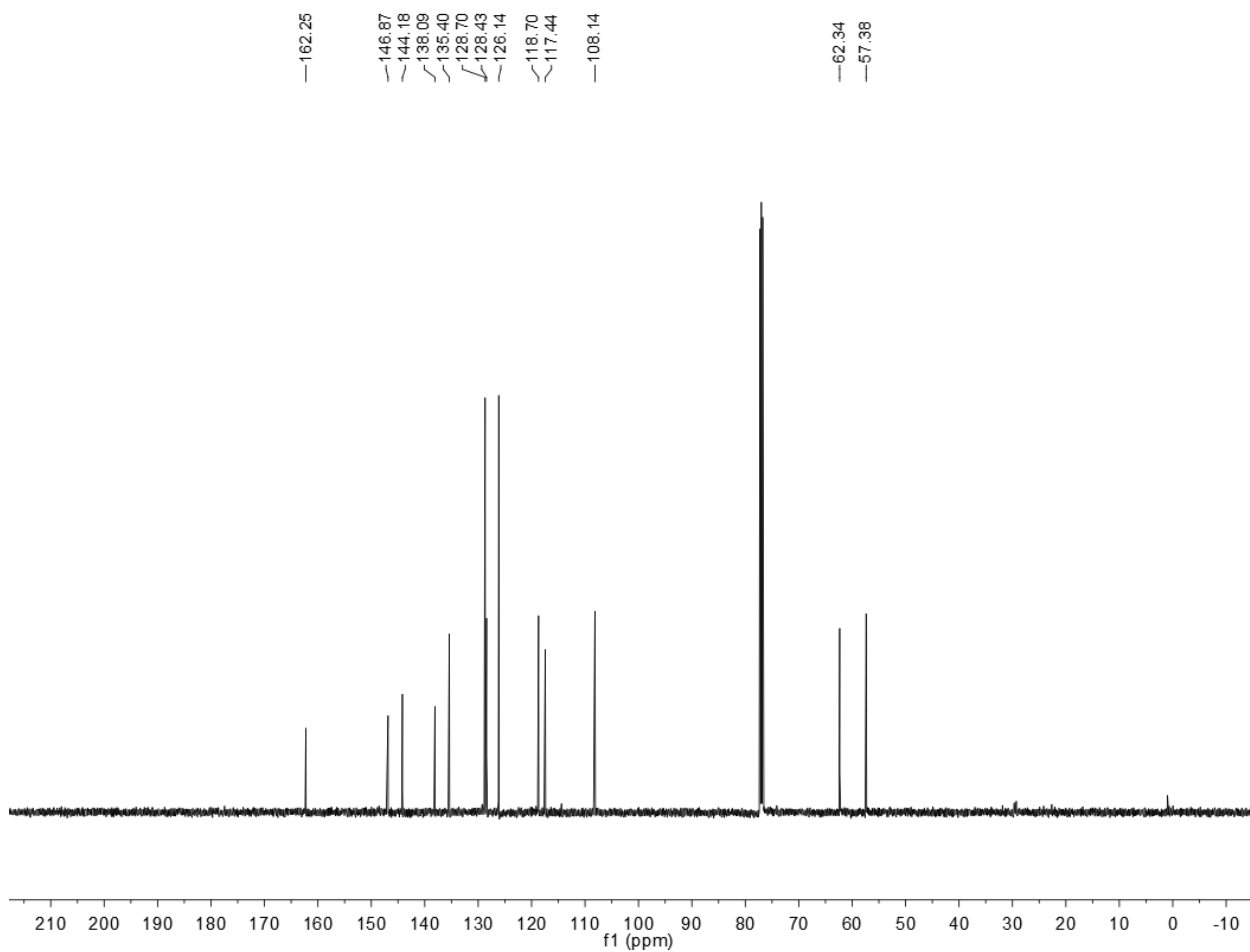

${ }^{13} \mathrm{C}$ NMR spectrum $\left(125 \mathrm{MHz}, \mathrm{CDCl}_{3}\right)$ of compound 4ae 
<smiles>C=C(c1ccccc1)C(CO)n1cc([18O])ccc1=O</smiles>
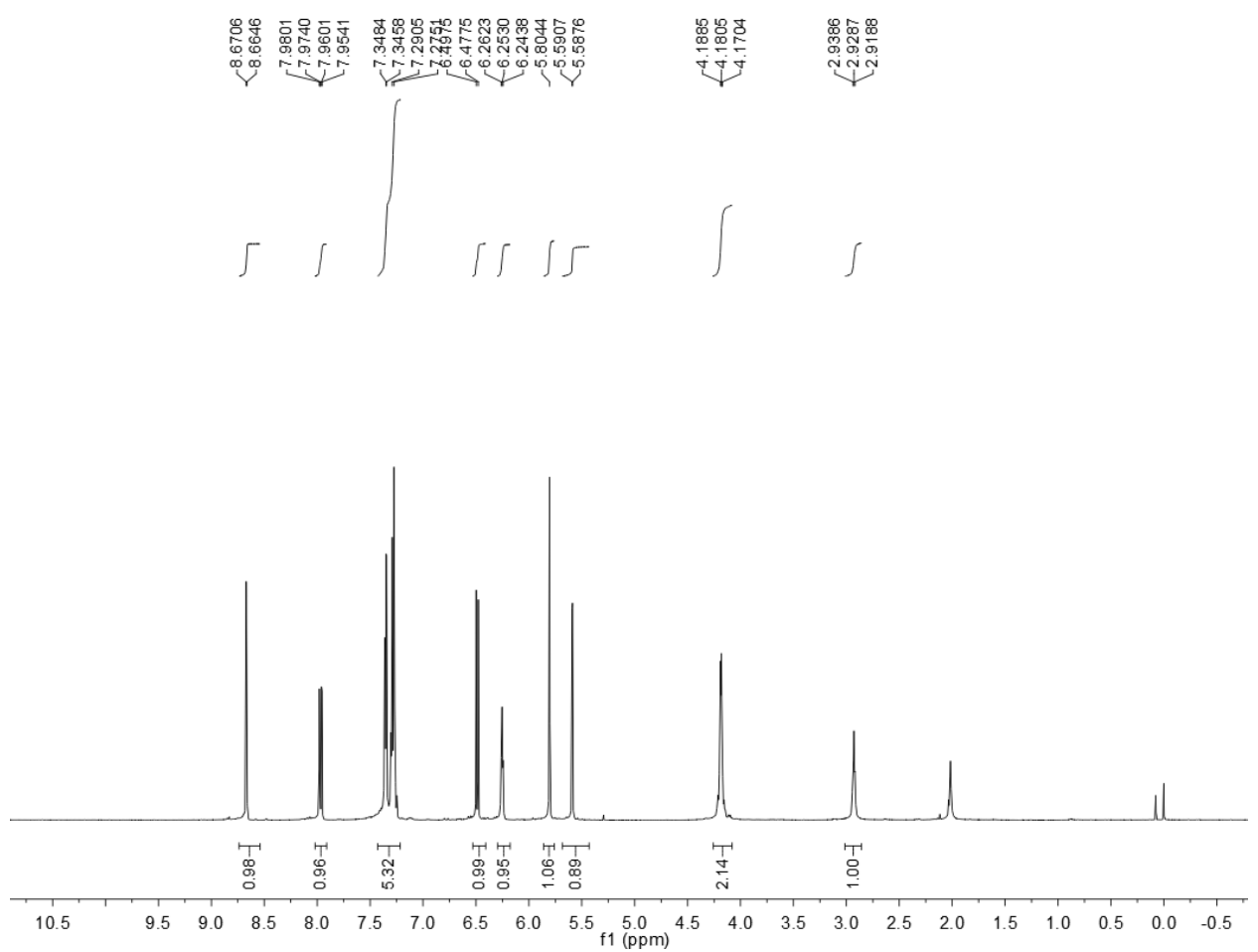

${ }^{1} \mathrm{H}$ NMR spectrum $\left(500 \mathrm{MHz}, \mathrm{CDCl}_{3}\right)$ of compound $\mathbf{4 a f}$

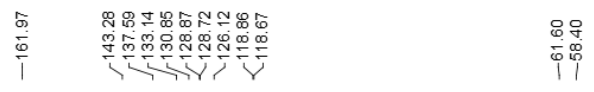

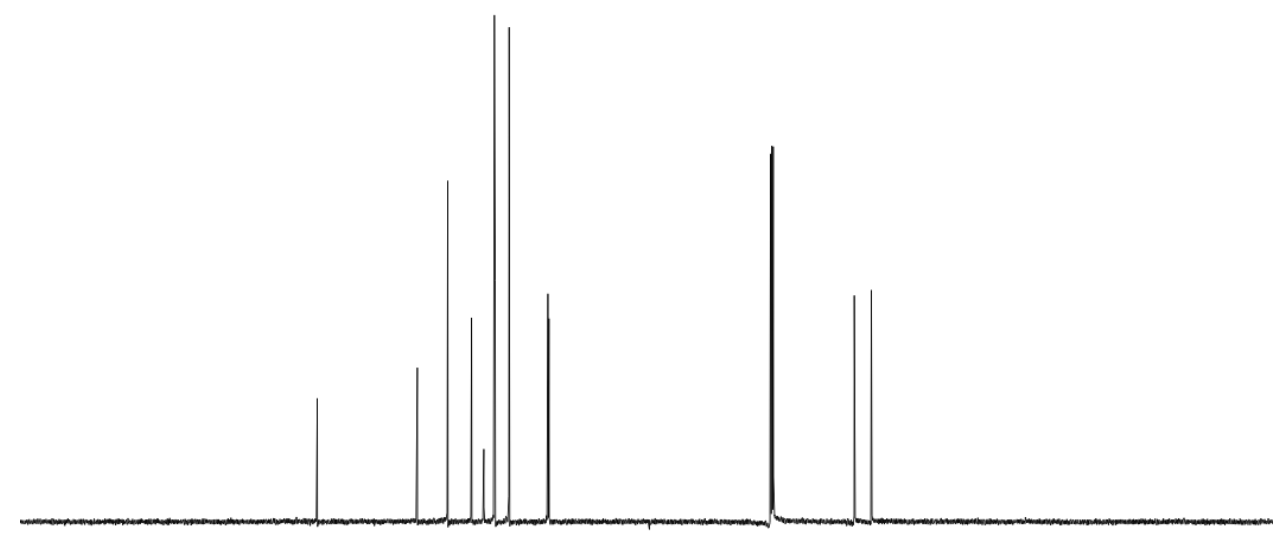

$\begin{array}{llllllllllllllllllllllllll}210 & 200 & 190 & 180 & 170 & 160 & 150 & 140 & 130 & 120 & 110 & 100 & 90 & 80 & 70 & 60 & 50 & 40 & 30 & 20 & 10 & 0 & -10\end{array}$

${ }^{13} \mathrm{C} \mathrm{NMR}$ spectrum $\left(125 \mathrm{MHz}, \mathrm{CDCl}_{3}\right)$ of compound 4af 

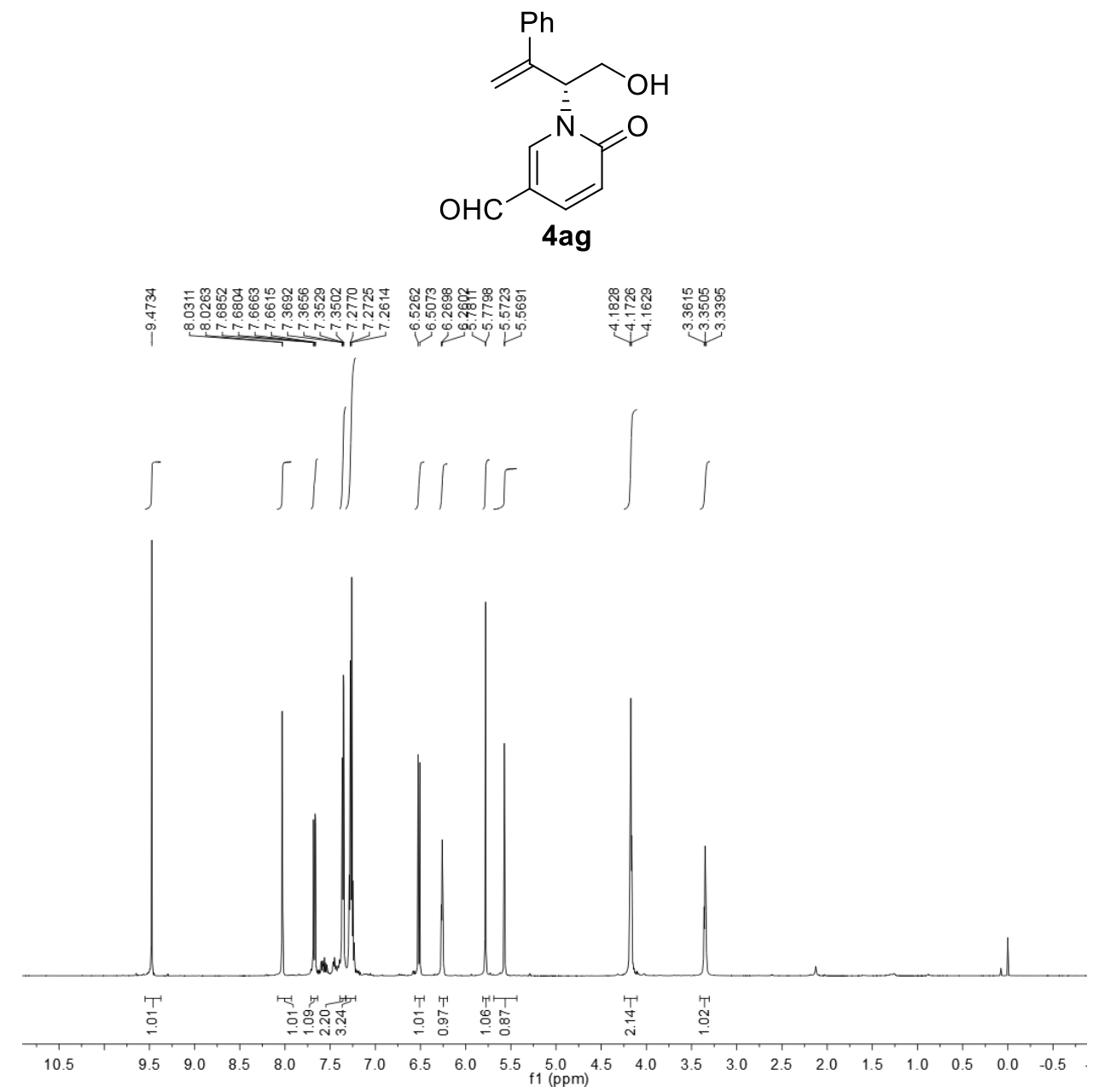

${ }^{1} \mathrm{H}$ NMR spectrum $\left(500 \mathrm{MHz}, \mathrm{CDCl}_{3}\right)$ of compound $\mathbf{4 a g}$

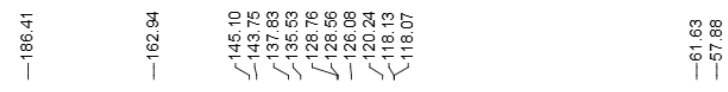

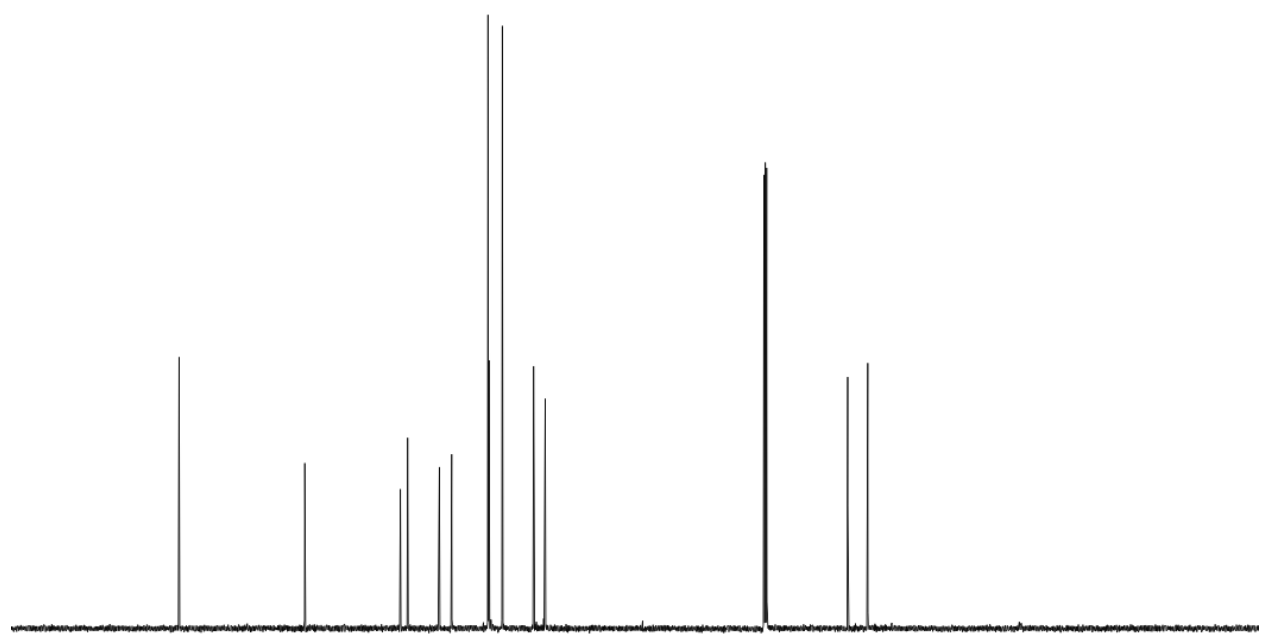

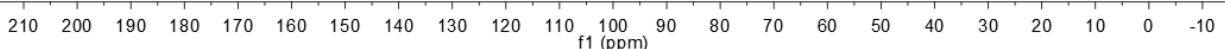

${ }^{13} \mathrm{C}$ NMR spectrum $\left(125 \mathrm{MHz}, \mathrm{CDCl}_{3}\right)$ of compound $4 \mathbf{a g}$ 

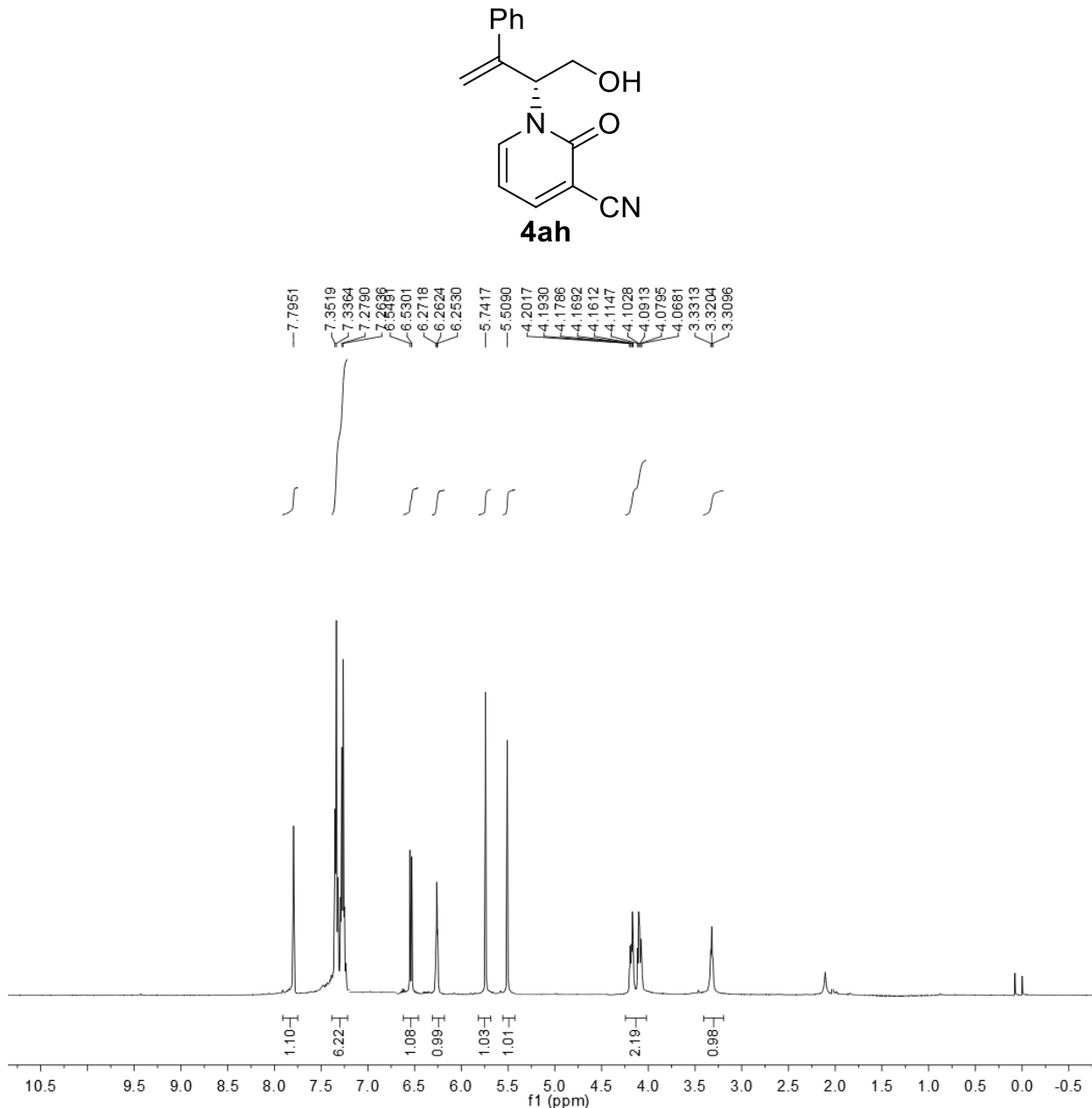

${ }^{1} \mathrm{H}$ NMR spectrum $\left(500 \mathrm{MHz}, \mathrm{CDCl}_{3}\right)$ of compound $\mathbf{4 a h}$

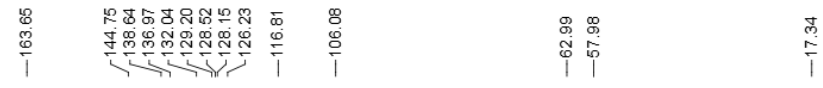

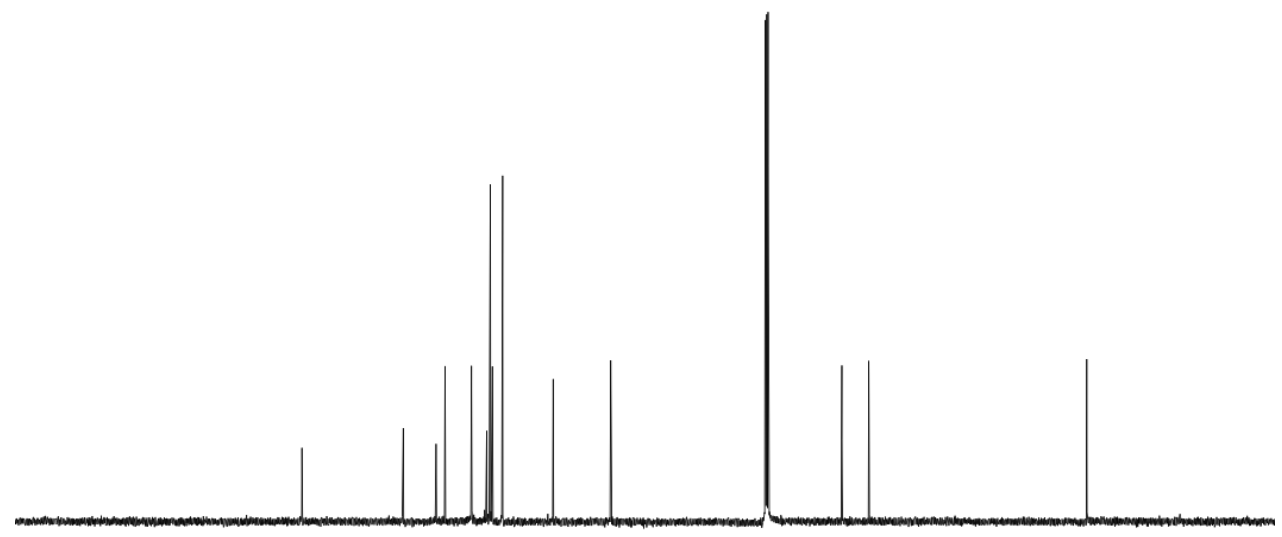

$\begin{array}{lllllllllllllllllllllll}210 & 200 & 190 & 180 & 170 & 160 & 150 & 140 & 130 & 120 & 110 & 100 & 90 & 80 & 70 & 60 & 50 & 40 & 30 & 20 & 10 & 0 & -10\end{array}$

${ }^{13} \mathrm{C}$ NMR spectrum (125 MHz, $\mathrm{CDCl}_{3}$ ) of compound 4ah 
<smiles>C=C(c1ccccc1)C(CO)n1cc(Br)c(Br)c(Br)c1=O</smiles>

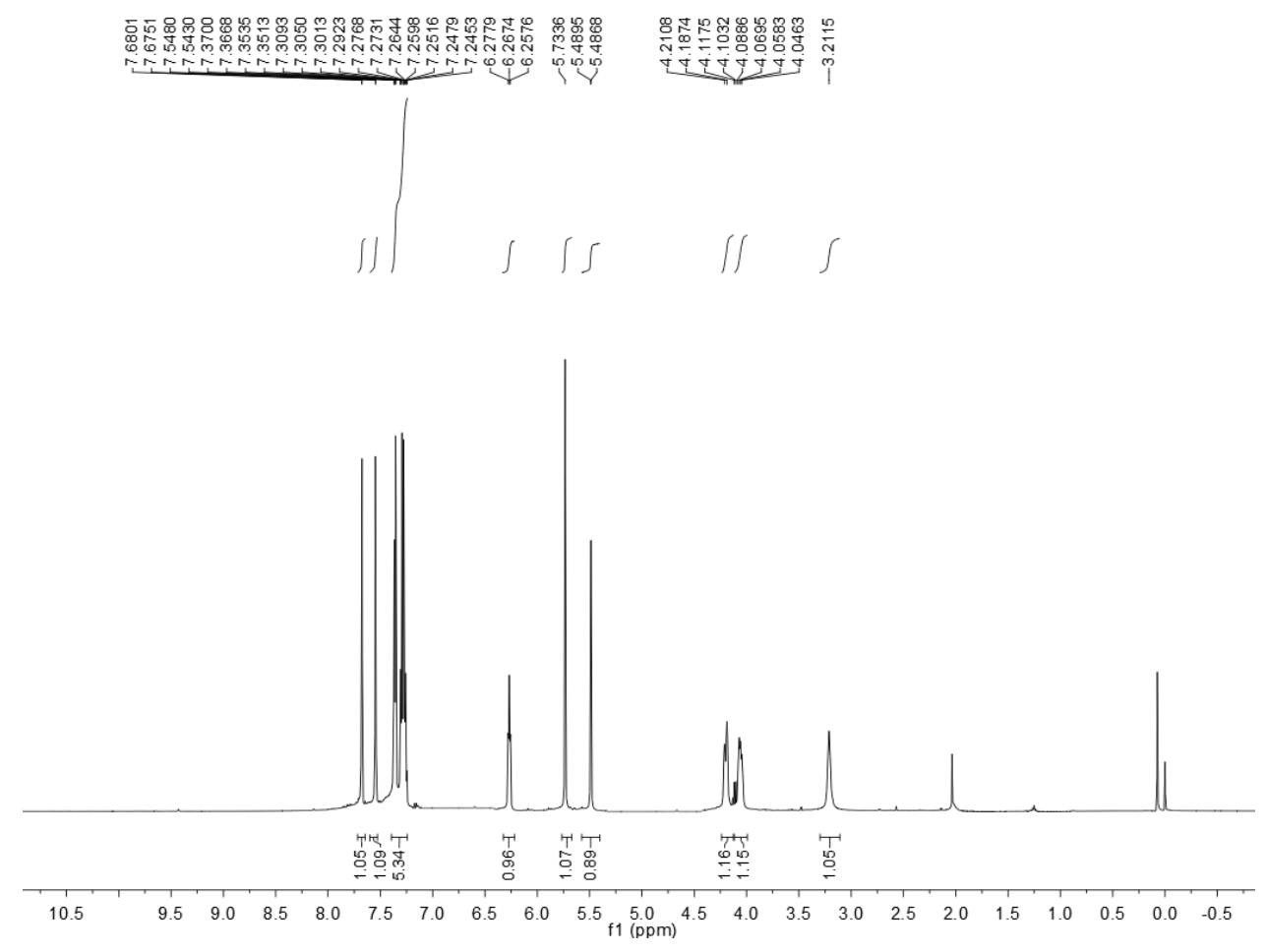

${ }^{1} \mathrm{H}$ NMR spectrum $\left(500 \mathrm{MHz}, \mathrm{CDCl}_{3}\right)$ of compound 4ai

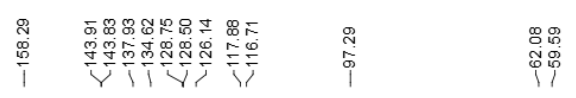

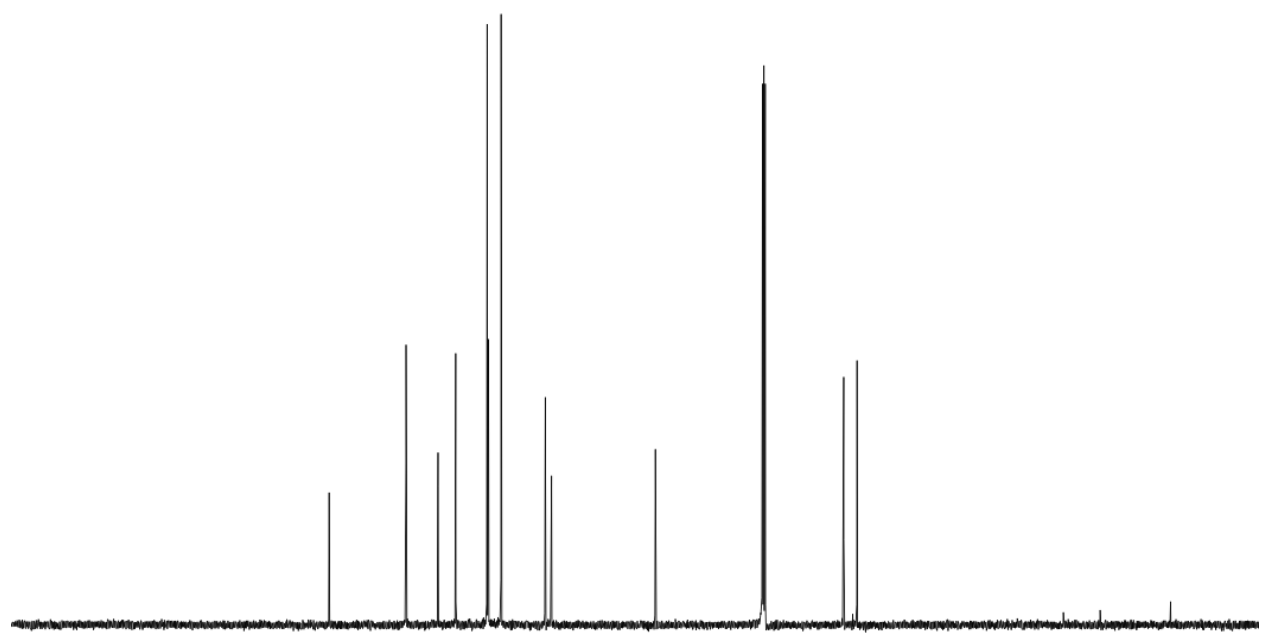

$\begin{array}{llllllllllllllllllllllllll}210 & 200 & 190 & 180 & 170 & 160 & 150 & 140 & 130 & 120 & 110 & 100 & 90 & 80 & 70 & 60 & 50 & 40 & 30 & 20 & 10 & 0 & -10\end{array}$

${ }^{13} \mathrm{C}$ NMR spectrum $\left(125 \mathrm{MHz}, \mathrm{CDCl}_{3}\right)$ of compound 4ai 


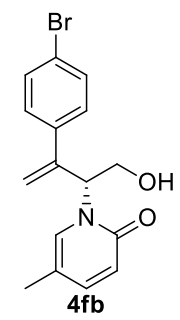

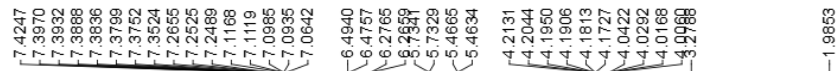
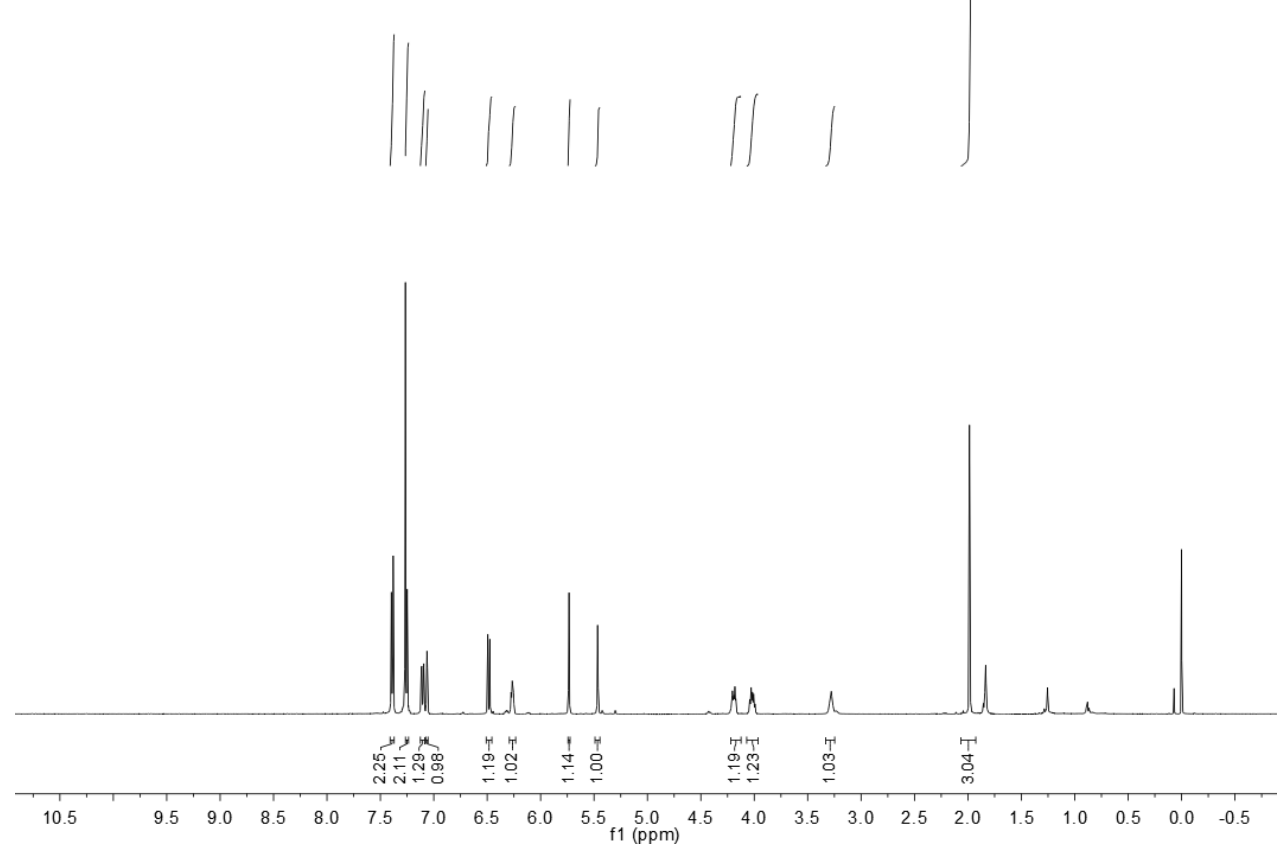

${ }^{1} \mathrm{H}$ NMR spectrum $\left(500 \mathrm{MHz}, \mathrm{CDCl}_{3}\right)$ of compound $\mathbf{4 f b}$

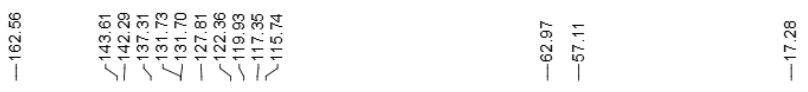

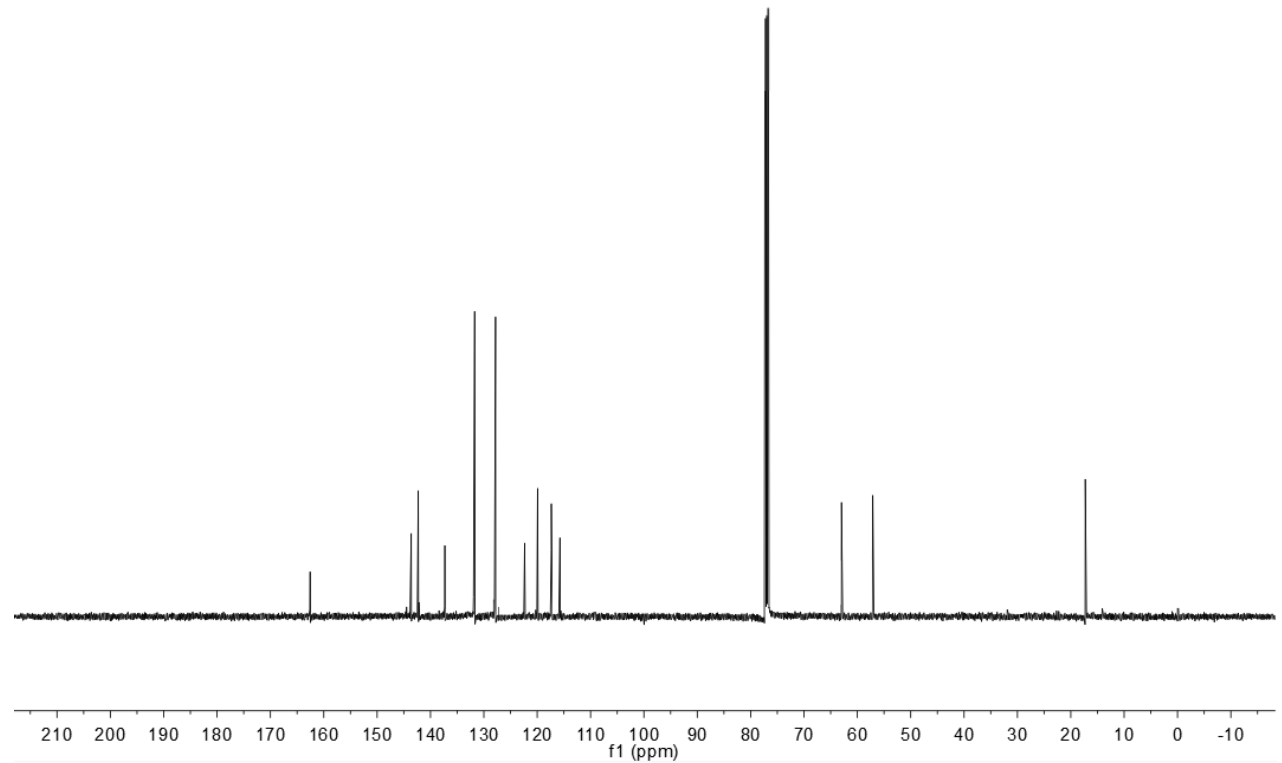

${ }^{13} \mathrm{C} \mathrm{NMR}$ spectrum $\left(125 \mathrm{MHz}, \mathrm{CDCl}_{3}\right)$ of compound $\mathbf{4 f b}$ 

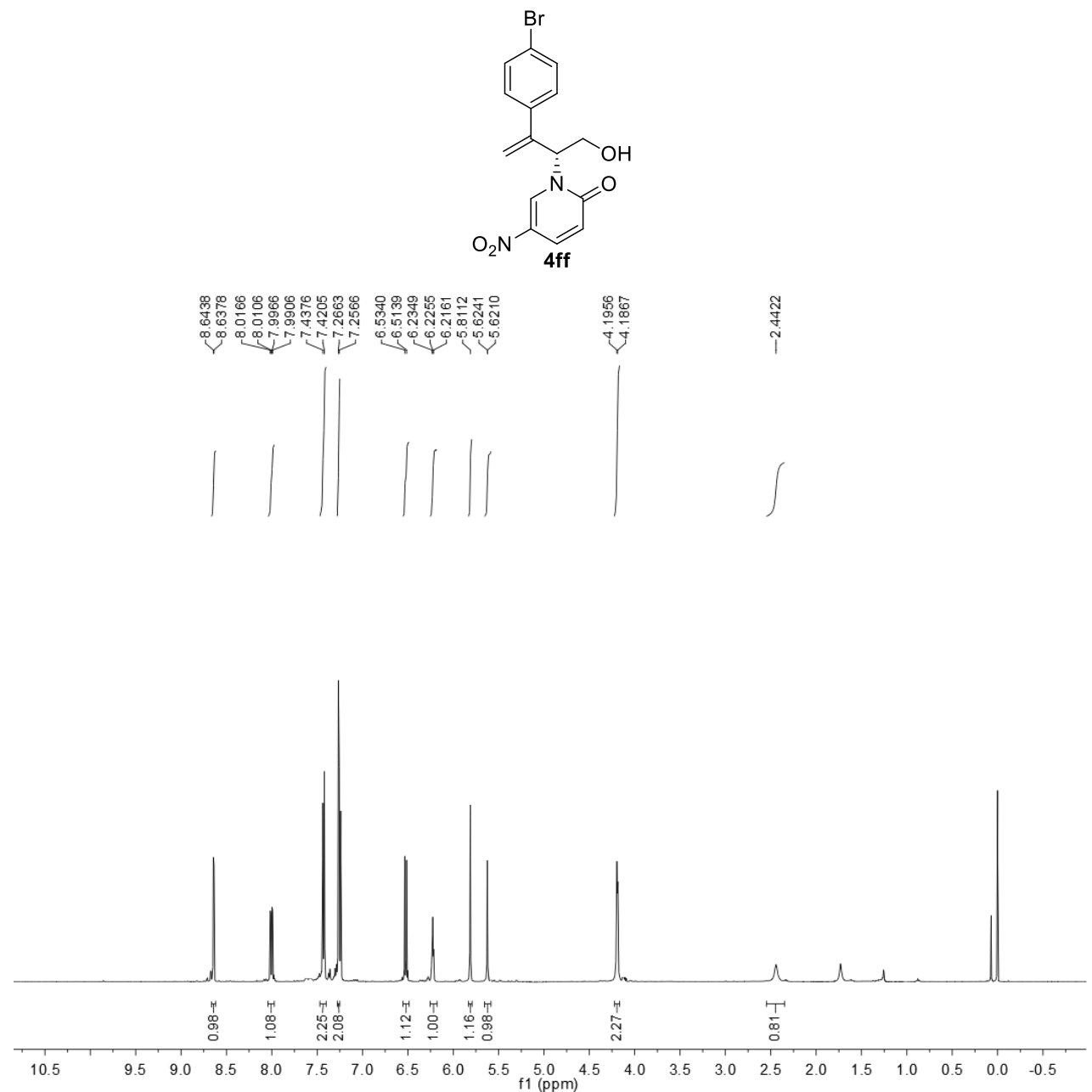

${ }^{1} \mathrm{H}$ NMR spectrum $\left(500 \mathrm{MHz}, \mathrm{CDCl}_{3}\right.$ ) of compound $\mathbf{4 f f}$

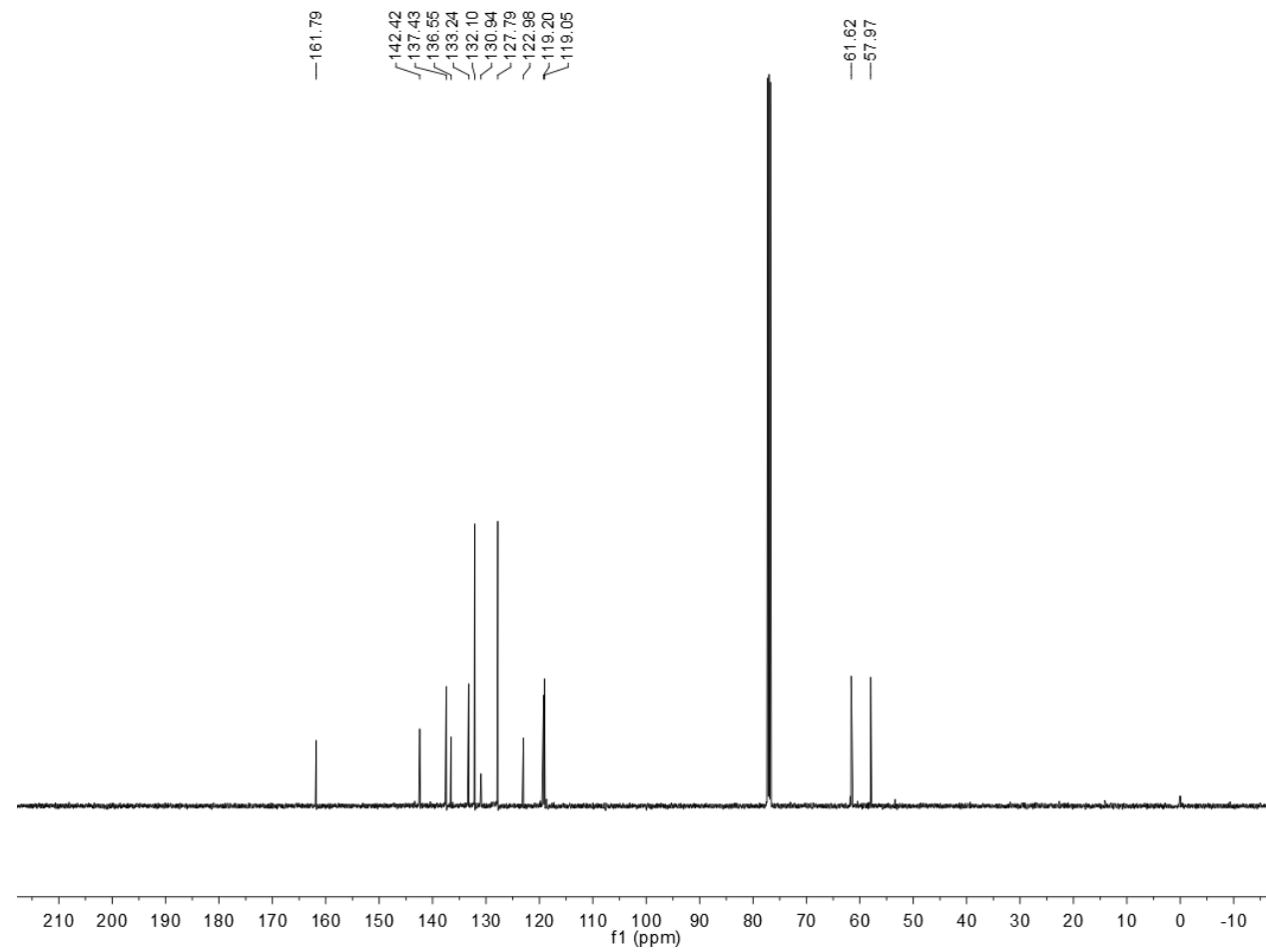

${ }^{13} \mathrm{C}$ NMR spectrum $\left(125 \mathrm{MHz}, \mathrm{CDCl}_{3}\right)$ of compound $\mathbf{4 f f}$ 


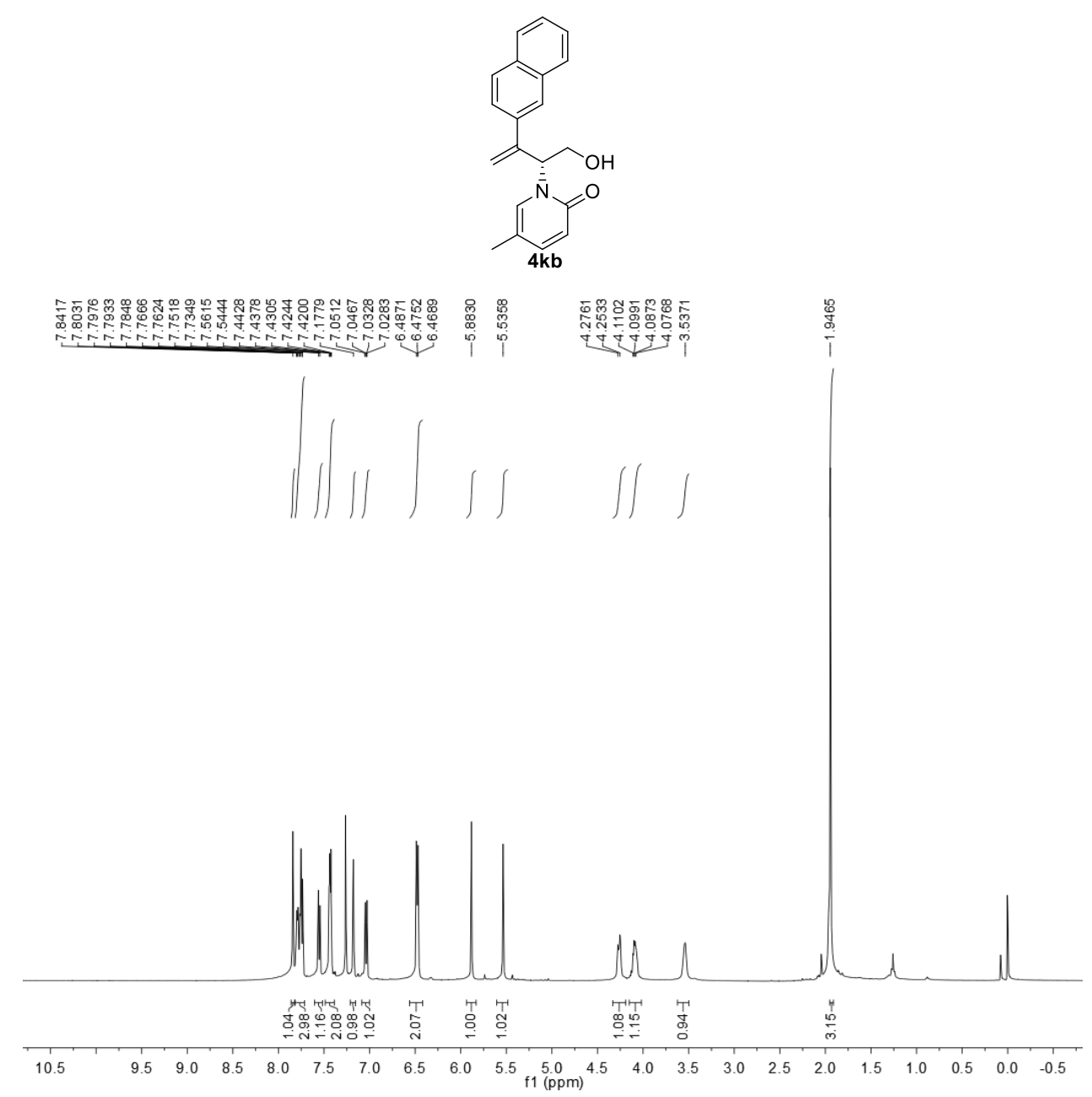

${ }^{1} \mathrm{H}$ NMR spectrum $\left(500 \mathrm{MHz}, \mathrm{CDCl}_{3}\right.$ ) of compound $\mathbf{4 k \mathbf { k b }}$

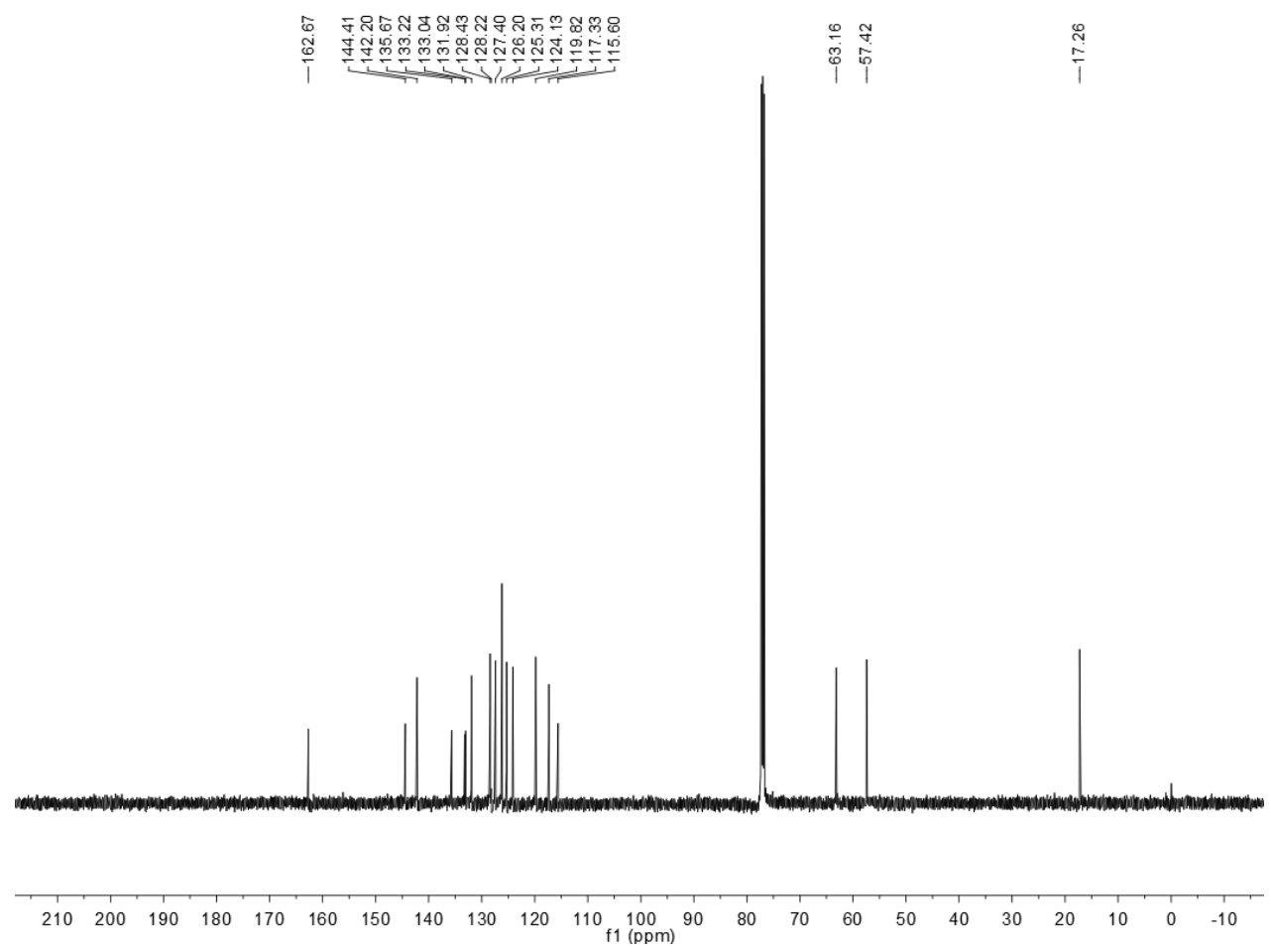

${ }^{13} \mathrm{C}$ NMR spectrum $\left(125 \mathrm{MHz}, \mathrm{CDCl}_{3}\right)$ of compound $\mathbf{4 k b}$ 

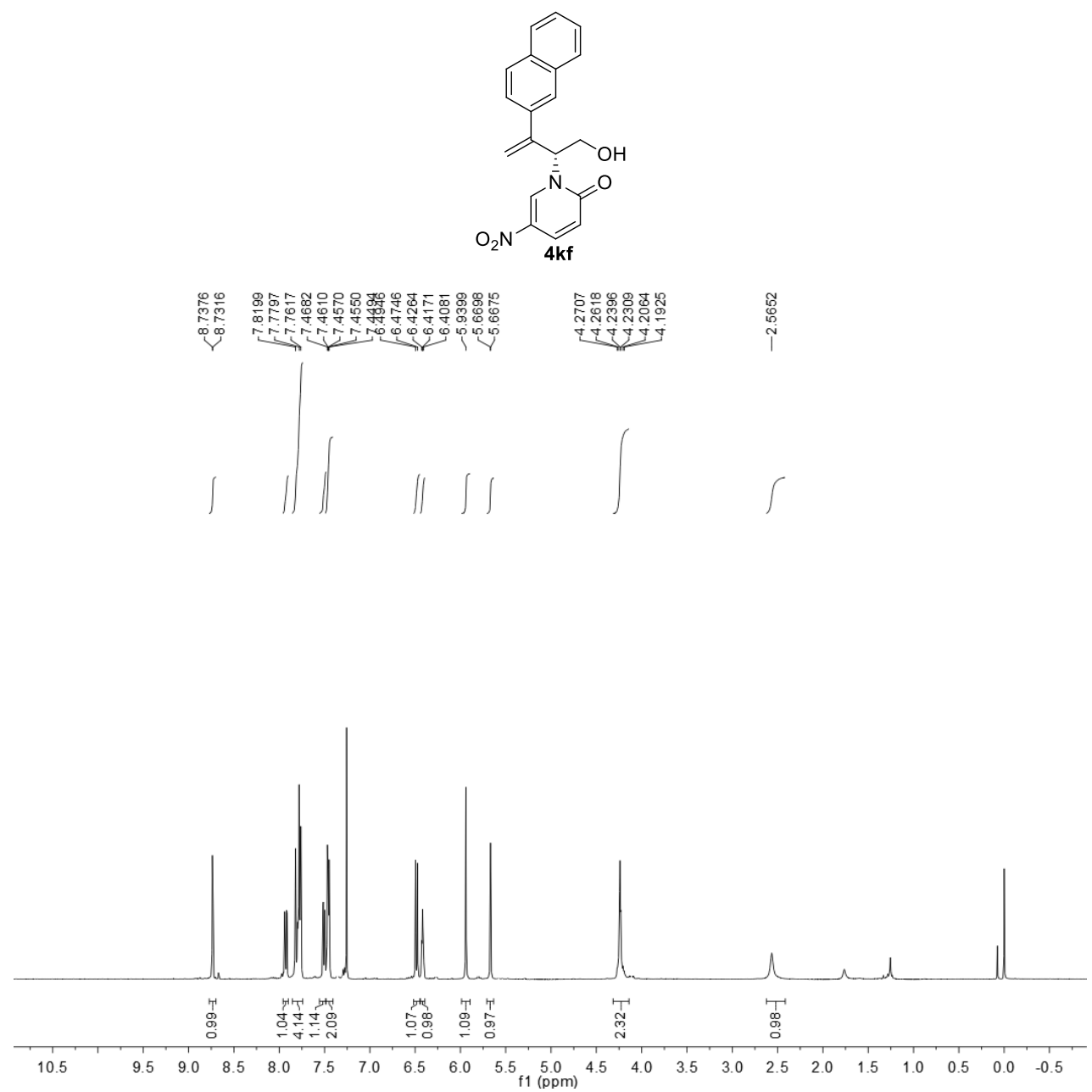

${ }^{1} \mathrm{H}$ NMR spectrum $\left(500 \mathrm{MHz}, \mathrm{CDCl}_{3}\right)$ of compound $\mathbf{4 k f}$

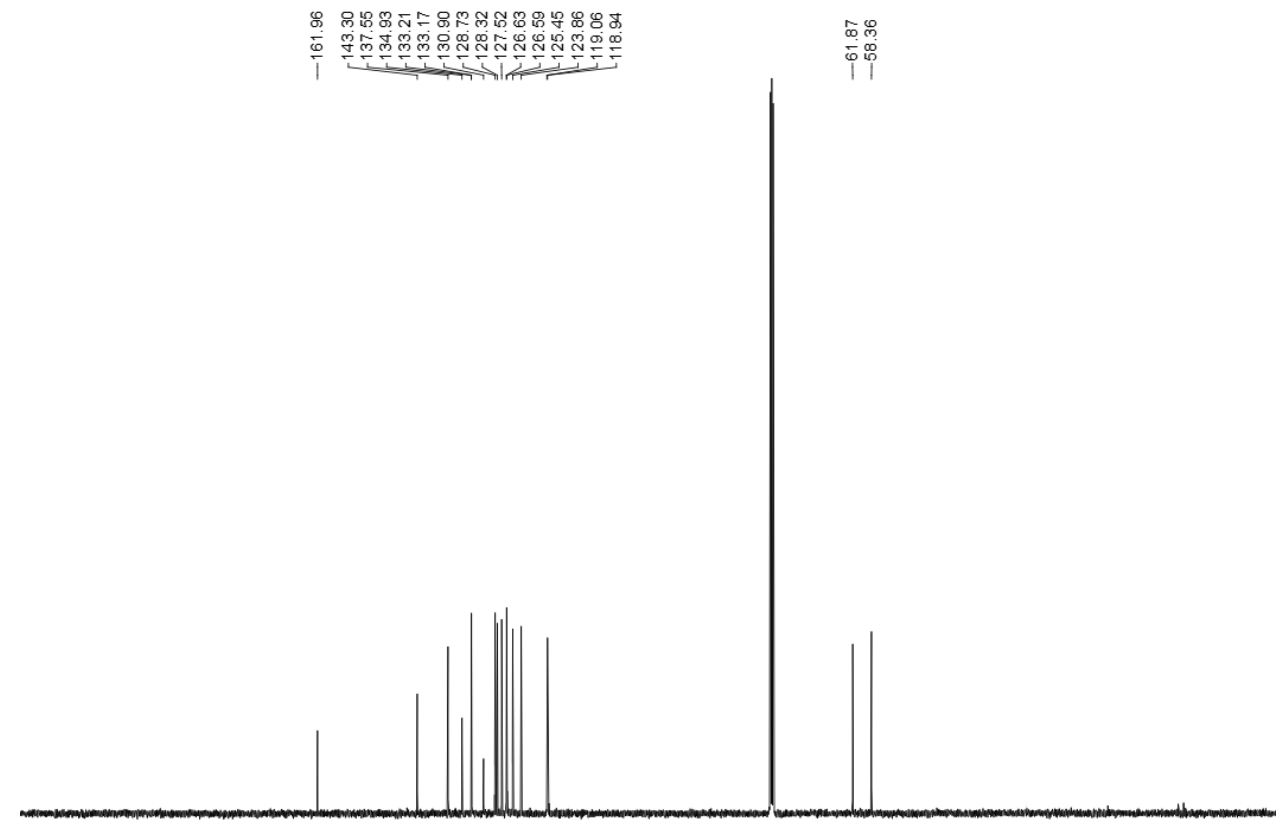

$\begin{array}{llllllllllllllllllllllllllll}210 & 200 & 190 & 180 & 170 & 160 & 150 & 140 & 130 & 120 & 110 & 100 & 90 & 80 & 70 & 60 & 50 & 40 & 30 & 20 & 10 & 0 & -10\end{array}$

${ }^{13} \mathrm{C}$ NMR spectrum $\left(125 \mathrm{MHz}, \mathrm{CDCl}_{3}\right)$ of compound $\mathbf{4 k f}$ 

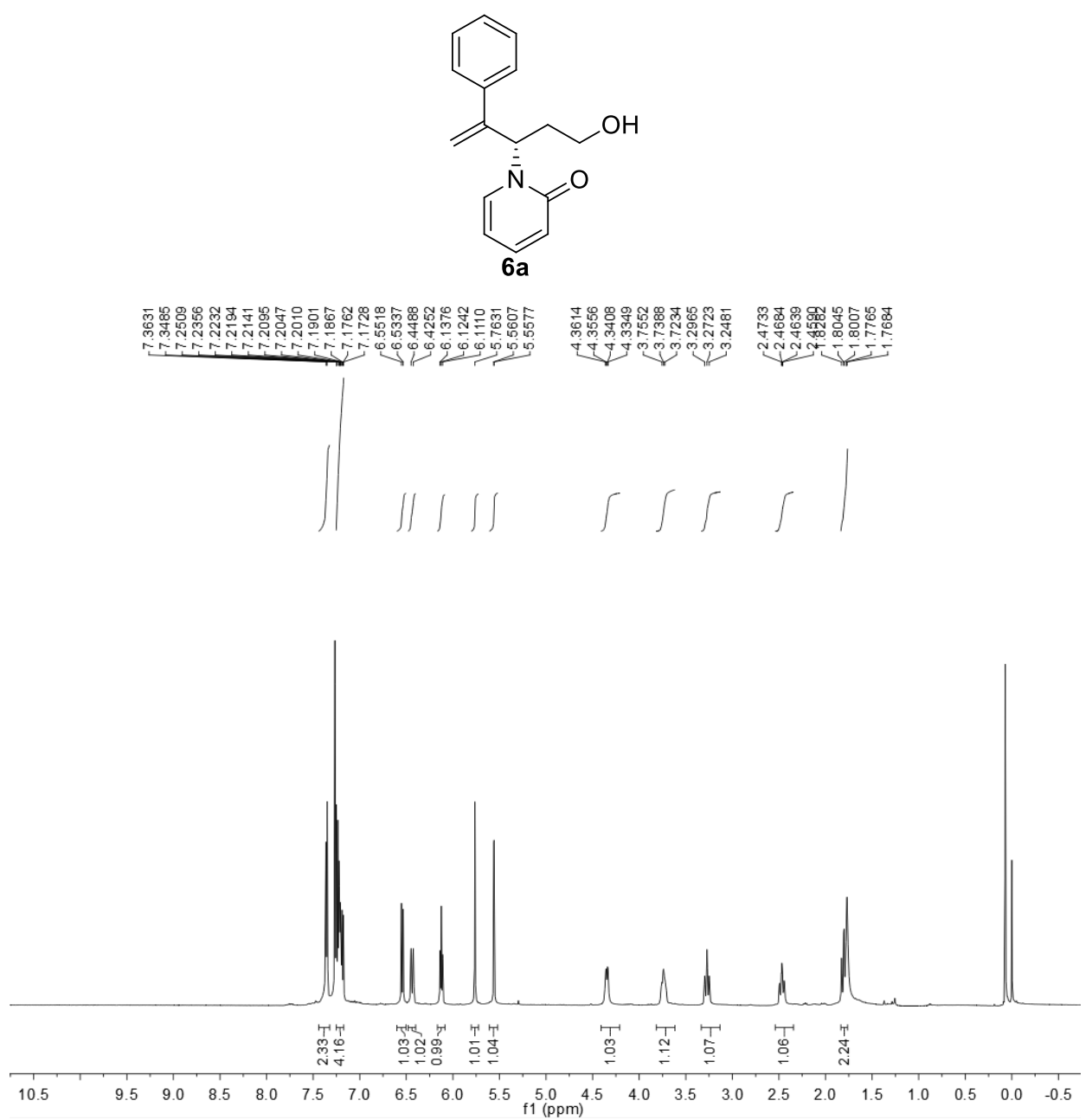

${ }^{1} \mathrm{H}$ NMR spectrum $\left(500 \mathrm{MHz}, \mathrm{CDCl}_{3}\right)$ of compound $\mathbf{6 a}$

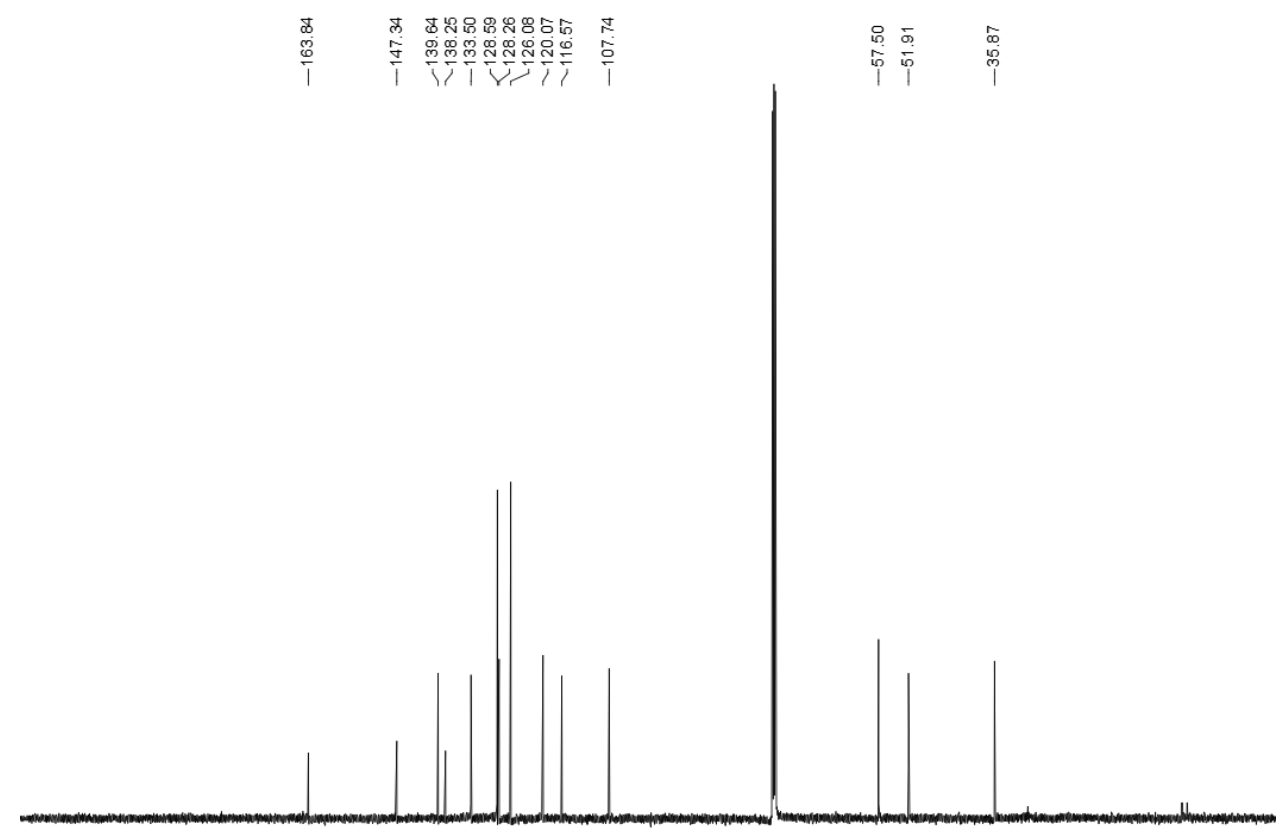

$\begin{array}{llllllllllllllllllllllllllll}210 & 200 & 190 & 180 & 170 & 160 & 150 & 140 & 130 & 120 & 110 & 100 & 90 & 80 & 70 & 60 & 50 & 40 & 30 & 20 & 10 & 0 & -10\end{array}$

${ }^{13} \mathrm{C}$ NMR spectrum $\left(125 \mathrm{MHz}, \mathrm{CDCl}_{3}\right)$ of compound $\mathbf{6 a}$ 
<smiles>C=C(c1ccccc1)[C@@H](CCCO)N1C=C(C)[C+]=CC1=O</smiles>

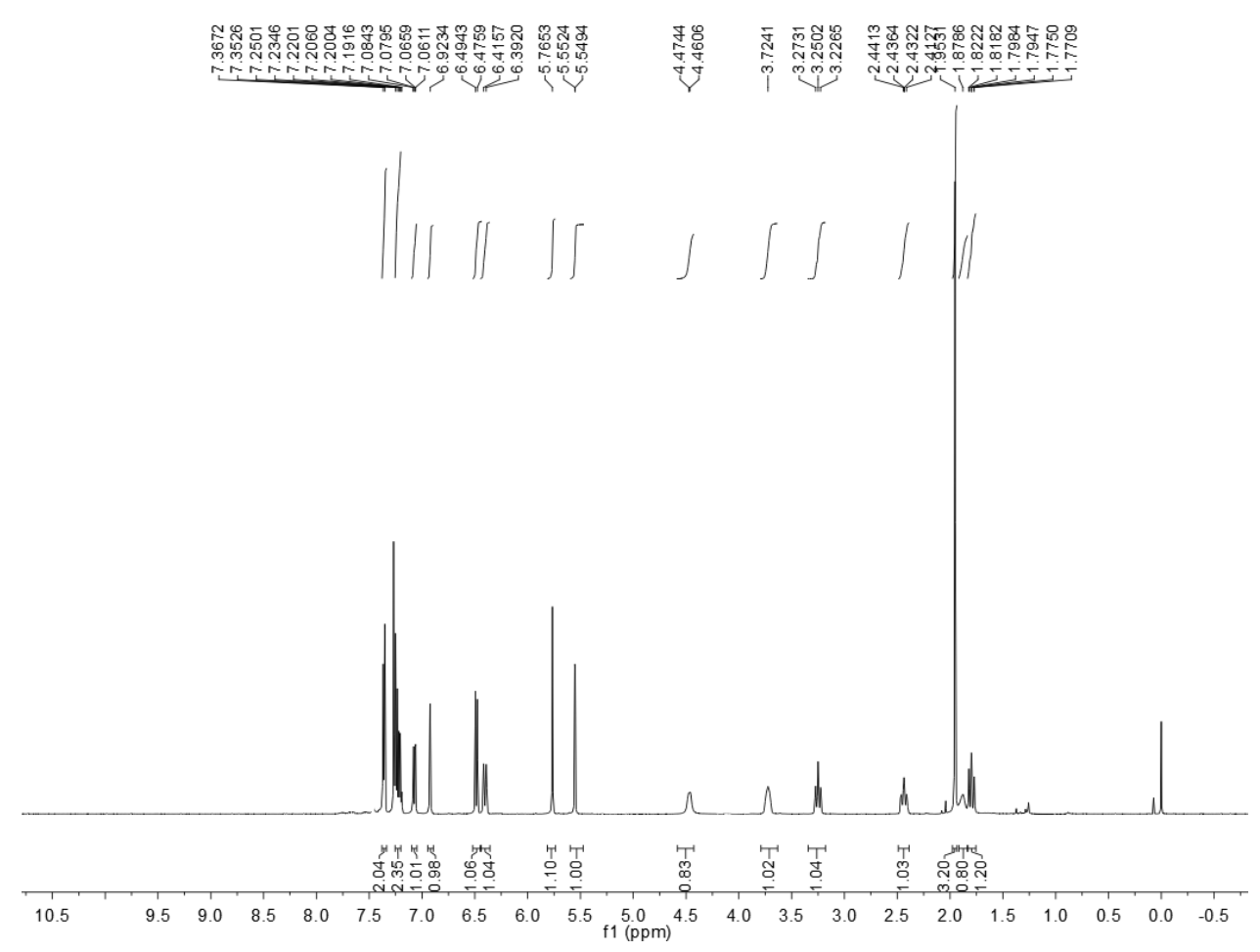

${ }^{1} \mathrm{H}$ NMR spectrum $\left(500 \mathrm{MHz}, \mathrm{CDCl}_{3}\right.$ ) of compound $\mathbf{6 b}$

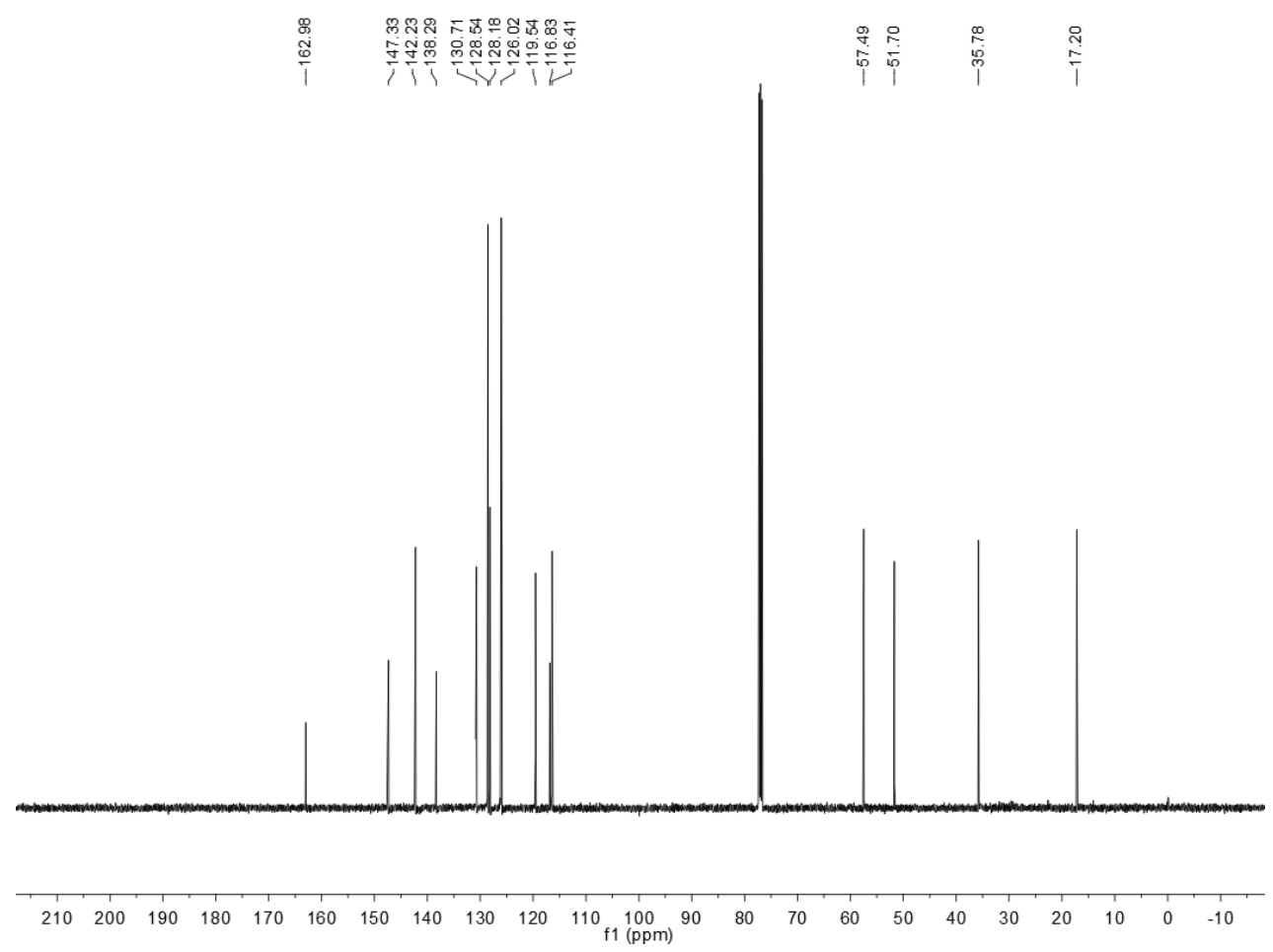

${ }^{13} \mathrm{C}$ NMR spectrum $\left(125 \mathrm{MHz}, \mathrm{CDCl}_{3}\right.$ ) of compound $\mathbf{6 b}$ 
<smiles>C=C(c1ccccc1)C(CCO)N1C=C(C=O)CCC1=O</smiles>
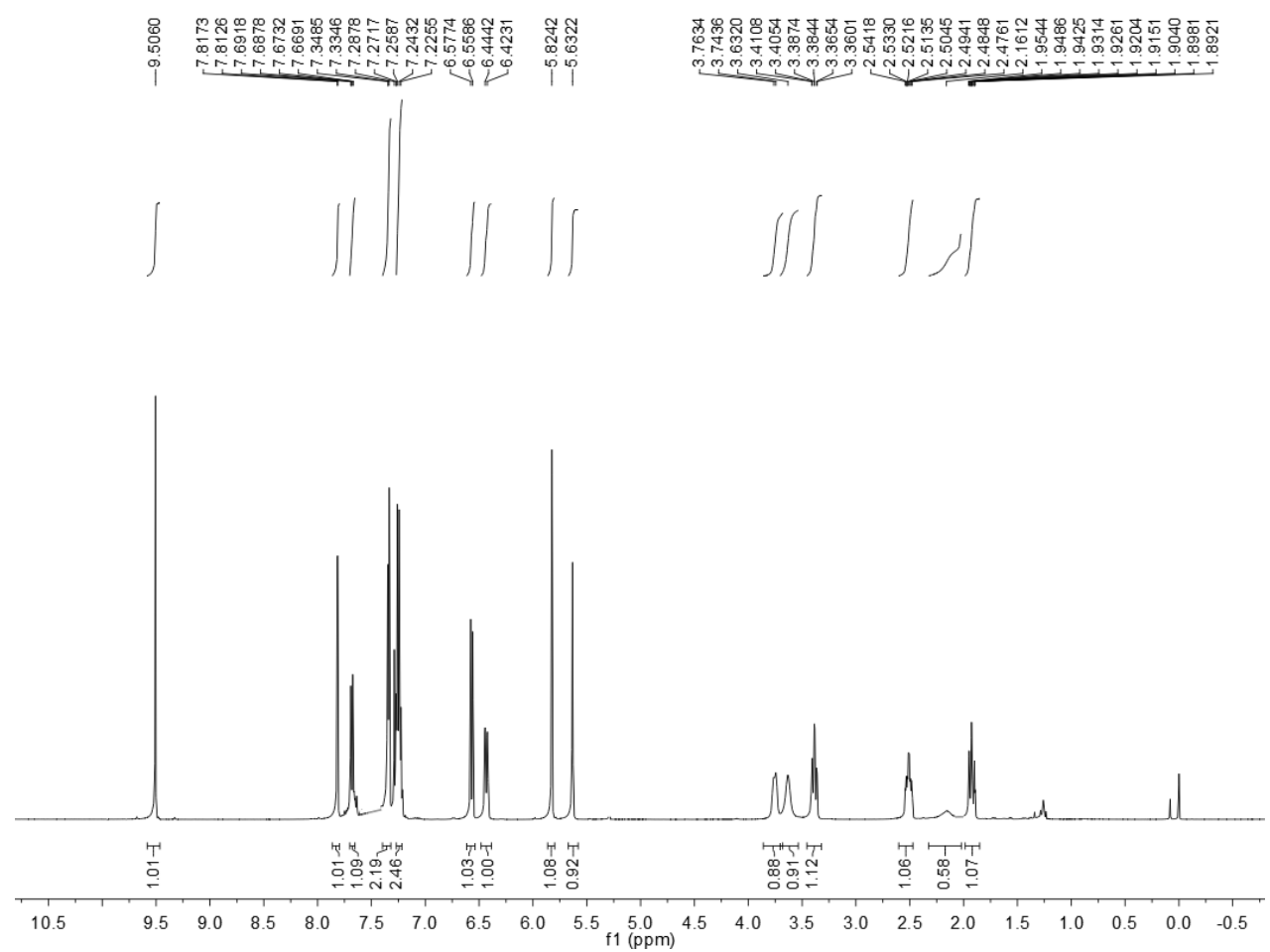

${ }^{1} \mathrm{H}$ NMR spectrum $\left(500 \mathrm{MHz}, \mathrm{CDCl}_{3}\right.$ ) of compound $\mathbf{6 c}$

\begin{tabular}{|c|c|c|}
\hline$\frac{1}{0}$ & 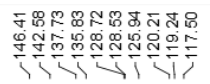 & 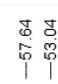 \\
\hline
\end{tabular}

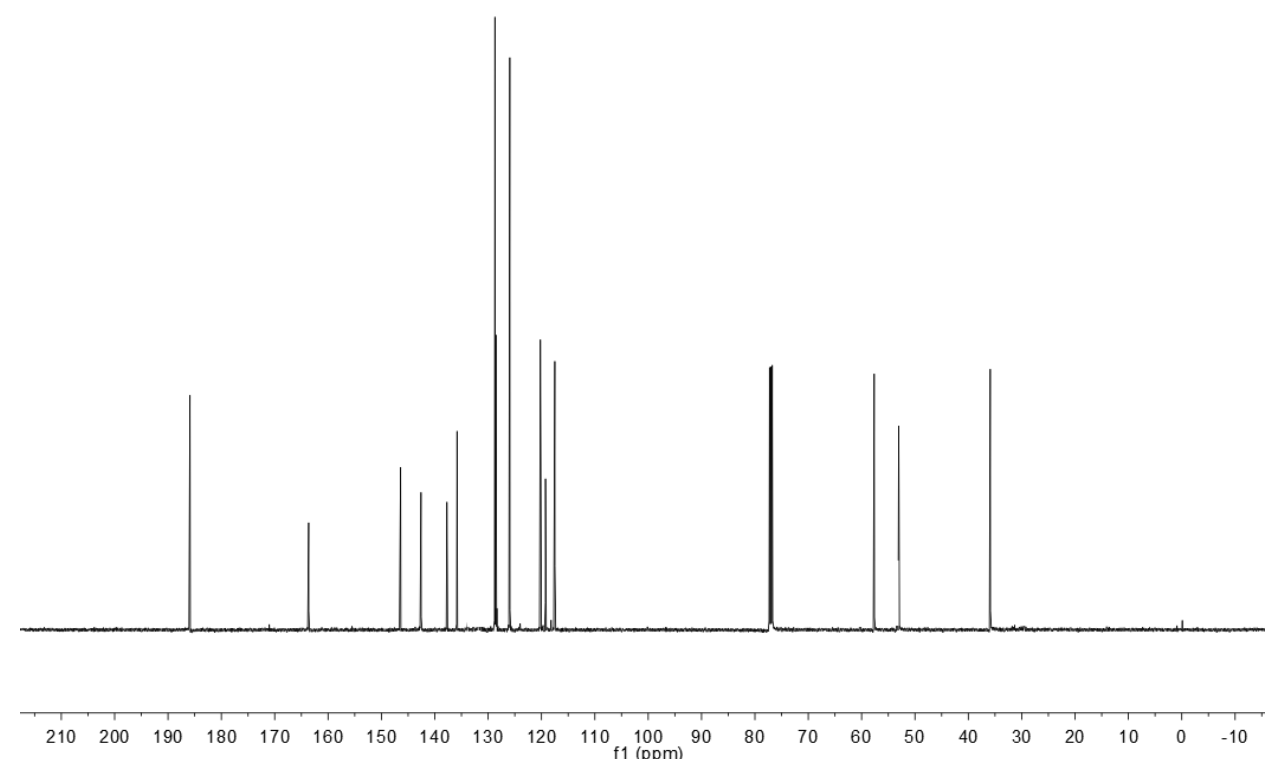

${ }^{13} \mathrm{C}$ NMR spectrum $\left(125 \mathrm{MHz}, \mathrm{CDCl}_{3}\right)$ of compound $\mathbf{6 c}$ 


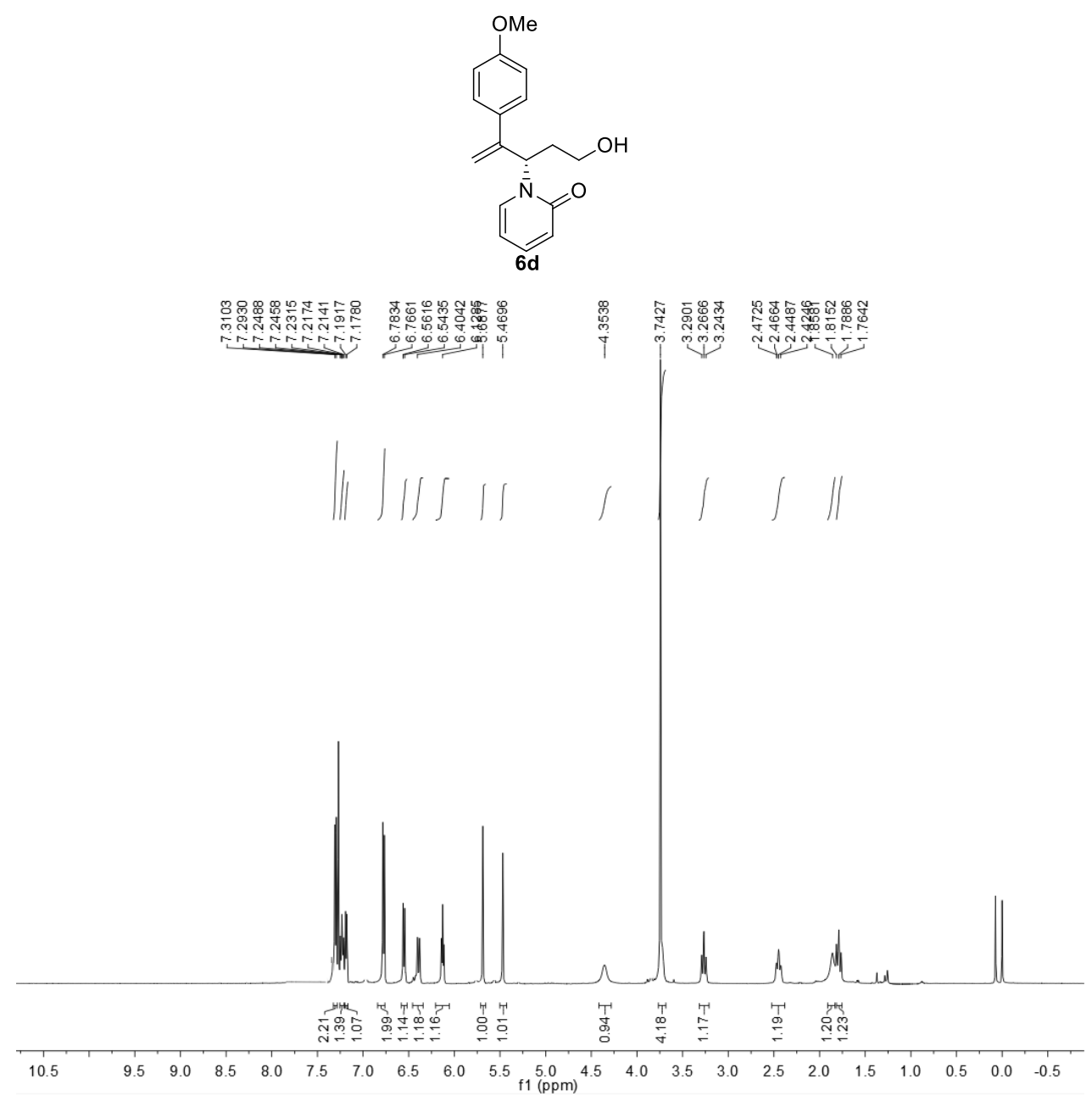

${ }^{1} \mathrm{H}$ NMR spectrum $\left(500 \mathrm{MHz}, \mathrm{CDCl}_{3}\right.$ ) of compound $\mathbf{6 d}$

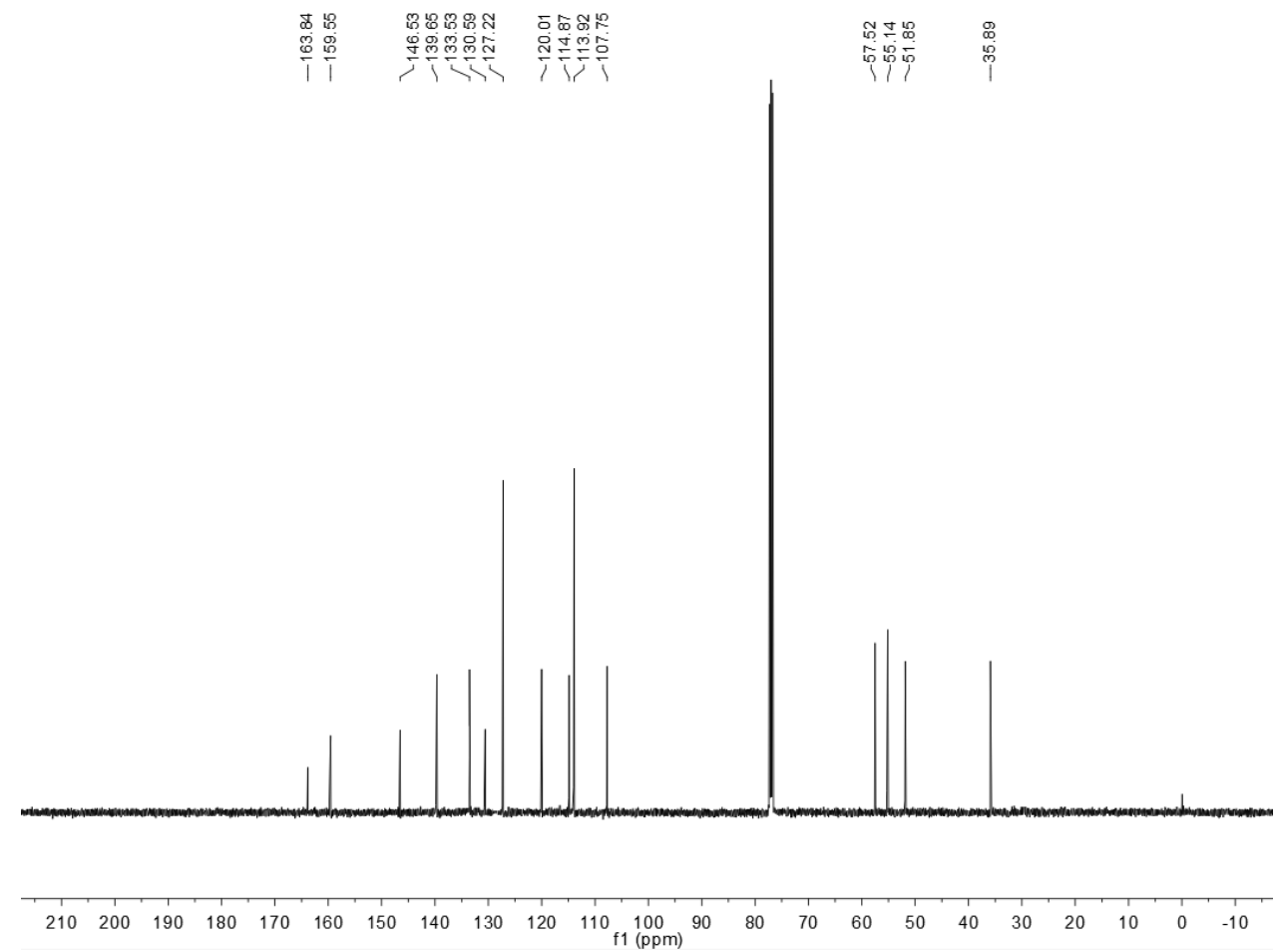

${ }^{13} \mathrm{C}$ NMR spectrum $\left(125 \mathrm{MHz}, \mathrm{CDCl}_{3}\right)$ of compound $\mathbf{6 d}$ 


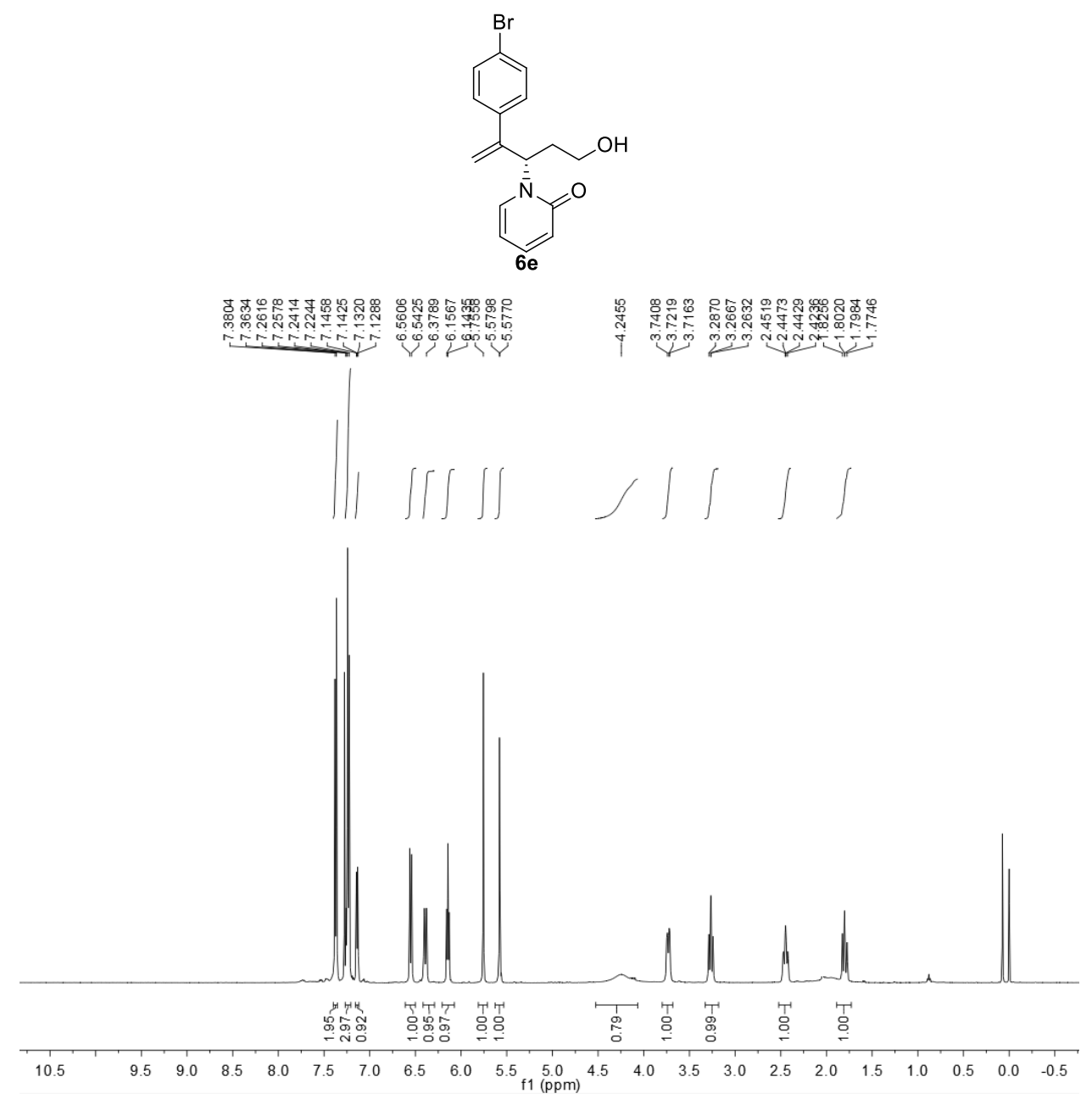

${ }^{1} \mathrm{H} \mathrm{NMR}$ spectrum $\left(500 \mathrm{MHz}, \mathrm{CDCl}_{3}\right)$ of compound $\mathbf{6 e}$

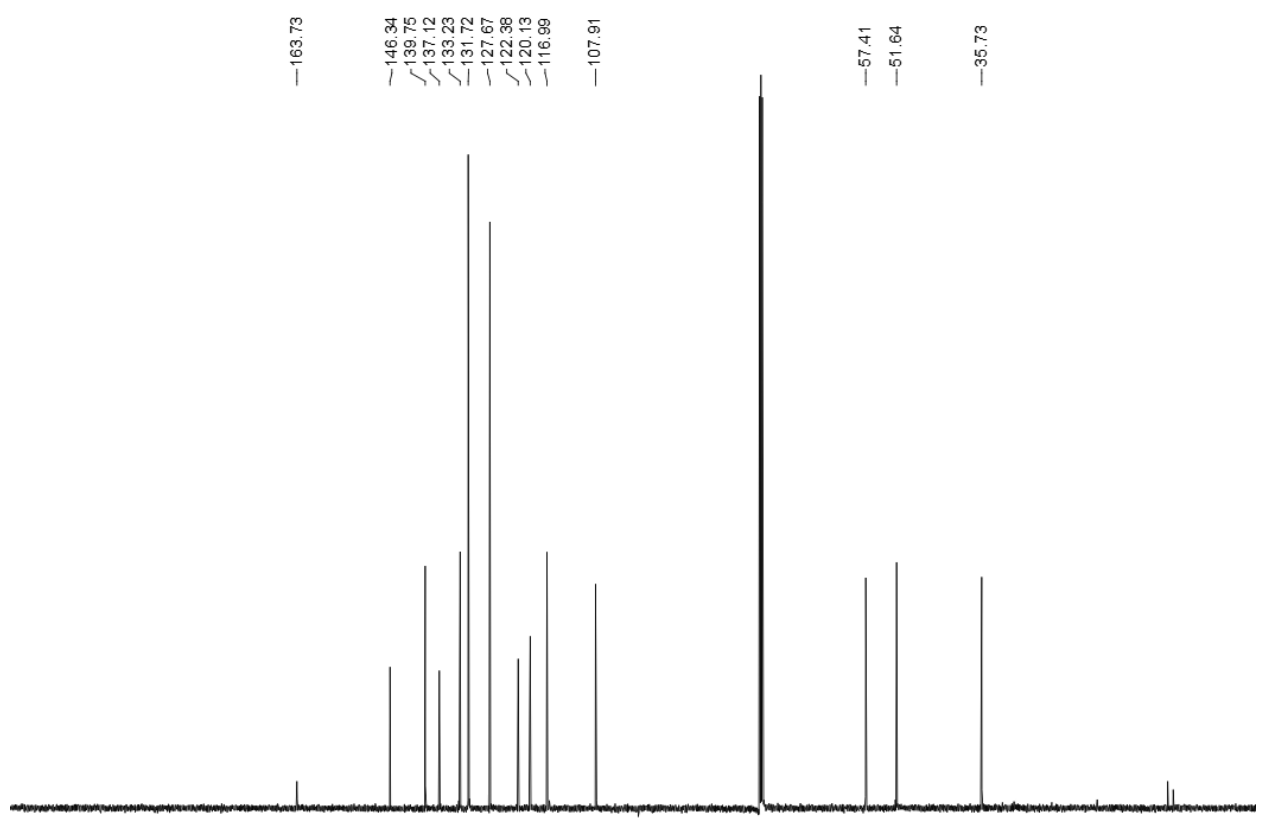

$\begin{array}{llllllllllllllllllllllllllll}210 & 200 & 190 & 180 & 170 & 160 & 150 & 140 & 130 & 120 & 110 & 100 & 90 & 80 & 70 & 60 & 50 & 40 & 30 & 20 & 10 & 0 & -10\end{array}$

${ }^{13} \mathrm{C}$ NMR spectrum (125 MHz, $\mathrm{CDCl}_{3}$ ) of compound $\mathbf{6 e}$ 


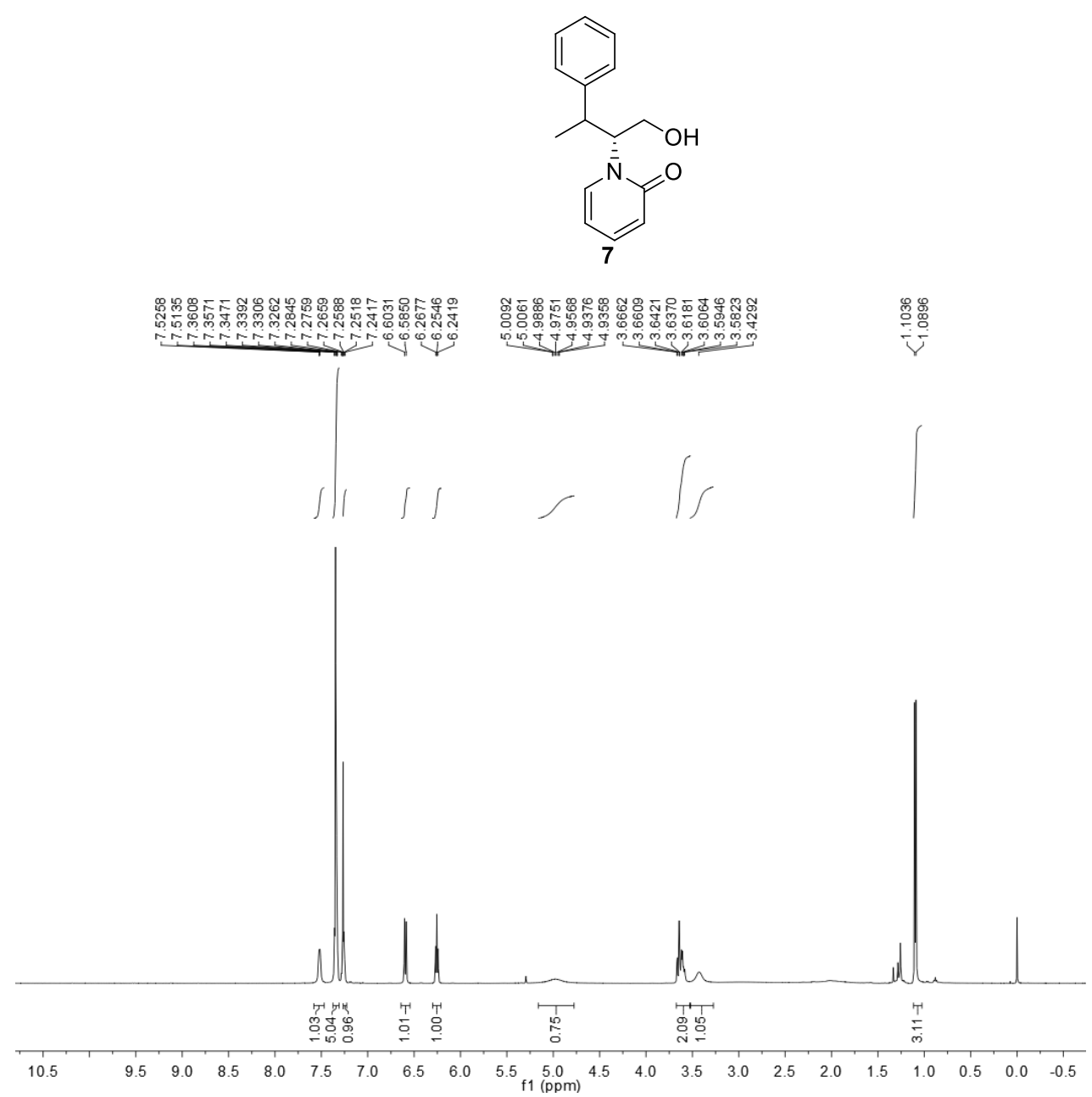

${ }^{1} \mathrm{H}$ NMR spectrum $\left(500 \mathrm{MHz}, \mathrm{CDCl}_{3}\right.$ ) of compound 7

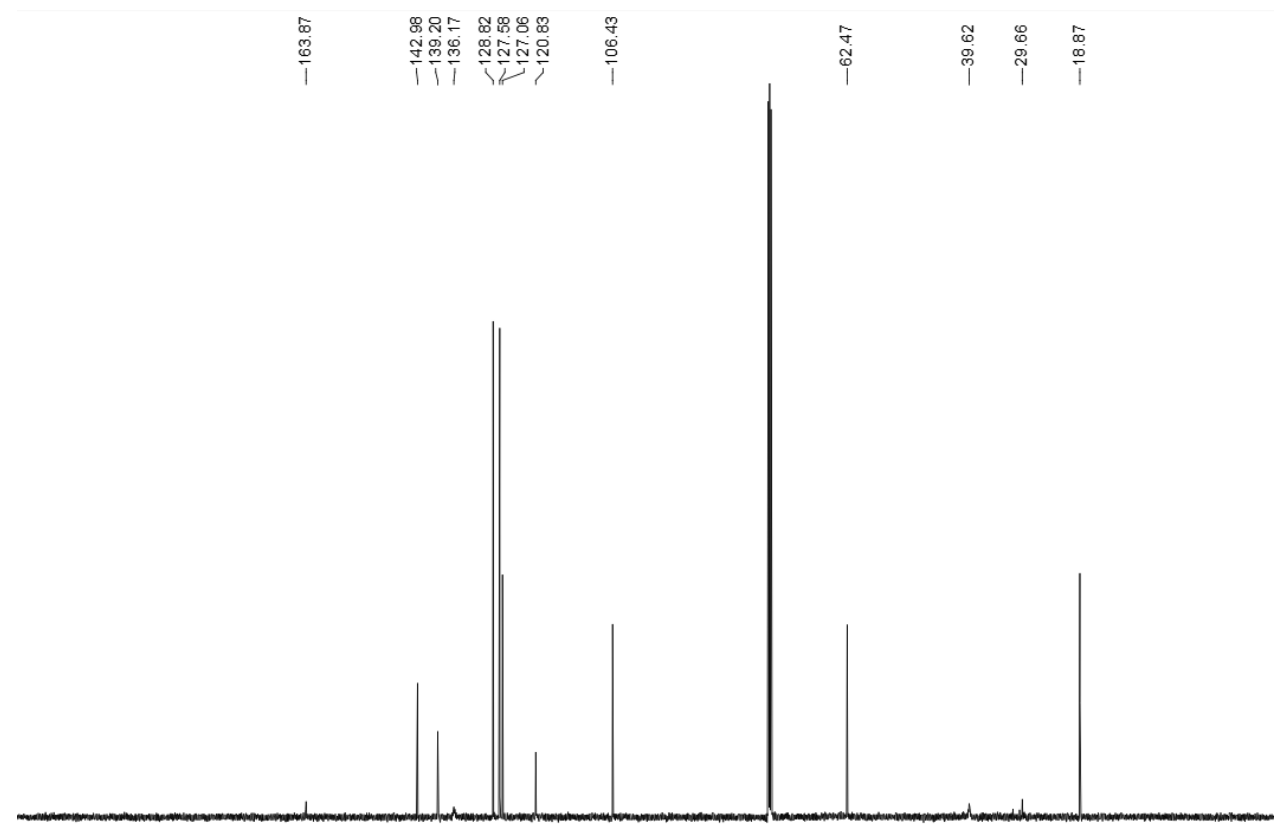

$\begin{array}{llllllllllllllllllllllll}210 & 200 & 190 & 180 & 170 & 160 & 150 & 140 & 130 & 120 & 110 & 100 & 90 & 80 & 70 & 60 & 50 & 40 & 30 & 20 & 10 & 0 & -10\end{array}$

${ }^{13} \mathrm{C}$ NMR spectrum $\left(125 \mathrm{MHz}, \mathrm{CDCl}_{3}\right.$ ) of compound 7 

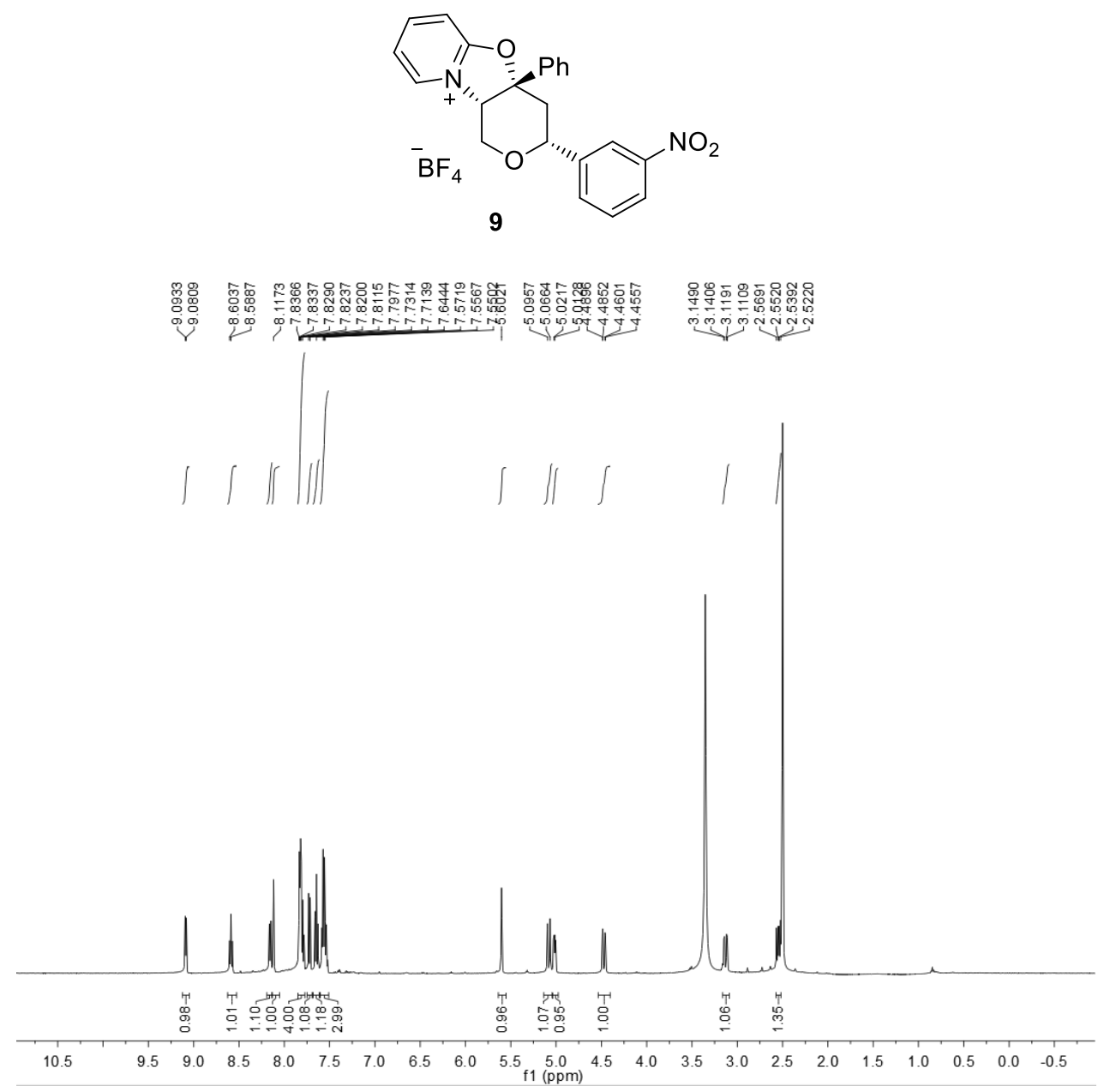

${ }^{1} \mathrm{H}$ NMR spectrum (500 MHz, DMSO) of compound 9
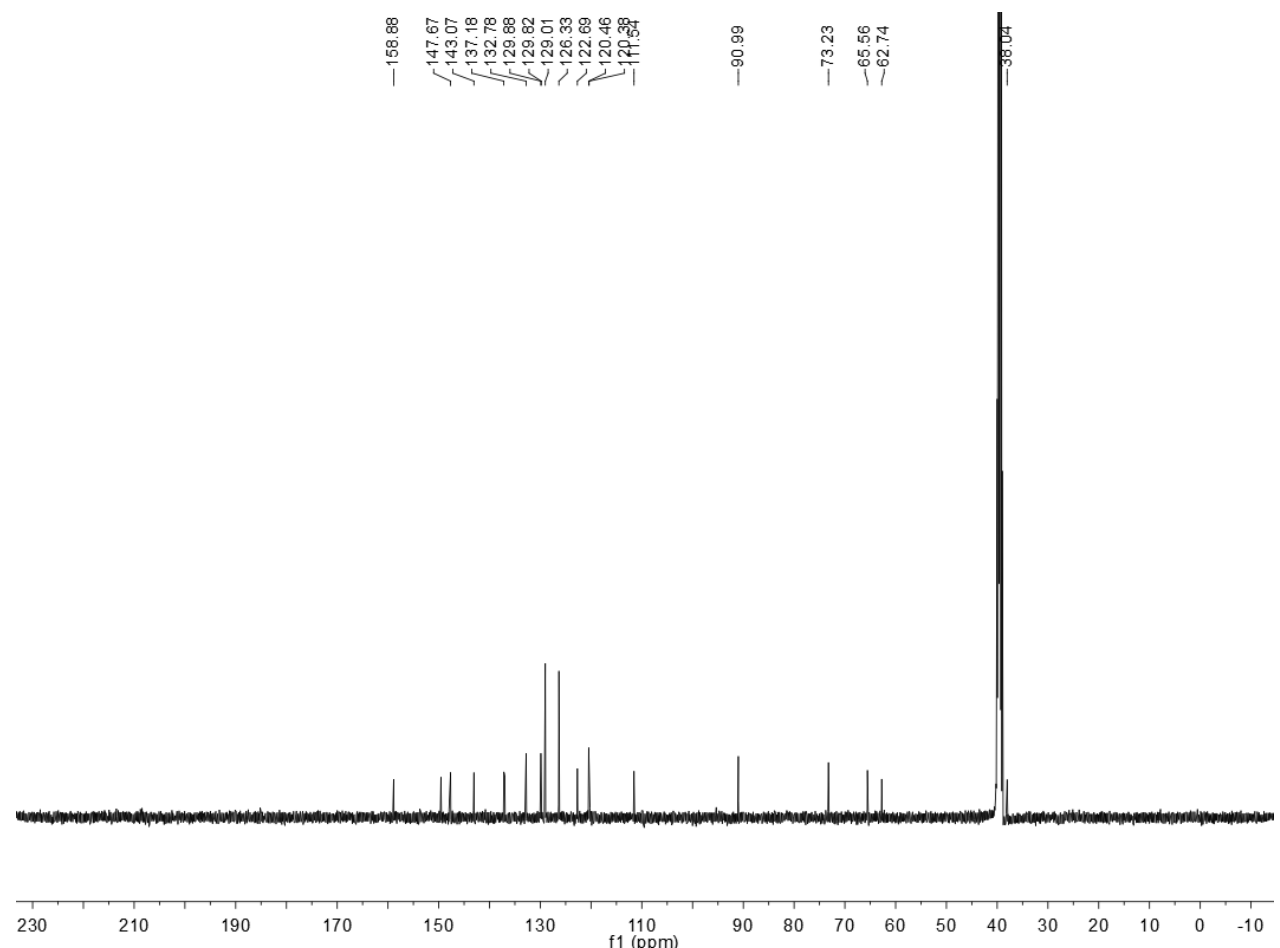

${ }^{13} \mathrm{C}$ NMR spectrum (125 MHz, DMSO) of compound 9 


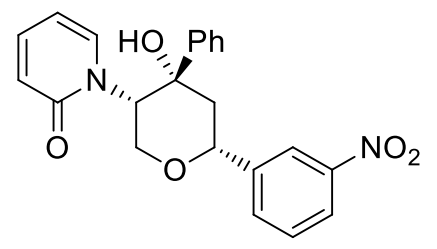

10
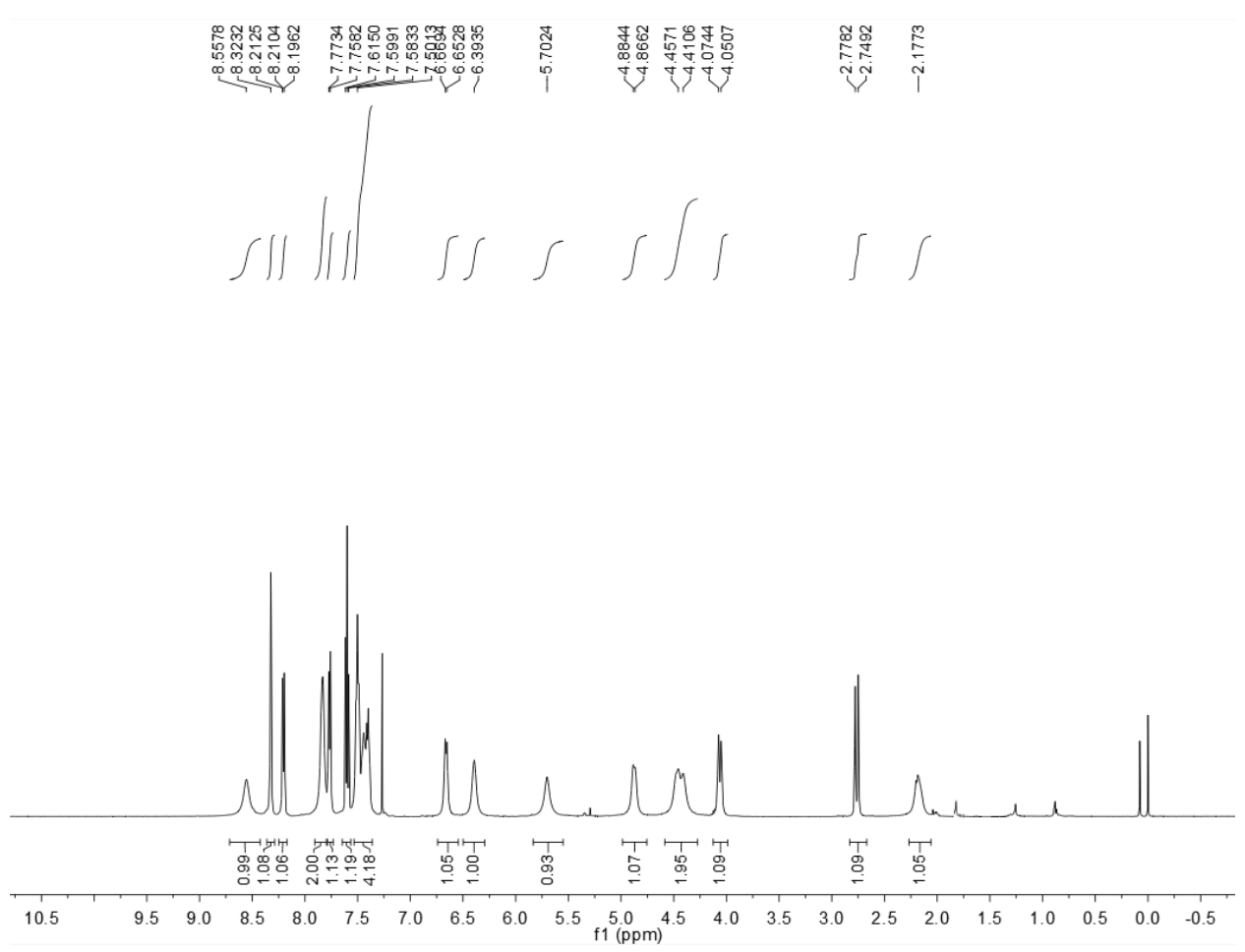

${ }^{1} \mathrm{H}$ NMR spectrum $\left(500 \mathrm{MHz}, \mathrm{CDCl}_{3}\right.$ ) of compound $\mathbf{1 0}$
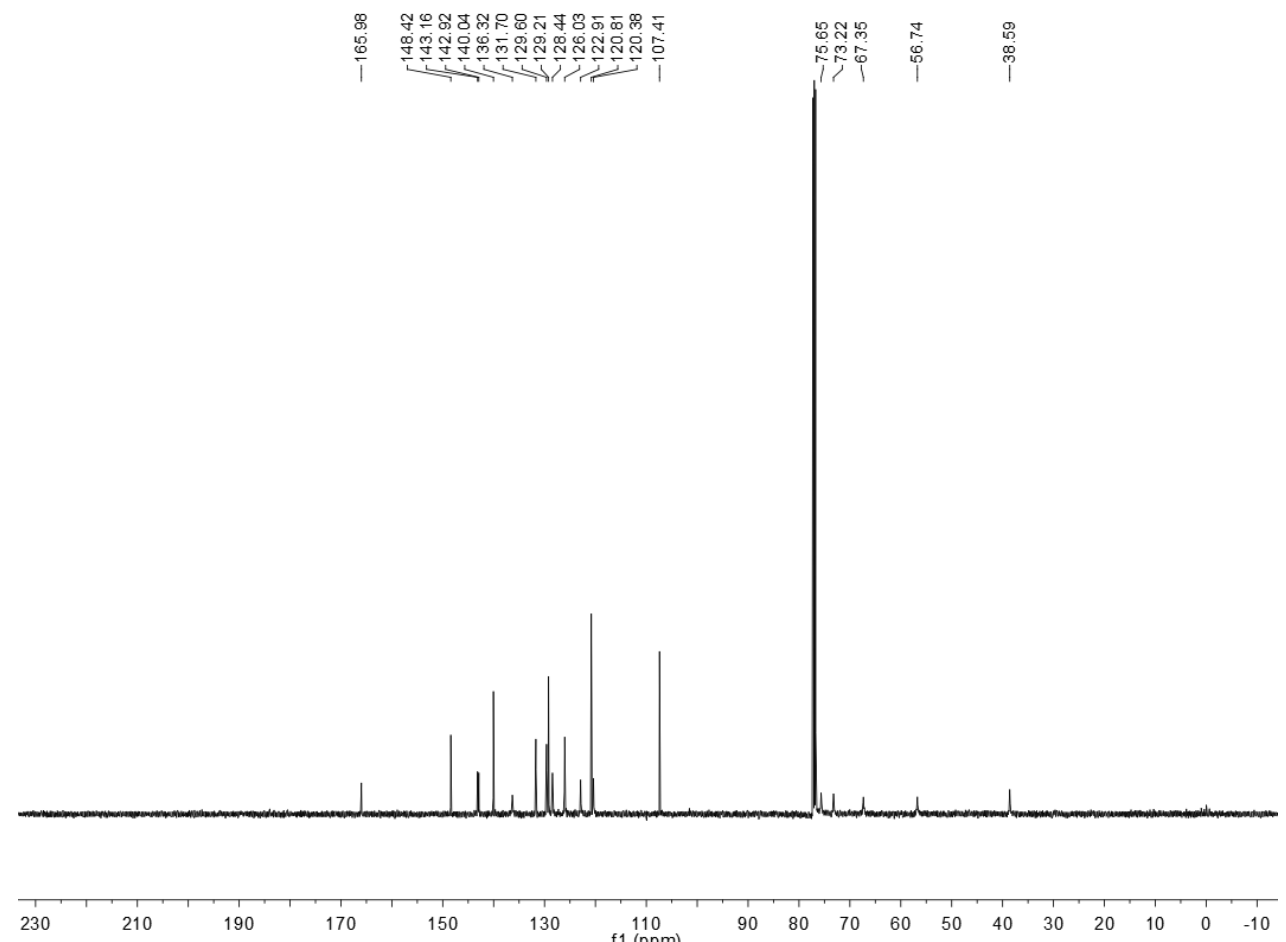

${ }^{13} \mathrm{C}$ NMR spectrum $\left(125 \mathrm{MHz}, \mathrm{CDCl}_{3}\right)$ of compound $\mathbf{1 0}$ 
HPLC charts

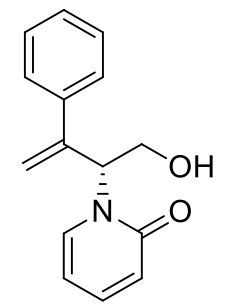

4aa
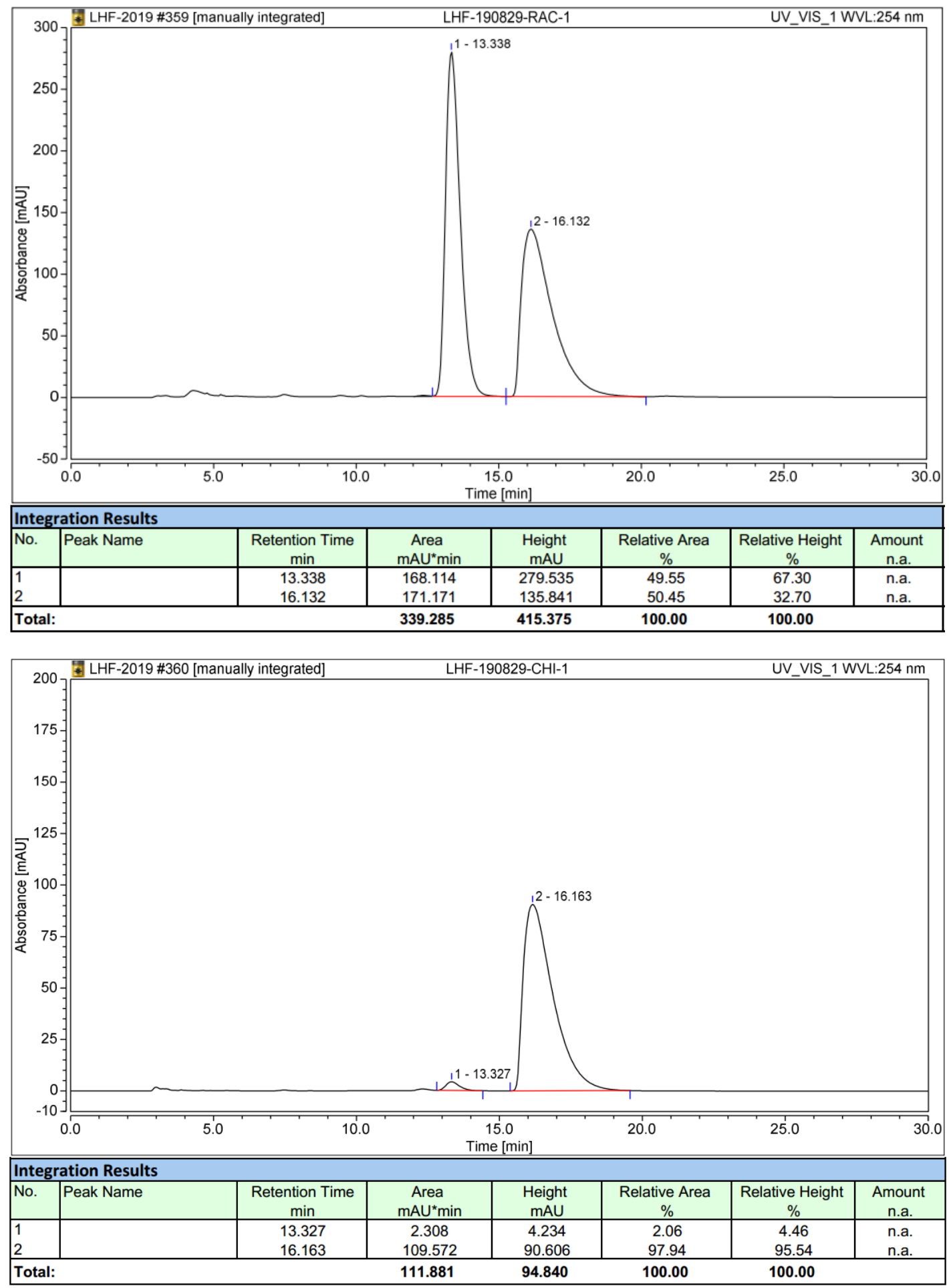

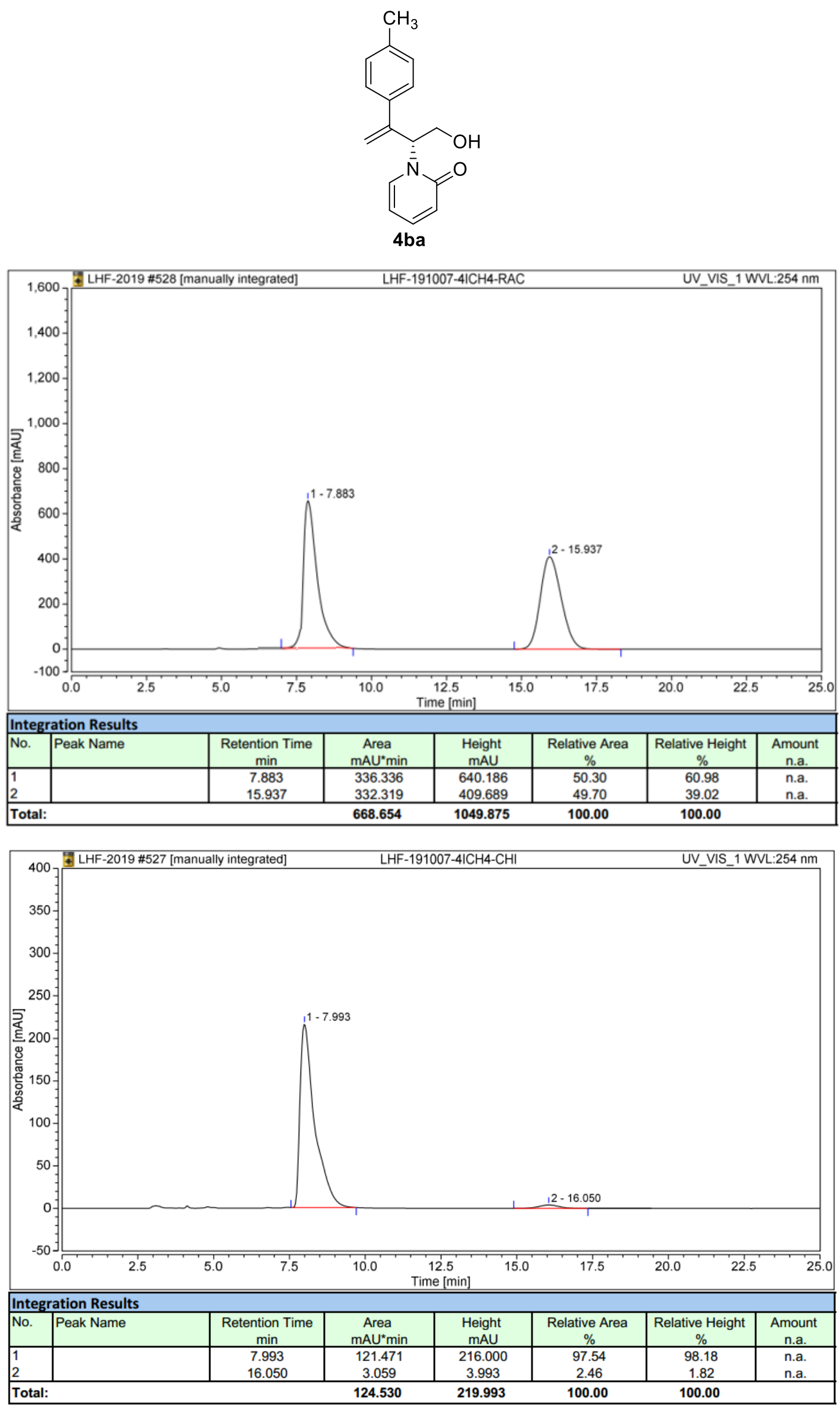

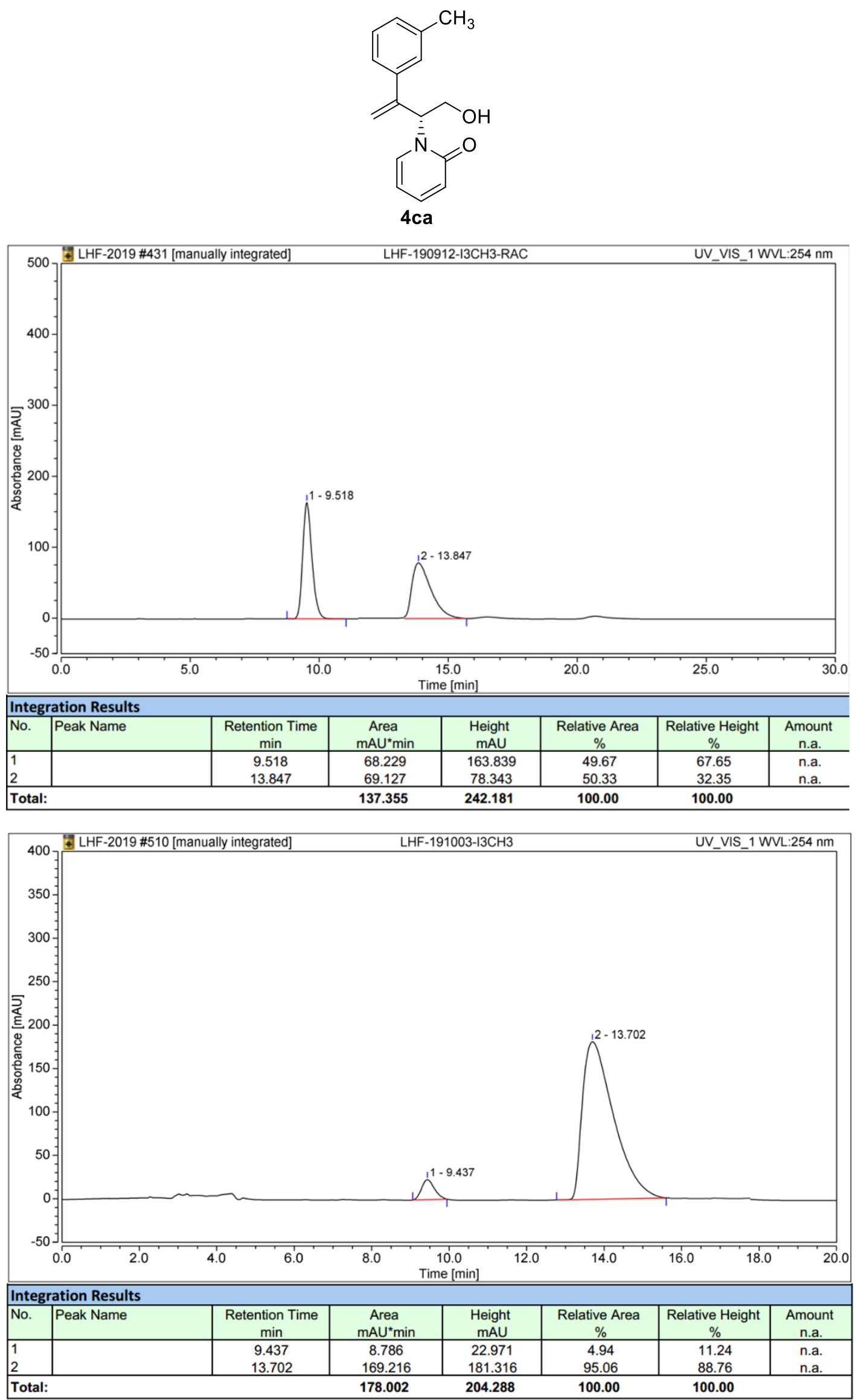


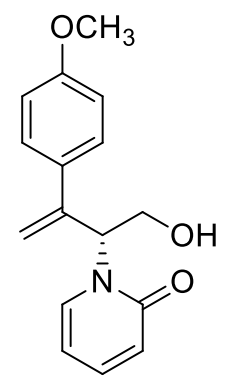

4da
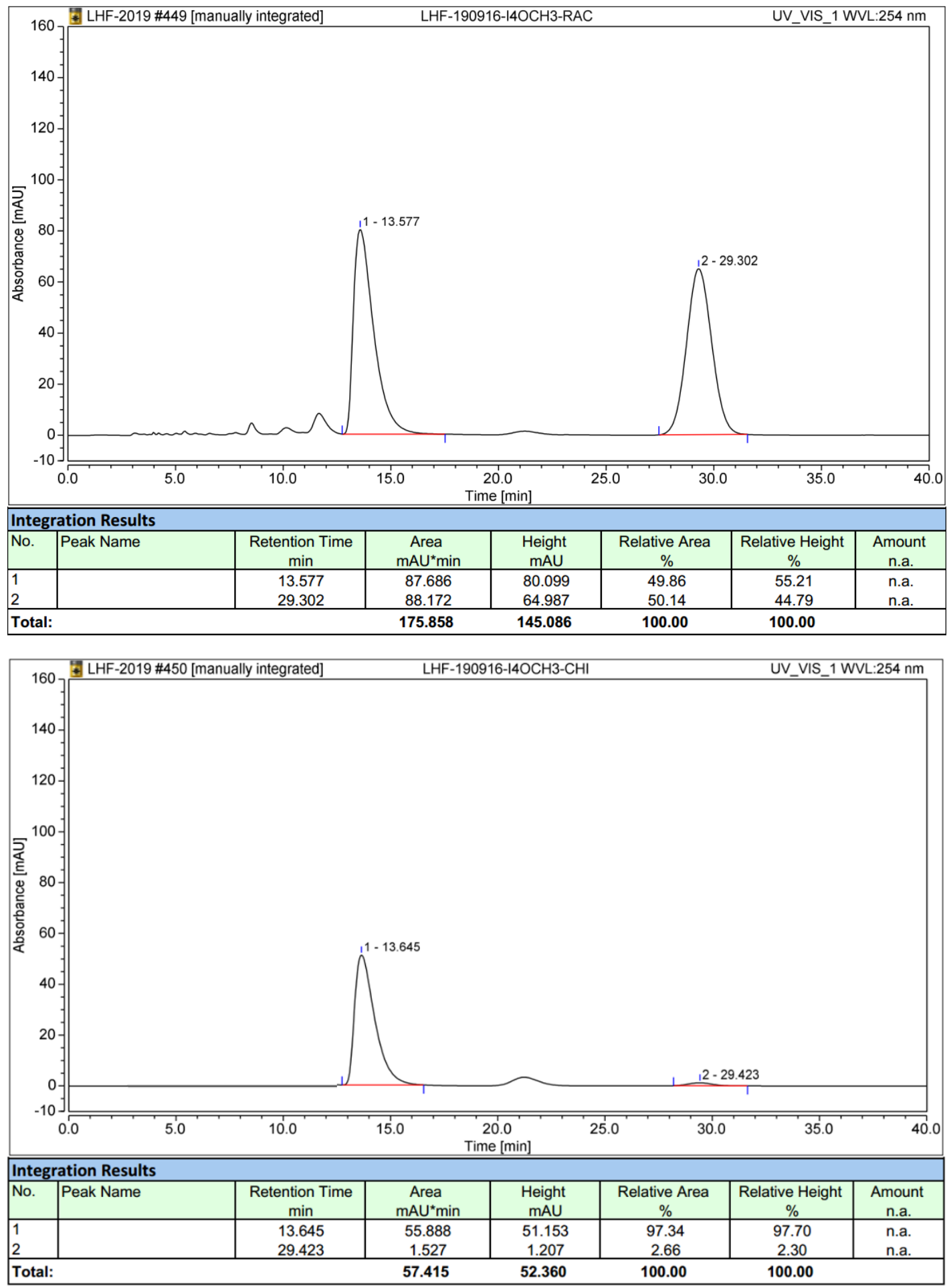

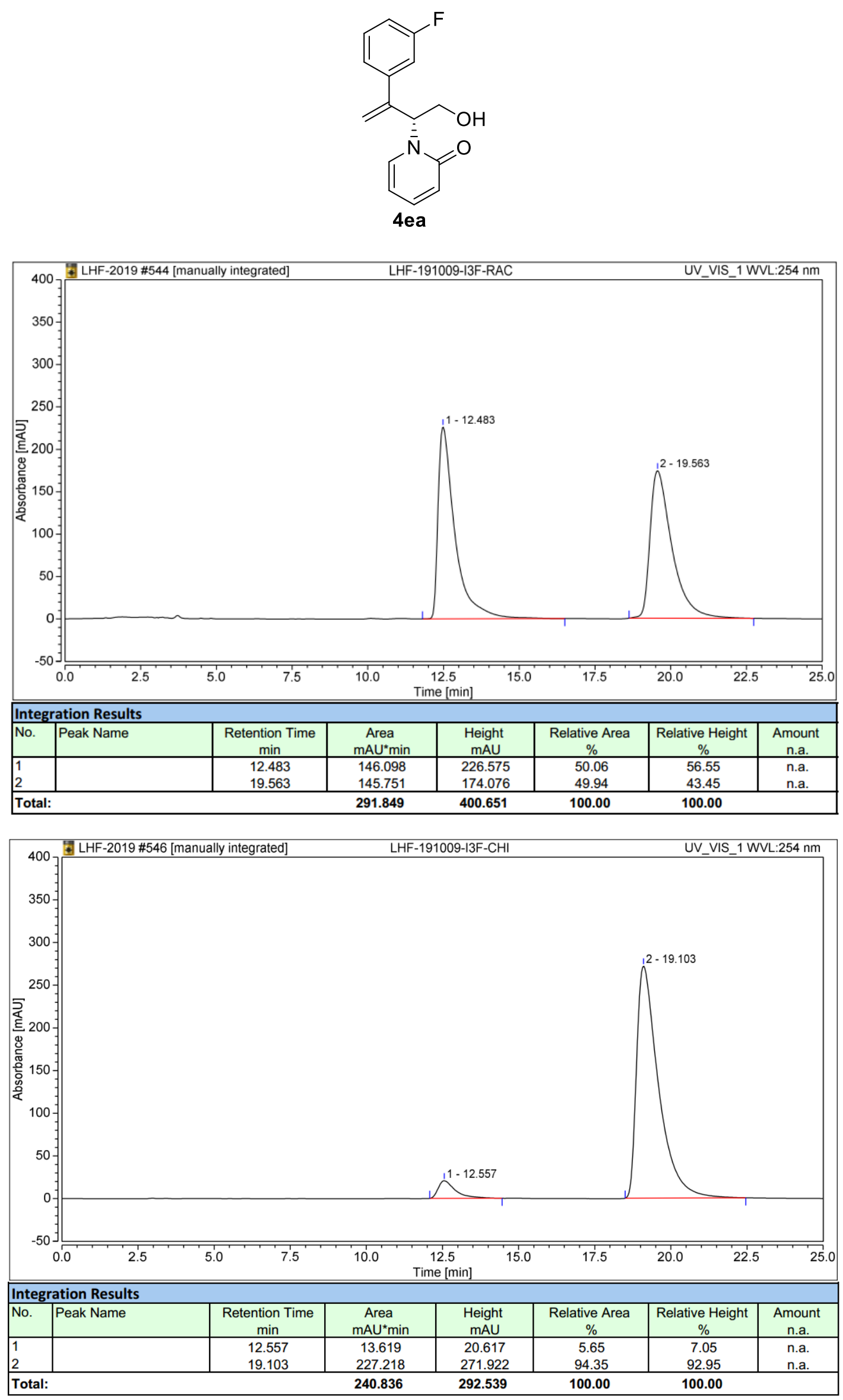

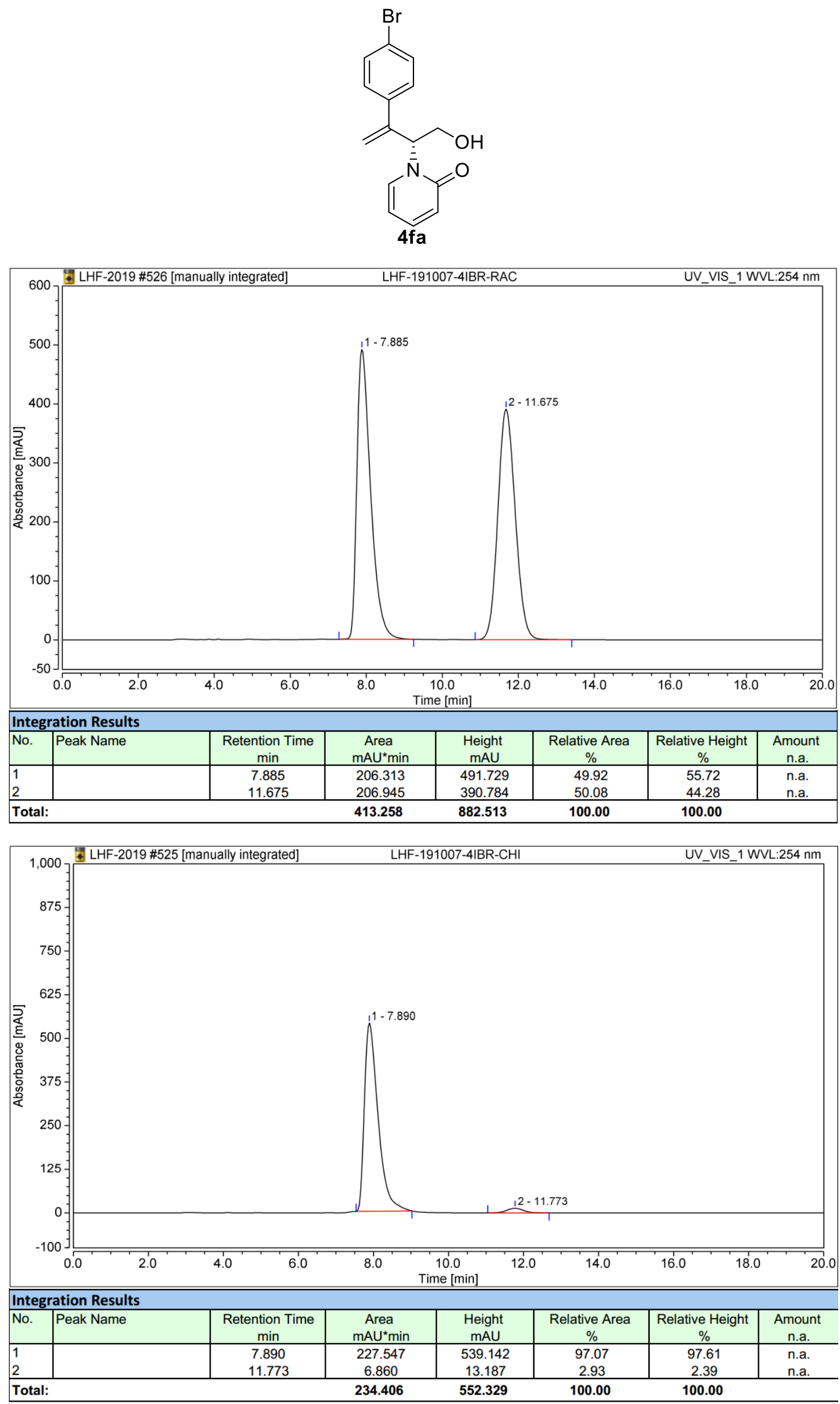

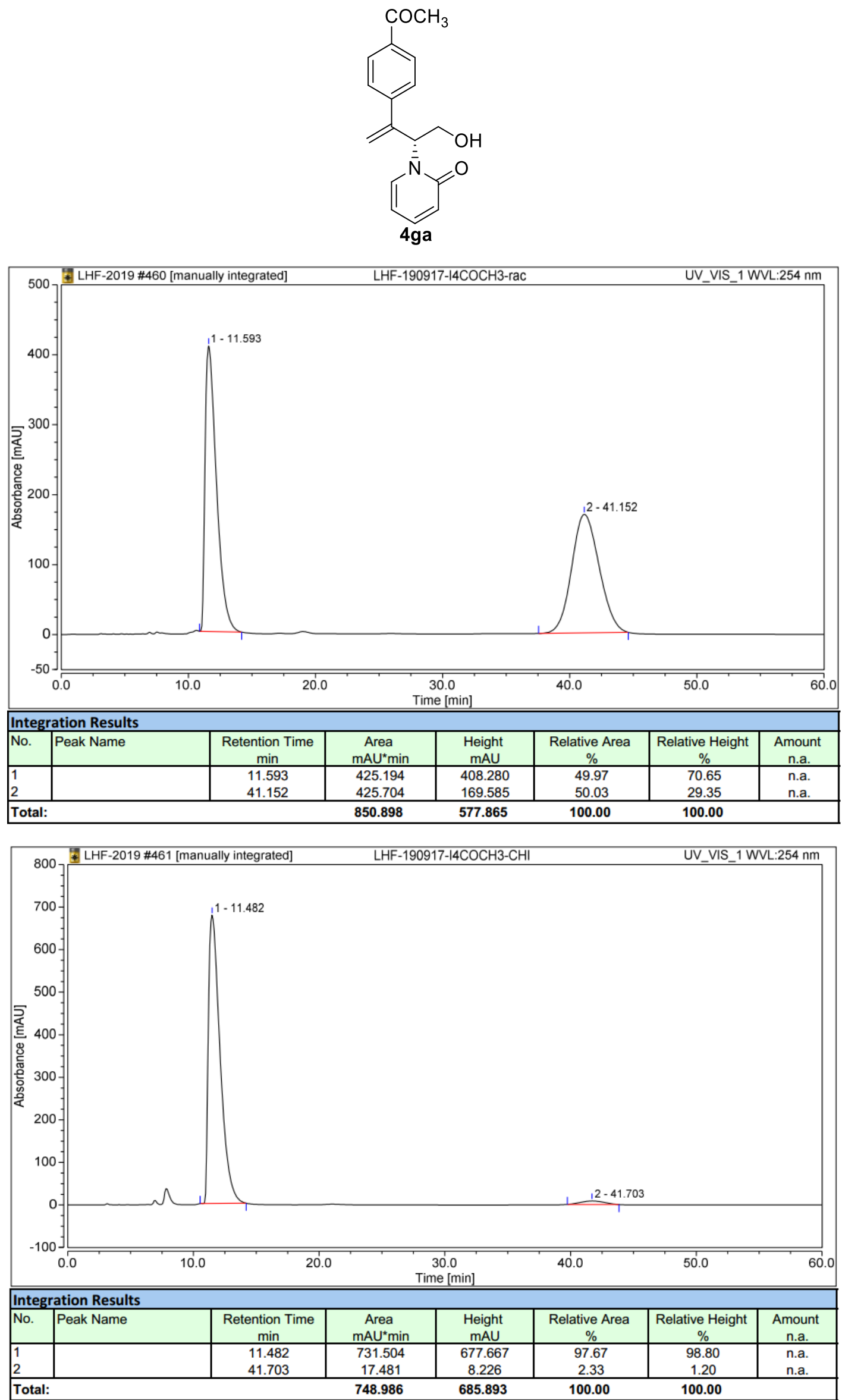

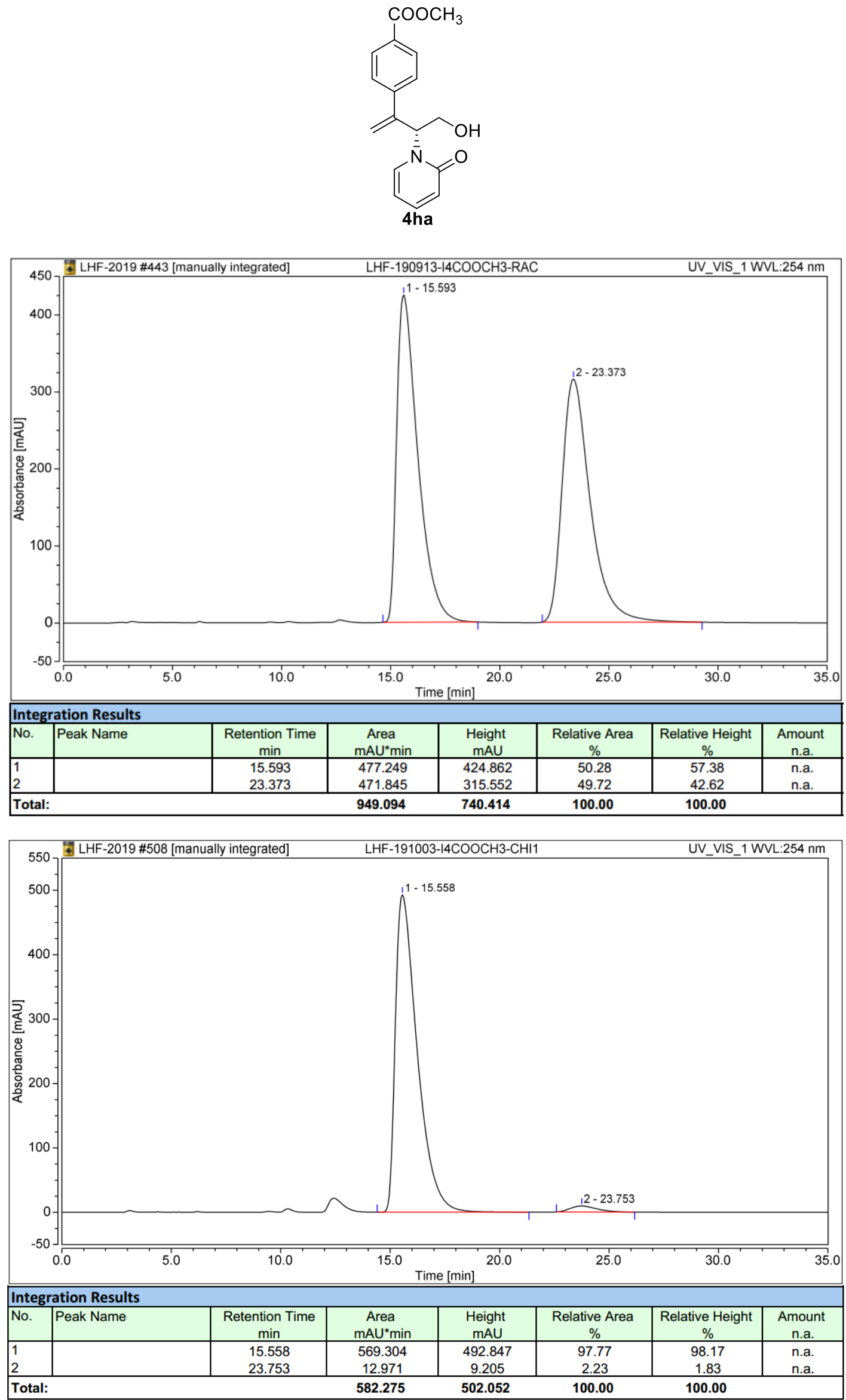

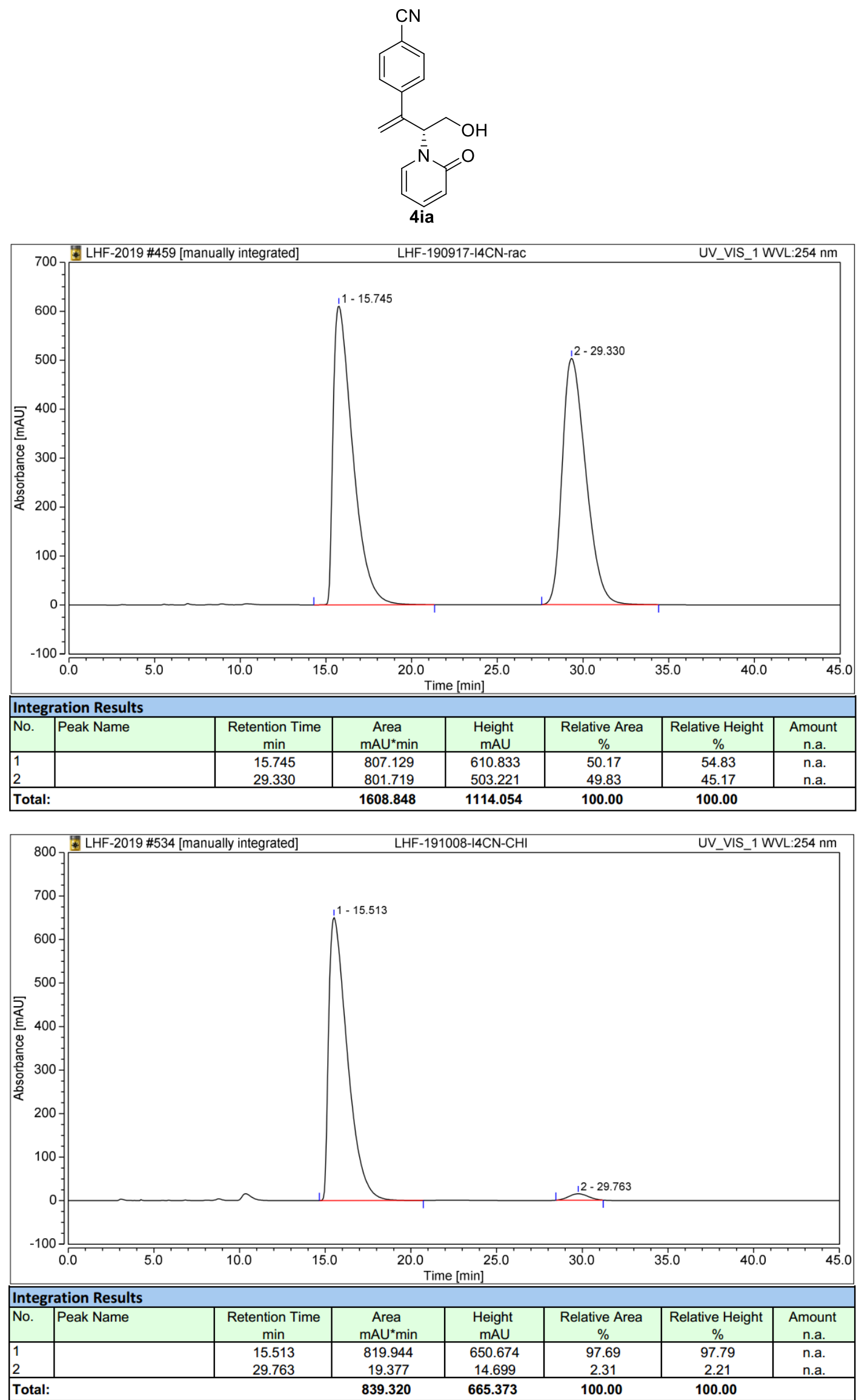

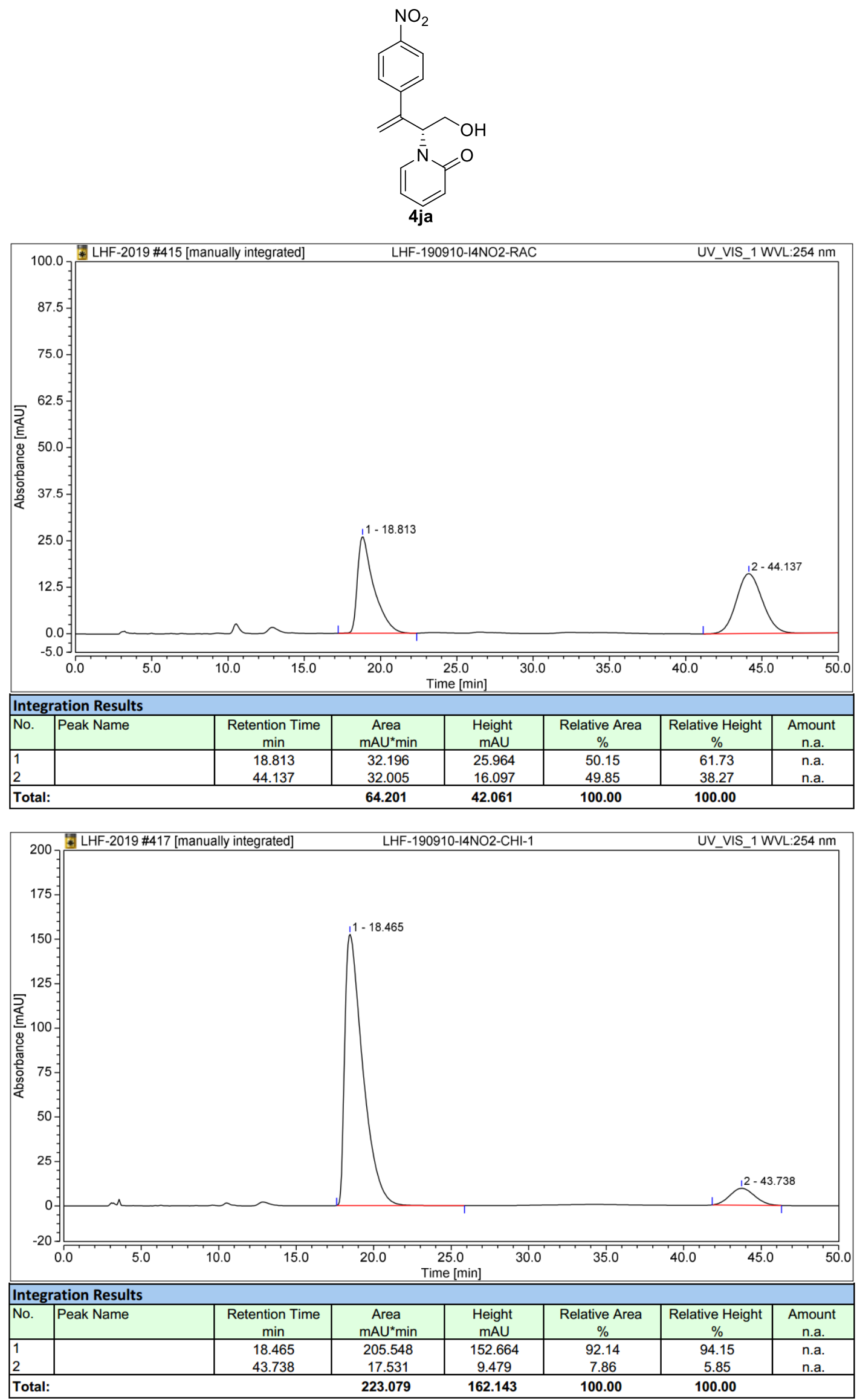

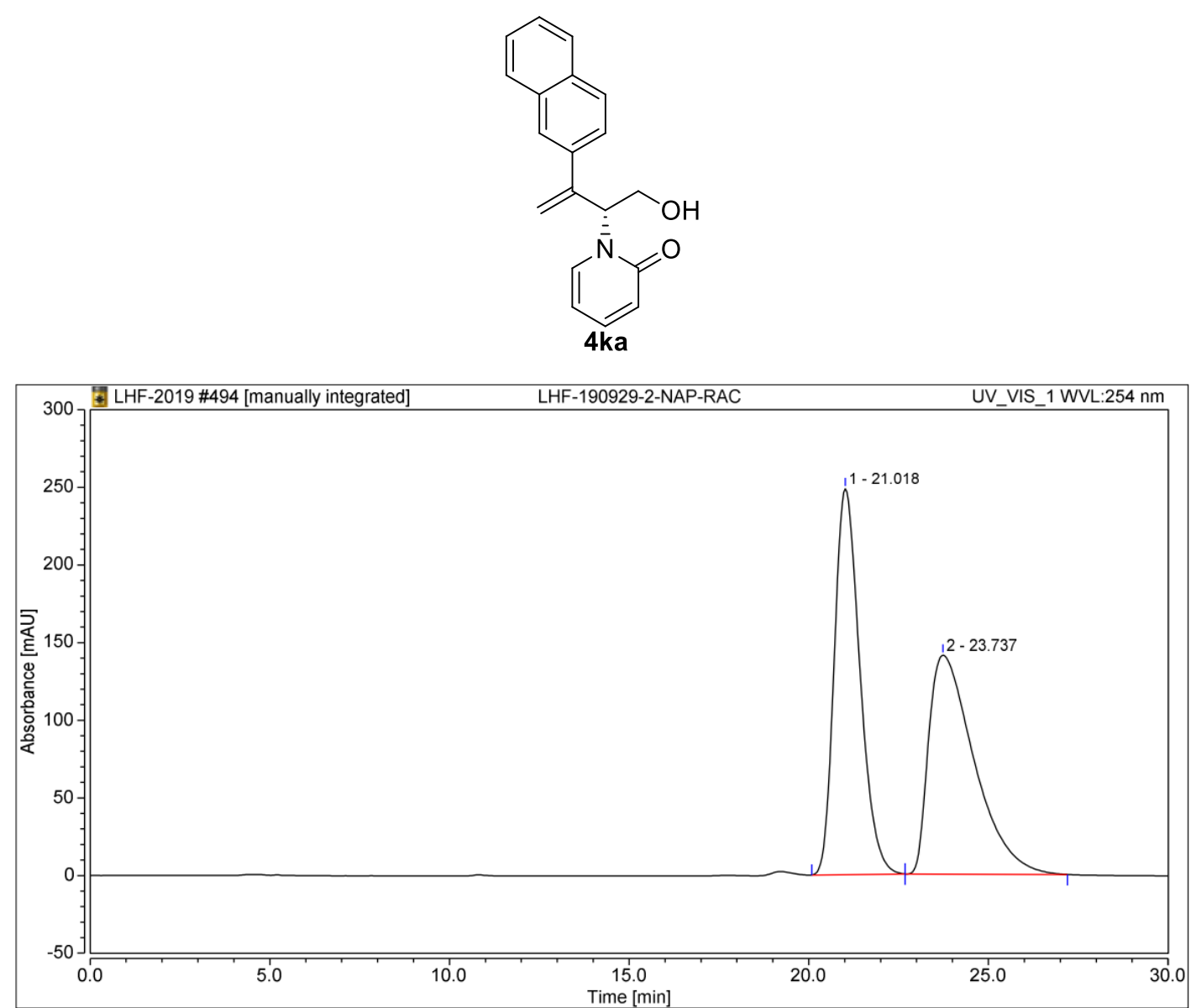

\begin{tabular}{|l|l|c|c|c|c|c|c|}
\hline \hline Integration Results \\
\hline No. & Peak Name & $\begin{array}{c}\text { Retention Time } \\
\text { min }\end{array}$ & $\begin{array}{c}\text { Area } \\
\text { mAU*min }\end{array}$ & $\begin{array}{c}\text { Height } \\
\text { mAU }\end{array}$ & $\begin{array}{c}\text { Relative Area } \\
\%\end{array}$ & $\begin{array}{c}\text { Relative Height } \\
\%\end{array}$ & $\begin{array}{c}\text { Amount } \\
\text { n.a. }\end{array}$ \\
\hline 1 & 21.018 & 204.637 & 248.571 & 49.70 & 63.81 & n.a. \\
2 & 23.737 & 207.117 & 140.957 & 50.30 & 36.19 & n.a. \\
\hline
\end{tabular}

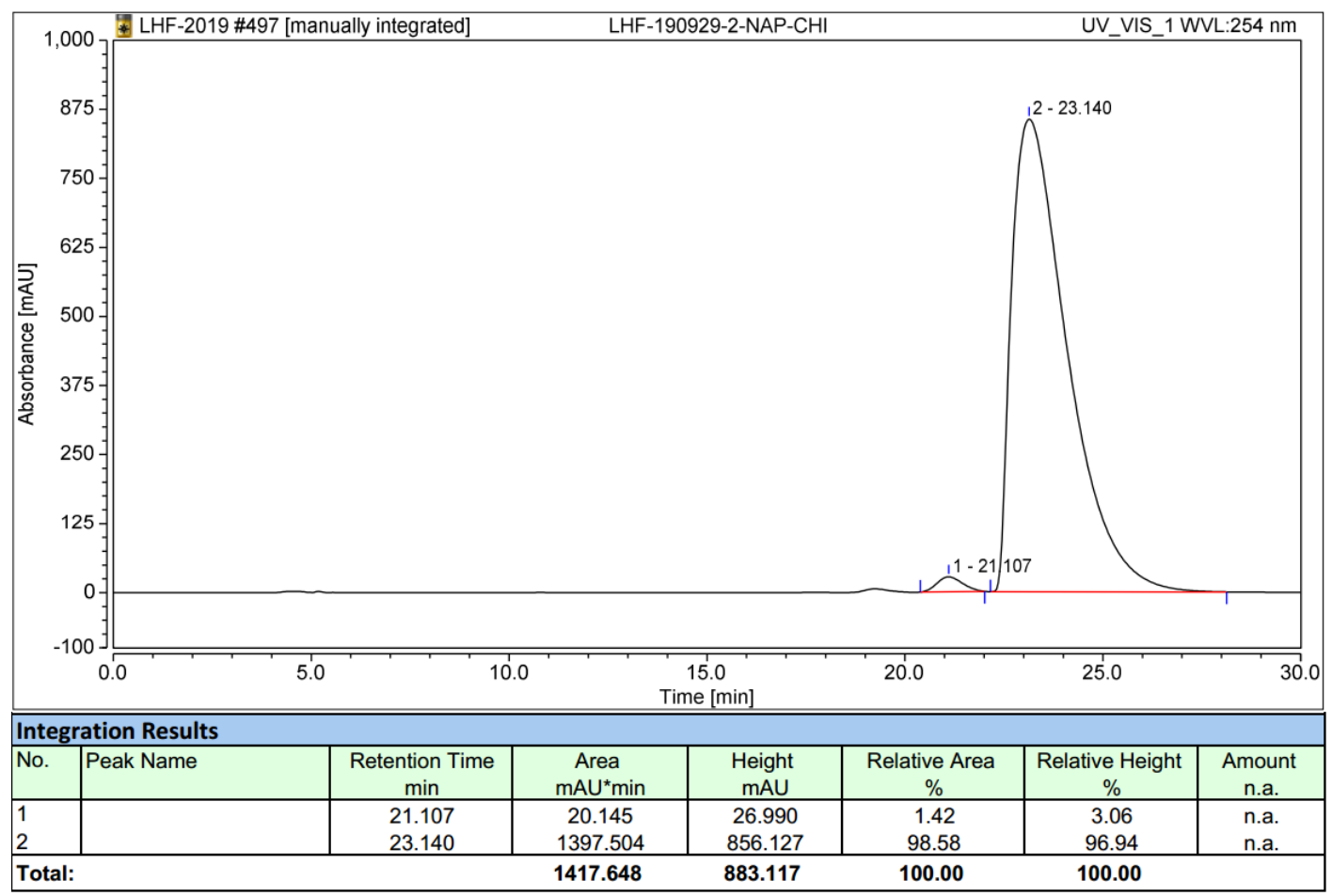



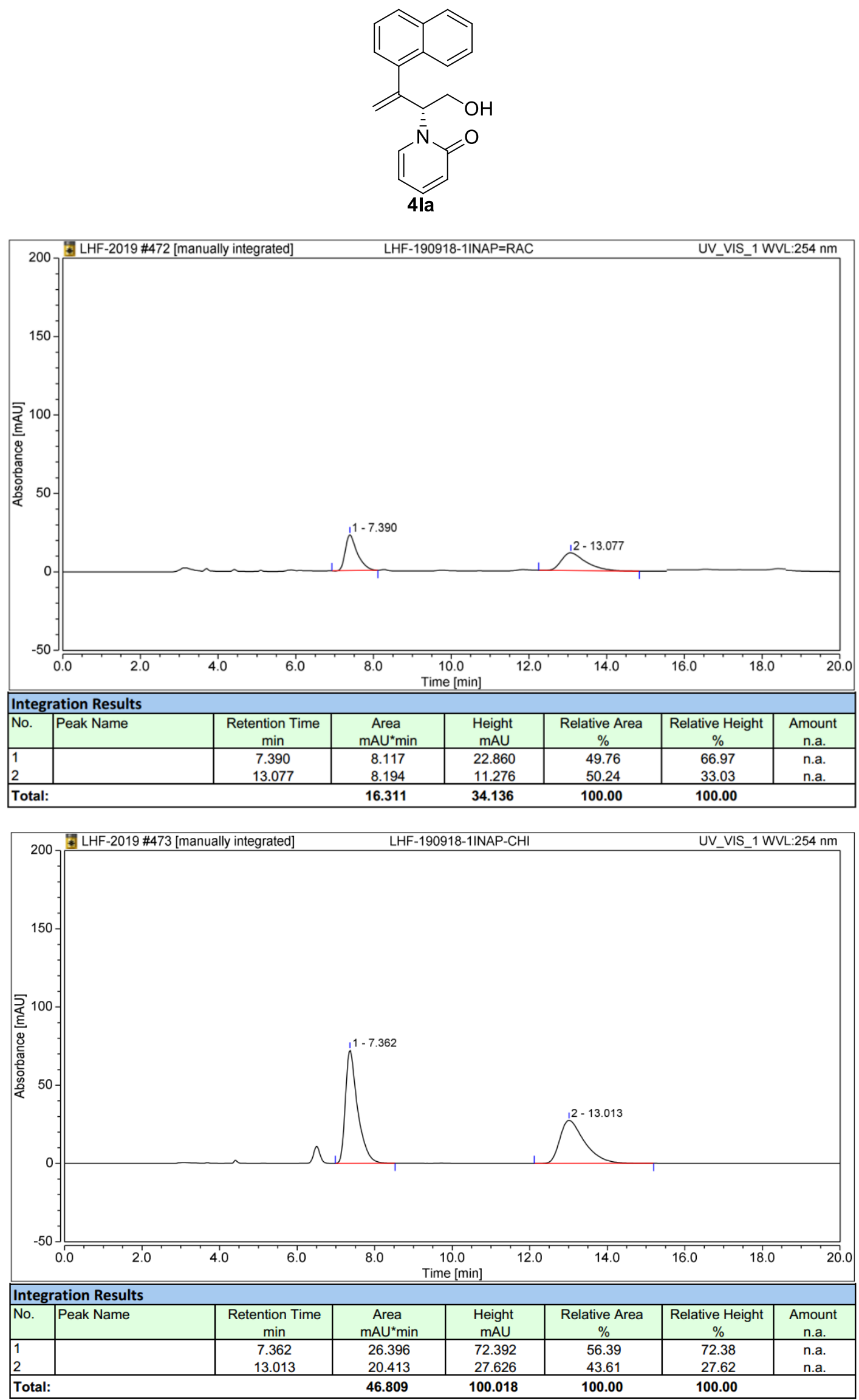
<smiles>C=C(c1ccccc1)C(CO)n1cc(C)cc(C)c1=O</smiles>
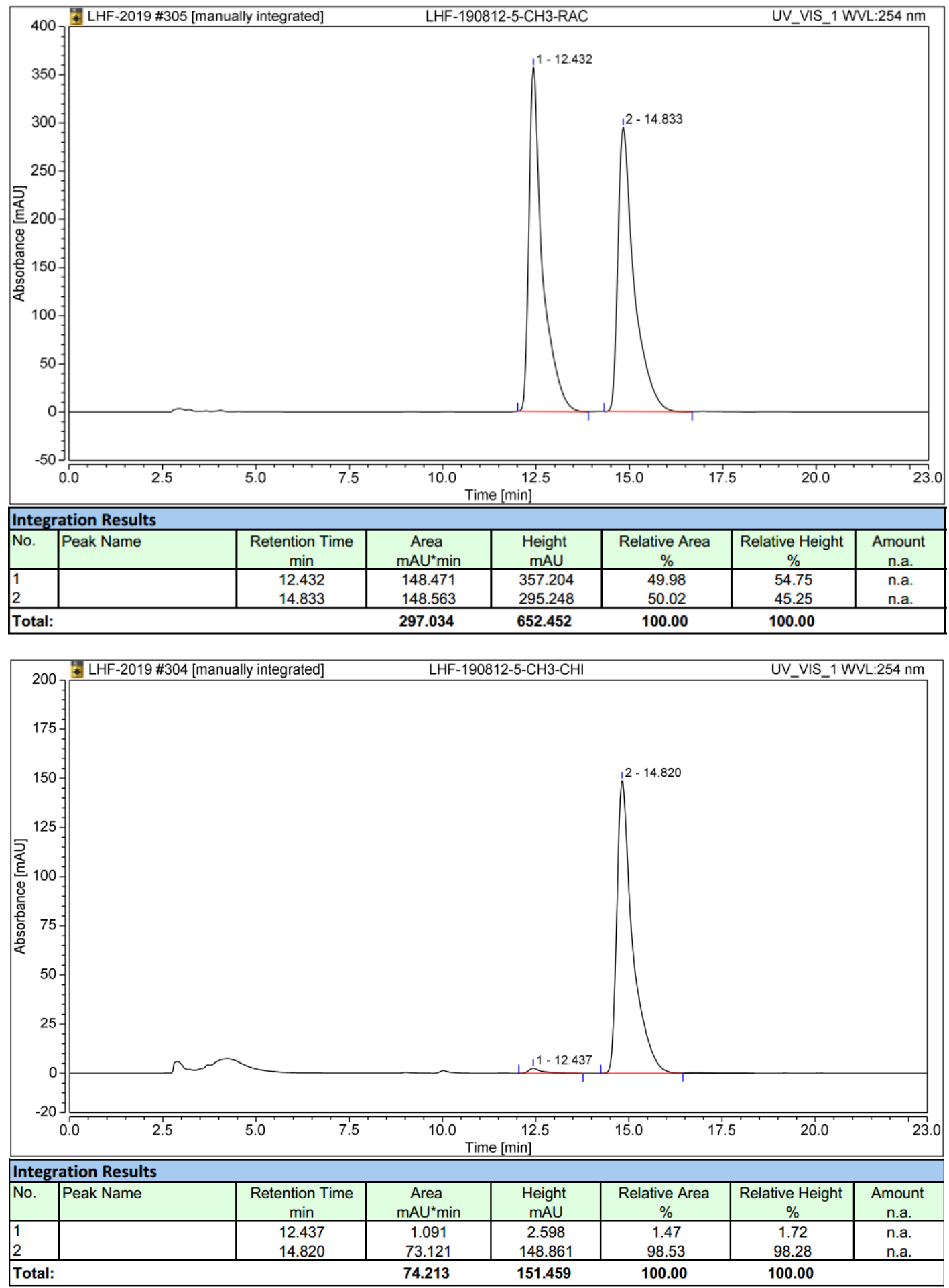


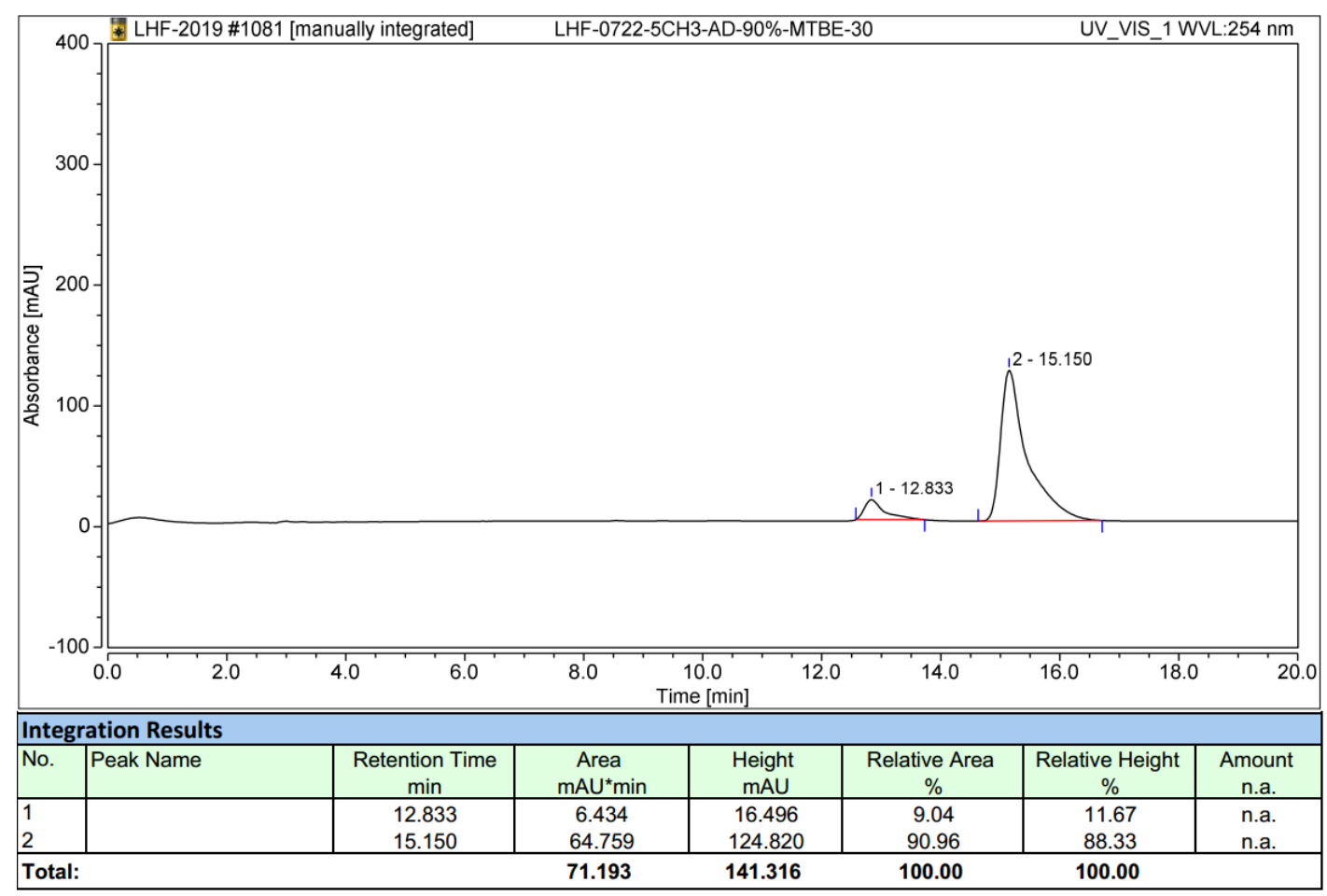<smiles>C=C(c1ccccc1)[C@H](CO)n1ccc(C(C)(C)C)cc1=O</smiles>

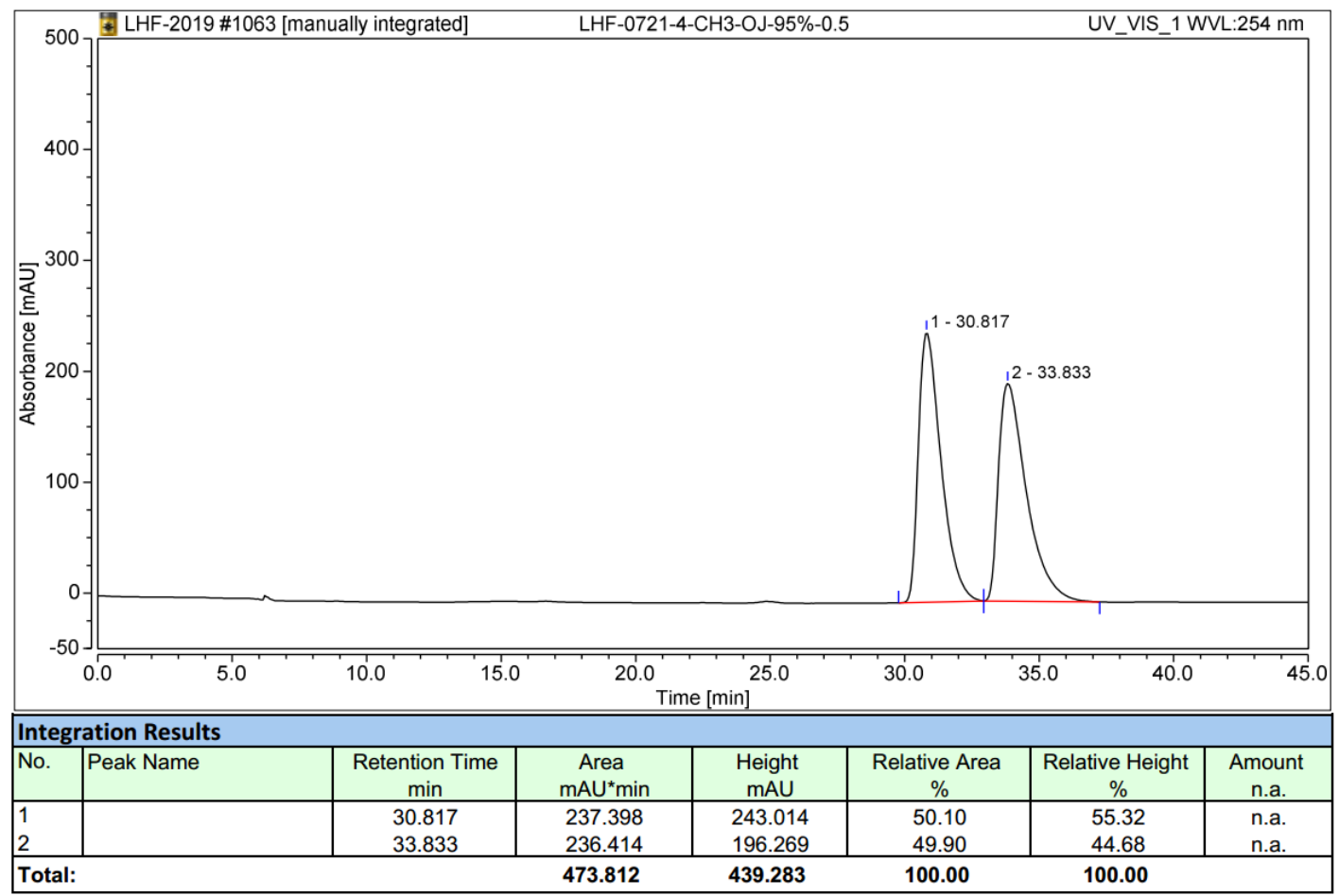




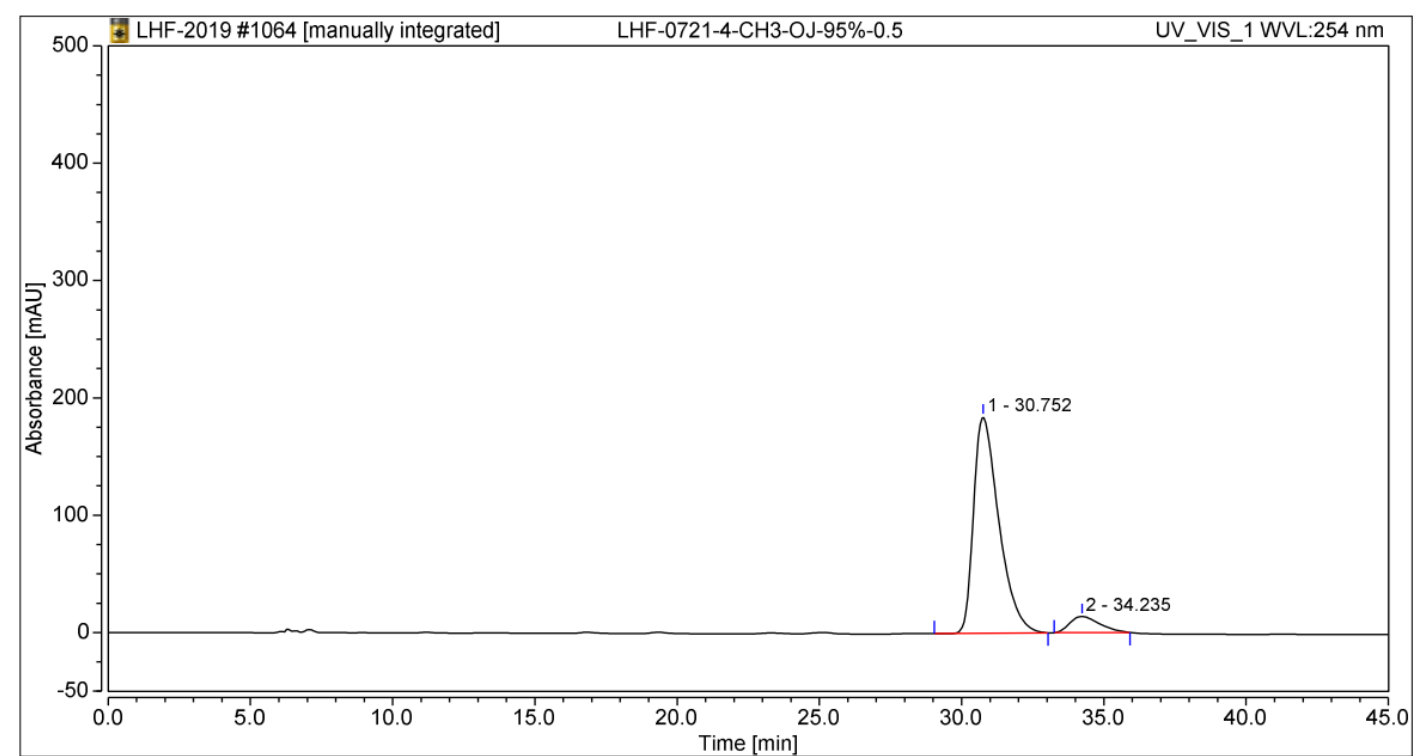

\begin{tabular}{|c|c|c|c|c|c|c|c|}
\hline \multicolumn{8}{|c|}{ Integration Results } \\
\hline No. & Peak Name & $\begin{array}{c}\text { Retention Time } \\
\text { min }\end{array}$ & $\begin{array}{c}\text { Area } \\
\mathrm{mAU^{* } \operatorname { m i n }}\end{array}$ & $\begin{array}{c}\text { Height } \\
\text { mAU }\end{array}$ & $\begin{array}{c}\text { Relative Area } \\
\% \\
\end{array}$ & $\begin{array}{c}\text { Relative Height } \\
\%\end{array}$ & $\begin{array}{c}\text { Amount } \\
\text { n.a. }\end{array}$ \\
\hline $\begin{array}{l}1 \\
2 \\
\end{array}$ & & $\begin{array}{l}30.752 \\
34.235 \\
\end{array}$ & $\begin{array}{c}190.155 \\
16.791 \\
\end{array}$ & $\begin{array}{c}184.322 \\
13.855 \\
\end{array}$ & $\begin{array}{c}91.89 \\
8.11 \\
\end{array}$ & $\begin{array}{c}93.01 \\
6.99 \\
\end{array}$ & $\begin{array}{l}\text { n.a. } \\
\text { n.a. }\end{array}$ \\
\hline \multicolumn{3}{|c|}{ Total: } & 206.946 & 198.177 & 100.00 & 100.00 & \\
\hline
\end{tabular}

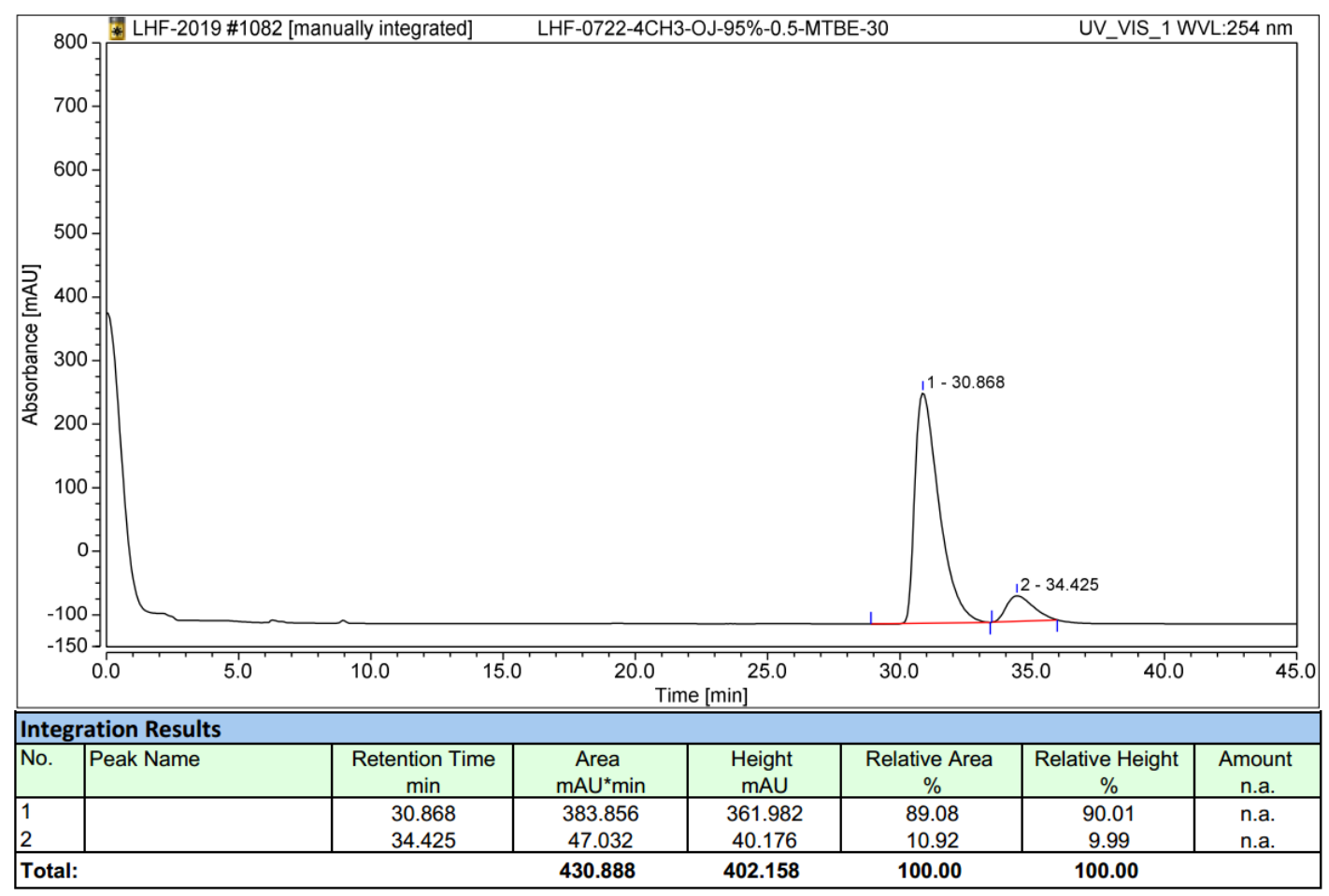<smiles>C=C(c1ccccc1)[C@H](CO)n1cccc([14CH3])c1=O</smiles> 


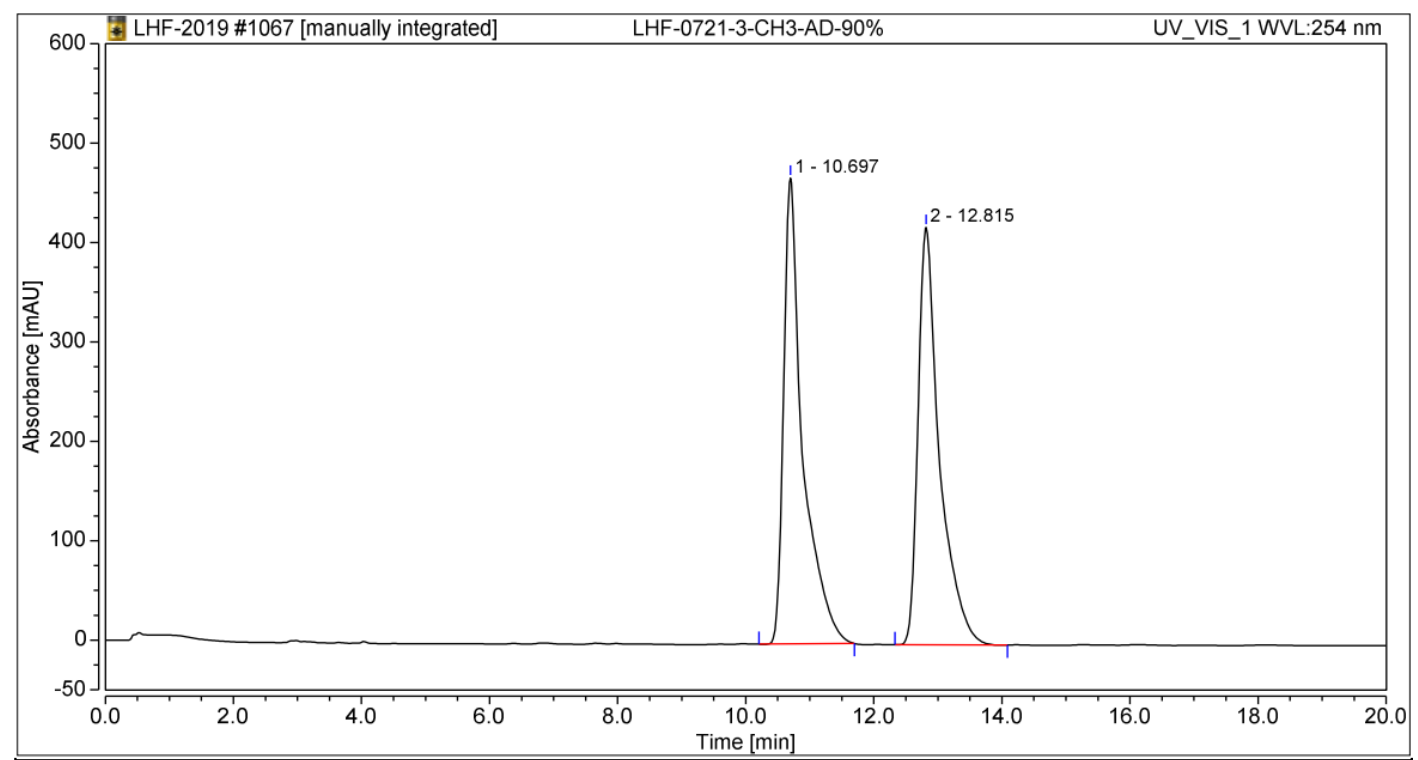

\begin{tabular}{|c|c|c|c|c|c|c|c|}
\hline \multicolumn{8}{|c|}{ Integration Results } \\
\hline No. & Peak Name & $\begin{array}{c}\text { Retention Time } \\
\text { min }\end{array}$ & $\begin{array}{c}\text { Area } \\
\mathrm{mAU}^{*} \min \end{array}$ & $\begin{array}{c}\text { Height } \\
\text { mAU }\end{array}$ & $\begin{array}{c}\text { Relative Area } \\
\% \\
\end{array}$ & $\begin{array}{c}\text { Relative Height } \\
\% \\
\end{array}$ & $\begin{array}{c}\text { Amount } \\
\text { n.a. }\end{array}$ \\
\hline $\begin{array}{l}1 \\
2\end{array}$ & & $\begin{array}{l}10.697 \\
12.815\end{array}$ & $\begin{array}{l}161.916 \\
162.661\end{array}$ & $\begin{array}{l}468.679 \\
419.898\end{array}$ & $\begin{array}{l}49.89 \\
50.11\end{array}$ & $\begin{array}{l}52.74 \\
47.26\end{array}$ & $\begin{array}{l}\text { n.a. } \\
\text { n.a. }\end{array}$ \\
\hline \multicolumn{3}{|c|}{ Total: } & 324.577 & 888.577 & 100.00 & 100.00 & \\
\hline
\end{tabular}

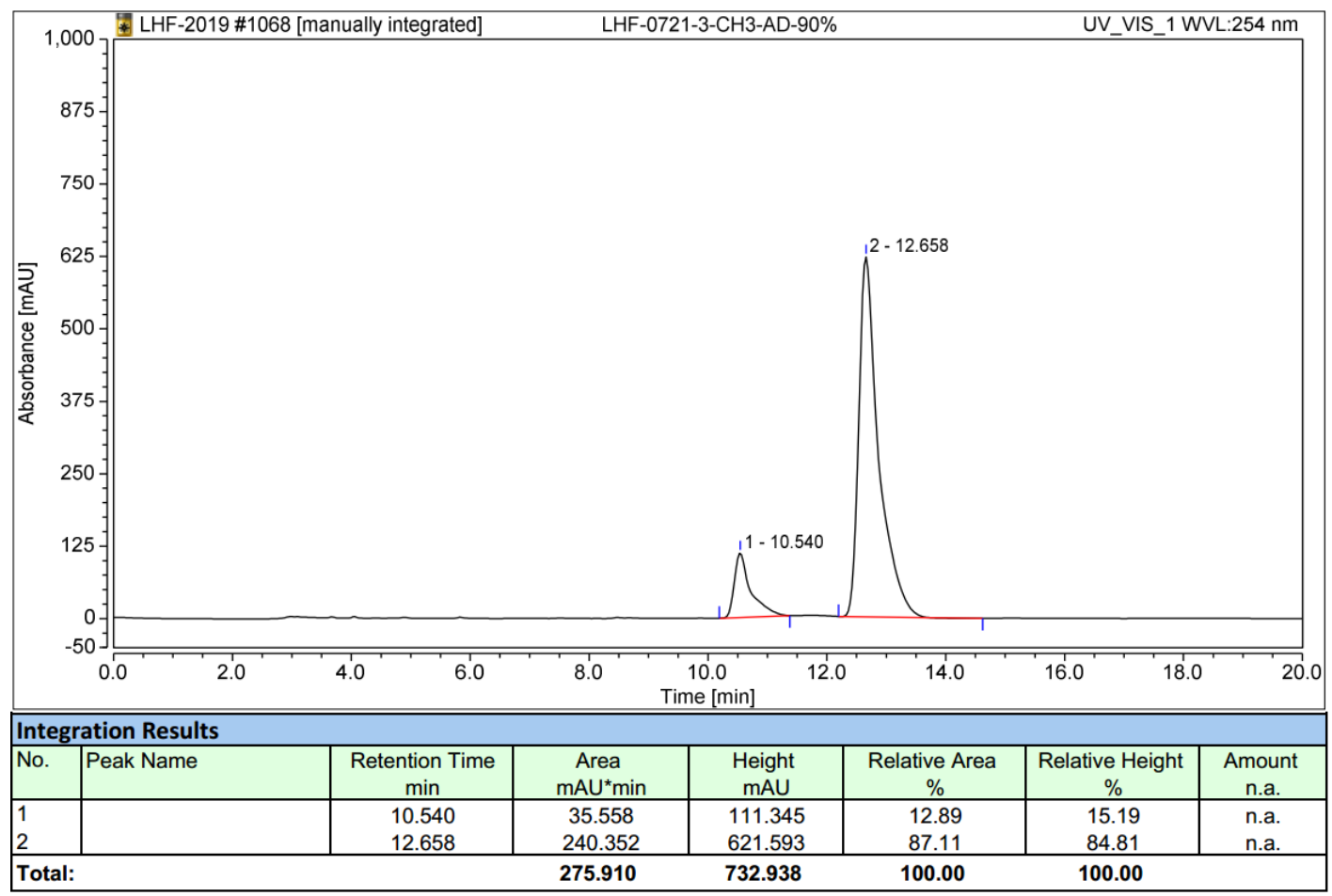




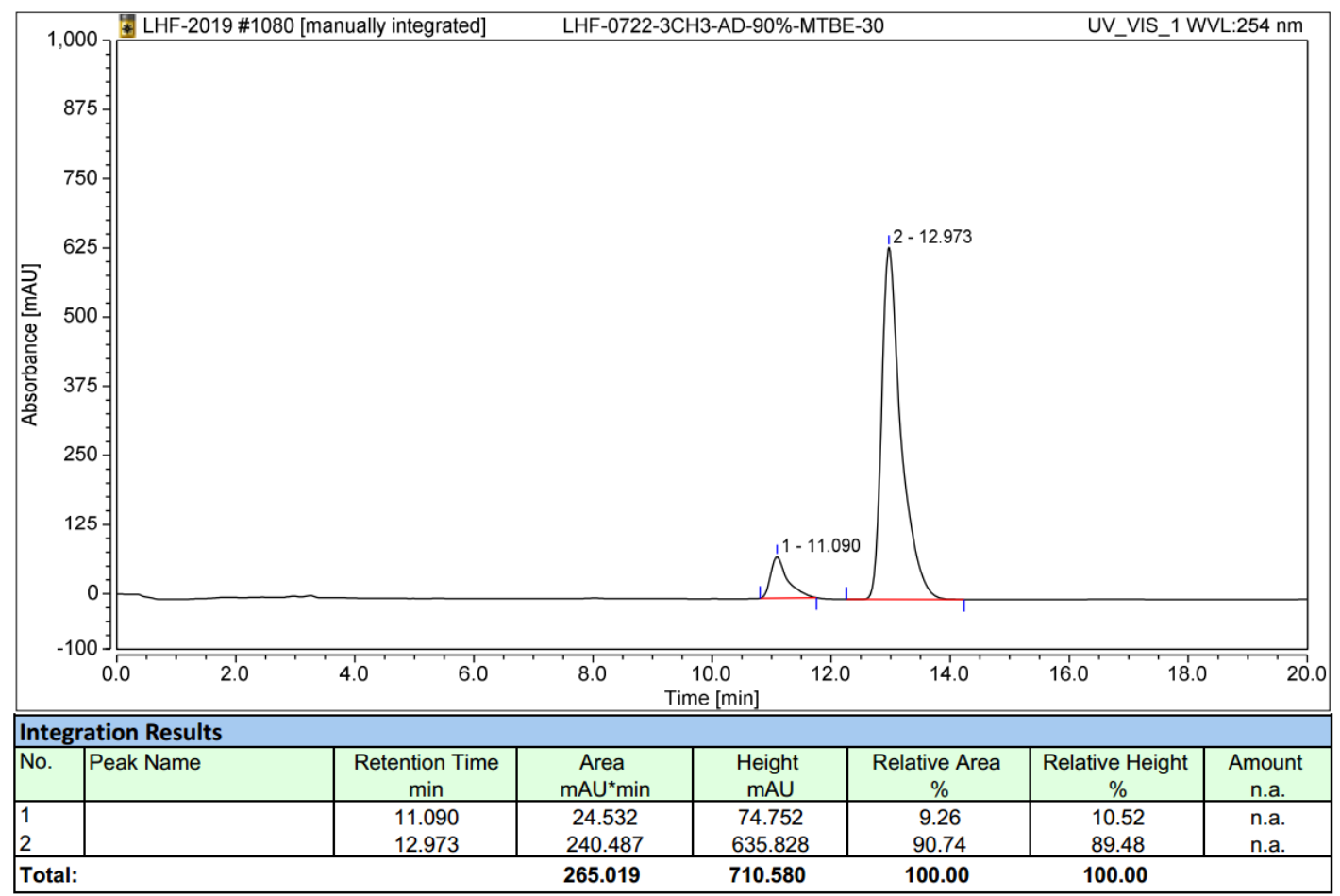<smiles>C=C(c1ccccc1)[C@H](CO)n1ccc(Cl)cc1=O</smiles>

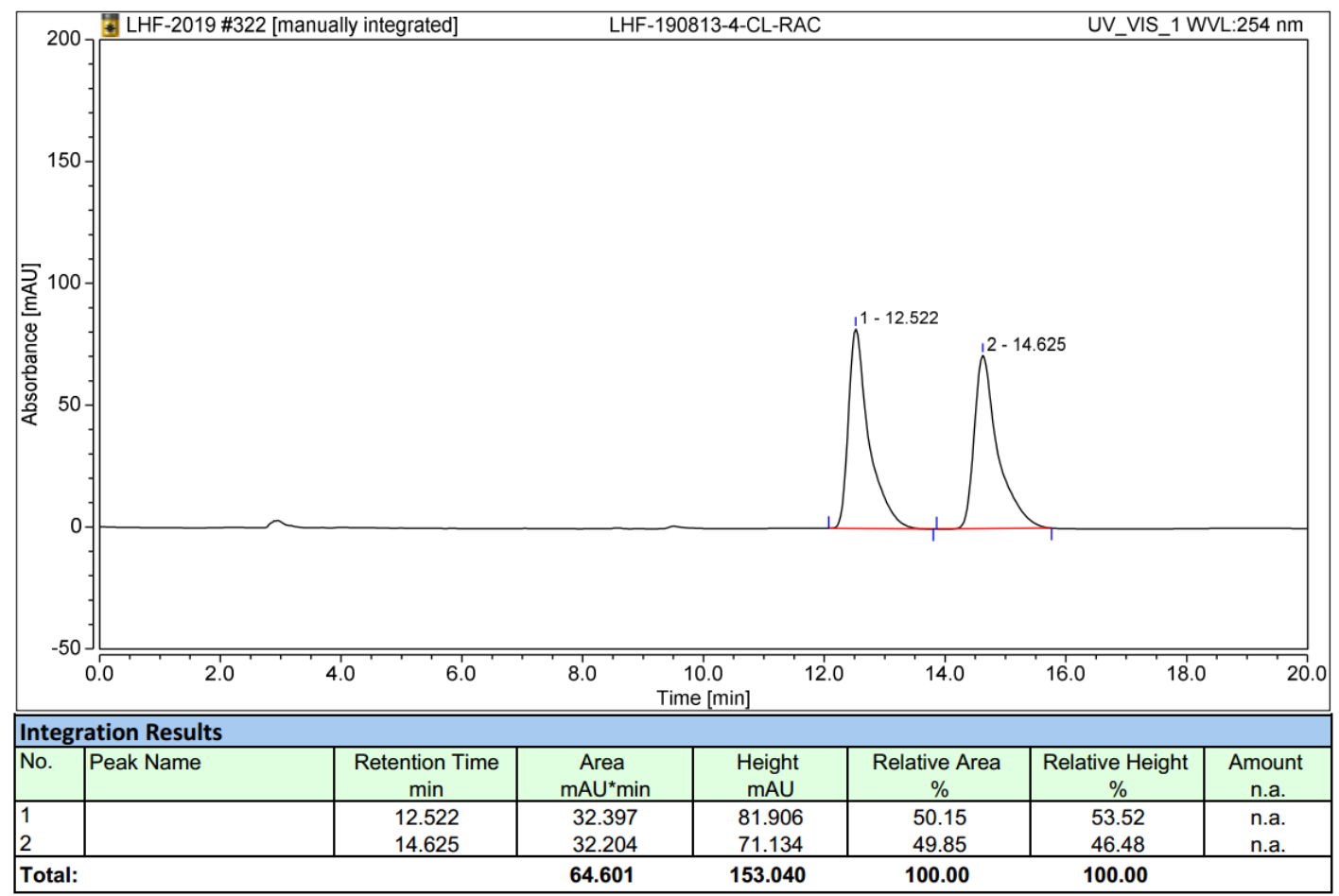




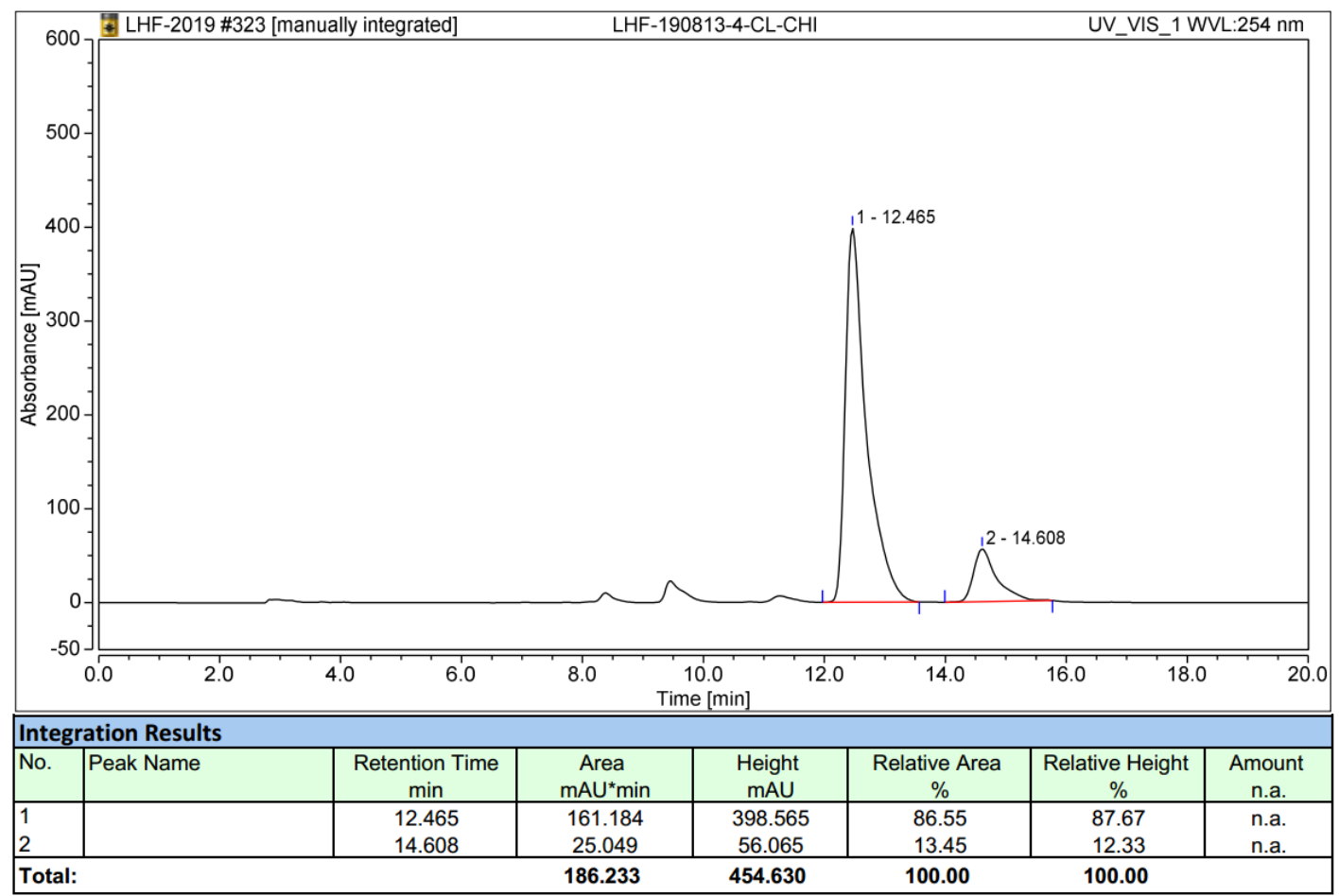

\begin{tabular}{|lll}
\hline Chromatogram & & \\
\hline
\end{tabular}

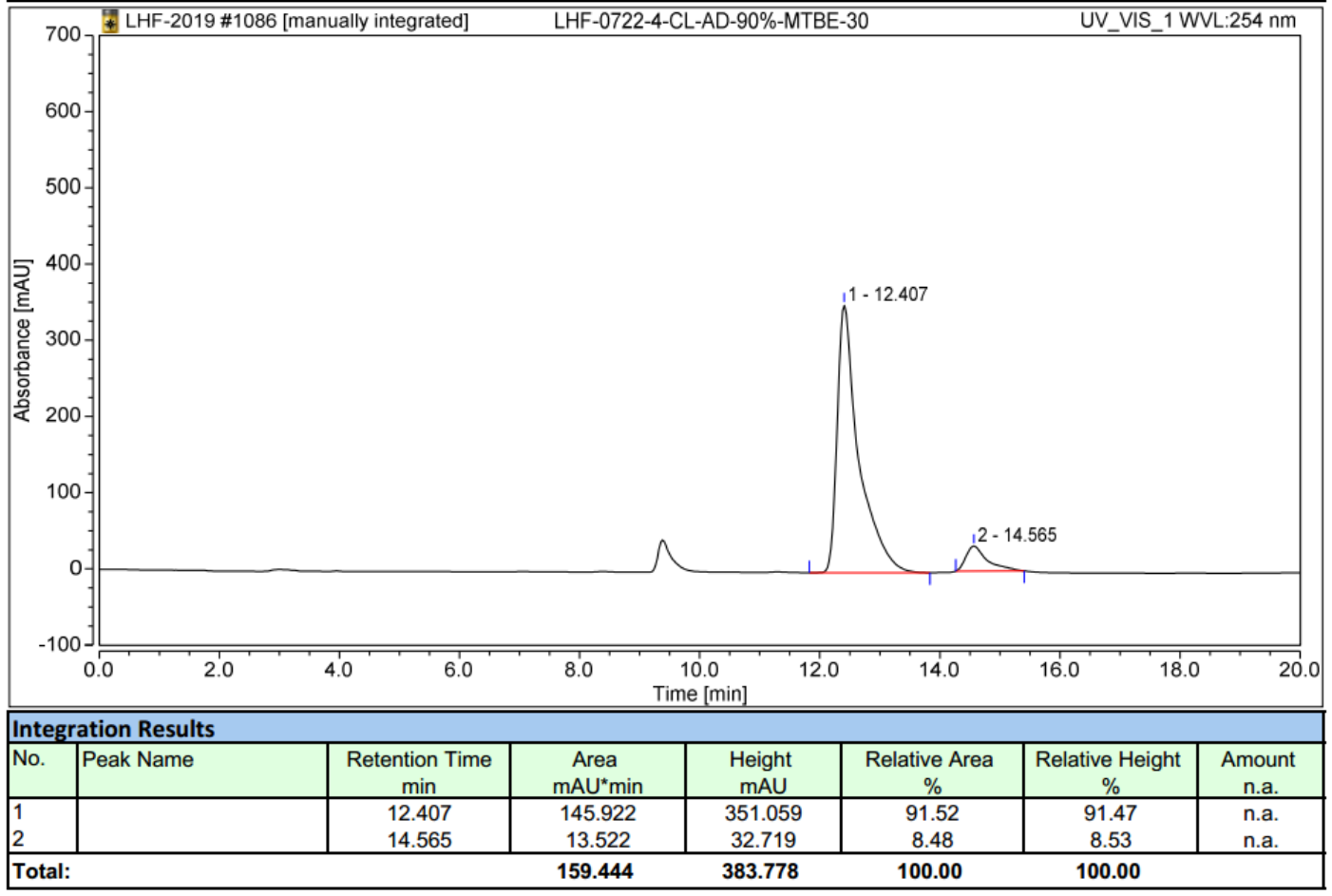<smiles>C=C(c1ccccc1)C(CO)n1cc([N+](=O)[O-])ccc1=O</smiles> 

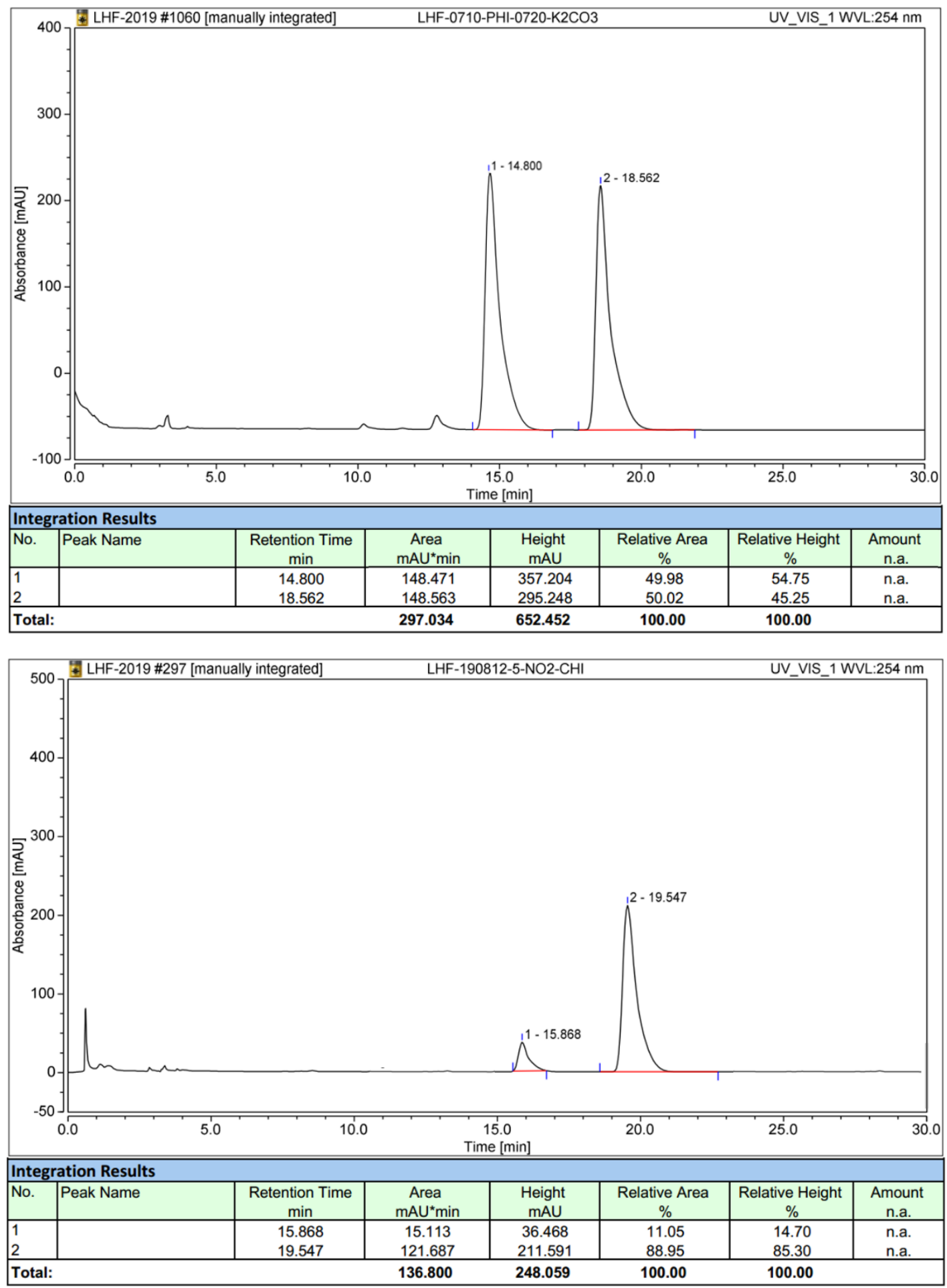


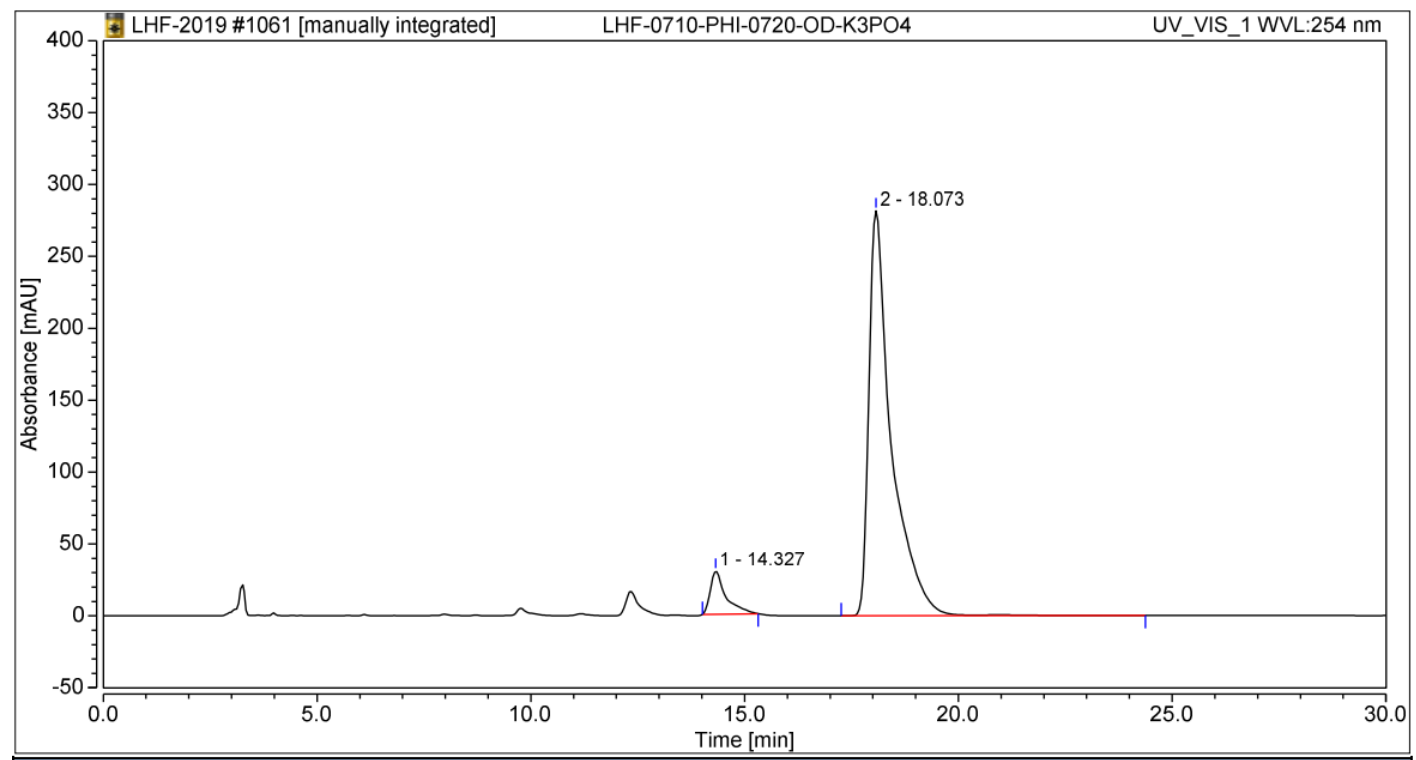

\begin{tabular}{|l|l|c|c|c|c|c|c|}
\hline \hline Integration Results \\
\hline No. & Peak Name & $\begin{array}{c}\text { Retention Time } \\
\text { min }\end{array}$ & $\begin{array}{c}\text { Area } \\
\mathrm{mAU} \text { min }\end{array}$ & $\begin{array}{c}\text { Height } \\
\mathrm{mAU}\end{array}$ & $\begin{array}{c}\text { Relative Area } \\
\%\end{array}$ & $\begin{array}{c}\text { Relative Height } \\
\%\end{array}$ & $\begin{array}{c}\text { Amount } \\
\text { n.a. }\end{array}$ \\
\hline 1 & & 14.327 & 12.716 & 29.931 & 6.90 & 9.61 & n.a. \\
2 & 18.073 & 171.445 & 281.643 & 93.10 & 90.39 & n.a. \\
\hline Total: & $\mathbf{1 8 4 . 1 6 1}$ & $\mathbf{3 1 1 . 5 7 4}$ & $\mathbf{1 0 0 . 0 0}$ & $\mathbf{1 0 0 . 0 0}$ \\
\hline
\end{tabular}<smiles>C=C(c1ccccc1)[C@H](CO)n1cc(C=O)ccc1=O</smiles>

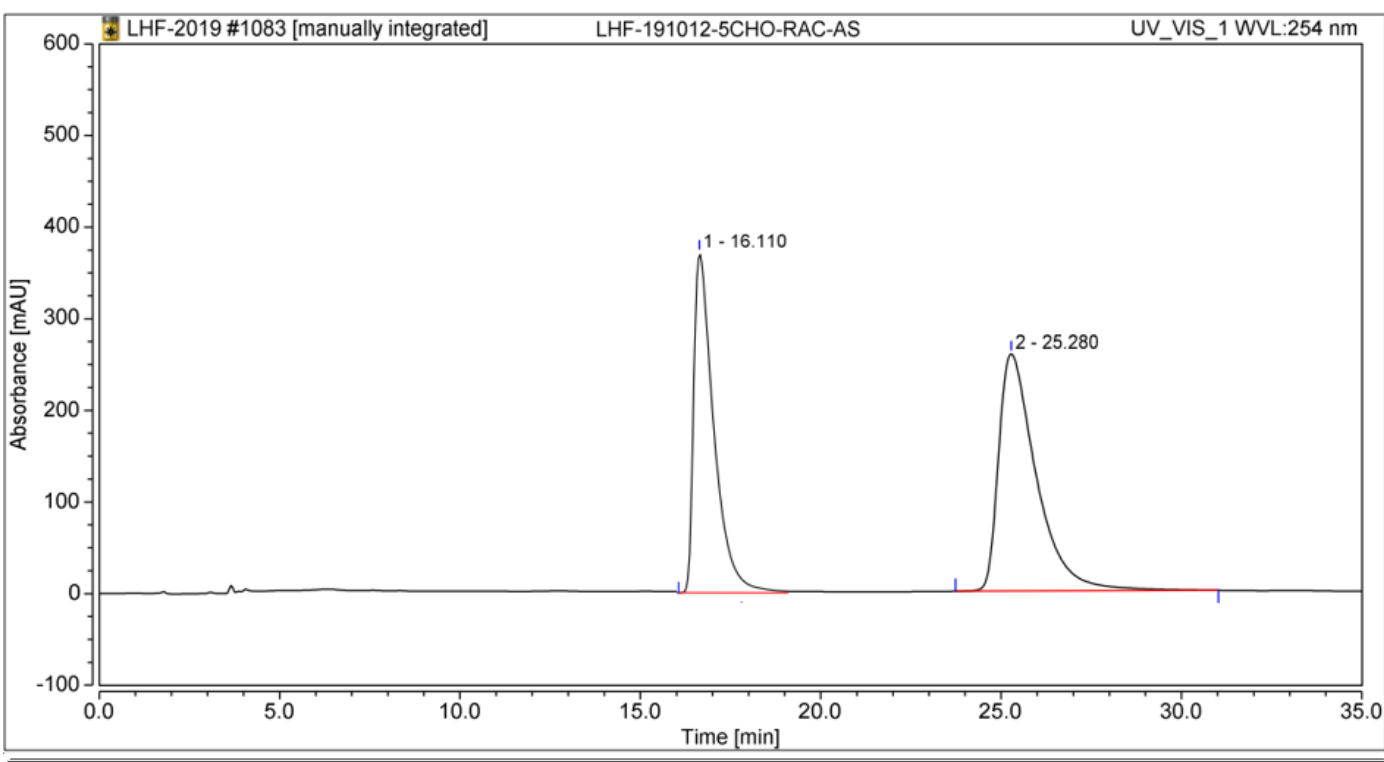

\begin{tabular}{|c|c|c|c|c|c|c|c|}
\hline \multicolumn{8}{|c|}{ Integration Results } \\
\hline No. & Peak Name & $\begin{array}{c}\text { Retention Time } \\
\text { min }\end{array}$ & $\begin{array}{c}\text { Area } \\
m A U^{*} \text { min }\end{array}$ & $\begin{array}{l}\text { Height } \\
\text { mAU }\end{array}$ & $\begin{array}{c}\text { Relative Area } \\
\%\end{array}$ & $\begin{array}{c}\text { Relative Height } \\
\%\end{array}$ & $\begin{array}{c}\text { Amount } \\
\text { n.a. }\end{array}$ \\
\hline $\begin{array}{l}1 \\
2\end{array}$ & & $\begin{array}{l}16.110 \\
25.280\end{array}$ & $\begin{array}{l}780.938 \\
781.014\end{array}$ & $\begin{array}{c}1046.032 \\
663.816\end{array}$ & $\begin{array}{l}50.00 \\
50.00\end{array}$ & $\begin{array}{l}61.18 \\
38.82\end{array}$ & $\begin{array}{l}\text { n.a. } \\
\text { n.a. }\end{array}$ \\
\hline \multicolumn{3}{|c|}{ Total: } & 1561.951 & 1709.848 & 100.00 & 100.00 & \\
\hline
\end{tabular}



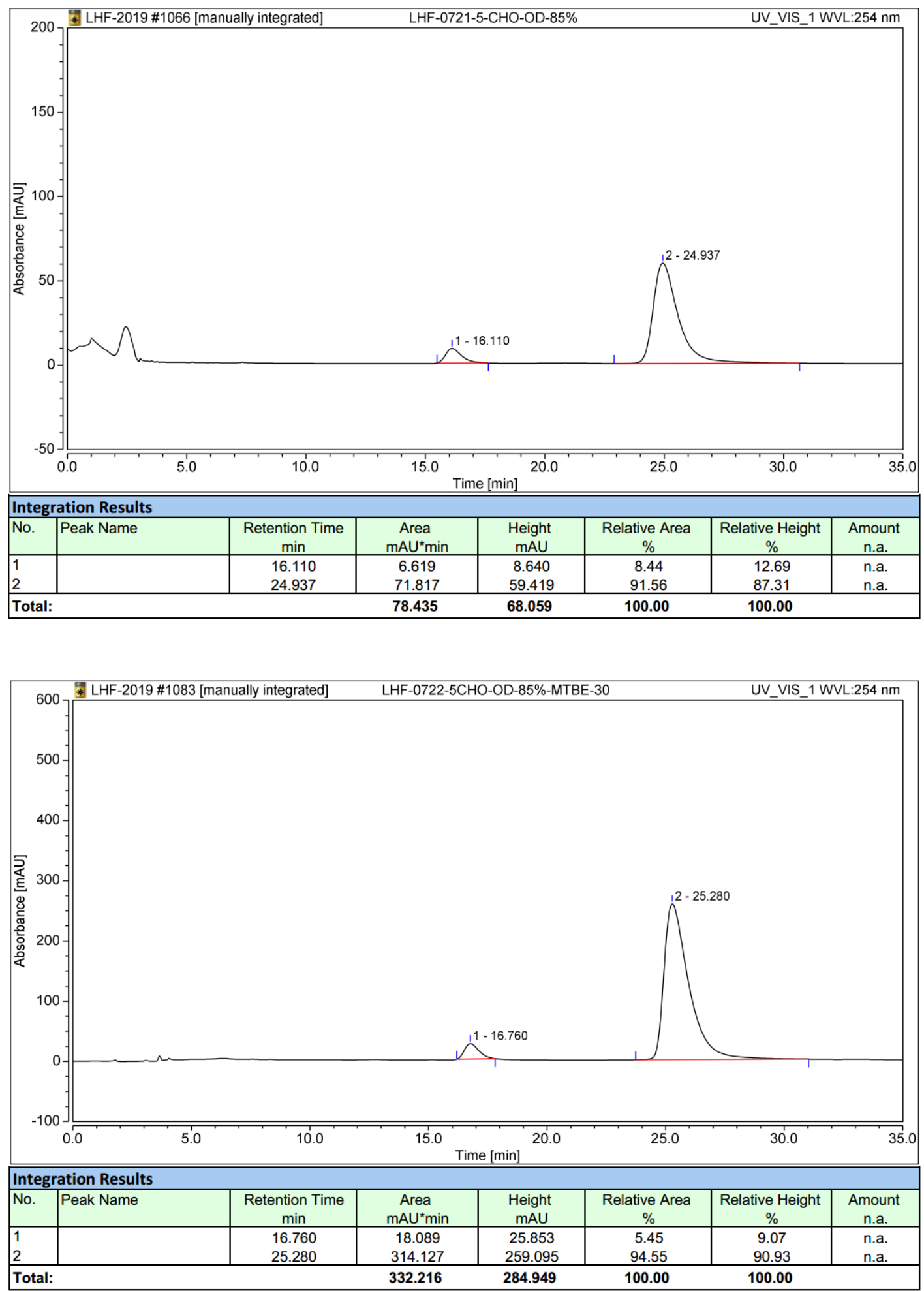<smiles></smiles> 

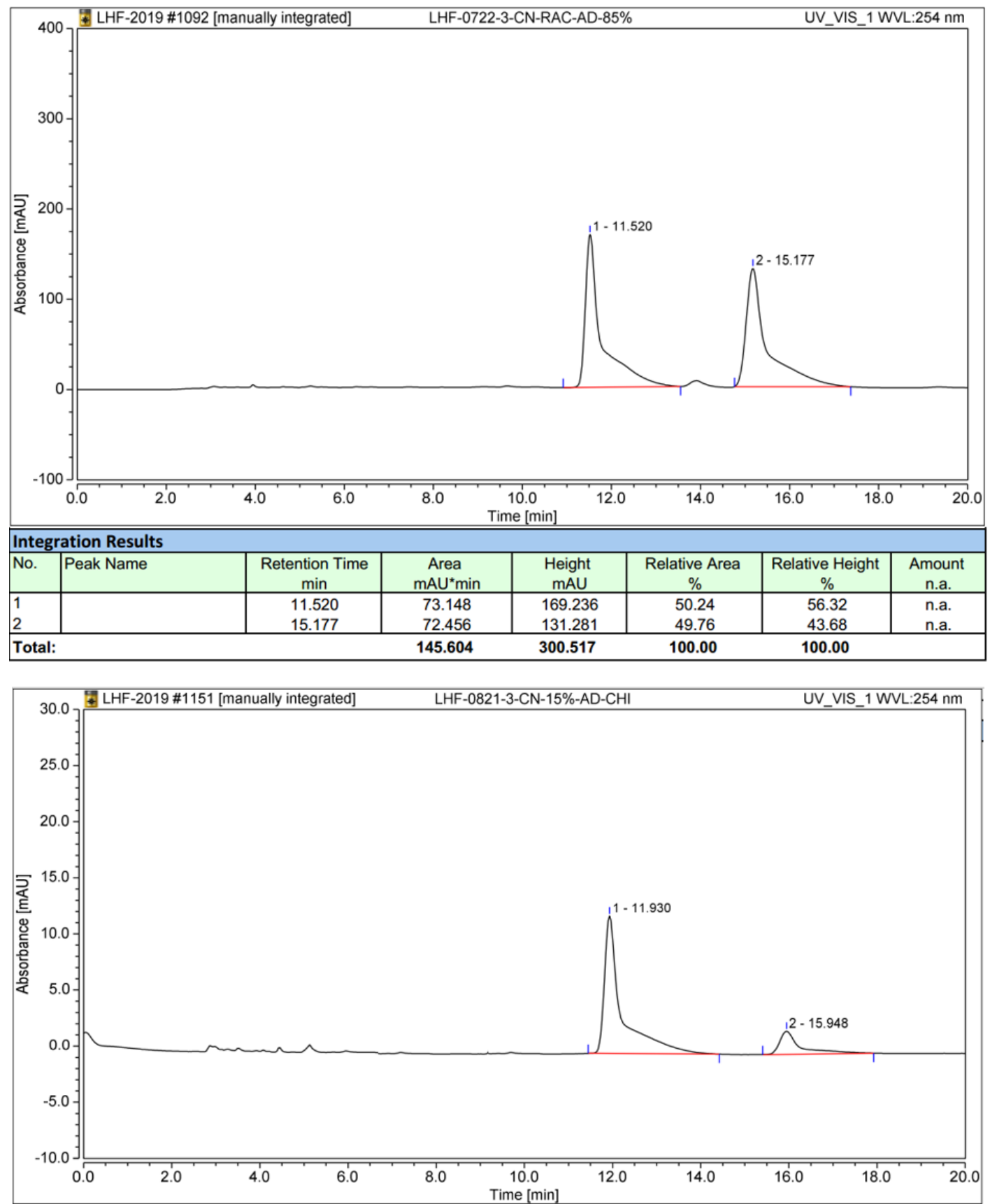

\begin{tabular}{|l|l|c|c|c|c|c|c}
\hline Integration Results \\
\hline No. & Peak Name & $\begin{array}{c}\text { Retention Time } \\
\text { min }\end{array}$ & $\begin{array}{c}\text { Area } \\
\text { mAU*min }\end{array}$ & $\begin{array}{c}\text { Height } \\
\text { mAU }\end{array}$ & $\begin{array}{c}\text { Relative Area } \\
\%\end{array}$ & $\begin{array}{c}\text { Relative Height } \\
\%\end{array}$ & $\begin{array}{c}\text { Amount } \\
\text { n.a. }\end{array}$ \\
\hline 1 & 11.930 & 5.604 & 12.242 & 85.23 & 85.63 & n.a. \\
2 & 15.948 & 1.129 & 2.055 & 14.77 & 14.37 & n.a. \\
\hline Total: & 6.733 & 14.298 & 100.00 & 100.00 \\
\hline
\end{tabular}




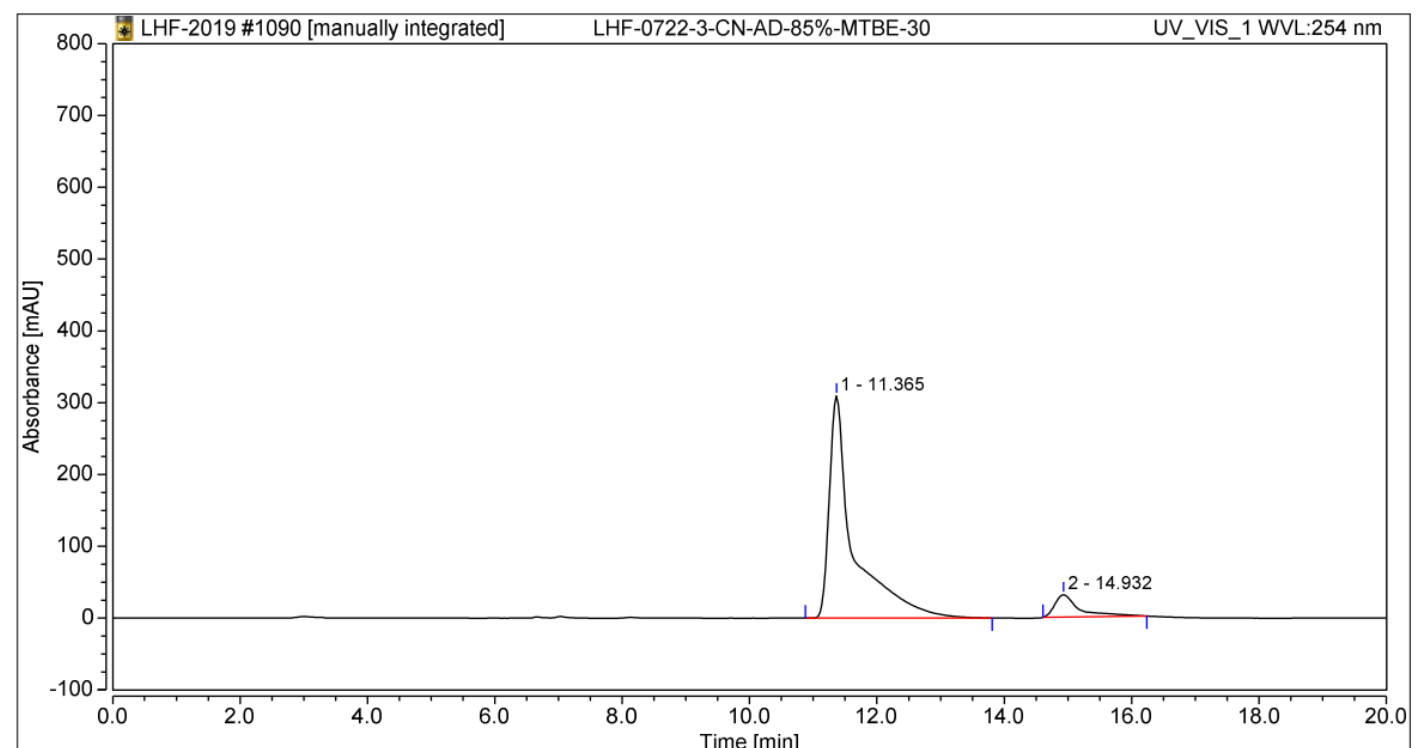

\begin{tabular}{|c|c|c|c|c|c|c|c|}
\hline \multicolumn{8}{|c|}{ Integration Results } \\
\hline No. & Peak Name & $\begin{array}{l}\text { Retention Time } \\
\text { min }\end{array}$ & $\begin{array}{c}\text { Area } \\
\mathrm{mAU}^{*} \min \end{array}$ & $\begin{array}{c}\text { Height } \\
\text { mAU }\end{array}$ & $\begin{array}{c}\text { Relative Area } \\
\%\end{array}$ & $\begin{array}{c}\text { Relative Height } \\
\%\end{array}$ & $\begin{array}{c}\text { Amount } \\
\text { n.a. }\end{array}$ \\
\hline $\begin{array}{l}1 \\
2\end{array}$ & & $\begin{array}{l}11.365 \\
14.932\end{array}$ & $\begin{array}{c}135.934 \\
13.927\end{array}$ & $\begin{array}{c}309.191 \\
31.110\end{array}$ & $\begin{array}{c}90.71 \\
9.29\end{array}$ & $\begin{array}{c}90.86 \\
9.14\end{array}$ & $\begin{array}{l}\text { n.a. } \\
\text { n.a. }\end{array}$ \\
\hline \multicolumn{3}{|c|}{ Total: } & 149.861 & 340.301 & 100.00 & 100.00 & \\
\hline
\end{tabular}<smiles>C=C(c1ccccc1)[C@H](CO)n1cc(Br)cc(Br)c1=O</smiles>

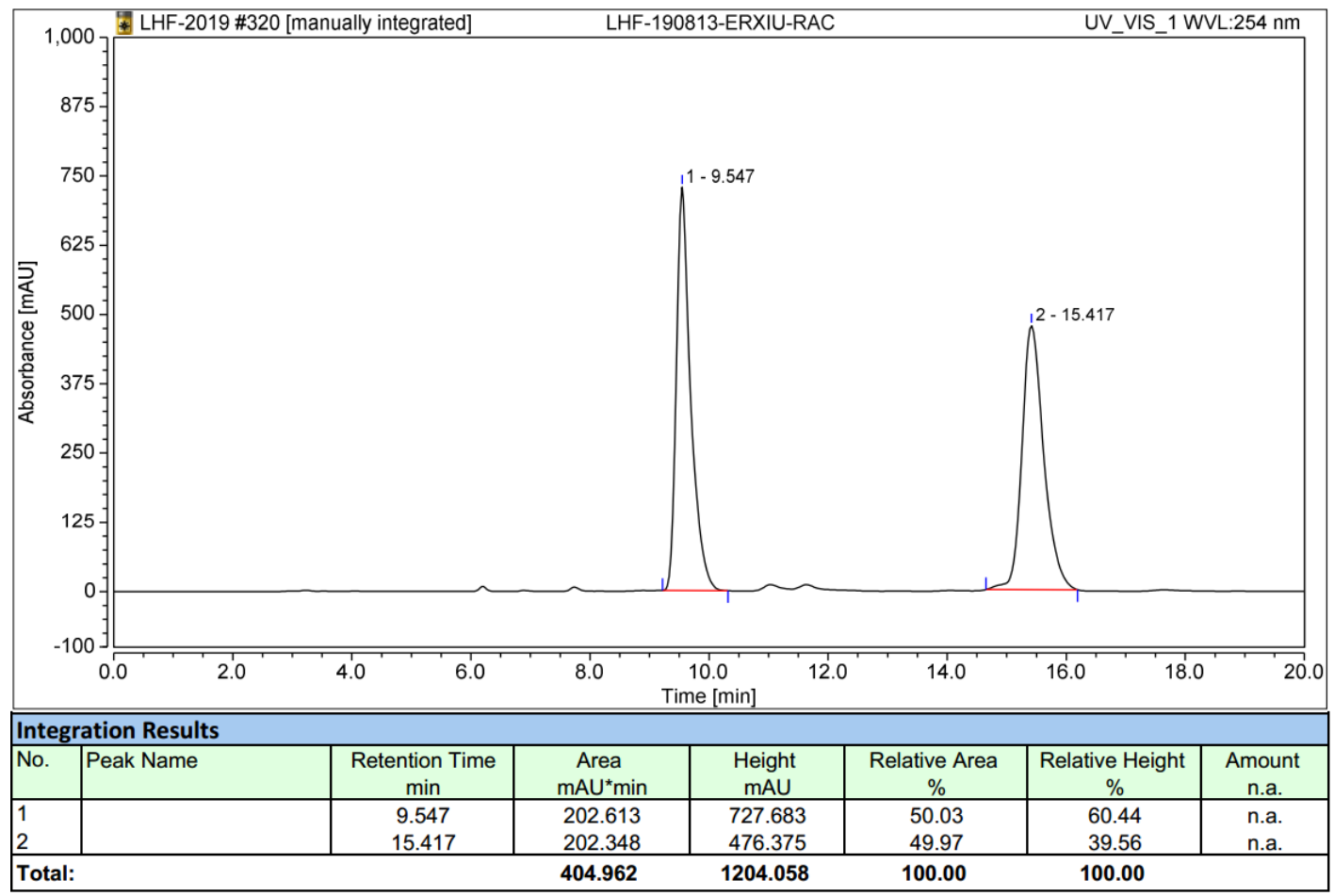



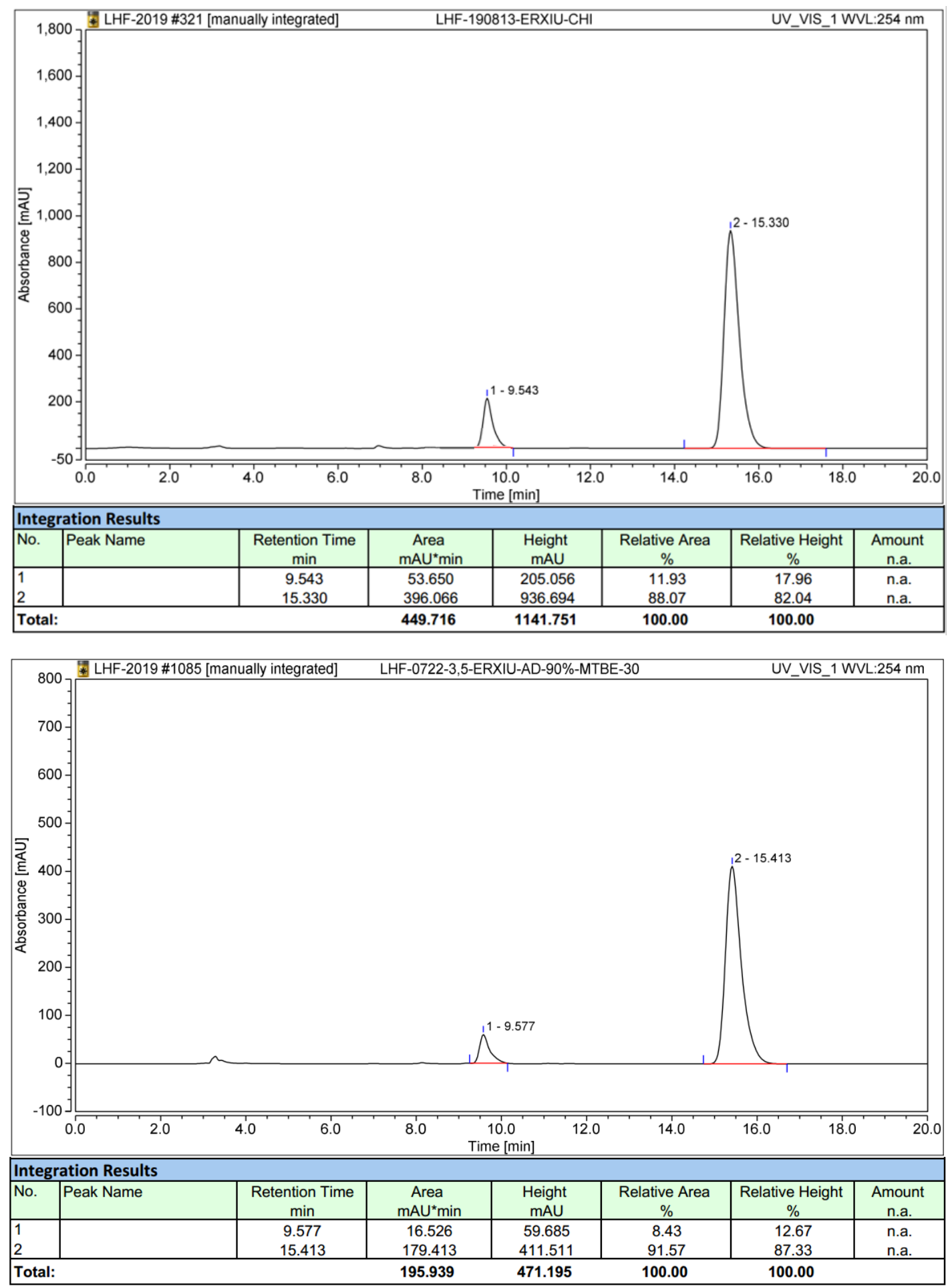<smiles></smiles> 

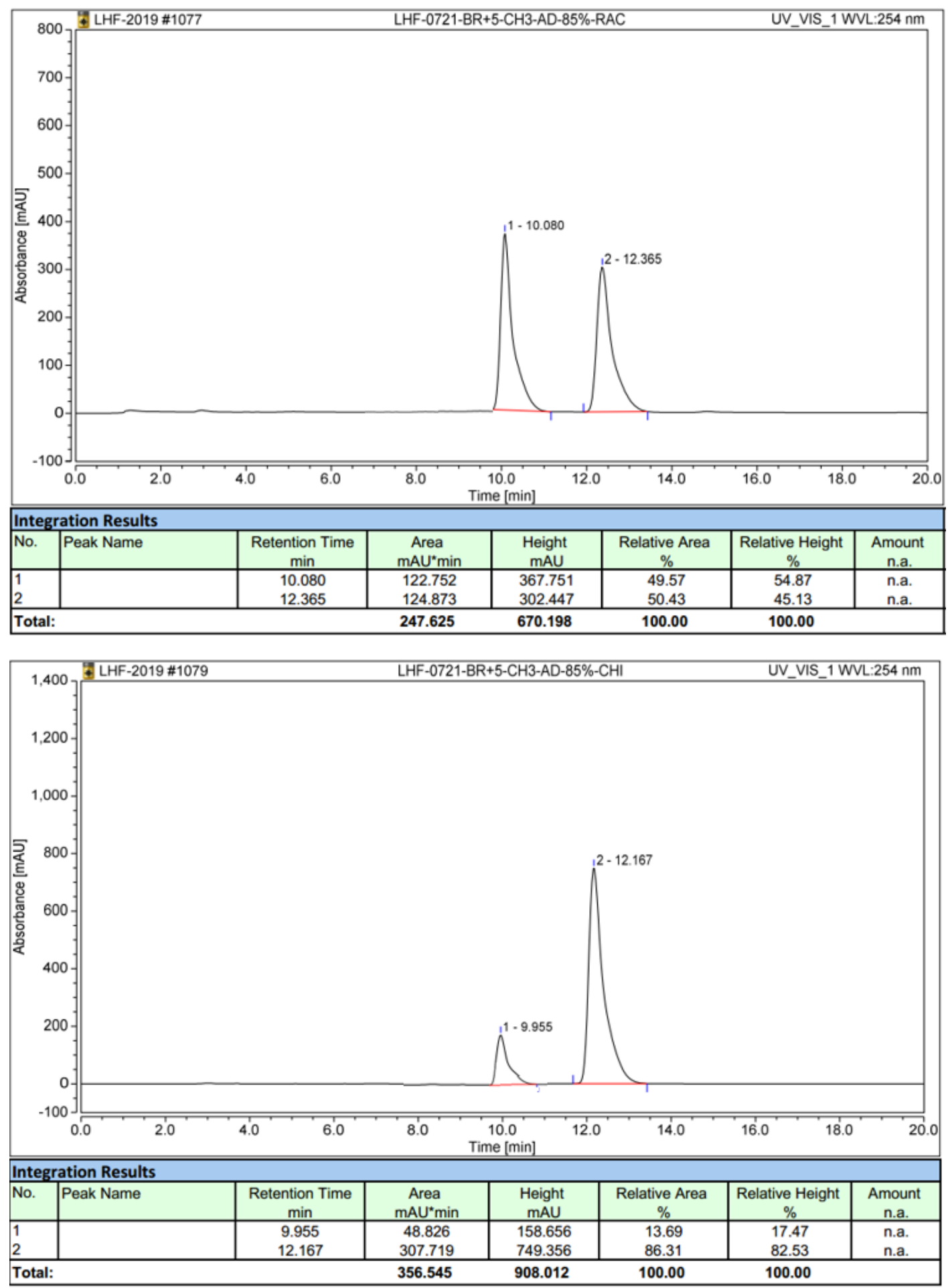


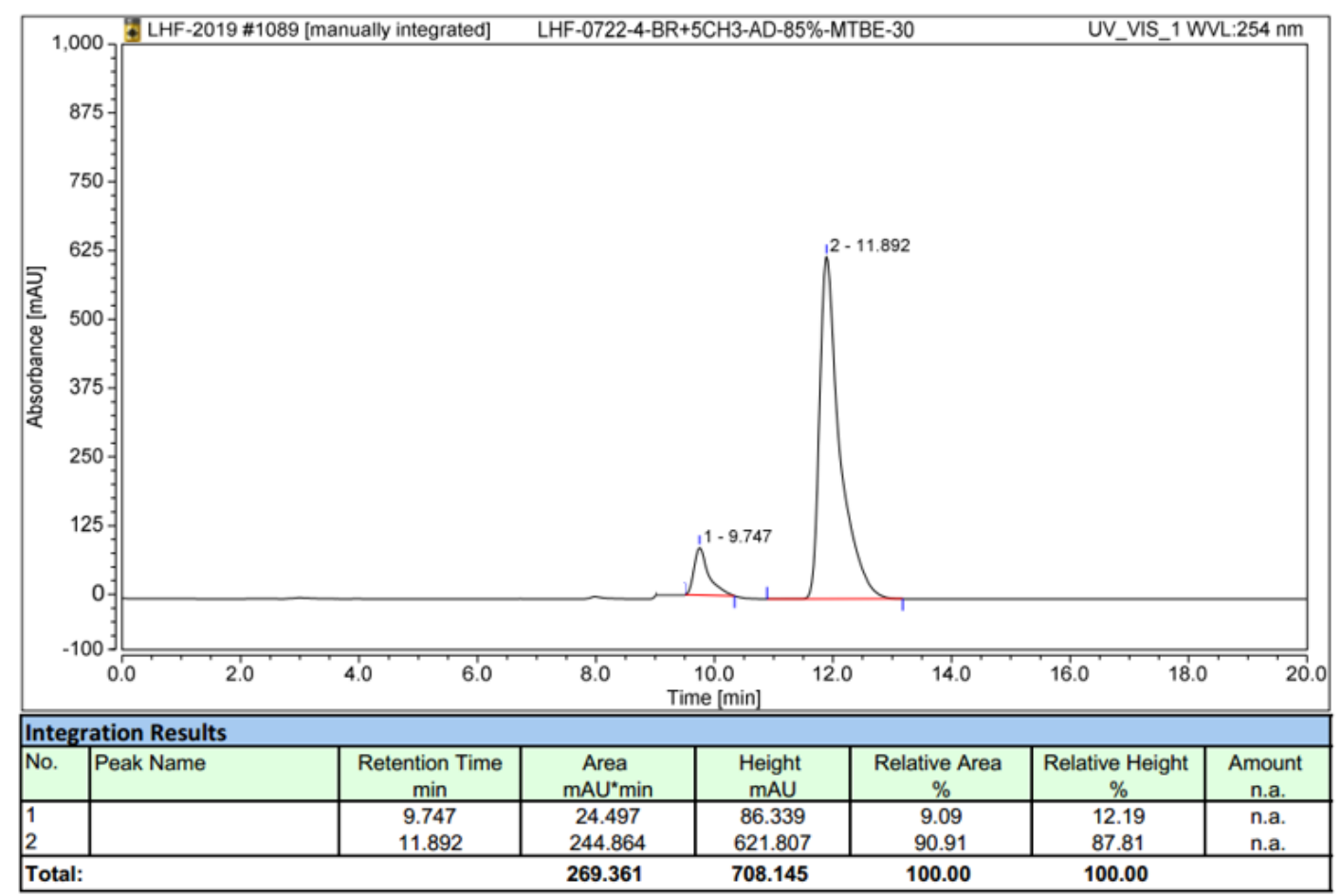<smiles></smiles>

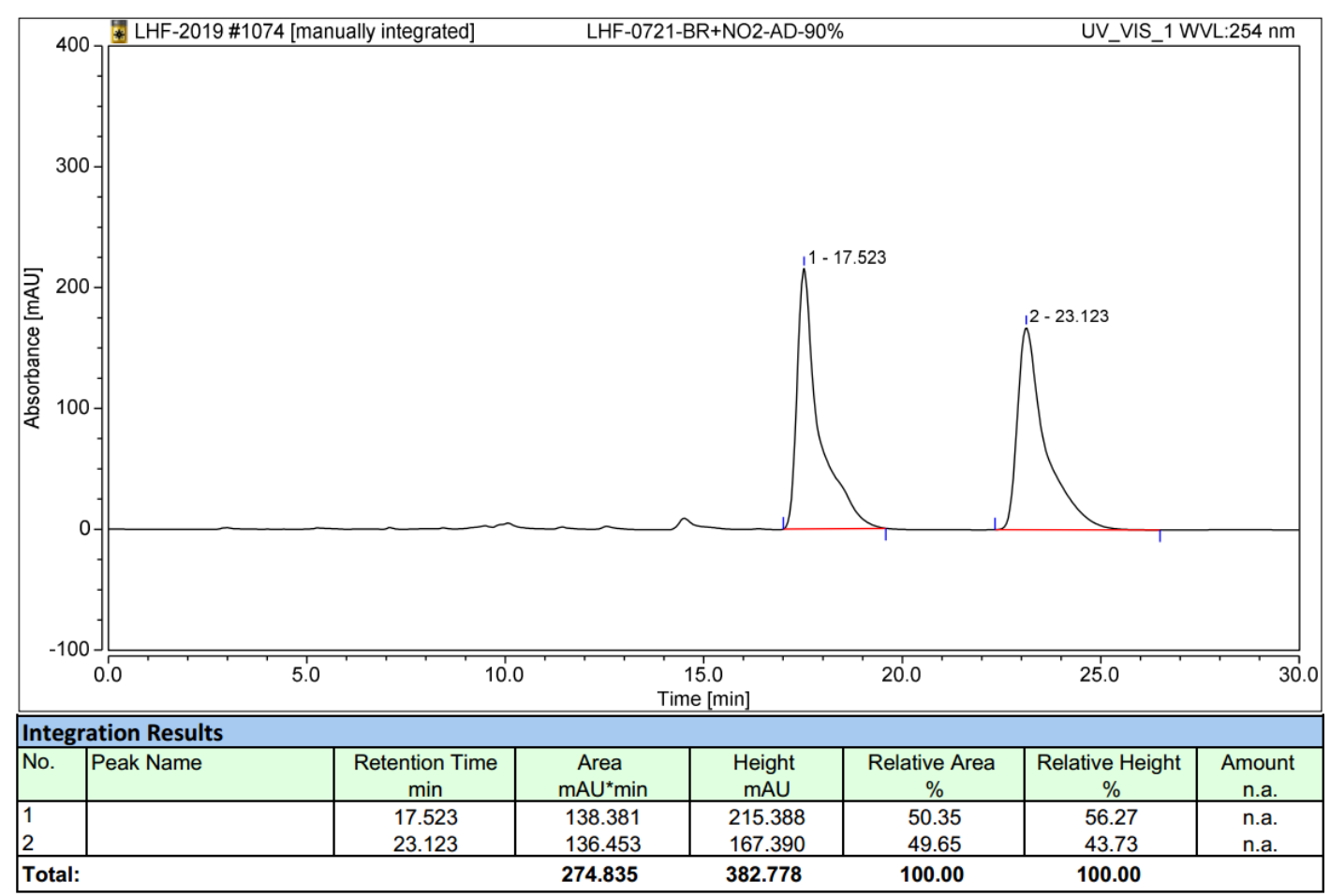



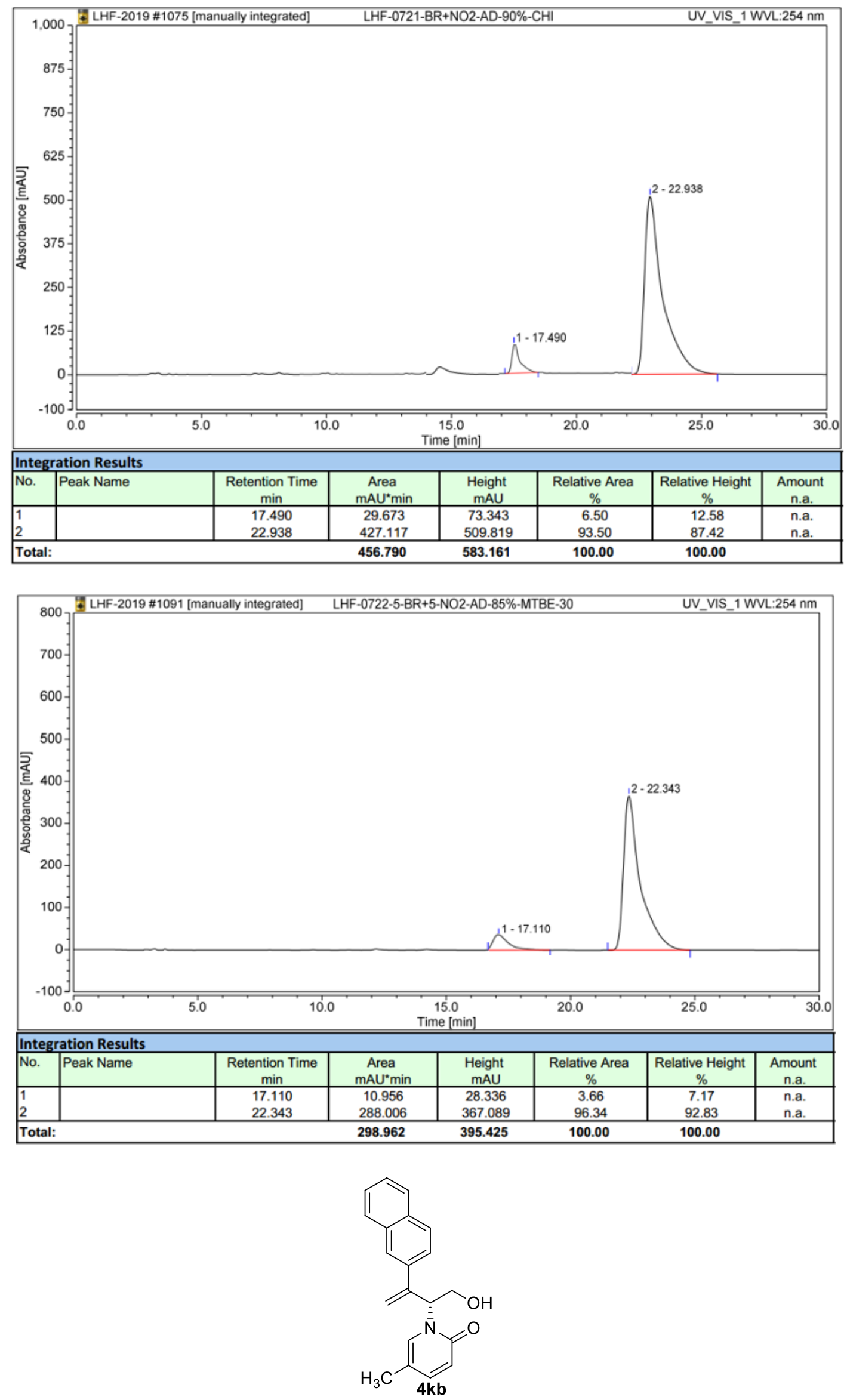


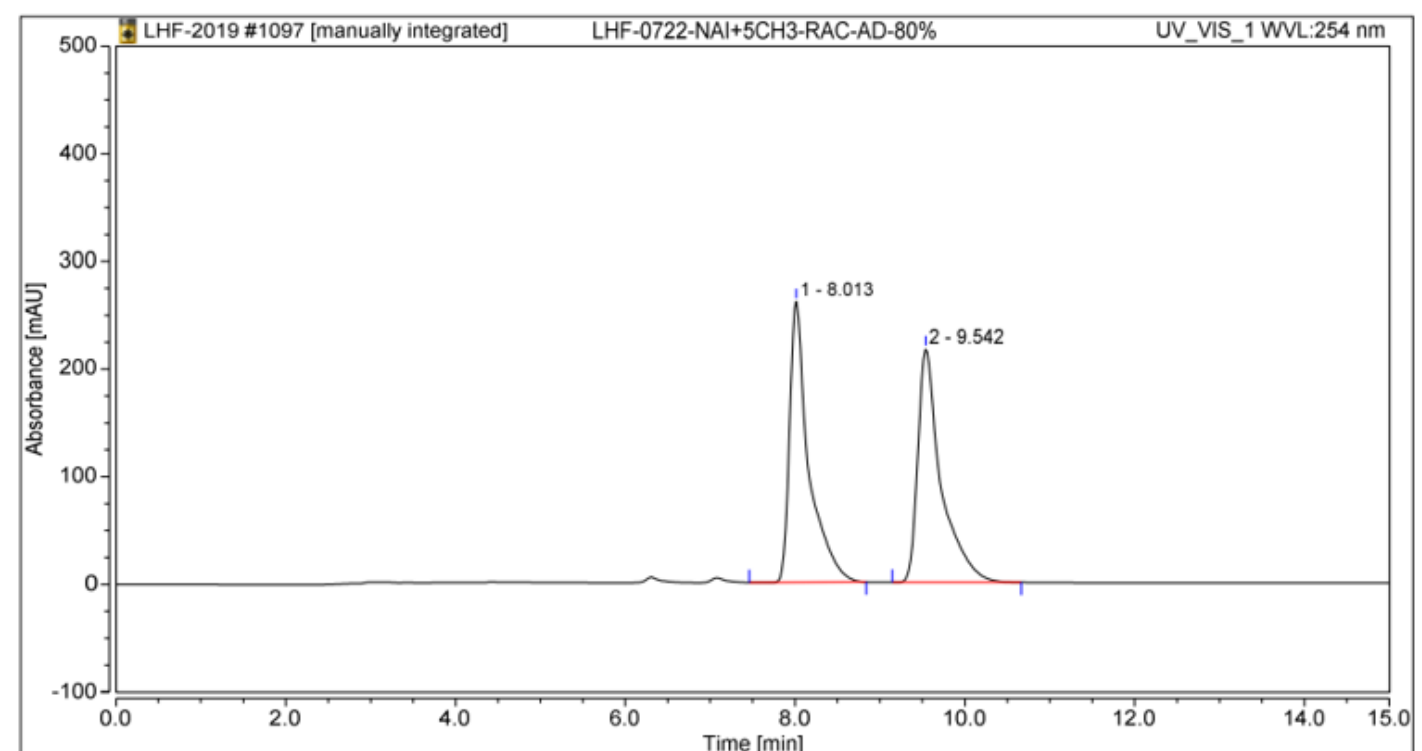

\begin{tabular}{|c|c|c|c|c|c|c|c|}
\hline \multicolumn{8}{|c|}{ Integration Results } \\
\hline No. & Peak Name & $\begin{array}{c}\text { Retention Time } \\
\text { min }\end{array}$ & $\begin{array}{c}\text { Area } \\
\text { mAU*min }\end{array}$ & $\begin{array}{l}\text { Height } \\
\text { mAU }\end{array}$ & $\begin{array}{c}\text { Relative Area } \\
\% \\
\end{array}$ & $\begin{array}{c}\text { Relative Height } \\
\% \\
\end{array}$ & $\begin{array}{c}\text { Amount } \\
\text { n.a. }\end{array}$ \\
\hline $\begin{array}{l}1 \\
2 \\
\end{array}$ & & $\begin{array}{r}8.013 \\
9.542 \\
\end{array}$ & $\begin{array}{l}68.042 \\
68.488 \\
\end{array}$ & $\begin{array}{l}261.024 \\
216.903 \\
\end{array}$ & $\begin{array}{r}49.84 \\
50.16 \\
\end{array}$ & $\begin{array}{r}54.62 \\
45.38 \\
\end{array}$ & $\begin{array}{l}\text { n.a. } \\
\text { n.a. }\end{array}$ \\
\hline \multicolumn{3}{|c|}{ Total: } & 136.529 & 477.927 & 100.00 & 100.00 & \\
\hline
\end{tabular}

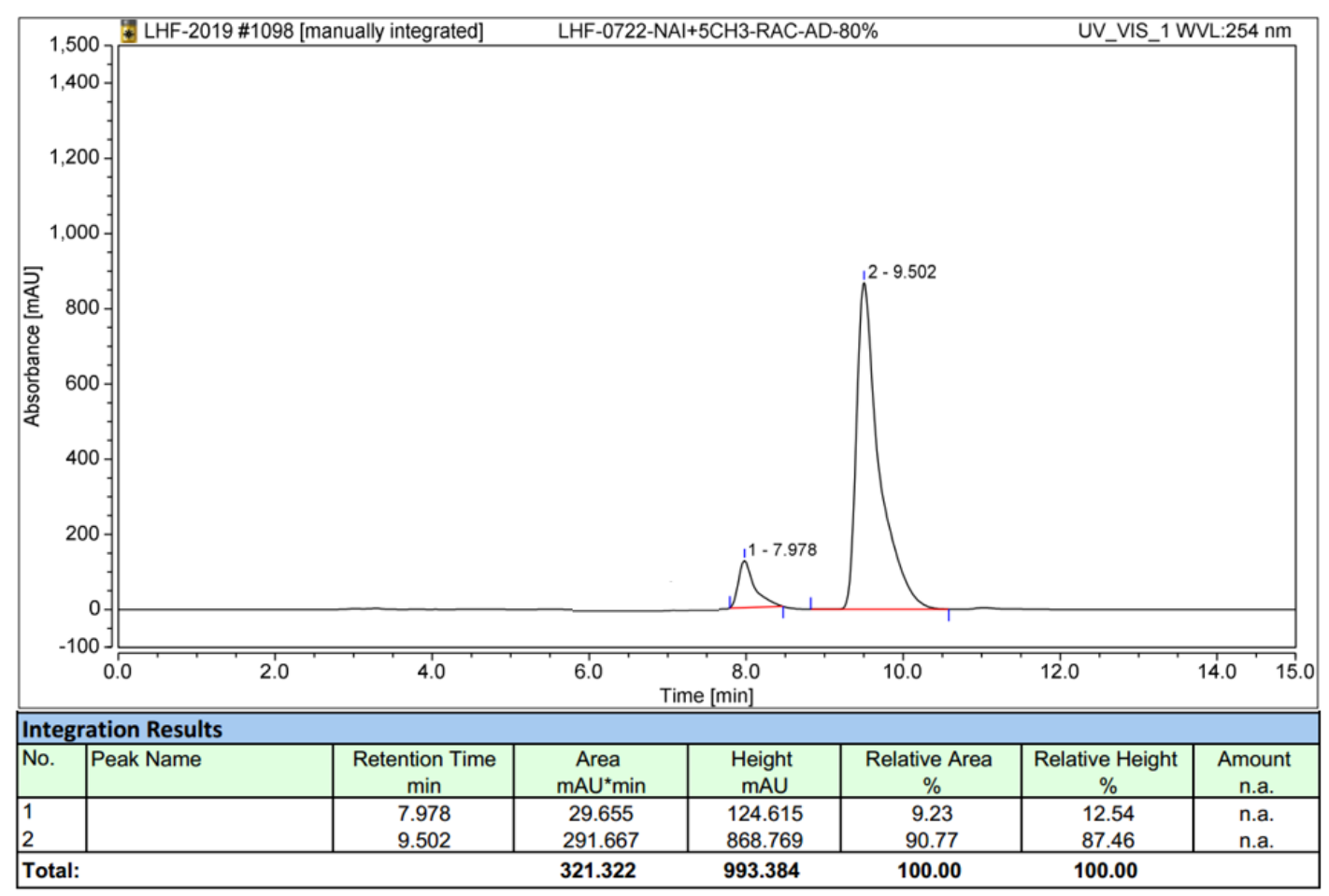



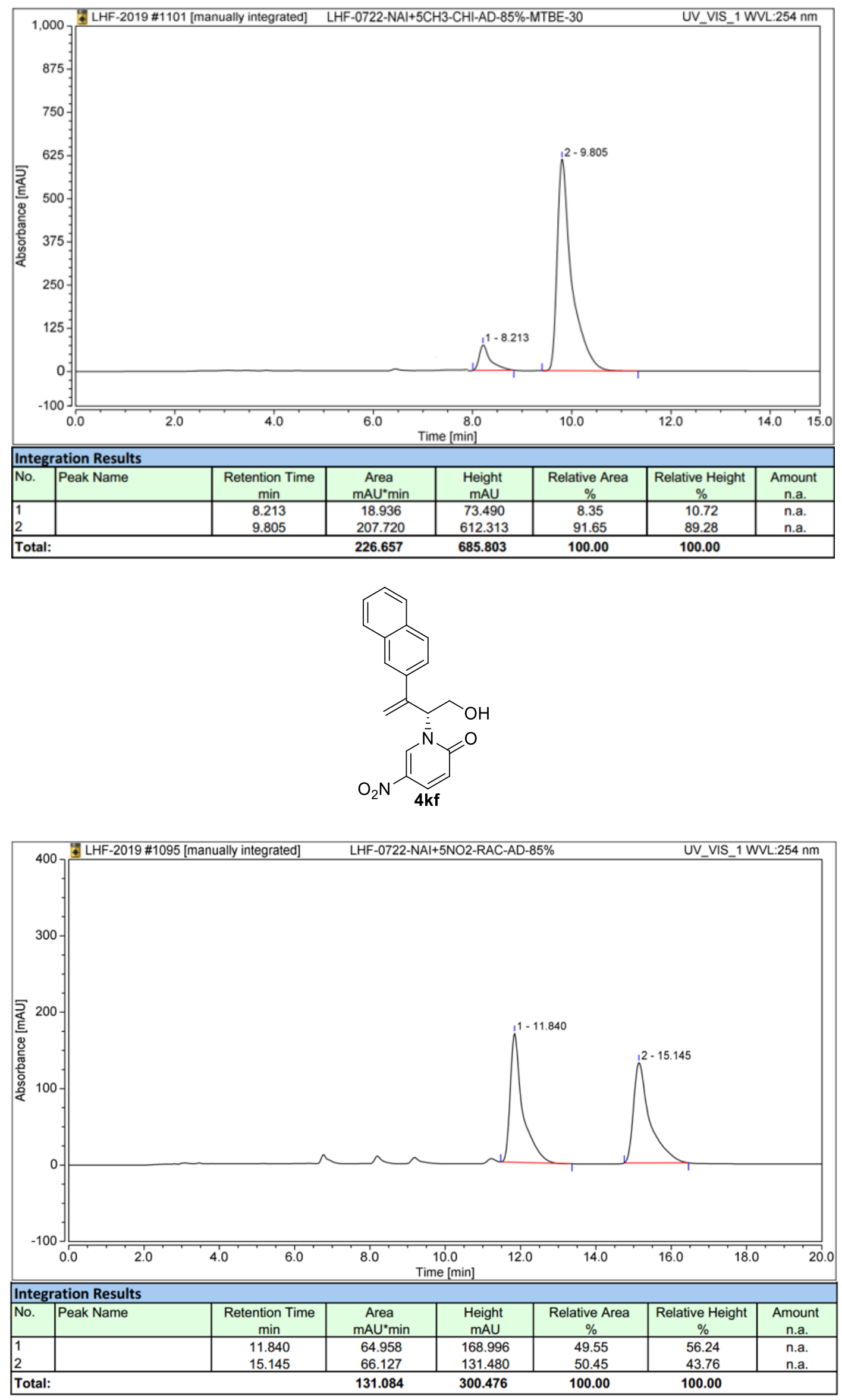

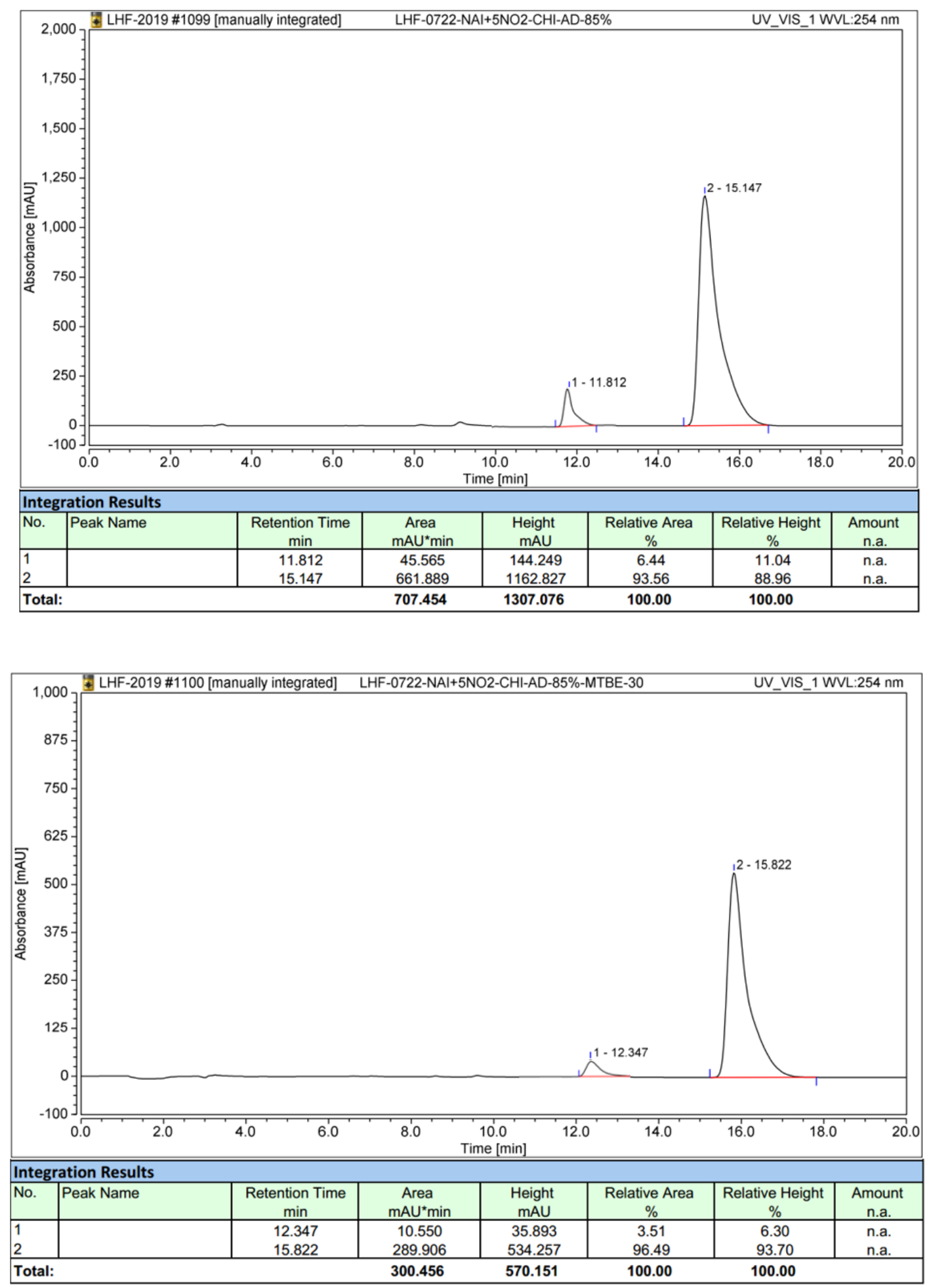
<smiles></smiles>
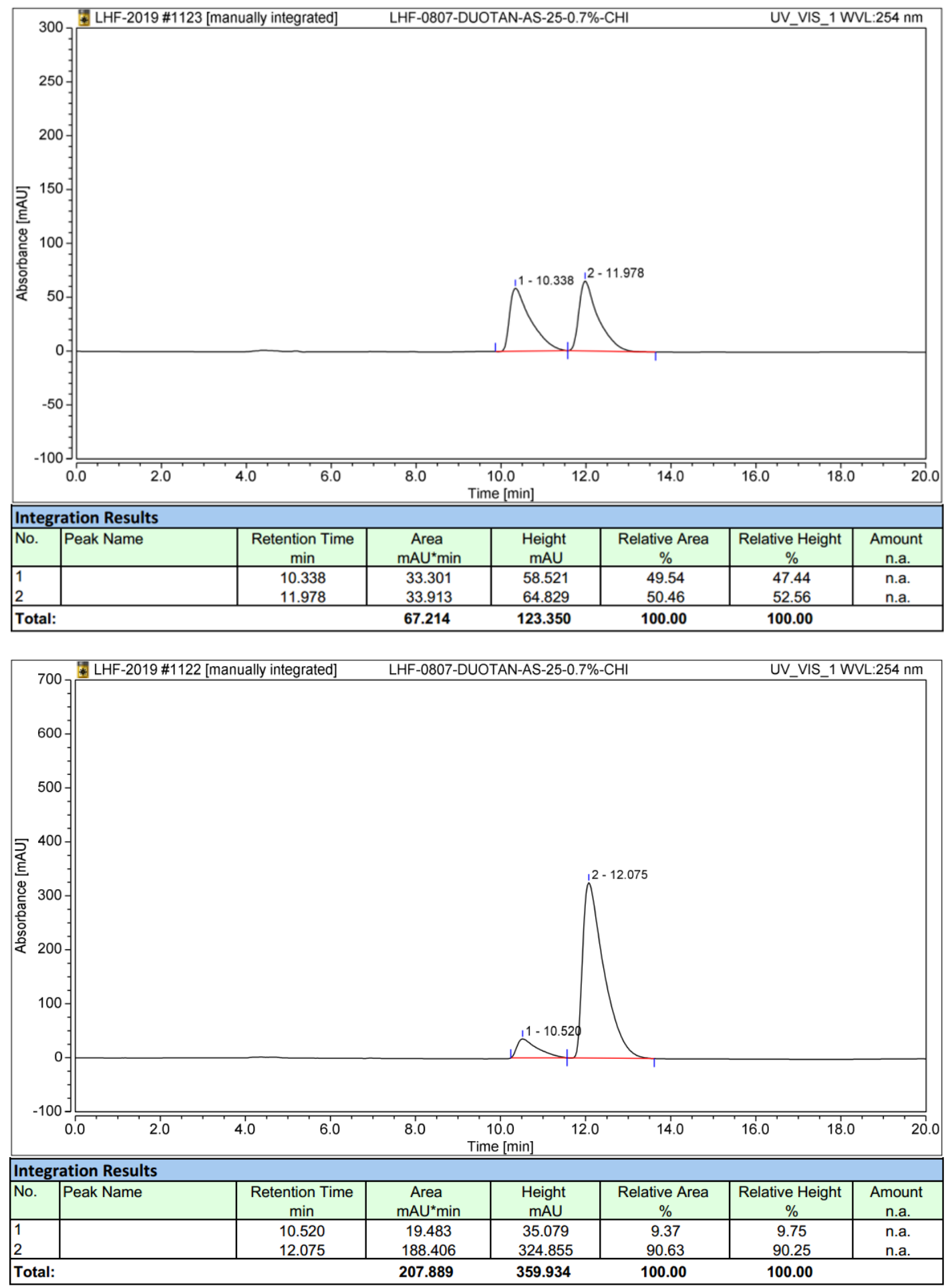
<smiles>C=C(c1ccccc1)[C@H](CCO)n1cc(C)[12cH]cc1=O</smiles>
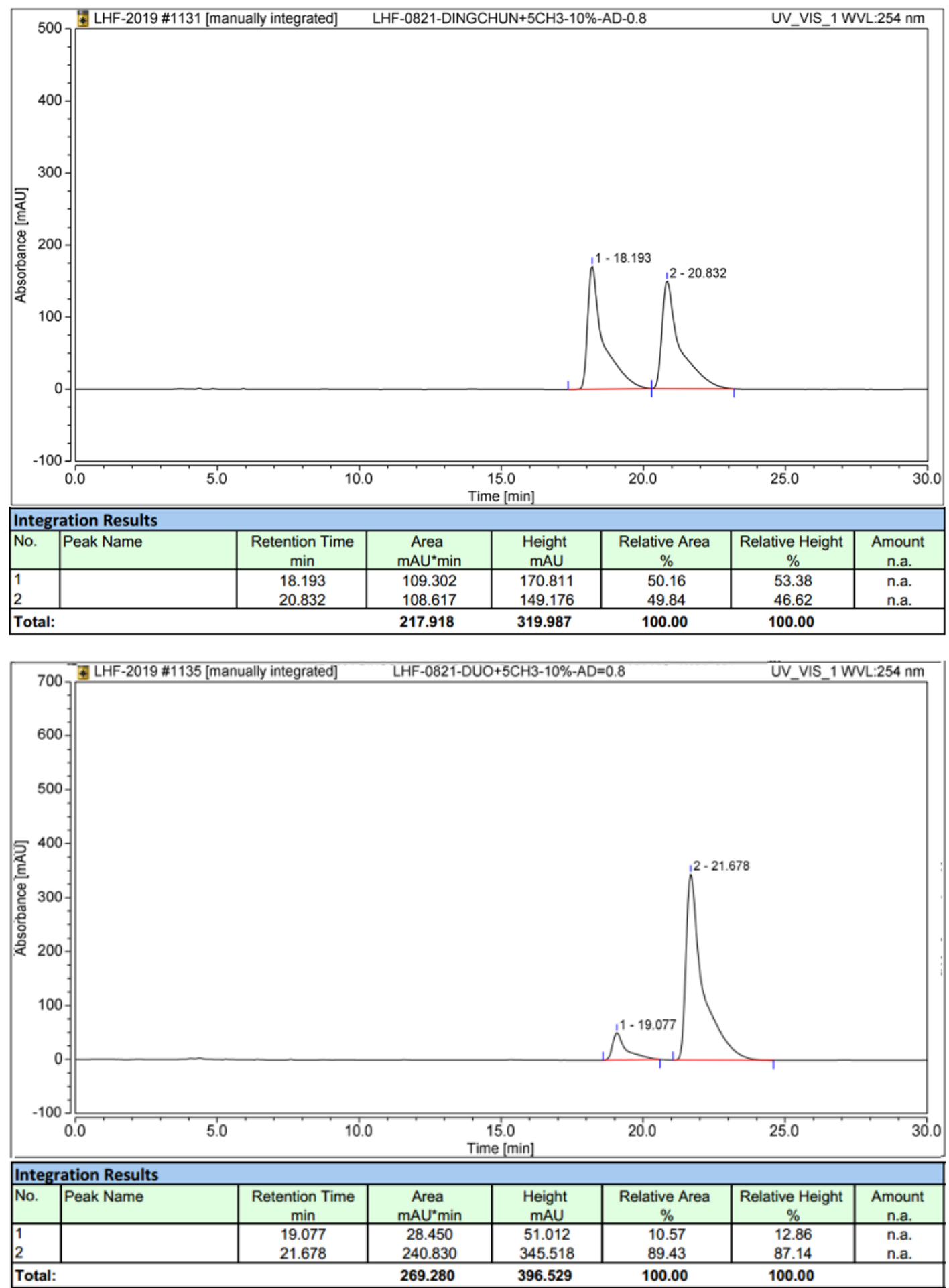
<smiles>C=C(c1ccccc1)[C@H](CCO)n1cc(C=O)ccc1=O</smiles>
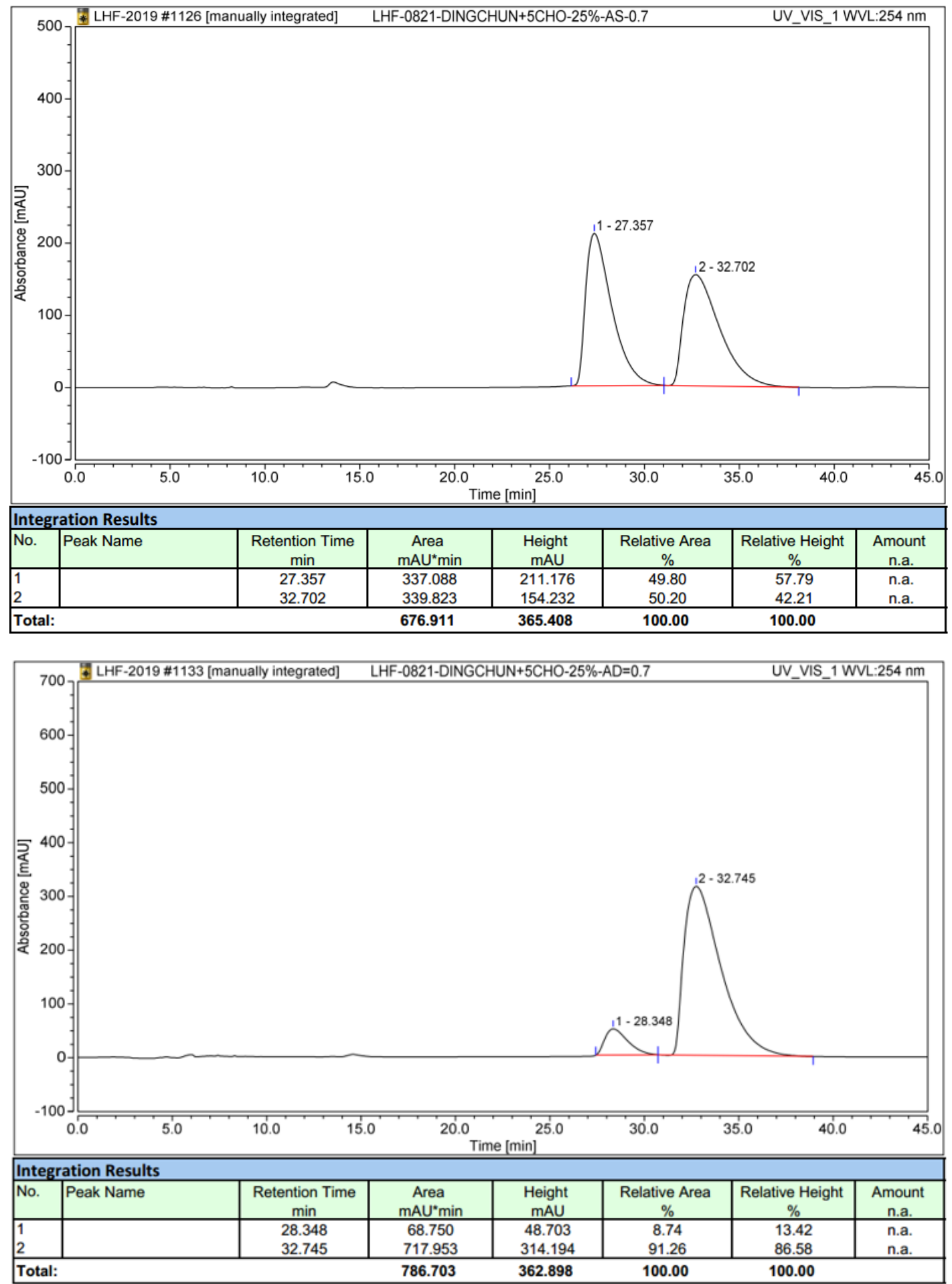

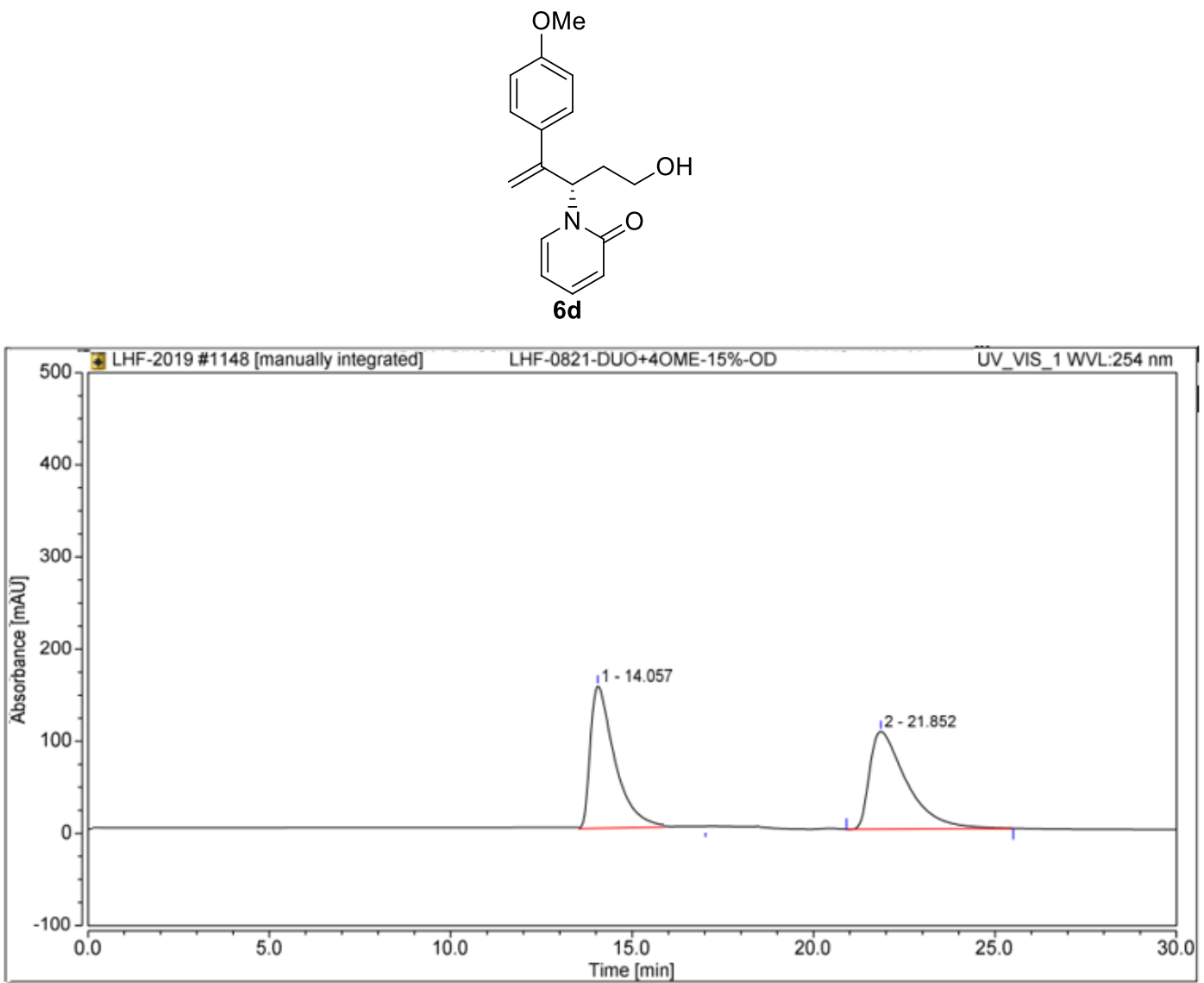

\begin{tabular}{|l|l|c|c|c|c|c|c|}
\hline Integration Results \\
\hline No. & Peak Name & $\begin{array}{c}\text { Retention Time } \\
\min \end{array}$ & $\begin{array}{c}\text { Area } \\
\text { mAU*min }\end{array}$ & $\begin{array}{c}\text { Height } \\
\text { mAU }\end{array}$ & $\begin{array}{c}\text { Relative Area } \\
\%\end{array}$ & $\begin{array}{c}\text { Relative Height } \\
\%\end{array}$ & $\begin{array}{c}\text { Amount } \\
\text { n.a. }\end{array}$ \\
\hline 1 & & 14.057 & 125.363 & 154.188 & 50.09 & 59.29 & n.a. \\
2 & 21.852 & 124.932 & 105.861 & 49.91 & 40.71 & n.a. \\
\hline
\end{tabular}

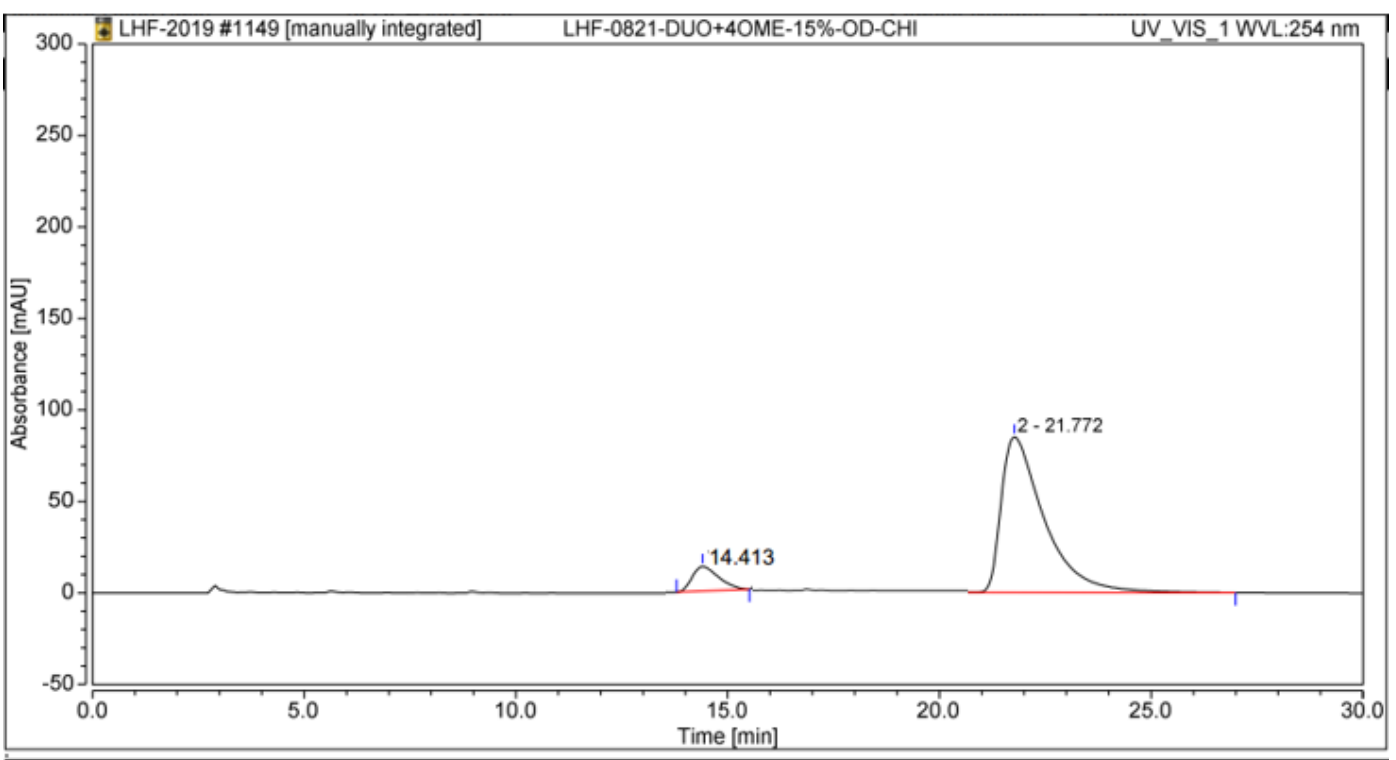

\begin{tabular}{|c|c|c|c|c|c|c|c|}
\hline \multicolumn{8}{|c|}{ Integration Results } \\
\hline No. & Peak Name & $\begin{array}{c}\text { Retention Time } \\
\text { min }\end{array}$ & $\begin{array}{c}\text { Area } \\
\text { mAU*min }\end{array}$ & $\begin{array}{c}\text { Height } \\
\text { mAU }\end{array}$ & $\begin{array}{c}\text { Relative Area } \\
\%\end{array}$ & $\begin{array}{c}\text { Relative Height } \\
\%\end{array}$ & $\begin{array}{c}\text { Amount } \\
\text { n.a. }\end{array}$ \\
\hline 1 & & 14.413 & 9.750 & 13.397 & 8.86 & 13.61 & n.a. \\
\hline 2 & & 21.772 & 100.327 & 85.041 & 91.14 & 86.39 & n.a. \\
\hline \multicolumn{3}{|c|}{ Total: } & 110.077 & 98.437 & 100.00 & 100.00 & \\
\hline
\end{tabular}


<smiles>C=C(c1ccc(Br)cc1)C(CCO)N1C=Cc2ccc(cc2)C1=O</smiles>

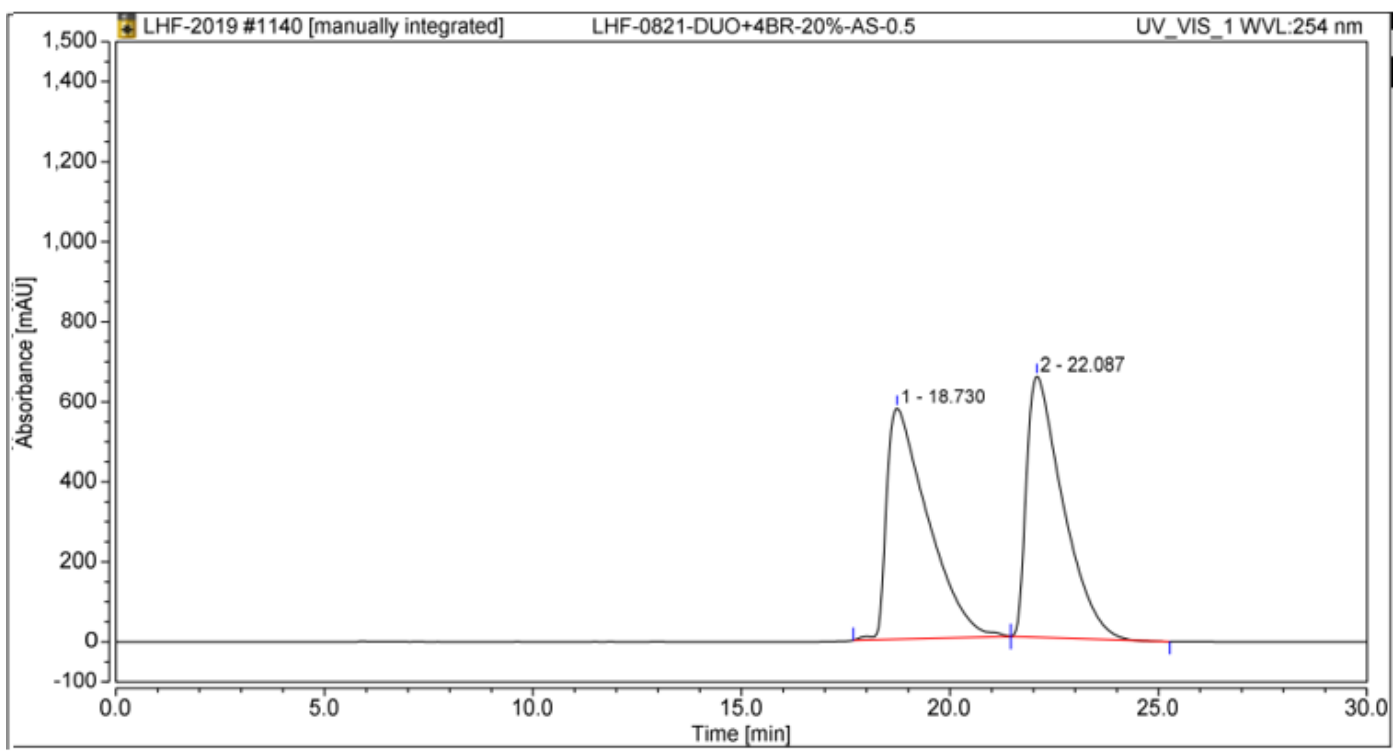

\begin{tabular}{|c|c|c|c|c|c|c|c|}
\hline \multicolumn{8}{|c|}{ Integration Results } \\
\hline No. & Peak Name & $\begin{array}{c}\text { Retention Time } \\
\text { min }\end{array}$ & $\begin{array}{c}\text { Area } \\
\mathrm{mAU} \text { min }\end{array}$ & $\begin{array}{c}\text { Height } \\
\text { mAU }\end{array}$ & $\begin{array}{c}\text { Relative Area } \\
\% \\
\end{array}$ & $\begin{array}{c}\text { Relative Height } \\
\%\end{array}$ & $\begin{array}{c}\text { Amount } \\
\text { n.a. }\end{array}$ \\
\hline $\begin{array}{l}1 \\
2\end{array}$ & & $\begin{array}{l}18.730 \\
22.087 \\
\end{array}$ & $\begin{array}{l}661.583 \\
661.872\end{array}$ & $\begin{array}{l}577.351 \\
652.081\end{array}$ & $\begin{array}{l}49.99 \\
50.01\end{array}$ & $\begin{array}{l}46.96 \\
53.04 \\
\end{array}$ & $\begin{array}{l}\text { n.a. } \\
\text { n.a. }\end{array}$ \\
\hline \multicolumn{3}{|c|}{ Total: } & 1323.455 & 1229.432 & 100.00 & 100.00 & \\
\hline
\end{tabular}

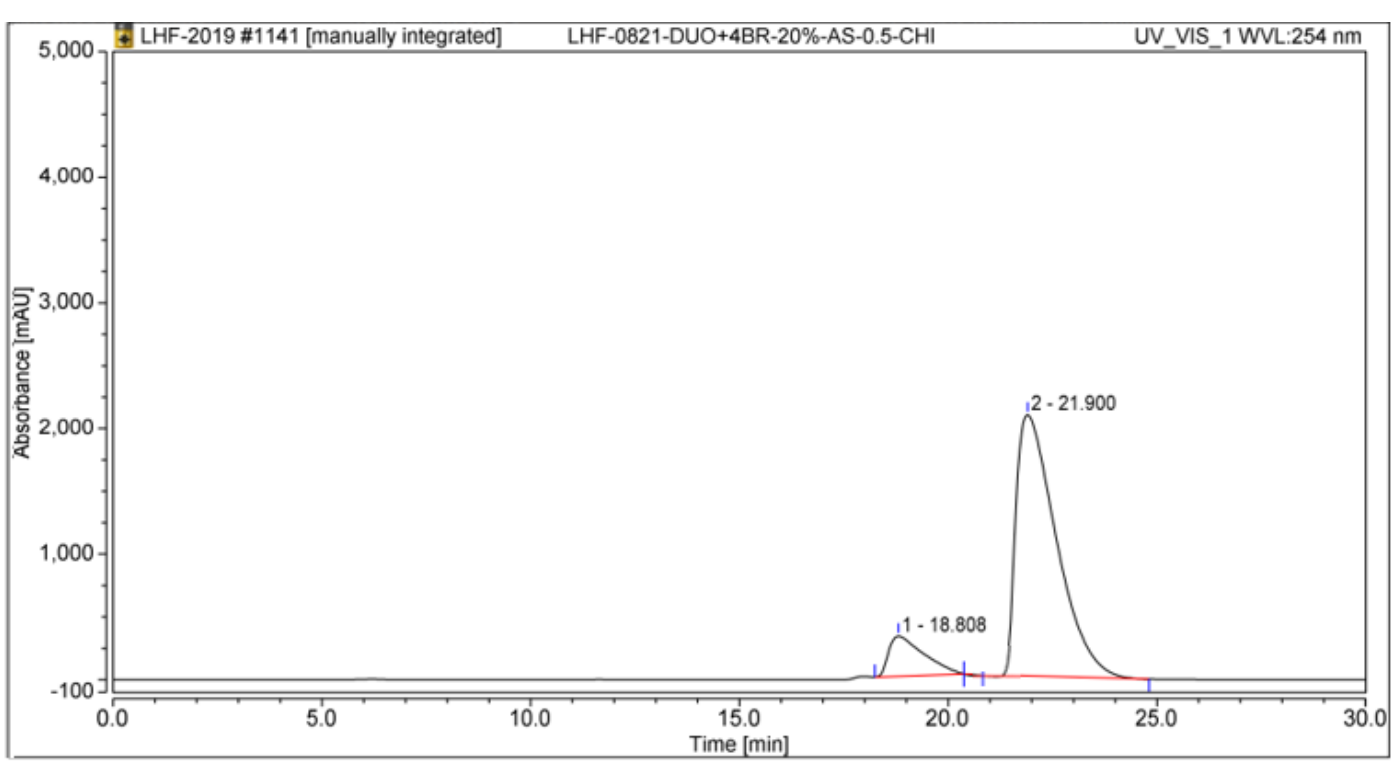

\begin{tabular}{|c|c|c|c|c|c|c|c|}
\hline \multicolumn{8}{|c|}{ Integration Results } \\
\hline No. & Peak Name & $\begin{array}{c}\text { Retention Time } \\
\text { min }\end{array}$ & $\begin{array}{c}\text { Area } \\
\text { mAU*min }\end{array}$ & $\begin{array}{l}\text { Height } \\
\text { mAU }\end{array}$ & $\begin{array}{c}\text { Relative Area } \\
\%\end{array}$ & \begin{tabular}{|c|} 
Relative Height \\
$\%$
\end{tabular} & $\begin{array}{c}\text { Amount } \\
\text { n.a. }\end{array}$ \\
\hline \begin{tabular}{|l}
1 \\
2 \\
\end{tabular} & & $\begin{array}{l}18.808 \\
21.900 \\
\end{array}$ & $\begin{array}{c}310.337 \\
2382.299 \\
\end{array}$ & $\begin{array}{r}319.673 \\
2077.679 \\
\end{array}$ & $\begin{array}{l}11.53 \\
88.47 \\
\end{array}$ & $\begin{array}{l}13.33 \\
86.67 \\
\end{array}$ & $\begin{array}{l}\text { n.a. } \\
\text { n.a. }\end{array}$ \\
\hline \multicolumn{3}{|c|}{ Total: } & 2692.637 & 2397.352 & 100.00 & 100.00 & \\
\hline
\end{tabular}



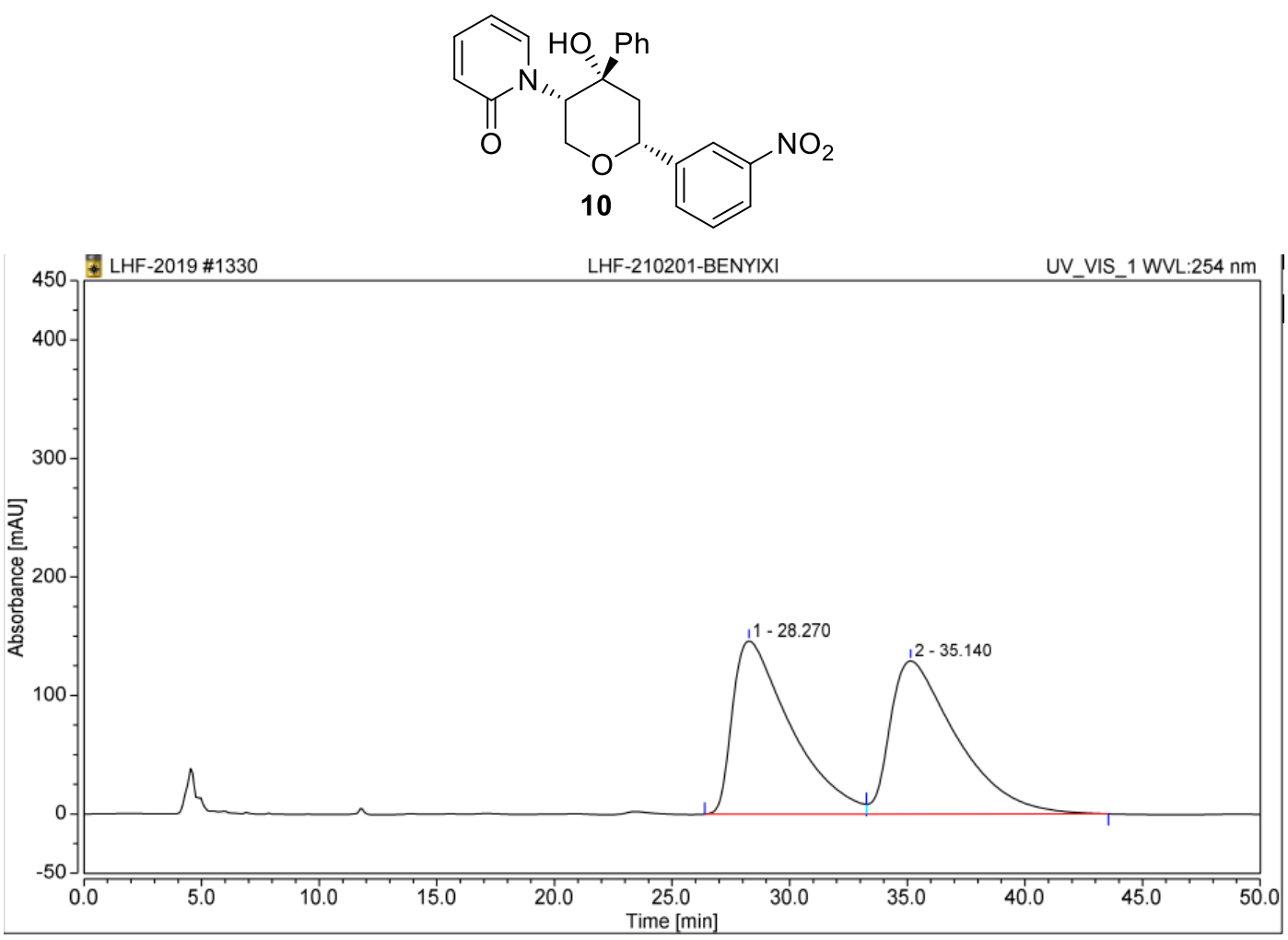

\begin{tabular}{|c|c|c|c|c|c|c|c|}
\hline \multicolumn{8}{|c|}{ Integration Results } \\
\hline No. & Peak Name & $\begin{array}{l}\text { Retention Time } \\
\text { min }\end{array}$ & $\begin{array}{c}\text { Area } \\
\mathrm{mAU}^{*} \min \end{array}$ & $\begin{array}{c}\text { Height } \\
\text { mAU }\end{array}$ & $\begin{array}{c}\text { Relative Area } \\
\%\end{array}$ & $\begin{array}{c}\text { Relative Height } \\
\%\end{array}$ & $\begin{array}{c}\text { Amount } \\
\text { n.a. }\end{array}$ \\
\hline 1 & & 28.270 & 418.517 & 145.987 & 49.76 & 53.07 & n.a. \\
\hline 2 & & 35.140 & 422.628 & 129.102 & 50.24 & 46.93 & n.a. \\
\hline \multicolumn{3}{|c|}{ Total: } & 841.145 & 275.088 & 100.00 & 100.00 & \\
\hline
\end{tabular}

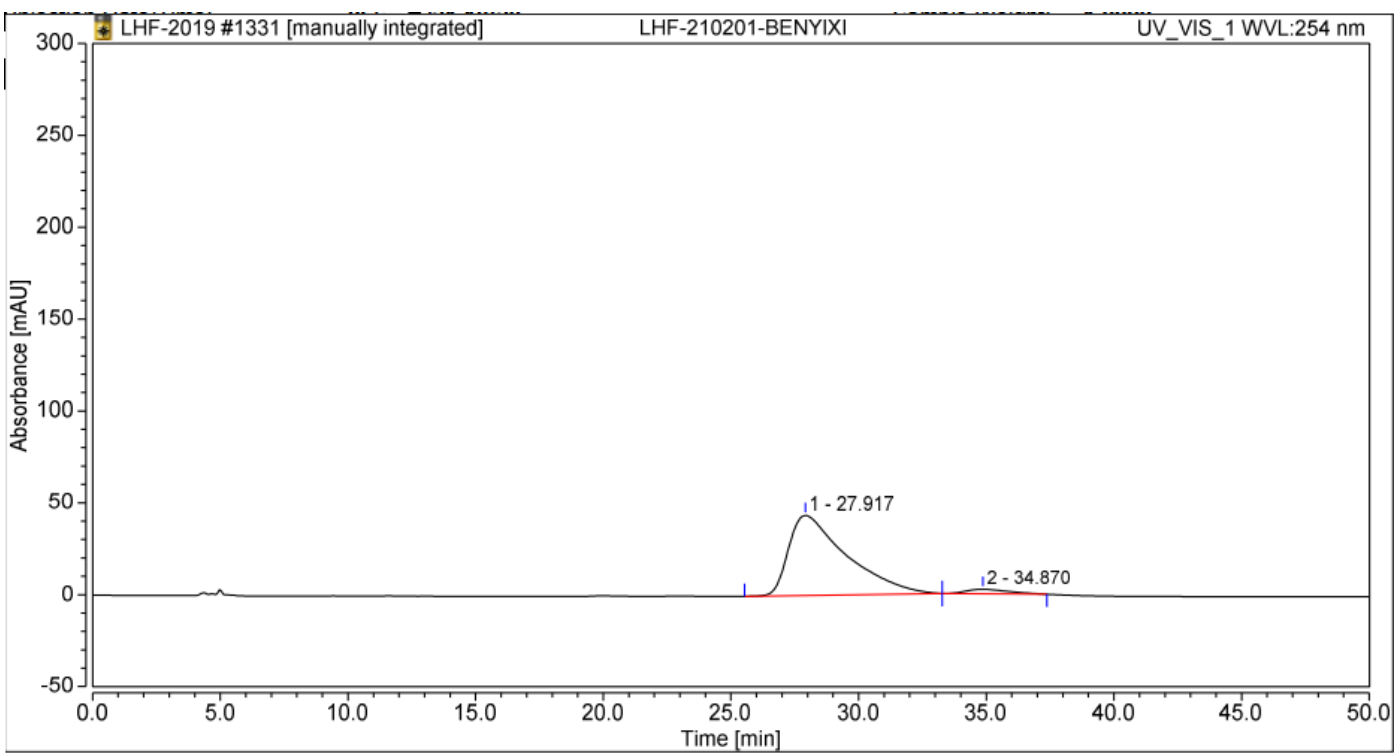

\begin{tabular}{l|l|c|c|c|c|c|c}
\hline \multicolumn{1}{|l|}{ Integration Results } \\
\hline No. & Peak Name & $\begin{array}{c}\text { Retention Time } \\
\min \end{array}$ & $\begin{array}{c}\text { Area } \\
\mathrm{mAU} \text { min }\end{array}$ & $\begin{array}{c}\text { Height } \\
\mathrm{mAU}\end{array}$ & $\begin{array}{c}\text { Relative Area } \\
\%\end{array}$ & $\begin{array}{c}\text { Relative Height } \\
\%\end{array}$ & $\begin{array}{c}\text { Amount } \\
\text { n.a. }\end{array}$ \\
\hline 1 & & 27.917 & 114.027 & 43.565 & 96.03 & 94.72 & n.a. \\
2 & 34.870 & 4.716 & 2.430 & 3.97 & 5.28 & n.a. \\
\hline
\end{tabular}

